\title{
Modeling of Outer Radiation Belt Electron Scattering due to Spatial and Spectral Properties of ULF Waves
}

\author{
Mattias Tornquist \\ West Virginia University
}

Follow this and additional works at: https://researchrepository.wvu.edu/etd

\section{Recommended Citation}

Tornquist, Mattias, "Modeling of Outer Radiation Belt Electron Scattering due to Spatial and Spectral Properties of ULF Waves" (2014). Graduate Theses, Dissertations, and Problem Reports. 289.

https://researchrepository.wvu.edu/etd/289

This Dissertation is protected by copyright and/or related rights. It has been brought to you by the The Research Repository @ WVU with permission from the rights-holder(s). You are free to use this Dissertation in any way that is permitted by the copyright and related rights legislation that applies to your use. For other uses you must obtain permission from the rights-holder(s) directly, unless additional rights are indicated by a Creative Commons license in the record and/ or on the work itself. This Dissertation has been accepted for inclusion in WVU Graduate Theses, Dissertations, and Problem Reports collection by an authorized administrator of The Research Repository @ WVU.

For more information, please contact researchrepository@mail.wvu.edu. 


\title{
Modeling of Outer Radiation Belt Electron Scattering due to Spatial and Spectral Properties of ULF Waves
}

\author{
Mattias Törnquist \\ Dissertation submitted to the College of Arts and Sciences \\ at West Virginia University \\ in partial fulfillment of the requirements for the degree of \\ Doctor of Philosophy in \\ Physics
}

\author{
Mark Koepke, Ph.D., Chair \\ Dimitris Vassiliadis, Ph.D. \\ Paul Cassak, Ph.D. \\ Duncan Lorimer, Ph.D. \\ Gary Ganser, Ph.D.
}

Department of Physics and Astronomy

Morgantown, West Virginia

2014 


\section{Abstract \\ Modeling of Outer Radiation Belt Electron Scattering due to Spatial and Spectral Properties of ULF Waves}

Mattias Törnquist

The research presented in this thesis covers wave-particle interactions for relativistic (0.5-10 $\mathrm{MeV})$ electrons in Earth's outer radiation belt $\left(r=3-7 R_{E}\right.$, or L-shells: $\left.L=3-7\right)$ interacting with magnetospheric Pc-5 (ULF) waves. This dissertation focuses on ideal models for short and long term electron energy and radial position scattering caused by interactions with ULF waves.

We use test particle simulations to investigate these wave-particle interactions with ideal wave and magnetic dipole fields. We demonstrate that the wave-particle phase can cause various patterns in phase space trajectories, i.e. local acceleration, and that for a global electron population, for all initial conditions accounted for, has a negligible net energy scattering. Working with GSM polar coordinates, the relevant wave field components are $E_{L}$, $\mathrm{E}_{\varphi}$ and $\mathrm{B}_{\mathrm{Z}}$, where we find that the maximum energy scattering is 3-10 times more effective for $\mathrm{E}_{\varphi}$ compared to $\mathrm{E}_{\mathrm{L}}$ in a magnetic dipole field with a realistic dayside compression amplitude. We also evaluate electron interactions with two coexisting waves for a set of small frequency separations and phases, where it is confirmed that multi-resonant transport is possible for 
overlapping resonances in phase space when the Chirikov criterion is met (stochasticity parameter $\mathrm{K}=1$ ). The electron energy scattering enhances with decreasing frequency separation, i.e. increasing $\mathrm{K}$, and is also dependent on the phases of the waves. The global acceleration is non-zero, can be onset in about 1 hour and last for $>4$ hours.

The adiabatic wave-particle interaction discussed up to this point can be regarded as shortterm scattering ( $\tau \sim$ hours $)$. When the physical problem extends to longer time scales $(\tau \sim$ days ) the process ceases to be adiabatic due to the introduction of stochastic element in the system and becomes a diffusive process. It has been established in Fälthammar (1965) that the local power spectral density (PSD) encountered by the particles accounts for the radial position diffusion rate $\left(\mathrm{D}_{\mathrm{LL}}\right)$, with the assumptions that the fields are stationary and ergodic. Our study does not assume ergodicity, which means that the phases of the modes can change dynamically over time. In addition to particle simulations we calculate variances in radial positions directly via integrations of the wave. The dynamic phases appear either from random resets of the modes, with a rate of $f_{r}$, or from particle exposure to waves confined in specific magnetic local time (MLT) sectors where $f_{r}$ is replaced by the drift frequency $f_{d}$ of electrons passing through.

We show that any mode in a broadband spectrum can contribute to the total diffusion rate for a particular drift frequency within the spectral band via dynamic phases. Each mode contributes maximally at a phase reset frequency $f_{r}=2.63 f_{k}$, where $f_{k}$ is the mode frequency. We experiment with electron diffusion due to interaction with wave broadband spectra in MLT sectors and find the phase reset effect being strongest when there is no azimuthal wave vector $\left(\mathrm{m}_{\mathrm{sec}}=0\right)$ within the sector. $\mathrm{D}_{\mathrm{LL}}$ rapidly coheres to the local PSD as the wave number increases and, for example, at $\mathrm{m}_{\mathrm{sec}}=1.00 \pm 0.25$ the effect of phase resets is only $10-30 \%$ as 
strong as for $\mathrm{m}_{\mathrm{sec}}=0$. Since phase resets depend on particle drift frequencies when MLT sectors are involved, a consequence is that $\mathrm{D}_{\mathrm{LL}}$ must adjust as a function of $\mathrm{L}$-shell as well. For example, from the local PSD as the sole contributor to diffusion Schulz and Lanzerotte (1979) has shown that $D_{L L} \alpha L^{6}$, but we prove that the function becomes $D_{L L} \alpha L^{5}$ with some variations due to $\mathrm{f}_{\mathrm{d}}$ and MLT sector width.

The final part of this dissertation evaluates a pre storm commencement event on November 7 , 2004, when Earth's magnetopause was struck by a high-speed solar wind with a mostly northward component of interplanetary magnetic field. We obtained a global MHD field simulated by the OpenGGC model for the interval 17:00-18:40 in universal time from NASA's Community Coordinated Modeling Center. Global distribution plots of the electric and magnetic field PSD reveal strong ULF waves spanning the whole dayside sector. There are distinct electric field modes at approximately $0.9,2.3$ and $3.7-6.3 \mathrm{mHz}$ within the dayside sector, which we then used in test-particle simulations and the variance calculations in order to evaluate the diffusion coefficients. To ensure diffusion by sufficient stochasticity, we run the event by repeating the interval 10 times in series for a total duration of 12 hours. For the wave electric fields, the predicted diffusion coefficient due to local PSD matches the outcome from simulated electron scattering at 0.9 and $2.3 \mathrm{mHz}$. The diffusion due to the wider frequency band at 3.7-6.3 $\mathrm{mHz}$ does not fit the PSD profile alone, and requires phase resets in non-resonant modes within the spectrum to yield an agreement between the calculations and the simulations. Furthermore, only $\mathrm{m}_{\mathrm{sec}}=1$ provides the correct solution. We have thus demonstrated the importance in including both the MLT sector width and wave number as additional significant factors apart from the local PSD in determining the diffusion coefficient for a realistic wave field. 


\section{Acknowledgements}

My advisor, Dimitris Vassiliadis, deserves my warmest gratitude for the enormous amount of help, guidance and support he has given me throughout this dissertation project. Without him I would possibly not have made it through to the end.

Many thanks also go to Mark Koepke, who with his expertise in laboratory plasma physics gave unique and valuable points of view on the project. Mark also hosted the offices, let me conduct experiments on his Q-machine at an early stage, and provided assistantship funds through grants from the National Science Foundation.

The remaining committee members Duncan Lorimer, Paul Cassak and Gary Ganser have also played an important part by reading my dissertation, attending my defense and given useful feedback.

I would like to acknowledge the people who have been my office mates over the years and who maintained a pleasant work environment: Michael, Paul, Sean, Bruce and Jeffrey. Especially the many discussions I have had with Jeffrey and Michael have been stimulating and helpful.

Finally, I give my thanks to my parents and sister in Sweden, for their selfless support and belief in me. It is not easy to have a close family member moving far away for many years and they too have pulled a significant load on this journey. 


\section{Contents}

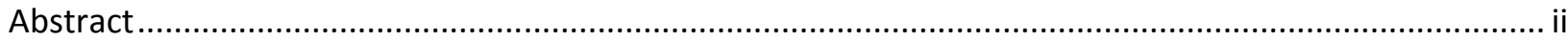

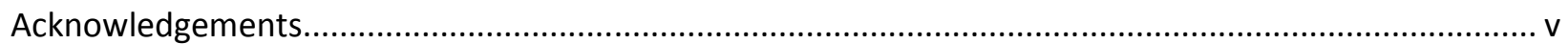

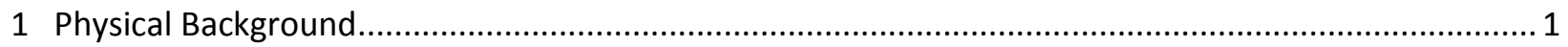

1.1 A Brief Description of the Magnetosphere and the Radiation Belts............................................... 1

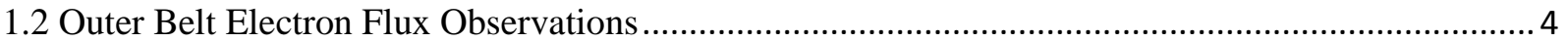

1.3 Electron Sources, Loss \& Transport …………........................................................................... 7

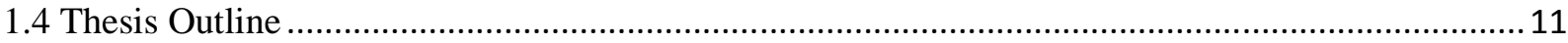

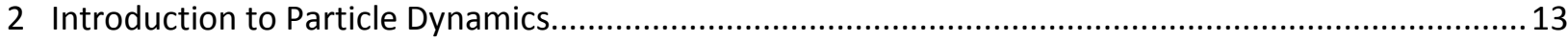

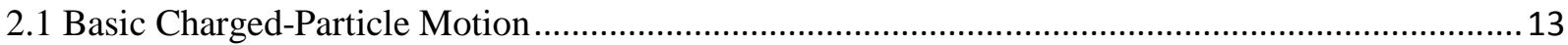

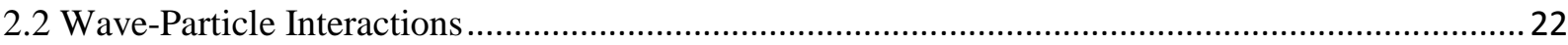

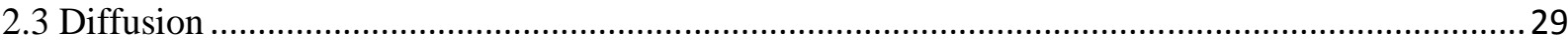

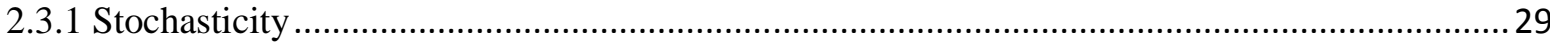

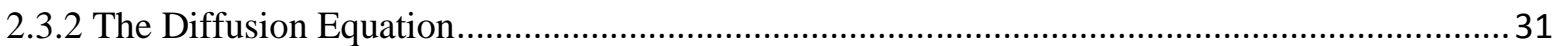

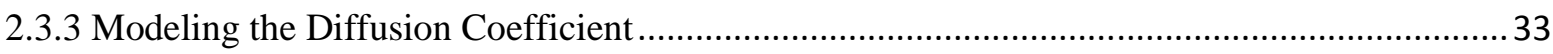

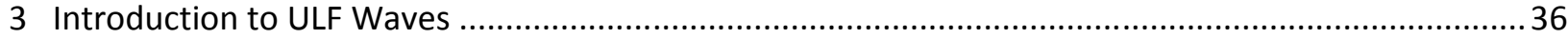

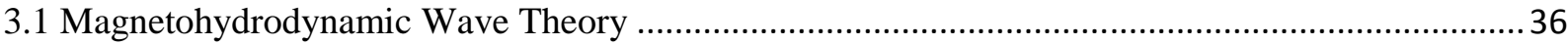

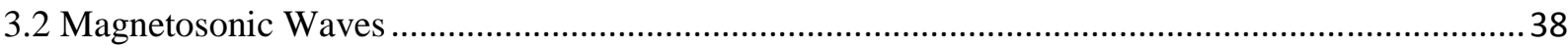

3.3 Alfvén Waves \& Field Line Resonances .................................................................................. 40

3.4 Statistical Maps of ULF Wave Occurrence and Amplitude......................................................... 44

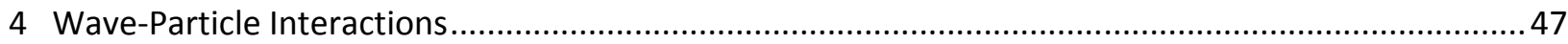

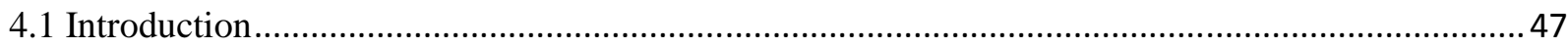

4.2 Electron Scattering by a Monochromatic Wave $\mathrm{E}_{\phi}$ Field …......................................................... 48

4.3 Electron Interactions with a Monochromatic Wave $\mathrm{E}_{\mathrm{L}}$ Field .......................................................59

4.4 Electron Interactions with a Wave Electric Field of Mixed Polarization. ......................................62

4.5 Electron Interactions with Two Coexisting $E_{\phi}$ Waves ...............................................................67

4.6 Electron Interactions with an $\mathrm{E}_{\phi}$ Wave in an MLT Sector ....................................................... 81

4.7 Electron Interactions with a Wave Electromagnetic Field......................................................... 89

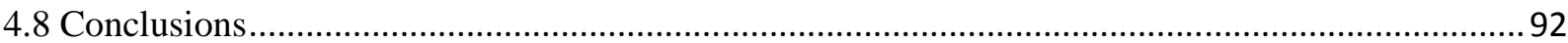

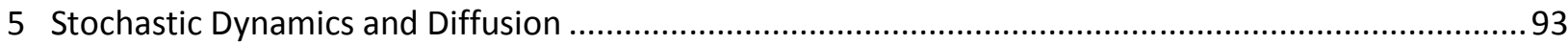

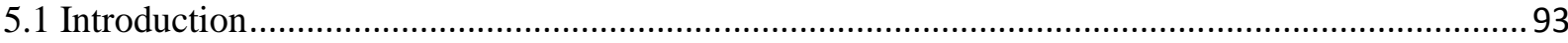




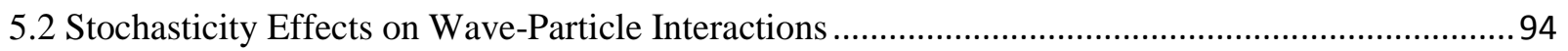

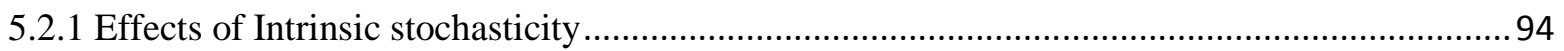

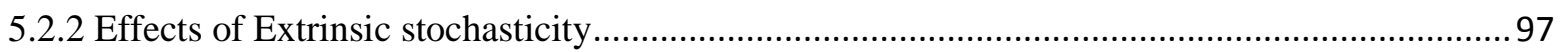

5.3 Calculating Diffusion Coefficients via the Variance Method ..................................................... 100

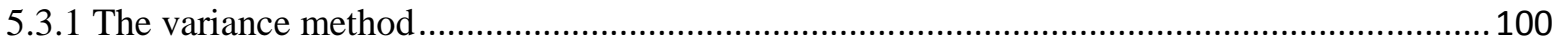

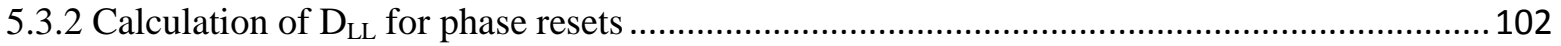

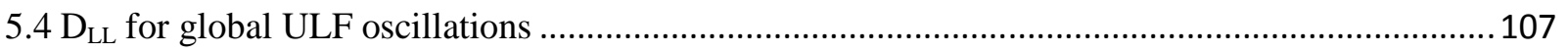

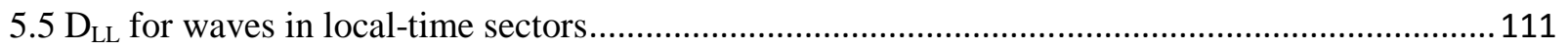

5.5.1 Demonstration with test particle simulations and variance calculations ................................. 111

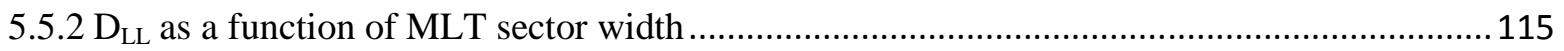

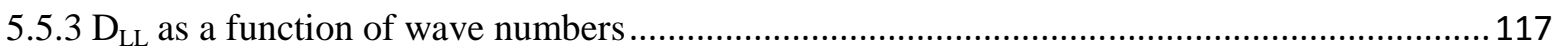

$5.6 \mathrm{D}_{\mathrm{LL}}$ as a Function of Power-law Spectral Indices .................................................................. 123

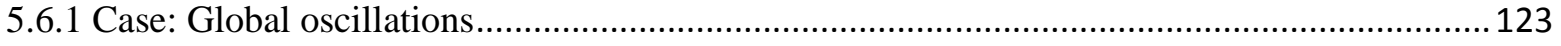

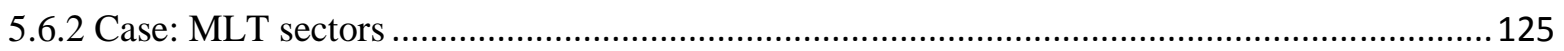

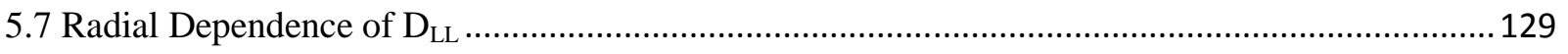

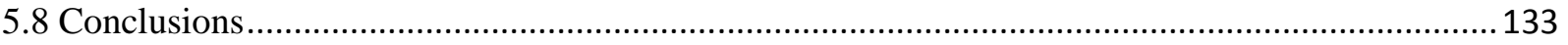

6 Event Study for Pre Storm-Commencement on November 7, 2004 ............................................. 135

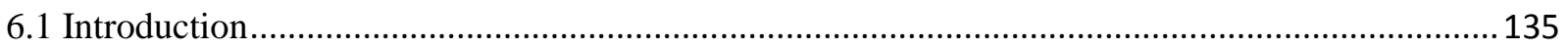

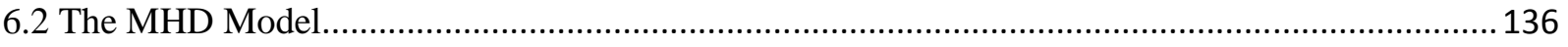

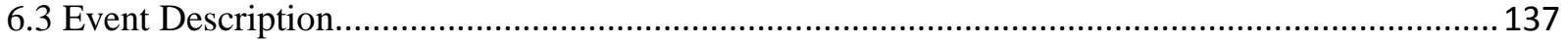

6.4 Spatial and Spectral Properties of the MHD Field........................................................................ 139

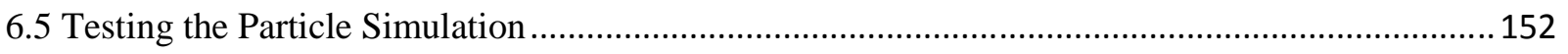

6.6 Modeling and Simulating Electron Radial Diffusion ................................................................ 157

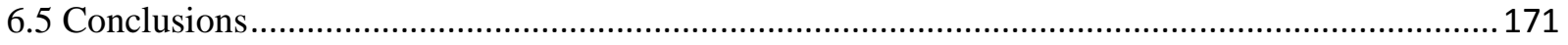

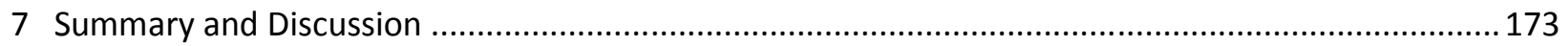

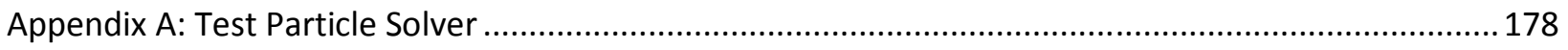

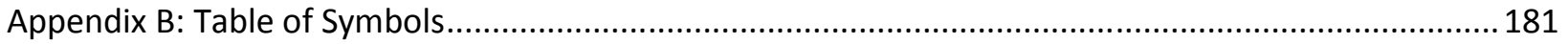

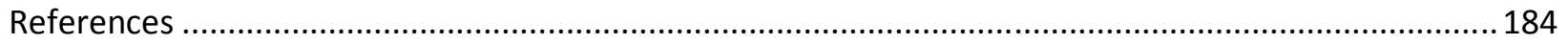




\section{Physical Background}

\subsection{A Brief Description of the Magnetosphere and the Radiation Belts}

Earth's magnetic field, produced by internal currents at Earth's core, encompasses the planet in a bubble-shaped field that resembles a dipole, and is normally referred as Earth's magnetosphere. There is constant interaction between the magnetosphere and the surrounding plasma, also known as the solar wind. The solar wind consists of ions and electrons that are emitted from the Sun and also carry frozen-in interplanetary magnetic field (IMF) with it, i.e. the field lines travel with the plasma fluid. As the dynamic pressure of the solar wind applies against the magnetosphere, the magnetosphere assumes its shape from the equilibrium boundary where the magnetic pressure counters the solar wind. This spatial boundary is known as the magnetopause [Kivelson and Russell, 1995]. For a depiction of the inner magnetosphere, see figure 1-1.

During quiet conditions, the magnetopause extends about 9-10 $R_{E}\left(R_{E}=1\right.$ Earth radius), measured from Earth's center towards the dayside on an axis between Earth and the Sun. The solar wind flow direction and velocity determines the shape of the magnetosphere. On the night side, facing away from the Sun, a tail structure with gradually compressed magnetic field lines extends in a direction along the solar wind flow on the night side. This tail is supported by an east-to-west current that spans from about $10 \mathrm{R}_{\mathrm{E}}$ out to a large distance with no distinct boundary [Walt, 1994]. 
The outer magnetosphere is thus not azimuthally symmetric and it becomes necessary to establish proper coordinates to work with. In this dissertation we will use GSM (geocentric solar magnetospheric) coordinates. GSM is defined with the origin at the center of Earth and an X-axis pointing in the direction to the Sun [Olson, 1970]. Since the approximate magnetic dipole of Earth's field is offset at about $8.7^{\circ}$ from the geographical North Pole, the z-axis is a projection of the polar direction of Earth's magnetic field onto the geocentric z-axis. The true magnetic equator is tilted from the Earth-Sun plane, but can be represented on the x-y plane in GSM coordinates with the projection. Azimuthal coordinates are appropriately represented by magnetic local time (MLT), defined as 12:00 at noon and 00:00, or 24:00, at midnight in GSM coordinates. Radial distances from Earth's center are referred to as L-shells, and defined by $L=r / R_{E}$. We will use the subscript "L" instead of "r" for all radial components.

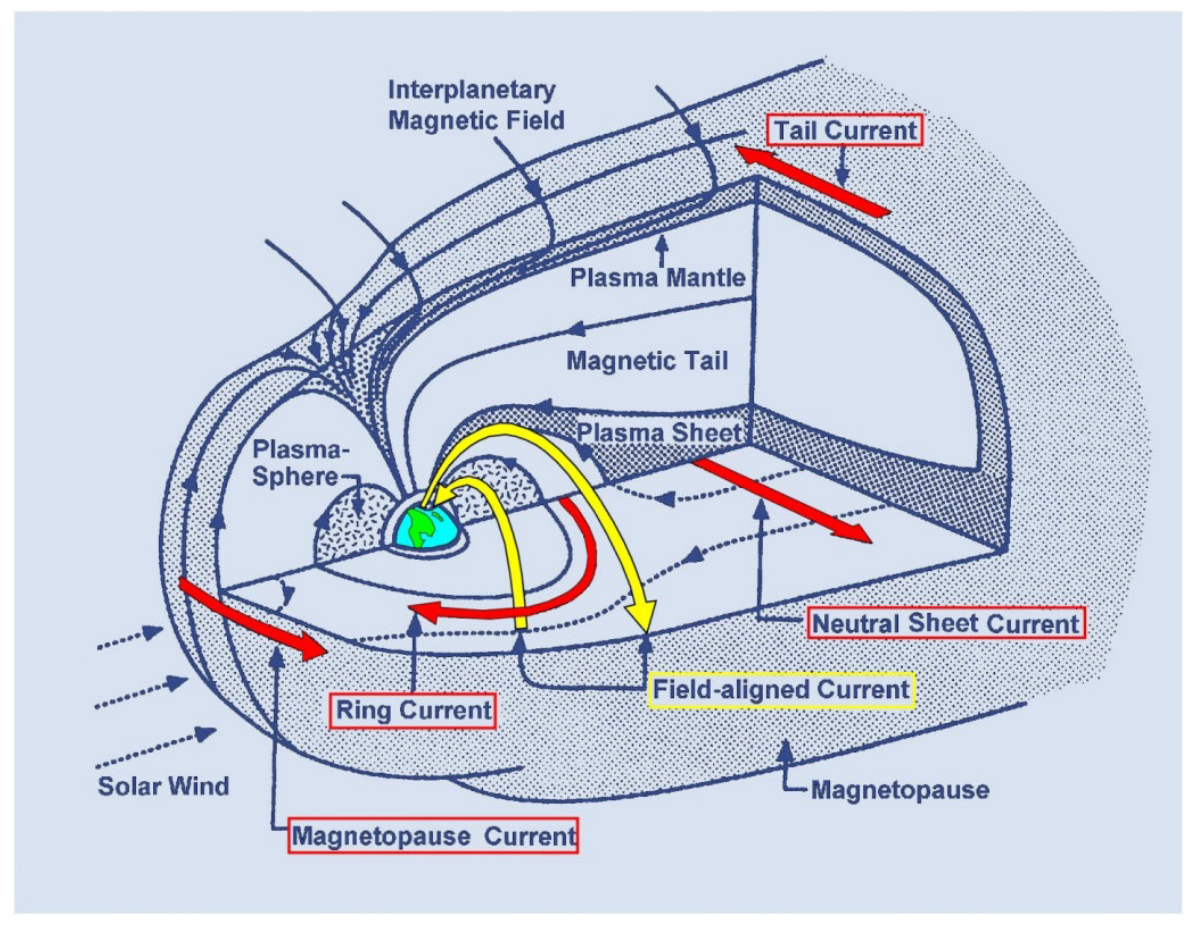

Figure 1-1: General view of the magnetosphere with its fundamental currents marked in colored arrows. [Kivelson and Russell, 1995]. 
The magnetosphere is an effective shield against solar wind particles, but as a consequence also contains trapped populations within. The regions of trapped relativistic and nearrelativistic particles are known as the radiation belts (fig. 1-2). The population of the belts consists of both ions and electrons with a wide range of energies and fluxes. Overall the belt particle fluxes have an approximately toroidal distribution, symmetric about the magnetic equator, and most of the time separated into two main belts: the inner and the outer. The inner belt extends between $\mathrm{L}=1.1-2.0$, and is rich in energetic $(>10 \mathrm{MeV})$ protons peaking at $\mathrm{L}=$ 1.5. The outer region of the radiation belt begins at about $\mathrm{L}=2.5$, where a relativistic ( 100 $\mathrm{keV}-10 \mathrm{MeV}$ ) electron population density peaks at $2.5<\mathrm{L}<5.0$. Between the two distinct belts is a region of relatively low density of energetic particles, also known as the slot region. [Walt 1994]

There are less energetic particles in the magnetosphere as well. Thermal ions and electrons comprise what is known as the ring current that spans across $3<\mathrm{L}<5$, centered at the magnetic equator, with an electron population having a typical energy range at $T_{e}=10-200$ $\mathrm{keV}$. Cold electron populations of the lowest level energies, at the order of $\mathrm{T}_{\mathrm{e}} \sim 1 \mathrm{eV}$, define the plasmasphere which has a radial extent roughly equivalent to the ring current [Goldstein, 2006].

Besides Earth, other planets such as Jupiter and Saturn can have intrinsically produced magnetospheres containing high energy particles, with their unique structure and dynamics. Wherever there are magnetic fields with large-scale fluctuations, acceleration processes are likely to develop. Astrophysical objects such as pulsars or quasars exhibit extremely energetic particle dynamics due to internal acceleration processes. Using Earth's magnetosphere as a natural laboratory can provide more insights in other natural accelerators [Walt, 1994]. 


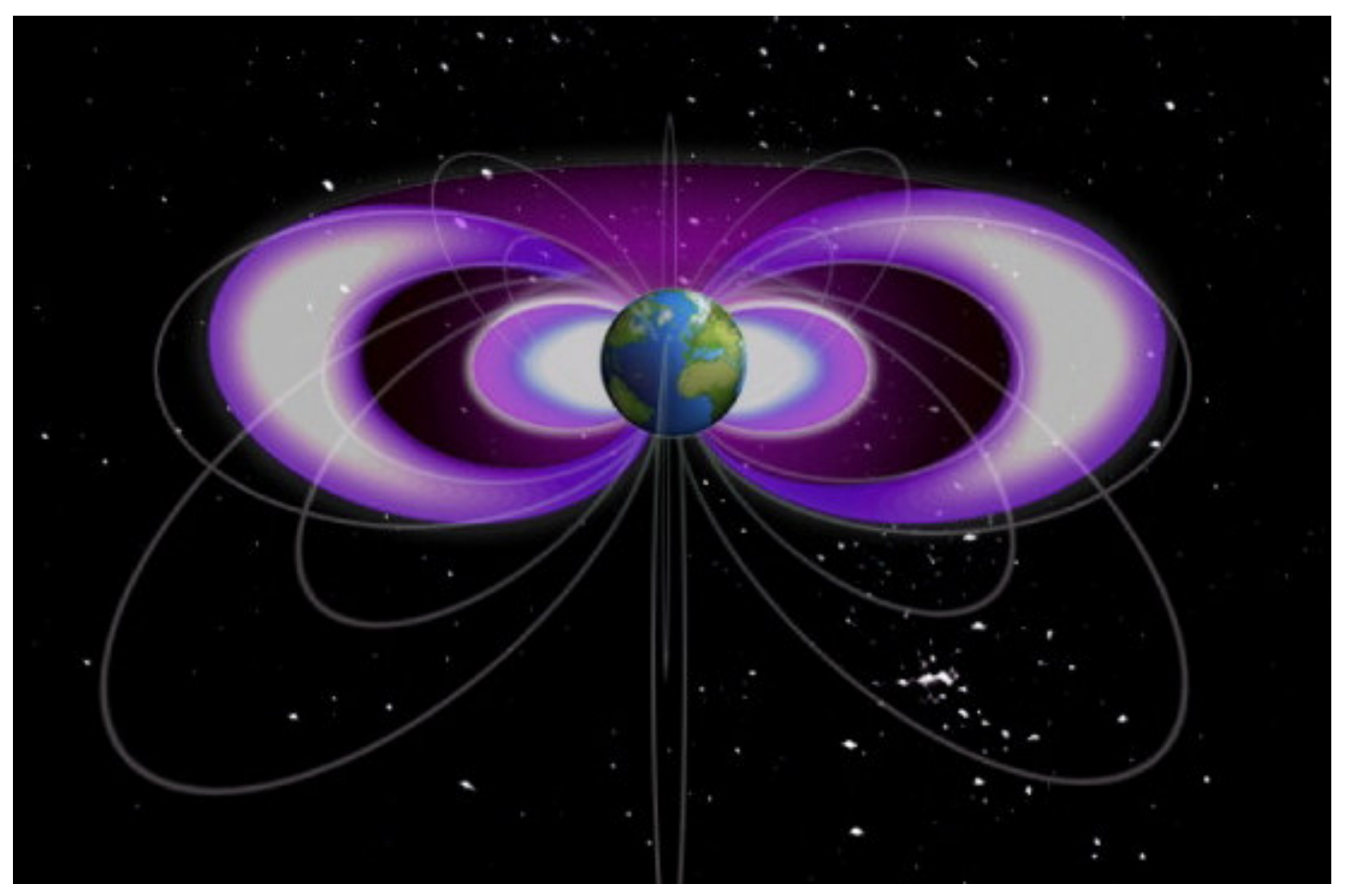

Figure 1.2: Depiction of the inner and outer radiation belts [Goldstein, 2006].

\subsection{Outer Belt Electron Flux Observations}

An early study by Williams (1966) found a relation between the ratio of kinetic energy density and magnetic energy density of the solar wind to the electron energy intensity in the outer belt. Later, Paulikas \& Blake (1979) detailed further connections between the interplanetary space environment and the outer radiation belt. They showed that enhancements of outer belt electron fluxes could be explained as functions of high solar wind speed at any time scale from a single day to half a year. In their paper a 27-day cycle trend of 
flux responses was also revealed to be consistent with the solar rotation period. This is indirectly linked to the solar wind speed. From analysis of the Combined Release and Radiation Effects Satellite (CRRES) data during the solar maximum period of July 1990 October 1991, Korth and Friedel (1996) showed that flux enhancements as well as the spatial boundaries of the outer belt were responsive to the geomagnetic activity represented by the geomagnetic indices Dst and $\mathrm{K}_{\mathrm{p}}$. The Dst (Disturbance Storm Time) index is derived from deviations in the low-latitude magnetic field horizontal component, caused by ring current enhancements. Geomagnetic storms are defined through the enhancement of the ring current and measured for large, negative values in Dst. The reason why the ring current enhances during magnetic storms is because the magnetosphere is energized by a dynamo mechanism caused by convections in the night side plasma sheet, driving currents along the field lines into the auroral zone at high latitudes, i.e. the so-called Birkeland currents. The $K_{p}$ index is a measure of the average magnetic field at Earth's surface over a global scale.

However, some studies have also shown that not all geomagnetically active periods necessarily cause enhancement of energies in the outer belt, e.g. Baker et al. (1987). Changes in the particle fluxes can vary significantly due to both timing of storm onset as well as Dst intensity as shown by Reeves (1998). In fact, Reeves (2003) showed that out of 276 geomagnetic storms studied between 1989-2000, 53\% caused increases in fluxes, 24\% showed little change, and $23 \%$ had decrease in fluxes, which suggests that the outer belt is a complex system where various mechanisms coexist (see figure 1-3). Recent observations by Baker et al. (2013) also hinted at more complex flux redistribution of electrons in the outer belt via a creation of a third radiation belt at $\mathrm{L}=3.0-3.5$ that lasted for about a month [Baker et al., 2013]. As opposed to the outer belt electrons, the ion populations of the inner belt tend 
to be far less responsive to storm events since field disturbances are relatively less effective in displacing these heavier particles. Thus with larger distance from Earth, the radiation belt is more dynamic [Walt, 1994]. There are many proposed mechanisms [Friedel et al., 2001] explaining the radiation belt dynamics; there must be sources of injection of particles, various ways to cause losses, and internal redistribution of fluxes.
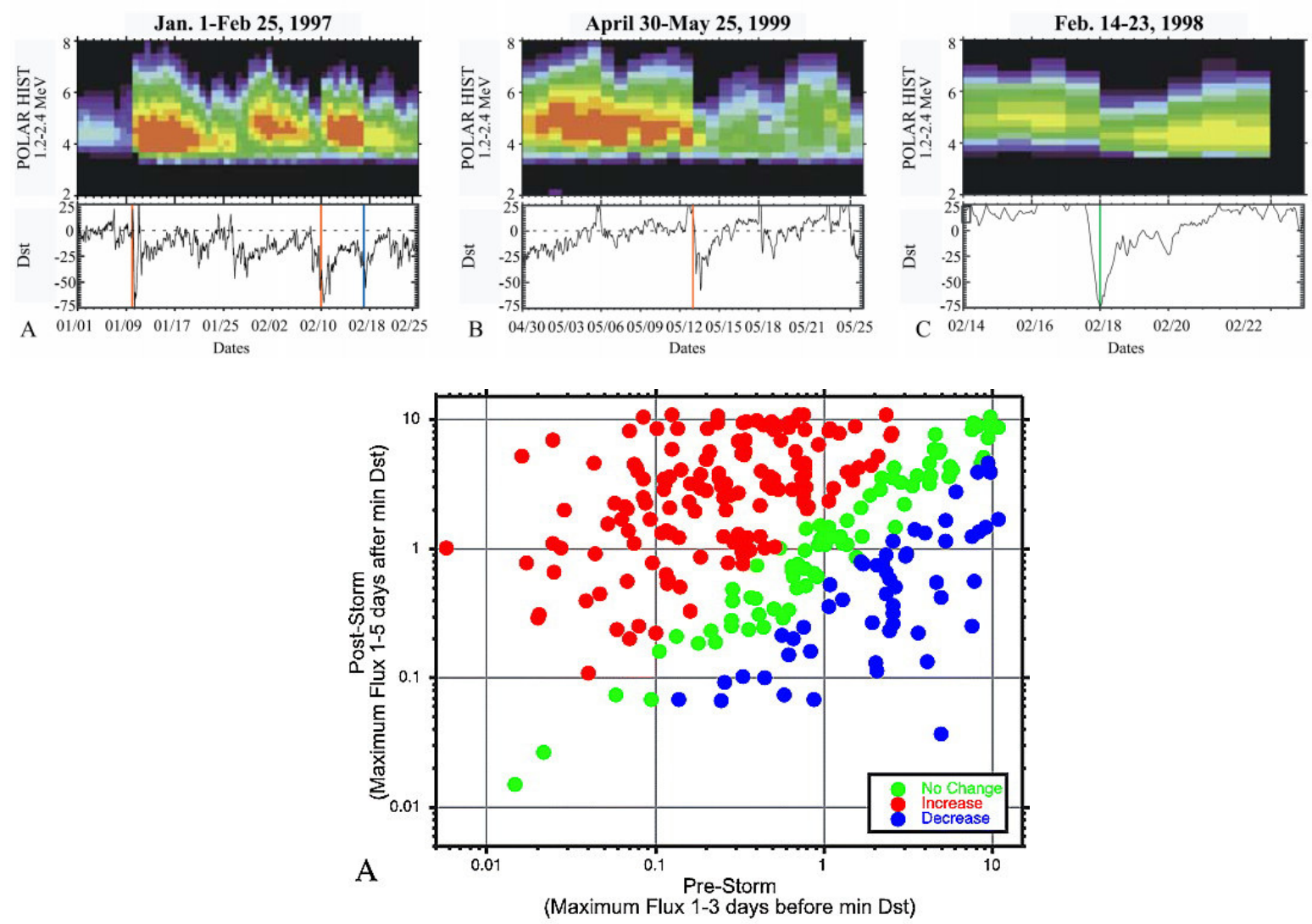

Figure 1-3: Top: Selected geomagnetic storm events where electron fluxes for energies at $W$ =1.2-2.4 MeV from POLAR satellite data is shown. Red colors show increase in flux, blue shows decrease and green shows no change. Bottom: Collected storm events sorted in electron flux change [Reeves et al., 2003]. 


\subsection{Electron Sources, Loss \& Transport}

In terms of sources, new electrons and ions can be injected into the magnetosphere in various ways. One mechanism arises in substorms when the tail of Earth's magnetosphere undergoes magnetic reconnection with the interplanetary magnetic field and rapidly forces plasma inward [Kivelson and Russell: Hughes, 1995], they can originate from the dayside cusps [e.g. Sheldon (1998)], or the ionosphere can be the source of protons, helium and oxygen ions [Walt, 1994].

Typically, particles injected in the radiation belts are not sufficiently energetic to explain the relativistic portion of the observed population and must thus require ways of enhancing their energies. One kind of dramatic events occurs for shocks, e.g. powerful coronal mass ejections colliding with Earth's magnetosphere. Sudden dayside magnetopause compressions induce strong, convective electric fields in a westward direction [Wygant et al., 1994] that transport and energize particles at the order of minutes [Kress et al., 2007]. In some rare extreme events this can lead to the creation of new belts, as for example observed for a powerful acceleration event on March 24, 1991 observed by Blake et al. (1992) - and confirmed in a simulation by Li et al. (1993). For most geomagnetic storms however, shock induced compressions are not sufficiently strong to produce the observed outer belt electron energies, thus another mechanism has been proposed: wave-particle interactions [Schulz et al., 1974]. Not only can these interactions cause changes in particle energies, but also spatially transport them (see chapter 2.2). 
There are various types of plasma waves that can be generated in the magnetosphere, i.e. whistler, ion cyclotron (EMIC), turbulent whistler (hiss) and ultra-low frequency (ULF) waves. Each type has distinct morphology, and variations in frequencies, magnitudes and durations. There is a geomagnetic pulsation classification that considers the time dependent aspect, which are divided into the classes Pc 1-5 and Pi 1-2 [Jacobs, 1964]. 'Pc' stands for continuous pulsations, being that for consistent waves lasting for time scales typically at the order of hours. The range of frequencies for all levels spans between $0.5 \mathrm{mHz}$ up to $5 \mathrm{~Hz}$, where ultra-low frequency covers Pc 4-5 (0.5-22 mHz). 'Pi' denotes irregular pulsations and the indices cover the higher frequency range of Pc-type.

\begin{tabular}{|c|c|c|c|c|c|c|}
\hline \multicolumn{5}{|c|}{ Continuous } & \multicolumn{2}{|c|}{ Irregular } \\
\hline Pc1 & Pc2 & $\mathrm{Pc} 3$ & Pc4 & Pc5 & Pi1 & $\mathrm{Pi} 2$ \\
\hline $0.2-5 \mathrm{~s}$ & $5-10 \mathrm{~s}$ & $10-45 \mathrm{~s}$ & $45-150 \mathrm{~s}$ & $150-600 \mathrm{~s}$ & $1-40 \mathrm{~s}$ & $40-150 \mathrm{~s}$ \\
\hline $0.2-5$ & $0.1-0.2 \mathrm{~Hz}$ & $22-100 \mathrm{mHz}$ & $7-22 \mathrm{mHz}$ & $2-7 \mathrm{mHz}$ & $0.025-1 \mathrm{~Hz}$ & $2-25 \mathrm{mHz}$ \\
\hline
\end{tabular}

Figure 1-4: Magnetospheric wave frequency ranges. Top row: ULF wave class. Middle row: Range of periods. Bottom row: Range of frequencies. [Jacobs, 1964]

One of the most established theories of particle loss is that of resonant scattering of particles due to whistler waves [Kennel and Petschek, 1966]. It is believed that the particle momenta become stronger along the magnetic field line direction of Earth's dipole, causing the particles to precipitate into Earth's atmosphere. This is often referred as pitch-angle scattering. It is an explanation why there is a gap of fluxes between the inner and the outer belt since there is only a finite range of particle energies and radial locations that can be resonant with these waves. 
Apart from atmospheric precipitation, outer belt particles can also become de-trapped by passing through the magnetopause, i.e. magnetopause shadowing [West et al. 1972], [Roederer et al., 1968] [Turner et al., 2012]. A related type of loss is drift-orbit bifurcation where electrons enter drift orbits at high magnetic latitudes near the cusps, effectively depleting flux in the radially central regions of the outer radiation belt [Ukhorskiy and Sitnov, 2011]. Waves could play a role in transporting particles into L-shells leading to these loss mechanisms.

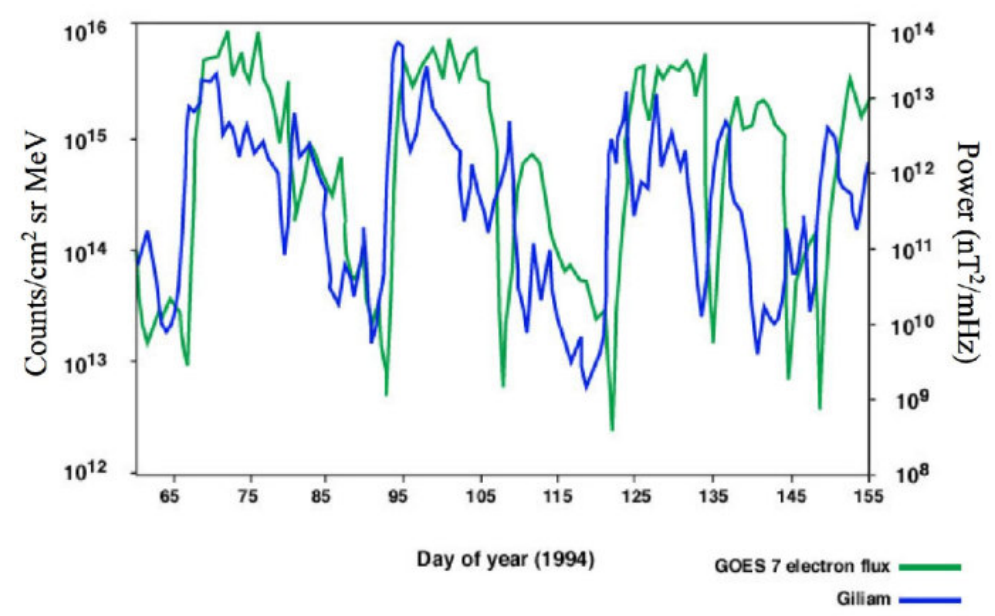

Figure 1-5: ULF wave power and outer radiation belt relativistic electron flux.

[Rostoker, 1998]

The type of wave in focus for this thesis is ULF waves, which mainly affects the outer radiation belt electron population. There are several studies linking this type of wave activity to changes in electron fluxes, from short term trends of a few hours up to a few months. Rostoker et al. (1998) showed a very clear correlation between magnetic field ULF power deduced from a ground magnetometer and particle flux measured with the GOES-7 satellite at 
geostationary orbit, as well as the SAMPEX satellite at low-Earth orbit, during 3 months in 1994 (see figure 1-5). This interval did not cover any major geomagnetic storms, but instead had intervals of high-speed downstream solar wind. As had already been suggested in Paulikas and Blake (1979), geosynchronous electron flux has a correlation with the solar wind speed. An additional step was taken by Mathie and Mann (2000) which linked the solar wind speed to ULF activity, thus indirectly another observational correlation between waves and electron fluxes can be drawn. How the solar wind drives ULF waves will be discussed in more detail in chapter 3 . There have been other various reports of ULF activity measurements during storm events and enhanced electron fluxes. For example, Mathie and Mann (2000a) showed that ULF power during geomagnetic storms can equal in strength to high-speed solar wind induced waves, and Green and Kivelson (2001) argues that geomagnetic storms in combination with large wave power are essential in transporting outer belt electrons. Connections between solar activity, ULF wave power and energy flux of relativistic electrons have been shown by for example [Mathie and Mann 2000] [O'Brien 2001] [Rostocker 1998] [Baker 1998], where the peaks of the two latter correlate over several days at Geosynchronous distance and happen as the simultaneously existing storms wane. Other studies have shown cases where no energized particles were detected during storms with enhanced ULF activity, e.g. [Baker et al., 1998] and [Reeves et al.,2003].

In light of observations described in this introduction the main challenge lies in explaining and predicting the vastly different outcomes in radiation belt electron population distribution associated with geomagnetic storm events. It is a typical feature of chaotic dynamics being involved. One main contribution to this can be ULF wave-particle interactions where initial and boundary conditions play a role. This dissertation will delve further into the electron 
scattering mechanism for short time scales of hours, which is typical for main phases of geomagnetic storms. Waves can also drive particle populations over time scales of days, which is equivalent to the recovery phase of storms. This is a diffusive process of transport and will be investigated in this dissertation as well.

\subsection{Thesis Outline}

The chapter outline is as follows:

Chapter 2: We introduce basic radiation belt dynamics learned from previous studies. Particle motions are described in an ideal, wave-free magnetosphere and during wave-particle interactions. The diffusion of electron radial distribution due to wave-particle interaction is also discussed.

Chapter 3: This chapter gives an introduction in ULF wave theory, learned from previous studies. We briefly discuss how ULF waves are generated in the magnetosphere, and what their typical spatial distribution, duration, amplitude and polarization are.

Chapter 4: The discussion in this chapter entails short-term wave-particle interactions in ideal settings. We demonstrate simulations of electron energy scattering due to interactions with monochromatic ULF waves. Cases with specific wave polarizations, 2-wave system and locally confined ULF activity are investigated.

Chapter 5: In this chapter we discuss stochastic evolution of electron energy and radial position in the outer belt. The stochasticity, which can be internally or externally imposed in a 
wave-particle system, is explained in detail. We model the diffusion rate from the wave parameters.

Chapter 6: We use a geomagnetic storm commencement event on Nov 7, 2004 as an example in evaluating the diffusion coefficients and compare the wave parameters as drivers. 


\section{Introduction to Particle Dynamics}

\subsection{Basic Charged-Particle Motion}

The motion of charged particles being exposed to electromagnetic fields ( $\mathbf{E}$ and $\mathbf{B}$ ) can be described via the Lorentz force equation:

$$
\mathbf{F}=\frac{d \mathbf{p}}{d t}=q(\mathbf{E}+\mathbf{v} \times \mathbf{B})
$$

where the momentum, $\mathbf{p}=m_{s} \mathbf{v}$, is defined for either electrons $(\mathrm{s}=\mathrm{e})$ or ions $(\mathrm{s}=\mathrm{i})$ and $\mathrm{q}$ is the particle charge (+e for protons, -e for electrons). Since only electrons are considered in this outer radiation belt study the Lorentz force can then be rewritten:

$$
\frac{d \mathbf{v}}{d t}=-\frac{e}{m_{e}}(\mathbf{E}+\mathbf{v} \times \mathbf{B})
$$

There are three basic types of motions of charged particles in the radiation belts due to the static magnetic field alone, which are: gyro motion, parallel motion with bouncing between latitudinal mirror points, and drift motion (see figure 2-1). Each of these periodic motions is associated with an adiabatic invariant that can be approximated as a constant should the motion in question be perturbed by much slower changes than the motion period [Northrop, 1963]. To distinguish these fundamental motions, the particle momentum vectors can be separated into a parallel $\left(\mathrm{p}_{\|}, \mathrm{W}_{\|}\right)$and perpendicular $\left(\mathrm{p}_{\perp}, \mathrm{W}_{\perp}\right)$ component with respect to the magnetic field lines. 


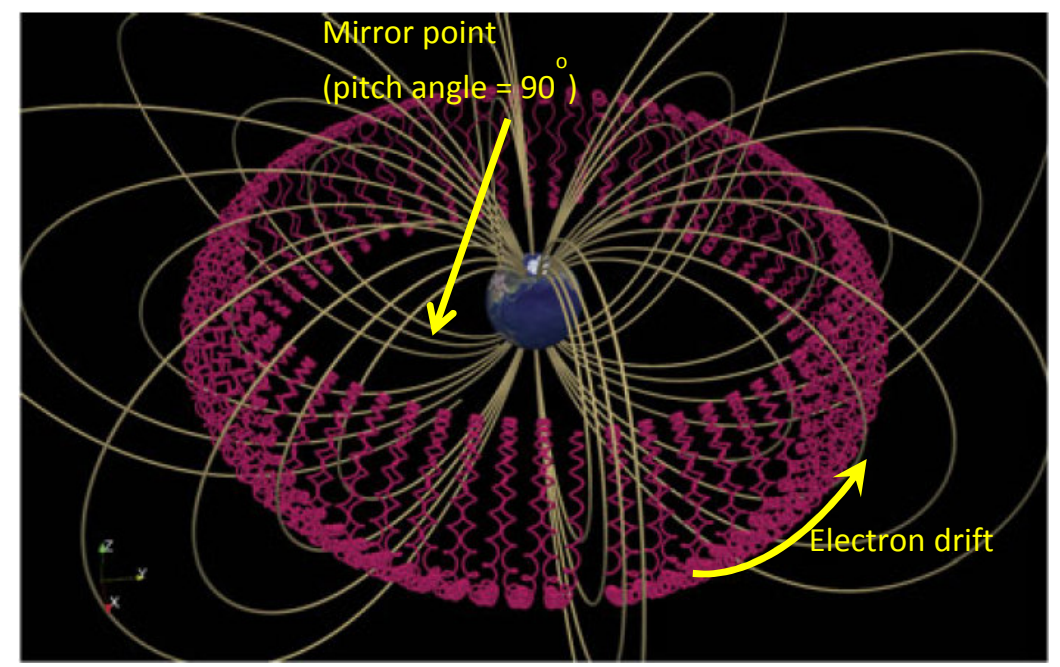

Figure 2-1: Schematic of a particle trajectory in a static dipole field.

$$
\text { [Ukhorskity and Sitnov, 2012] }
$$

The first adiabatic invariant is due to the cyclotron motion of a particle:

$$
\mu=\frac{\gamma W_{\perp}}{q\left|B_{z}\right|}
$$

where the relativistic Lorentz factor is:

$$
\gamma=\frac{1}{\sqrt{1-(v / c)^{2}}}
$$

In other words, the particle gyrates in a circle perpendicular to a magnetic field line with a gyro radius:

$$
r_{g}=\frac{p_{\perp}}{e\left|B_{z}\right|}
$$


The second adiabatic invariant preserves the particle energy throughout the bouncing motion parallel to the field:

$$
J_{2}=\oint p_{\|} d s
$$

The relation between the first and the second type is often described as the pitch angle, defined by the ratio between the momentum vector components:

$$
\alpha=\tan ^{-1}\left(\frac{p_{\perp}}{p_{\|}}\right)
$$

The third invariant allows the enclosed magnetic flux within a closed path of a particle drift motion to be constant:

$$
J_{3}=q \oint \mathbf{B} \cdot d \mathbf{S}=q \Phi
$$

The cause of this motion is the charged particle drift:

$$
\mathbf{v}_{\text {drift }}=\gamma_{m} \mu \frac{\hat{B} \times \nabla B}{B}+\frac{2 W_{\|}}{q B} \frac{\hat{R}_{c} \times \hat{B}}{R_{c}}
$$

The first term is due to gradients in the magnetic field amplitude, where the dominant component arises from the radial gradient causing an azimuthal drift. The second term is the curvature drift due to centripetal forces for a bouncing particle, where $\mathrm{R}_{\mathrm{c}}$ is the radial distance from the center of the motion curve.

An additional relativistic correction factor:

$$
\gamma_{m}=\frac{1}{\sqrt{1+2 \mu|B| / W_{\text {rest }}}}
$$


is applied to the first term of equation (2.1.8), where $\mathrm{W}_{\text {rest }}=\mathrm{m}_{\mathrm{s}} \mathrm{c}^{2}$. This correction is dependent on the magnetic field. Since any particle drift perpendicular to the magnetic contour line leads to adjustments of $\mathrm{W}_{\perp}$ according to (2.1.3) the correction term of (2.1.9) is used as an intermediate quantity with respect to any initial radial position and energy of the particle.

Due to the third invariant, in any magnetic field with a spatial gradient and no external forces, a particle strictly follows the contour lines for a constant field value. On the equatorial plane in cylindrical coordinates, $\mathrm{L}^{*}$ [Roederer 1970] is the radial distance maintaining this third invariant according to

$$
L^{*}=\frac{2 \pi \mu}{\Phi R_{E}}
$$

where the first adiabatic invariant, $\mu$, is conserved here. For a symmetric dipole $\mathrm{L}^{*}$ is constant and can be replaced by $\mathrm{L}$.
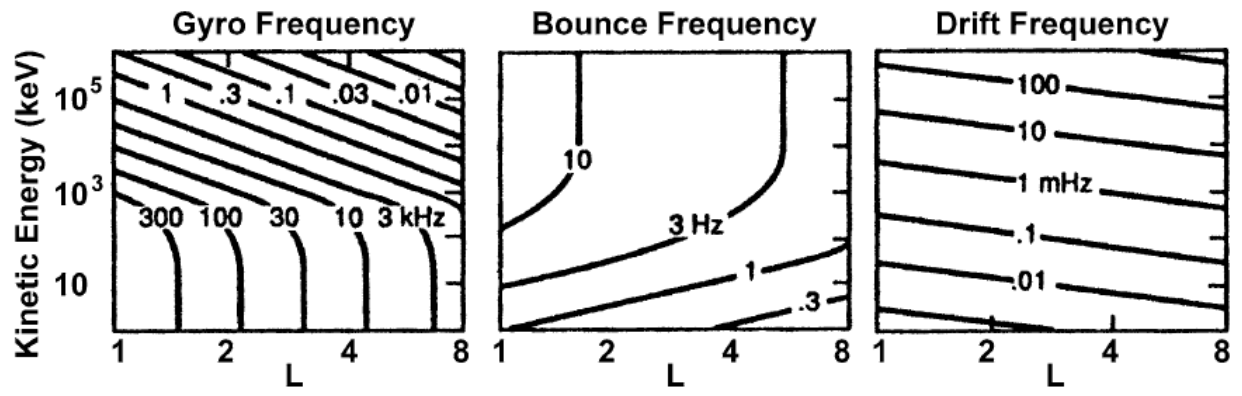

Figure 2-2: Frequency ranges for the three main types of particle motion in the radiation belts [Schulz and Lanzerotti, 1974].

All three adiabatic motion periods depend on both L-shell position and energy. Each one typically differs by many orders of magnitude from the others, as shown in figure 2-2. 
For two reasons the gyro radius can be neglected and the perpendicular momentum component be treated as a guiding-center approximation when considering bouncing and drifting motions:

1) It may be neglected due to a relatively much faster gyro period compared to the other motions when the average gyro position is approximated at the gyro center.

2) It is comparably small in proportion to the spatial dimensions of the outer belt and can be neglected in the treatment of the other motions. To give an example for a typical outer belt particle, the magnetic field at $\mathrm{L}=6$ in an ideal dipole magnetic equator is $B_{0 z}=(30500 n T) / L^{3}=140 n T$ and assuming an electron with $\mathrm{W}_{\perp}=1 \mathrm{MeV}$, the radius is $r_{\mathrm{g}}=17.0 \mathrm{~km}-\mathrm{a}$ negligible fraction of the total length scale of an electron drift path in the outer belt.

Out of these adiabatic invariants, the third one will be violated when ULF waves are introduced in the system since the frequencies are of the same order for the azimuthal drift orbits, while the other two are constant due to their shorter time scales. A consequence from the first adiabatic invariant is that a particle must adjust its perpendicular energy should it be displaced across magnetic field contours. If a particle undergoes a radially inward drift its energy, and also the azimuthal drift frequency, must increase as response. This is a fundamental part of outer radiation belt dynamics, where distributions of particles depend on radial position, drift frequency and perpendicular energy. 
This study will only consider the equatorial motions of the electrons for the reason to limit the focus solely on ULF wave interactions with electrons. Not only is it convenient to minimize degrees of freedom in a study such as this one, but there are two other reasons to make such assumption:

1) Observations implore that the ULF activity tends to be concentrated at the magnetic dipole equator, see for example Anderson et al. (1990).

2) Other observations have revealed a concentration of electron fluxes near the equator as well [Walt, 1994]. In other words their pitch angles (equation (2.1.6)) are close to perpendicular, or $90^{\circ}$. Particles with lower pitch angles have a larger likelihood in colliding with the atmosphere, thus a population of those cannot be sustained.

One should be careful about using this approximation to fully understand the dynamics of the radiation belt. There is a transition region during which equatorial electrons gradually enter increasingly parallel momenta as whistler waves begin scattering them. To theorize about this mechanism it is necessary to include both degrees of freedom for radial and parallel transport. Furthermore, Kress et al. (2007) have demonstrated the need of a 3-D model to gain a more fair understanding of radiation belt dynamics at the dayside, linked to effects such as magnetopause shadowing.

For the remainder of this section electron motion on the magnetic equatorial plane will be described in more detail. For the drift equation (2.1.8) the curvature contribution can be neglected since $\mathrm{W}_{\|}=0 \mathrm{MeV}$, but one can also add ExB-drift for the instance an electric field is introduced in the system:

$$
\mathbf{v}_{d r i f t}=\frac{\mathbf{E} \times \mathbf{B}}{B^{2}}+\gamma_{m} \mu \frac{\hat{B} \times \nabla B}{B}
$$


Separating the drift components onto cylindrical plane coordinates and using the first adiabatic invariant from equation (2.1.3) for an electron, the velocities are written as:

$$
\begin{gathered}
v_{L}=\frac{E_{\phi} B_{z}}{B^{2}}-\frac{E_{z} B_{\phi}}{B^{2}}+\frac{\gamma_{m} \mu}{B}\left(\frac{\partial B}{\partial z}-\frac{1}{L} \frac{\partial B}{\partial \phi}\right) \\
v_{\phi}=-\frac{E_{L} B_{z}}{B^{2}}+\frac{E_{z} B_{L}}{B^{2}}+\frac{\gamma_{m} \mu}{B}\left(\frac{\partial B}{\partial L}-\frac{\partial B}{\partial z}\right)
\end{gathered}
$$

where the index ' $L$ ' stands for L-shell, being the radial direction. Since $B_{L}, B_{\phi} \ll B_{z}$ and $\partial B / \partial z \ll \partial B / \partial L,(1 / L) \partial B / \partial \phi$ which terms in the drift velocities can be neglected, the drift velocities can be simplified:

$$
\begin{gathered}
v_{L}=\frac{E_{\phi}}{B_{z}}-\frac{\gamma_{m} \mu}{B_{z}} \frac{1}{L} \frac{\partial B_{z}}{\partial \phi} \\
v_{\phi}=-\frac{E_{L}}{B_{z}}+\frac{\gamma_{m} \mu}{B_{z}} \frac{\partial B_{z}}{\partial L}
\end{gathered}
$$

Assuming an azimuthally symmetric magnetic dipole field $((1 / L) \partial B / \partial \phi=0)$, azimuthal electric fields, $\mathrm{E}_{\varphi}$, cause drift in perpendicular direction to the magnetic field contour. Radially polarized fields, $\mathrm{E}_{\mathrm{L}}$, are only able to adjust the azimuthal drift velocity and without any magnetic field gradients along the azimuthal direction they cannot affect the adiabatic invariant. Thus for the case for a symmetric dipole field the azimuthal component of electric fields is the only contributor to adiabatic changes in energy via equation (2.1.3).

Considering an azimuthally symmetric dipole field, and no electric fields the azimuthal drift velocity is constant at 


$$
v_{\phi}=\frac{3 \gamma_{m} \mu}{L}
$$

where the L-shell too is constant at the initial location of a particle at $L=L_{0}$ since $v_{L}=0$.

And the azimuthal position can be given as a function of the constant drift frequency $\omega_{\mathrm{d}}$ :

$$
\phi=\omega_{d} t+\phi_{0}
$$

However if the magnetic dipole is azimuthally asymmetric particles have both radial and azimuthal drift velocities. For an asymmetric dipole field an ideal sinusoidal term can be added such that:

$$
B_{z}=\frac{B_{z 0}}{L^{3}}+B_{c} \cos \phi=\frac{B_{z 0}}{L^{3}}+B_{c 0}-B_{c 1} L \cos \phi
$$

where $B_{z 0}$ is Earth's magnetic dipole field at the surface $\left(1 R_{E}\right) \cdot \varphi=0$ is defined locally as noon with a clockwise rotation looking from north, which results in maximum compression facing noon, i.e. it is a day-night asymmetry. The L-dependence in the third term creates a more realistic magnetic field model as the magnetospheric field becomes more dipolar with decreasing L-shells. If no electric fields are assumed and this magnetic field is used in equations (2.1.14) and (2.1.15), the drift velocities become:

$$
\begin{gathered}
v_{L}=-\frac{\gamma_{m} \mu}{B_{z}} \frac{1}{L} \frac{\partial}{\partial \phi}\left[\frac{B_{z 0}}{L^{3}}+B_{c} \cos \phi\right]=\frac{\gamma_{m} \mu}{B_{z}} B_{c 1} \sin \phi \\
v_{\phi}=\frac{\gamma_{m} \mu}{B_{z}} \frac{\partial}{\partial L}\left[\frac{B_{z 0}}{L^{3}}+B_{c} \cos \phi\right]=\frac{\gamma_{m} \mu}{B_{z}}\left[-\frac{3 B_{z 0}}{L^{4}}-B_{c 1} \cos \phi\right]
\end{gathered}
$$


This pair of differential equations has to be solved numerically. The solution dictates a dependence on MLT for both velocity components. Therefore the particles spend more time at the dayside in addition to drifting further out from Earth. [Roederer 1970]

Now assuming a compressed magnetic dipole field and omitting an L-shell dependence of the compression term (2.1.18):

$$
B(L, \phi)=\frac{B_{0}}{L^{3}}+B_{c 0}-B_{c 1} \cos \phi
$$

the path of an adiabatic particle can be derived as [Elkington et al., 2003]:

$$
L(\phi)=\ell\left(1-\frac{B_{c 0} B_{c 1}}{B_{z 0}} \ell^{3} \cos \phi\right)^{-1 / 3}
$$

where

$$
\ell=\left(\frac{1}{L_{i}^{3}}+\frac{B_{c 0} B_{c 1}}{B_{z 0}} \cos \phi_{0}\right)^{-1 / 3}
$$

The parameter $\ell$ is closely related to $\mathrm{L}^{*}$ with the difference of being the averaged L-shell conserving the magnetic flux throughout an orbit - aligning with the particle location at the dawn and dusk points.

From Taylor expansions in L of equations (2.1.22) and (2.1.23) the radial difference between the extreme points of the trajectory can be summarized as:

$$
\delta L=\frac{1}{2}\left(L_{\text {noon }}-L_{\text {midnight }}\right) \simeq \frac{B_{c 0} B_{c 1}}{3 B_{z 0}} \ell^{4}
$$

The radial position of a particle can then be written as: 


$$
L(\phi) \simeq \ell+\delta L \cos \phi
$$

An instantaneous radial drift velocity is:

$$
v_{L}(\phi)=-\dot{\phi} \delta L \sin \phi
$$

which is of the same form as equation (2.1.19).

The azimuthal drift can be given a similar treatment, ending up as:

$$
v_{\phi}(\phi) \simeq \bar{v}-\delta v \cos \phi
$$

where the constant coefficients are:

$$
\begin{gathered}
\bar{v}=\frac{3 \gamma_{m} \mu}{\ell\left(1+\left(B_{c, 0} / B_{0}\right) \ell^{3}\right)} \\
\delta v=4 \gamma_{m} \mu \frac{\left(B_{c, 0} B_{c, 1} / B_{0}\right) \ell^{3}}{\ell\left(1+\left(B_{c, 0} / B_{0}\right) \ell^{3}\right)}
\end{gathered}
$$

These velocity components are going to be used in the following section for the wave-particle problem.

\subsection{Wave-Particle Interactions}

ULF waves in the inner magnetosphere are electromagnetic, thus can have significant amplitudes in both electric and magnetic fields. However, the energy density of relativistic electrons in the outer radiation belt is much smaller than the typical ULF wave energy, thus 
the wave-particle interaction taking place is approximated as a non-damping process [Dungey 1965]. Also, the wave periods are of the orders of minutes and proportional to the $3^{\text {rd }}$ adiabatic invariant in the outer electron belt, which makes it possible to consider the $1^{\text {st }}$ and sometimes the $2^{\text {nd }}$ adiabatic invariant as conserved.

Elkington et al. [1999, 2003] investigated monochromatic (discrete frequency) wave electric fields interacting with outer belt electrons and reached the following results given in this section. A simple form of a propagating mode was assumed on the magnetic equator:

$$
\mathbf{E}(\phi, t)=\mathbf{E}_{\mathbf{0}} \sin (m \phi-\omega t+\varphi)
$$

Electron drifts on the magnetic equator can be driven by the azimuthal component, $\mathbf{E}_{\mathbf{0}}=E_{0 \phi} \hat{\phi}$ , and the radial component, $\mathbf{E}_{\mathbf{0}}=E_{0 L} \hat{L}$.

The spatial form of the wave function is the relative position of the particle with respect to the wave nodes, thus can be translated as:

$$
\phi=\omega_{d} t
$$

where $\omega_{d}$ is the drift orbit frequency. The wave is assumed to span all magnetic local times, i.e. it is global, and the azimuthal wavenumber is defined accordingly with no end points.

The rate of change in energy for an interacting particle is given by [Northrop, 1963]:

$$
\frac{d W}{d t}=q \mathbf{E} \cdot \mathbf{v}_{\mathbf{d}}+\gamma_{m} \mu \frac{\partial B}{\partial t}
$$


where $q=-e$. For the even wavenumber model described in chapter 3-2 one can assume $\partial B / \partial t=0$ at the magnetic equator. For the case of using a toroidal mode wave with the polarization $\mathbf{E}_{\mathbf{0}}=E_{0 \phi} \hat{\phi}$, the resonant conditions are derived as follows:

When (2.1.15) is used for the integration of equation (2.2.3), also assuming an L-shell displacement due to the wave scattering effect being relatively small to the initial position: $\Delta L \ll L_{i}$, the outcome is [Elkington et al., 2003]:

$$
\begin{aligned}
& W-W_{0}=-e \int E_{\phi} v_{\phi} d t=-e E_{\phi 0} \frac{3 \gamma_{m} \mu}{L_{i}} \int \sin (m \phi-\omega t+\varphi) d t= \\
& =-e E_{\phi 0} \frac{3 \gamma_{m} \mu}{L_{i}} \frac{\cos \left(\left(m \omega_{d}-\omega\right) t+\varphi\right)}{\left(m \omega_{d}-\omega\right)}
\end{aligned}
$$

where the resonant condition is

$$
m \omega_{d}=\omega
$$

For an asymmetric dipole the integration becomes [Elkington et al., 2003]:

$$
\begin{aligned}
& W-W_{0}=-e \int E_{\phi} v_{\phi} d t=-e E_{\phi 0} \int \sin (m \phi-\omega t+\varphi)(\bar{v}-\delta v \cos \phi) d t \\
& =-e E_{\phi 0} \int\{\bar{v} \sin (m \phi-\omega t+\varphi)+\delta v[\sin (\omega t+(m+1) \phi-\varphi)+\sin (\omega t+(m-1) \phi-\varphi)]\} d t
\end{aligned}
$$

An assumption of a constant, averaged azimuthal drift velocity is made as per equation (2.1.17) so that the integration is solvable to:

$$
\begin{aligned}
& W-W_{0}= \\
& e E_{\phi 0}\left\{\bar{v} \frac{\cos \left(\left(m \omega_{d}-\omega\right) t+\varphi\right)}{\left(m \omega_{d}-\omega\right)}+\delta v\left[\frac{\cos \left(\left(\omega+(m+1) \omega_{d}\right) t-\varphi\right)}{\left(\omega+(m+1) \omega_{d}\right)}+\frac{\cos \left(\left(\omega+(m-1) \omega_{d}\right) t-\varphi\right)}{\left(\omega+(m-1) \omega_{d}\right)}\right]\right\}
\end{aligned}
$$


Here the resonant conditions are not only for equation (2.2.5) but also:

$$
(m \pm 1) \omega_{d}=\omega
$$

If the same procedure is followed for the wave polarization of $\mathbf{E}_{\mathbf{0}}=E_{0 L} \hat{L}$, there must be an asymmetric term in the dipole field for any energization to occur. If it assumes the same form as for the previous case, the resonant condition is equation (2.2.8) only.

A guiding-center drift simulation evaluated the location for particles, as well as their energies, over time, in order to understand the resonant wave-particle interactions. They populated an ideal magnetic dipole of the form (2.1.21) with electrons, and let the particles interact with a monochromatic $E_{L}$ wave of the form (2.2.1). The result is shown in figure 3.3 in the form of a Poincare surface of section, i.e. the phase space for the electrons is sampled at the rate of $f_{\text {sampling }}=f_{w}$. This enables separatrices to become associated with the wave cycle become visible in phase space.

A distinct resonance appears at an electron energy of $\mathrm{W}_{0} \approx 3.2 \mathrm{MeV}$ with either open or closed phase space curves that each electron creates depending on their energy (drift frequency). The phase space trajectory here is reversible and undergoes an approximate cycle with the period:

$$
\tau_{w-d}=\frac{2 \pi}{\Omega_{w-d}}=\frac{2 \pi}{\omega_{w}-m \omega_{d}}
$$




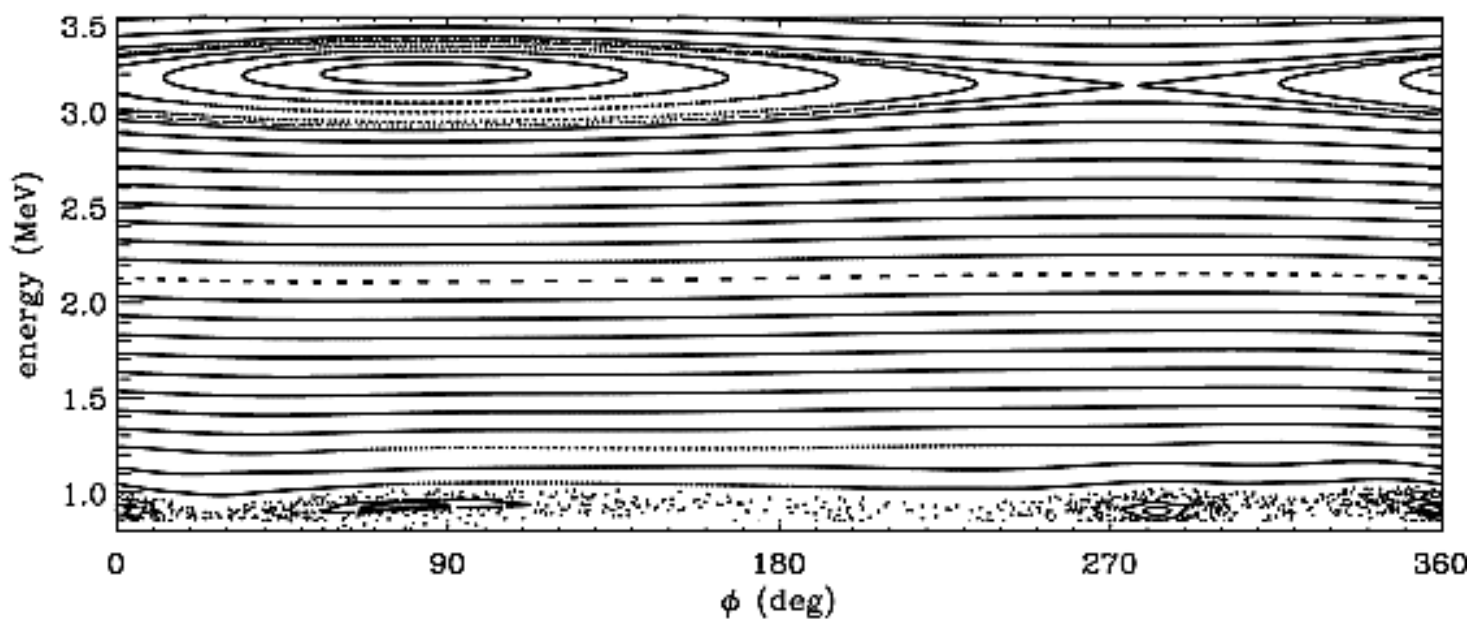

Figure 2-3 Poincare surface of section in phase space for electrons interaction with a monochromatic wave $E_{L}$ field. The background magnetic dipole field has a day-night asymmetry. [Elkington et al., 1999].

This simulation only showed a local population of electrons interacting with a monochromatic wave at a specific phase. More detailed studies in this topic, such as evaluations for other phases as well as an expansion into two waves, will be discussed in chapter 4 .

An item of interest is the resonance width in phase space. It is possible to approximate it analytically. Starting from equation (2.2.3), Degeling et al. [2007] obtained the following relation for the resonance width in L-shell scattering caused by a wave electric field with an azimuthal component interacting with electrons in a symmetric dipole:

$$
\Delta L_{\max }=2 L_{0}^{2} \sqrt{\frac{E_{0}}{\omega R_{E} B_{z 0}\left(1+3 / \gamma_{m}^{2}\left(L_{0}\right)\right)}}
$$

This is the maximal extent a particle can be transported by a single wave. 
Through straightforward binomial approximations to the first order it is possible to describe linear shifts in energy and drift frequency for particles undergoing resonant interaction with a single wave. Starting with the frequency, the shift is:

$$
\begin{aligned}
& \Delta f_{d, \max }=f_{d, f}-f_{d, 0}=\frac{3 \mu}{2 \pi}\left(\frac{\gamma_{m, f}}{L_{f}^{2}}-\frac{\gamma_{m, 0}}{L_{0}^{2}}\right)=\frac{3 \mu}{2 \pi}\left(\frac{\gamma_{m, f}}{\left[L_{0}+\Delta L_{\max }\right]^{2}}-\frac{\gamma_{m, 0}}{L_{0}^{2}}\right) \\
& \approx \frac{3 \mu}{2 \pi L_{0}^{2}}\left(\gamma_{m, f}-\gamma_{m, 0}-\frac{2 \gamma_{m, f} \Delta L_{\max }}{L_{0}}\right)
\end{aligned}
$$

where index ' 0 ' stands for initial, and ' $\mathrm{f}$ ' for final. The relativistic correction term $\gamma_{\mathrm{m}, \mathrm{f}}$ at the final location also contains $\Delta \mathrm{L}$, but for simplicity $\mathrm{L}$ remains constant in this term. Similarly for the energy:

$$
\begin{aligned}
& \Delta W_{\max }=W_{f}-W_{0}=\mu\left(\gamma_{m, f}\left|B_{f}\right|-\gamma_{m, 0}\left|B_{0}\right|\right)=\mu B_{0}\left(\frac{\gamma_{m, f}}{L_{f}^{3}}-\frac{\gamma_{m, 0}}{L_{0}^{3}}\right)= \\
& \approx \frac{\mu B_{0}}{L_{0}^{3}}\left(\gamma_{m, f}-\gamma_{m, 0}-\frac{3 \gamma_{m, f} \Delta L_{\max }}{L_{0}}\right)
\end{aligned}
$$

Since the azimuthal drift velocity is dependent on the radial location there is an asymmetry in the phase space extremes. In effect, these analytical approximations of maximal phase space widths include the assumption that $\max (\Delta \mathrm{L})=\left(\max \left(\Delta \mathrm{L}_{+}\right)+\max \left(\Delta \mathrm{L}_{-}\right)\right) / 2$. A particle slows down when drifting radially outward, thus becomes exposed to the wave throughout longer intervals as it approaches the turning point in phase space compared to a particle which is at the other side of the separatrix and speeds up instead. The consequence is a larger maximal shift in phase space $\left(\Delta \mathrm{L}_{+}, \Delta \mathrm{W}_{\text {- or }} \Delta \mathrm{f}_{\mathrm{d},-}\right)$ for particles drifting outward, compared to the inward drift particles $\left(\Delta \mathrm{L}_{-}, \Delta \mathrm{W}_{+}\right.$or $\left.\Delta \mathrm{f}_{+}\right)$. For example, an electron launched at $\mathrm{L}_{\mathrm{i}}=6.0$ and 
transported $\Delta \mathrm{L}= \pm 0.1$ would have a proportionality in drift frequency change to $\left|\frac{\Delta_{+} f_{d}}{\Delta_{-} f_{d}}\right| \propto\left|\frac{1 / L_{f+}^{2}-1 / L_{i}^{2}}{1 / L_{f-}^{2}-1 / L_{i}^{2}}\right|=\left|\frac{1 / 6.1_{f+}^{2}-1 / 6.0^{2}}{1 / 5.9_{f-}^{2}-1 / 6.0^{2}}\right|=0.967$

Elkington et al. [1999] derived a similar function for the case of a wave $\mathrm{E}_{\mathrm{L}}$ field interacting with electrons in an asymmetric dipole:

$$
\Delta W_{m \pm 1}=\sqrt{\frac{2 e E_{L 0} \delta L}{(m \pm 1)\left[\frac{\partial \ln \left(\omega_{d}\right)}{\partial W}\right]_{W=W_{m \pm 1}}}}
$$

This too has a square root relation with the wave amplitude, and also the magnetic compression which is directly found by $B_{c} \approx 3 B_{0} \delta L / L_{0}^{4}$. The wave $\mathrm{E}_{\mathrm{L}}$ amplitude can, together with the magnetic compression, contribute in scattering electrons in the outer belt. The resonance width will also be an important factor to be considered in chapter 4 .

Systems with two or more simultaneous waves are also a possibility, where particle transport across both resonances can occur should the waves be sufficiently close in frequency. Chirikov's criterion is a well-known measure that defines the onset of multi-resonance transport. It is best visualized by phase space trajectories caused by one wave that must intersect with a resonance of another wave in order to able transportation of particles across both resonances. The overlap parameter is fundamentally given by:

$$
K=\left(\frac{\Delta f_{d, \max }}{\Delta f_{k, k-1}}\right)^{2}
$$

, where $\Delta \mathrm{f}_{\mathrm{k}, \mathrm{k}-1}$ is the frequency separation between the two waves the particles interact with. 
According to Chirikov, if $K \geq 1$ then the system allows multi-resonance scattering. Not only will particle interaction with monochromatic waves be discussed in chapter 4 , but also for 2wave systems where the choice of phases, as well as the frequency separation, will turn out to be significant factors.

It is also the threshold to chaotic motion, where electron trajectories become more sensitive to initial conditions [Liebermann, chapter 4.1b] [Chirikov, 1960]. Due to the chaotic element, particle interaction with a system with multiple waves does automatically lead to diffusive scattering for sufficiently long time scales, which will be described in more detail in chapters 2.3 and 5 .

\subsection{Diffusion}

\subsubsection{Stochasticity}

If charged particles are exposed to randomly fluctuating, or sometimes described as stochastic, electromagnetic fields their trajectories would also follow random paths. This type of motion is better known as diffusion, and has historically been described as collisional dynamics. For example, in an ideal, neutral gas a particle has a deterministic path before it collides with another particle, after which both particles are placed onto different paths. Since many particles undergo these collisions, the phase-space distribution changes, which is the definition of diffusion. In the physics of the outer radiation belt electrons do not collide with each other, but instead as an analogue decorrelate in phases with the wave or waves that they 
interact with. It can appear in a system for any number of waves, even for the monochromatic case, as long as proper conditions are in place, which will be described next.

The general approach to stochastic dynamics is to introduce a number of waves surpassing the two-wave system as discussed in section 2.2, i.e. assuming a broadband spectrum. When multiple waves overlap so that $K \geq 1$ for many adjacent modes at the local frequencies, the particle motions become chaotic with high sensitivity to all phases involved. The decorrelation between particles and the wave phases introduces randomization into a system. To better illustrate this, a two-wave system is assumed to interact with a resonant particle where the initial conditions are well defined. The waves have different frequencies, thus the phase difference between them is time dependent. After a time span of:

$$
\tau_{\Delta f}=\frac{1}{\Delta f_{k, k-1}}
$$

the phase between the waves has become equal to its initial value - they are back in their starting configuration. The particle however is not at its initial position when this occurs since the cycle frequency found in equation (2.2.9) is $1 / \Omega_{\mathrm{NL}} \neq 1 / \Delta \mathrm{f}$. So at the next wave cycle the particle will then follow a different trajectory compared to the first cycle. Therefore each decorrelation can be regarded as a random jump, like in a Brownian motion. This is categorized as diffusion due to intrinsic stochasticity [Ukhorskiy and Sitnov, 2008].

Not only are there decorrelations between adjacent modes, but also between any modes in a spectrum. This time scale is:

$$
\tau_{\text {band }}=\frac{1}{f_{\max }-f_{\min }}
$$


During this time all modes in the spectrum initially shuffle the particles at a faster diffusion rate than the subsequent value derived from $\tau_{\Delta f}$ [Elskens, 2003].

Randomness can also be imposed onto the wave in the form of introducing a dynamic phase. This is also known as extrinsic stochasticity, which will have a diffusive time scale proportional to the randomization rate. It can be manifested in the physical environment in which the waves propagate, such as abrupt change in spatial dimensions along the propagation path, or random source pulses.

\subsubsection{The Diffusion Equation}

When there are random elements involved in wave-particle interactions, diffusion of the momenta and position is a possible outcome. The collective description of these quantities is via phase space density. Any of the three adiabatic invariants can be broken when changes in the magnetic field are sufficiently rapid. The implication is that the distribution of particle phase space densities, composed of the three main motion components: $f(\mu, J, \Phi)$ is affected. For each motion treated separately, the evolution is given by the Fokker-Planck equation:

$$
\frac{\partial f(S)}{\partial t}=-\frac{\partial}{\partial S}[\chi(S) f(S)]+\frac{\partial^{2}}{\partial S^{2}}[D(S) f(S)]+\text { source }- \text { loss }
$$

where $\mathrm{S}$ is the associated adiabatic invariant, and both sources and loss of $\mathrm{f}$ are included. The two coefficients in this equation are: the drag coefficient, $\chi$ and the diffusion coefficient, D. $\chi$ 
is the drag rate of the center of the distribution, while $\mathrm{D}$ is the expansion rate of the width of the distribution.

For long time scales when the first two adiabatic invariants are conserved Schulz and Lanzerotti [1974] established the diffusion equation for particles crossing Roederer L-shells:

$$
\frac{\partial f}{\partial t}=L^{* 2} \frac{\partial}{\partial L^{*}}\left[D_{L L} L^{*-2} \frac{\partial f}{\partial L^{*}}\right]-\text { loss }+ \text { source }
$$

The drag coefficient is assumed to be zero for all systems considered.

For an ensemble of particles that undergo radial scattering, the diffusion coefficient can be expressed simply as the rate of change of the variance:

$$
D_{L L}=\frac{\left\langle\left(\Delta L^{*}(\tau)\right)^{2}\right\rangle}{2 \tau}
$$

as $\mathrm{L}^{*}(\tau)$ for each particle deviates from either its initial or mean value, and $\tau$ is the characteristic diffusion time. In this dissertation $\tau$ will be defined as $\tau=1$ hour to give the unit $\left[D_{L L}\right]=h^{-1}$. The diffusion coefficient can also be converted to units of energy or drift frequency. Another diffusion also studied in radiation belt physics is in the pitch angle scattering, but this part is not central to this dissertation.

The diffusion coefficient can be estimated analytically, which for magnetospheric field fluctuations interacting with particles was established by Fälthammar (1965). Assuming a symmetric magnetic dipole, radial displacement is produced by time integration of the radial drift velocity for each particle. For an ensemble of particles, the expectation value of the displacement can then be calculated. The time derivative of the standard deviation is: 


$$
\frac{d}{d t}\left\langle\left(L-L_{0}\right)^{2}\right\rangle=2\left\langle\left(L-L_{0}\right) \frac{d L}{d t}\right\rangle=2\left\langle v(t) \int_{\xi=0}^{t} v(\xi) d \xi\right\rangle
$$

which is integrated:

$$
\left\langle\left(L-L_{0}\right)^{2}\right\rangle=\int_{\tau=0}^{t} \frac{d\left\langle\left(L-L_{0}\right)^{2}\right\rangle}{d t} d \tau=2 \int_{\tau=0}^{t} \int_{\xi=0}^{\tau}\langle v(\tau) v(\xi)\rangle d \xi d \tau
$$

The velocities would be the result from any radial drift motions and can be directly linked to the power spectral density of the wave fields. The variance of $L$ then gives $D_{L L}$ for an arbitrary time scale.

\subsubsection{Modeling the Diffusion Coefficient}

Numerous studies have been conducted in order to establish realistic diffusion rates, starting with Schulz and Lanzerotti [1974] who expanded their study for random fluctuations in either the electric or magnetic field, resulting in two different diffusion coefficients $\left(D_{L L}{ }^{E}\right.$ and $\left.D_{L L}{ }^{M}\right)$. Their method followed the same principle laid out by Fälthammar [1965] above, where ideal wave fields are integrated in a magnetic dipole, giving the results: $\mathrm{D}_{\mathrm{LL}}{ }^{\mathrm{E}, \mathrm{sym}} \alpha \mathrm{L}^{6}$ and $\mathrm{D}_{\mathrm{LL}}{ }^{\mathrm{M}, \mathrm{sym}} \alpha \mathrm{L}^{4}$. It is also found that the diffusion coefficient is directly related to the local power spectral density within the resonant band of the particle drift motion. Later on Fei et al. [2006] expanded the diffusion coefficient derivations to also encompass day-night

asymmetric dipole fields with sinusoidal compression term giving the relations: $\mathrm{D}_{\mathrm{LL}}^{\text {E,asym }} \alpha$ 
$\mathrm{L}^{12}$ and $\mathrm{D}_{\mathrm{LL}}^{\mathrm{M}, \text { asym }} \alpha \mathrm{L}^{10}$. Brautigam and Albert [2000] created an empirical model based on the Kp index which was verified for several studied events [Ozeke et al., 2012].

The diffusion coefficient has also been evaluated through observations and simulations. For example, Selesnick et al. [1997] found $D_{L L}$ directly via particle measurements from the Polar satellite over a 3 month period. Elkington et al. [2003] investigated the diffusion of test particles interacting with analytical fields - as described in chapter 3-2 - and found $\mathrm{D}_{\mathrm{LL}}$ to be measureable at time scales of geomagnetic storms. Huang et al. [2010] also employed test particles, but in an artificial MHD field with various solar wind speed inputs. The results in this paper backed up the earlier observed relation between particle flux enhancements and fast solar wind speed. Perry et al. [2006] is another example, where empirical fields based on ground magnetometer data were used in a model and confirming strong $\mathrm{D}_{\mathrm{LL}}$ dependence on the local power spectral density in L-shell. These various diffusion coefficients evaluated versus L-shells are shown in figure 2-4. 


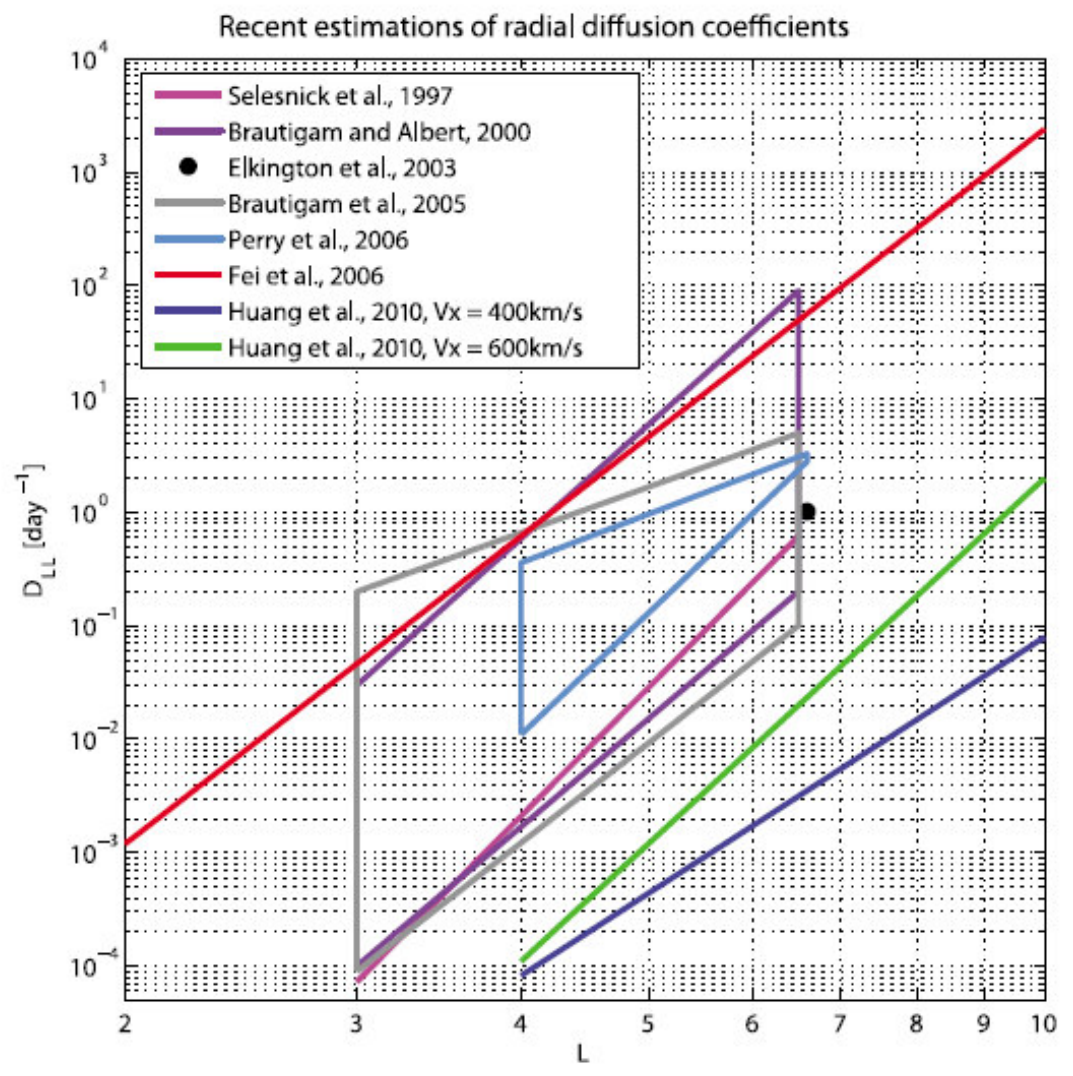

Figure 2-4: Estimations of the radial diffusion coefficient derived from models and observations [Huang et al., 2010]. 


\section{Introduction to ULF Waves}

\subsection{Magnetohydrodynamic Wave Theory}

Local or global perturbations can appear onto the magnetosphere, leading to electromagnetic ULF waves propagating along or across the magnetic field lines with velocities depending on a combination of the local Alfvén speed, $\mathrm{v}_{\mathrm{A}}$, and the sound speed, $\mathrm{v}_{\mathrm{S}}$, in the plasma medium [Gurnett and Bhattacharjee, 2005]. Since the phase velocity is dependent on plasma density the frequency can vary a great deal at different L-shells. The waves can also experience absorption and reflection as they reach sudden changes in local density. Apart from the ionosphere working as an absolute spatial boundary for wave propagation; the plasmasphere also plays a role in the wave evolution since it contains a significant plasma density within its boundaries.

The basic magnetohydrodynamic (MHD) equations that are used in this wave theory are:

$$
\begin{gathered}
\bar{\nabla} \times \mathbf{B}=\mu_{0} \mathbf{J} \\
\bar{\nabla} \cdot \mathbf{B}=0 \\
\nabla \times \mathbf{E}=-\partial \mathbf{B} / \partial t \\
\bar{\nabla} \cdot \mathbf{E}=0 \\
\frac{\partial \rho_{m}}{\partial t}+\bar{\nabla} \cdot\left(\rho_{m} \mathbf{U}\right)=0
\end{gathered}
$$




$$
\begin{gathered}
\rho_{m} \frac{\partial \mathbf{U}}{\partial t}=\mathbf{J} \times \mathbf{B}-\bar{\nabla} P \\
\mathbf{J}=\sigma(\mathbf{E}+\mathbf{U} \times \mathbf{B}) \\
\frac{d}{d t}\left(P \rho_{m}^{-\gamma_{a}}\right)=0
\end{gathered}
$$

where $\rho_{\mathrm{m}}$ is the mass density, $\mathbf{U}$ is the fluid velocity and $\mathrm{P}$ is the isotropic pressure. The power index $\gamma_{\mathrm{a}}$ is an adiabatic measure of rapid changes in the local frame. To evaluate these equations for waves, i.e. perturbations in the system, one needs to linearize them to a first order. Assuming a zeroth-order magnetic field being $\mathrm{B}=\mathrm{B}_{\mathrm{z} 0}$, then if one rewrites these first order equations in terms of the fluid velocity and the wave propagation speed, $\mathrm{v}_{\mathrm{p}}$, a resulting set of equations is written in a matrix form:

$$
\left[\begin{array}{ccc}
v_{p}^{2}-v_{S}^{2} \sin ^{2} \theta-v_{A}^{2} & 0 & -v_{S}^{2} \sin \theta \cos \theta \\
0 & v_{p}^{2}-v_{A}^{2} \cos ^{2} \theta & 0 \\
-v_{S}^{2} \sin \theta \cos \theta & 0 & v_{p}^{2}-v_{S}^{2} \cos ^{2} \theta
\end{array}\right]\left[\begin{array}{l}
U_{x, 1} \\
U_{y, 1} \\
U_{z, 1}
\end{array}\right]=0
$$

which roots are:

$$
\begin{gathered}
v_{p}^{2}=\frac{1}{2}\left(v_{A}^{2}+v_{S}^{2}\right)+\frac{1}{2}\left[\left(v_{A}^{2}-v_{S}^{2}\right)^{2}+4 v_{A}^{2} v_{S}^{2} \sin ^{2} \theta\right]^{1 / 2} \\
v_{p}^{2}=\frac{1}{2}\left(v_{A}^{2}+v_{S}^{2}\right)-\frac{1}{2}\left[\left(v_{A}^{2}-v_{S}^{2}\right)^{2}+4 v_{A}^{2} v_{S}^{2} \sin ^{2} \theta\right]^{1 / 2} \\
v_{p}^{2}=v_{A}^{2} \cos ^{2} \theta
\end{gathered}
$$


The angle $\theta$ is the direction of the wave vector on a perpendicular plane with respect $B_{0 z}$. Depending on the selection of root the resulting vector components of $U_{1}, E_{1}$ and $B_{1}$ will differ. For the root (3.1.12) for any $\theta \neq \pi / 2+n \pi$, the resulting eigenvectors become $\mathbf{U}_{1}=\left(0, U_{y 1}, 0\right), \quad \mathbf{B}_{1}=\left(0, B_{y 1}, 0\right), \quad \mathbf{E}_{1}=\left(E_{x 1}, 0,0\right)$ and $\rho_{m, 1}=0$, thus the wave is purely electromagnetic with a Poynting vector and group velocity parallel to $\mathrm{B}_{0 \mathrm{z}}$. This is better known as an Alfvén wave.

The other roots of (3.1.10) and (3.1.11) consist of both $\mathrm{v}_{\mathrm{A}}$ and $\mathrm{v}_{\mathrm{S}}$. The sound wave component for $\mathrm{v}_{\mathrm{S}}$ is known as a compressional mode. The $1^{\text {st }}$ order oscillations are known as magnetosonic waves for any combination of $v_{\mathrm{A}}$ and $\mathrm{v}_{\mathrm{S}}$. These waves group into a "fast mode" when $\mathrm{v}_{\mathrm{A}}>\mathrm{v}_{\mathrm{S}}$ and "slow mode" when $\mathrm{v}_{\mathrm{S}}<\mathrm{v}_{\mathrm{A}}$. The eigenvectors are dependent on $\theta$, but when $\mathrm{v}_{\mathrm{S}} \gg \mathrm{v}_{\mathrm{A}}$ the electromagnetic pointing flux along the field line approaches zero and the wave propagation becomes directed perpendicularly to the field line.

Compressional and shear Alfvén waves can be generated by several sources, inside or outside the magnetosphere. The most well-known drivers will be described in the following paragraphs.

\subsection{Magnetosonic Waves}

One type of perturbation imposed on the magnetosphere is due to Kelvin-Helmholtz (KH) instability. This is a fluid mechanics phenomenon that takes place between two layers of fluid with different parallel velocities and densities when the instability criterion is met. A restoring 
force, for example tension force in fluids, holds back the instability as long as the perturbation is sufficiently small. In magnetohydrodynamics the magnetic field is the restoring tension force. The two layers in question here are the magnetosphere outer boundary and the magnetosheath with a strongest effect at the magnetic equator. The instability condition is:

$$
\left(v_{\text {sphere }}-v_{\text {sheath }}\right)^{2}>\frac{\left(\mathbf{B}_{\text {sheath }} \cdot \hat{k}\right)^{2}}{\mu_{0} \rho_{\text {sphere }}}
$$

For the onset of instability, surface ripples appear along the magnetopause and propagate tailward and radially inward. The most suitable solar wind conditions for wave generations of this kind are when IMF > 0 , so that the magnetosphere does not undergo any significant topology changes, and a high speed stream.

Interplanetary space can also contain ULF waves carried by the solar wind, originating from the Sun. These waves buffet the dayside magnetopause via pressure pulses, either originating directly from the solar wind [Barnes, 1983] or from the bow shock defining the outer boundary of the plasma sheath [Greenstadt, 1980]. Identical frequencies have been observed in compressional waves within the magnetosphere to demonstrate a direct transfer of modes [Stephenson and Walker, 2002; Kepko and Spence 2003]. There is evidence that solar wind pulses drive about half of all ULF activity within the magnetosphere [Viall et al., 2009].

Another source of ULF waves is suggested to be in the tail due to perturbations from convectively flowing bulks of plasma being injected toward Earth from substorms [Kivelson, 2006 review]. These waves are classified in the Pi-2 category. It is thought that the waves can be generated either locally in the frozen-in flux tubes flowing along the bulk plasma as the group passes through different surrounding plasma in the inner magnetosphere, or that the 
source is farther up in the tail and create waves that propagate earthward. The waves are compressional and can propagate both across the field lines, as well as along them. Since substorms cause auroral brightening at Earth's upper atmosphere there is ongoing research in the role of the waves in accelerating particles precipitating into the auroral zones [Rae et al. 2012].

\subsection{Alfvén Waves \& Field Line Resonances}

The transverse Alfvén modes [Alfvén 1942] have a distinct phase velocity that depends on both the magnetic intensity and plasma density, similar to a musical string that depends on the tension force and the mass density. A more common name for these waves is field line resonances, which is derived from a resonant coupling with a magnetosonic mode wave vector. The end points of the oscillating field lines are tied to the ionosphere since it is a conducting surface. While Alfvén established the wave theory, Dungey [1963] provided various solutions to these hydromagnetic waves in a dipolar setting with an ionosphere. Assuming a 2D box model of the magnetic equator, it becomes straightforward to find an MHD solution [Degeling, 2006], [Southwood 1974], [Chen and Hasegawa, 1974a], [Radoski, 1976].

In order to derive the condition for coupling the first-order linearized cold plasma MHD equations are used:

$$
\rho \frac{\partial \mathbf{v}_{1}}{\partial t}=\mathbf{J}_{1} \times \mathbf{B}_{0}
$$




$$
\begin{gathered}
\mu_{0} \mathbf{J}_{1}=\nabla \times \mathbf{B}_{1} \\
\frac{\partial B_{1}}{\partial t}=-\nabla \times E_{1} \\
E_{1}=-v_{1} \times B_{0}
\end{gathered}
$$

Combining these equations gives:

$$
\frac{\partial^{2} b_{z}}{\partial L^{2}}-\left(\frac{\partial K^{2} / \partial L}{K^{2}-k_{z}^{2}}-\frac{1}{L}\right) \frac{\partial b_{z}}{\partial L}+\left(K^{2}-k_{z}^{2}\right) b_{z}+\frac{1}{L^{2}} \frac{\partial^{2} b_{z}}{\partial \phi^{2}}=0
$$

where $\mathrm{k}_{\mathrm{z}}$ is the wave vector for the shear wave that spans along the field lines with endpoints at the ionosphere, and

$$
K(L, \phi)=\frac{\omega}{v_{A}(L, \phi)}
$$

belongs to the compressional mode. The frequencies of these two modes match at a particular radius where the solution becomes singular. This PDE can be decoupled into $r$ and $\varphi$ by omitting the $\varphi$-dependence for $\mathrm{v}_{\mathrm{A}}$. A solution is numerically found in this simple box model for the two decoupled variables where the particular parameters $r_{\mathrm{FLR}}=5$ and $\mathrm{m}_{0}=2$ are used (fig 4-14). 

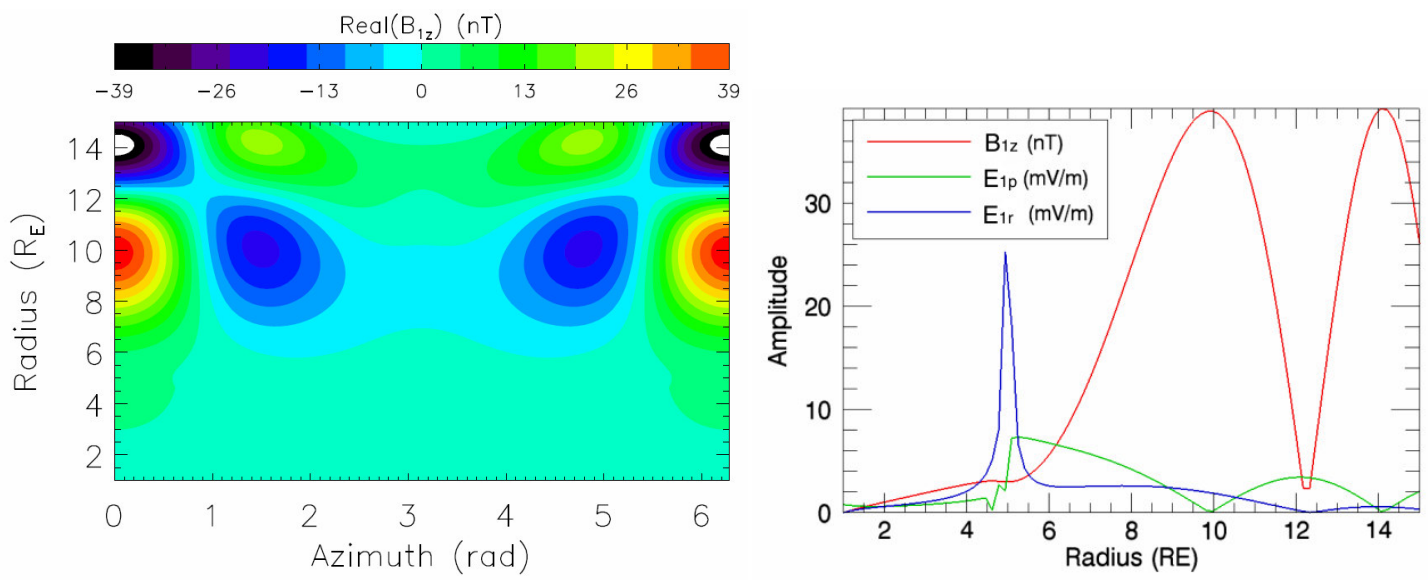

Figure 3-1: Spatial 2D box model solution to the MHD equations with coupled wave vectors leading to a field line resonance.

For the two lowest harmonics oscillating at the local eigenfrequency there is either an odd or even mode in the magnetic wave component, as depicted in figure 3-1. Since these waves tend to be steady for long periods of time they are classified as Pc modes. Oscillations including a shift in field lines along the azimuthal direction are called toroidal modes, and the shifts in radial direction are poloidal modes. From Faraday's law, electric field components accompany these waves as well, where the toroidal mode yields a vector of $\mathbf{E}_{\mathbf{0}}=E_{\phi 0} \hat{\boldsymbol{\phi}}$, and the poloidal mode $\mathbf{E}_{\mathbf{0}}=E_{L 0} \hat{L}$. 


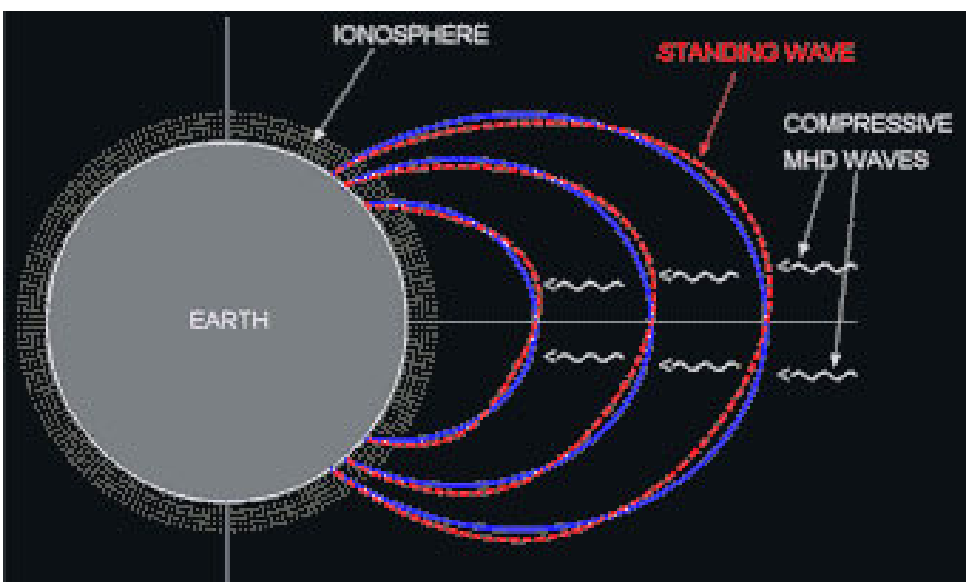

\section{Toroidal $\rightarrow \mathrm{E}_{\phi}$-component Poloidal $\rightarrow \mathrm{E}_{\mathrm{L}}$-component}

North
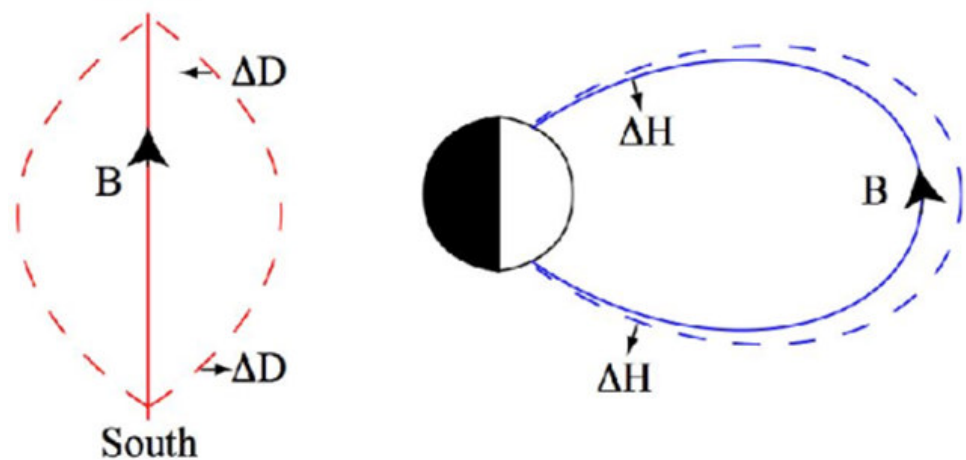

South

Figure 3.2: Top: General depiction of field-line resonance excitation by compressional waves. Bottom: Fundamental field-line resonance modes [Hughes, 1994].

Returning the discussion to the standing field line resonances, a trigger is needed to onset such activity. Compressional waves fulfill this role [Takahashi, 1988 (Sw pressure); Chen and Hasegawa, 1974; Southwood, 1974; Kivelson and Southwood, 1985]. The amplitude from the driver waves transfers into the standing mode. Field line resonances can be steady for time scales over an hour when the plasma density along the magnetic flux tube remains constant. The wave power dissipates slowly into the ionosphere. Compressional waves however cross various plasma elements, and also are very dependent on the magnetopause location, hence dissipation and phase mixing occur faster than for FLRs. This suggests that external driving is essential to maintain such ULF activity within the magnetosphere [Kivelson, 2006 review]. 
Research has also shown that it is possible to for standing waves to appear from compressional waves reflecting between the plasmapause and the magnetopause [Mann and Wright, 1995]. This is also a feature seen in the MHD case study in chapter 6 of this dissertation.

\subsection{Statistical Maps of ULF Wave Occurrence and Amplitude}

Observational statistical studies have indicated the existence of ULF waves due to all mechanisms discussed above. With the satellite AMPTE/CCE magnetometer data over a total sampling time of 7231 hours, Anderson et al. [1990] mapped the ULF activity occurrence rate for different amplitude levels and reached the conclusion that the wave activity was most prominent at the dusk and dawn sectors of the magnetosphere, and the latitudinal distribution has over $80 \%$ of wave occurrence at latitudes less than $13^{\circ}$ away from the magnetic equator (see fig. 3-3). Their argument is made for Kelvin-Helmholtz instability as the main driver for ULF waves in the magnetosphere from this due to the spatial distribution concentrated at the flanks. A later study by Liu et al. (2009) composed statistical maps of wave electric fields with THEMIS satellite data collected over a 13-month period. Their result is consistent with a focus of wave activity at the equatorial flanks, but other details reveal wave electric fields existing at higher L-shells in the dayside sector hinting on solar wind impulses as the main driver (see fig. 2.2) in more agreement with Viall et al. (2009). 


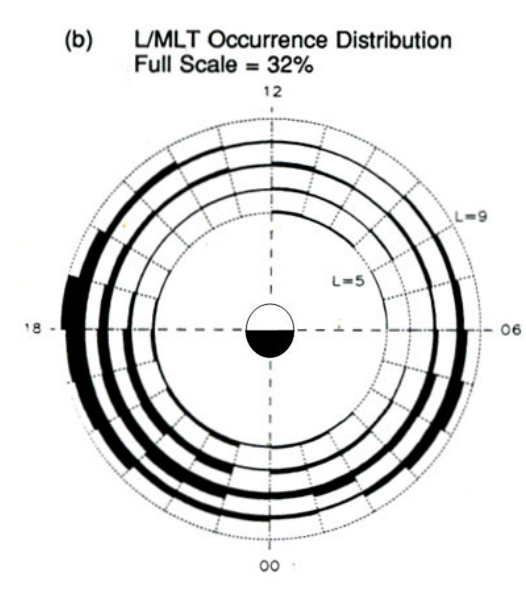

(c) MLAT Distribution
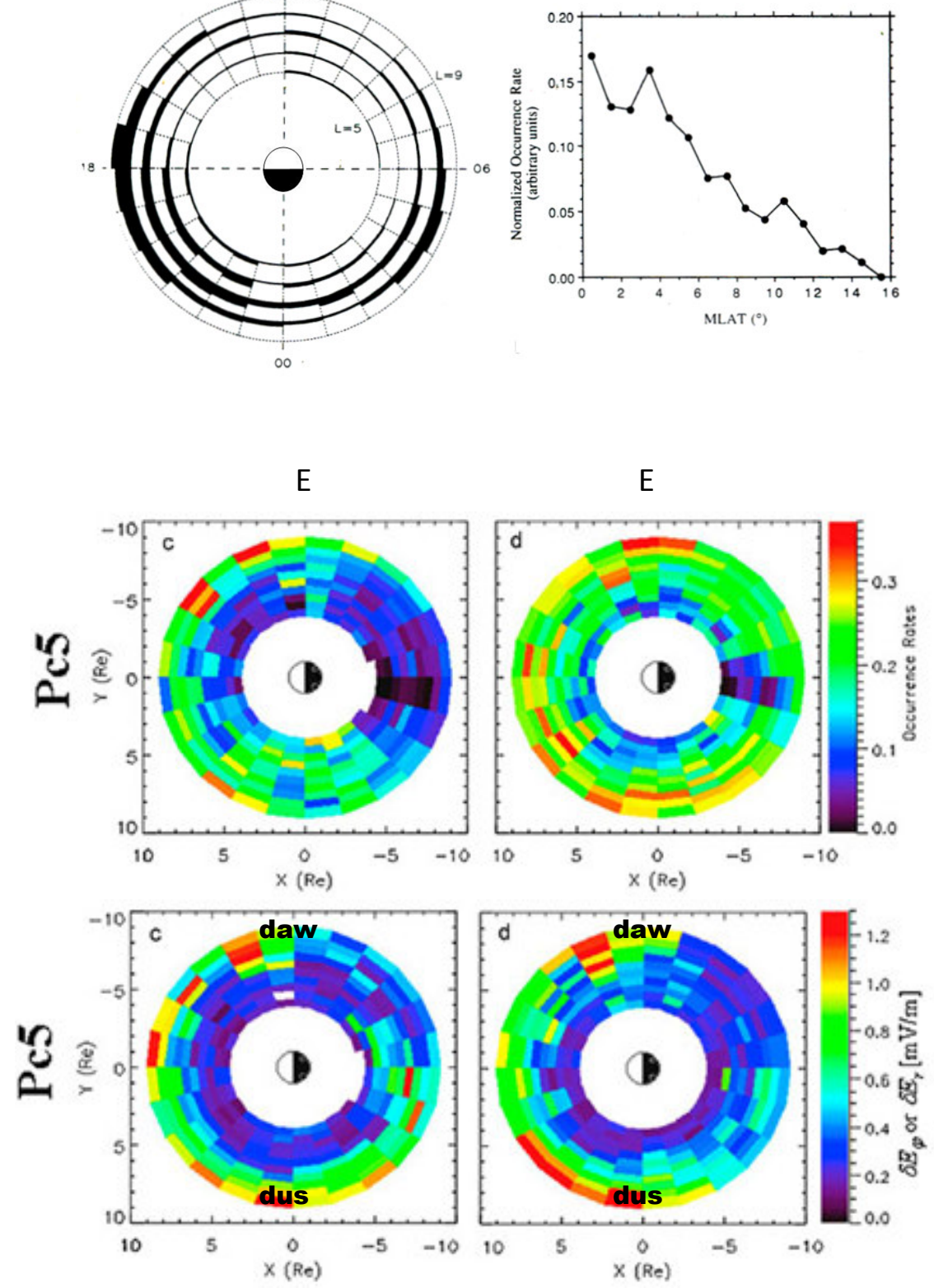

Figure 3-3: Upper left: Global occurrence rate map for Pc5 wave magnetic fluctuations [Anderson, 1990].

Upper right: Occurrence rate distribution in magnetic latitude [Anderson, 1990].

Middle \& Lower: Global occurrence rate and average amplitude of wave electric fields [Liu, 2009]. 
In summary, field line resonances tend to be relatively strong and localized in magnetic local time due to precise eigenfrequencies between the endpoints in the magnetic dipole.

Compressional modes have been observed and modeled at all magnetic local times depending on the generator mechanism. Irregular Pi waves tend to appear in the midnight sector, $\mathrm{KH}$ waves at the flanks, and pulses originating from outside the magnetopause propagating into the dayside. 


\section{Wave-Particle Interactions}

In this chapter we present detailed results in adiabatic electron scattering due to interactions with monochromatic ULF waves, building on the basic discussion of chapter 2-3. We separate the scattering process into a local and global phenomenon, determined by the initial conditions for the electrons. We investigate the wave parameters: phase, propagation coordinates, and polarization, in terms of the effect on particle scattering. We show that 2wave systems with overlapping resonances are capable of producing global acceleration, while single waves are not.

\subsection{Introduction}

The discussion we gave in chapter 2.2 contained one example of a monochromatic wave interacting with electrons set to a uniform first adiabatic invariant $\mu$. In reality, outer belt electrons have a range of kinetic energies and are spread uniformly along all MLTs. We will consider this expansion of initial conditions to evaluate what the local and global effects are in short-term ( $\tau \sim$ hours $)$ wave-particle interactions. Our discussion here will at first repeat a similar case as in 2.2 , but for a wave $\mathrm{E}_{\phi}$ field in an azimuthally symmetric magnetic dipole which is in fact a more straightforward case. Once we have established results that agree with the discussions in 2.2 , we will methodically evaluate wave parameters such as phase and polarization and their effects on scattering. Knowing which wave parameters have largest effect can be useful in future work involving observations or modeling of ULF waves in the 
magnetosphere. Systems containing more than one simultaneous, monochromatic wave with overlapping resonance widths are not well understood, mainly because particle trajectories become chaotic. As part of this chapter we will demonstrate the difference between 1-wave and 2-wave systems in terms of both local and global electron scattering.

\subsection{Electron Scattering by a Monochromatic Wave $\mathbf{E}_{\phi}$ Field}

The general form of an electric wave field that we use in this chapter is a standing wave:

$$
\mathbf{E}(\phi, t)=\mathbf{E}_{\mathbf{0}} \cos \left(\omega t+\varphi_{1}\right) \cos \left(m \phi+\phi_{0}\right)
$$

where there are two phases: $\varphi_{1}$ and $\phi_{0}$, that represent the temporal and spatial component respectively. We let these waves span the entire local time, 00:00 $<$ MLT $<24: 00$, in all sections of this chapter except the last one in 4.5 where they are confined in MLT sectors.

Throughout this chapter we use a compressed dipolar magnetosphere model similar to equation (2.1.21):

$$
B(L)=\frac{B_{0}}{L^{3}}+B_{c} \cos \phi
$$

where $\mathrm{B}_{0}=30,500 \mathrm{nT}-$ the field amplitude at Earth's surface at the magnetic equator. The compression term $\mathrm{B}_{\mathrm{c}}$ is a constant value that in this study we use either $\mathrm{B}_{\mathrm{c}}=0 \mathrm{nT}$ or $\mathrm{B}_{\mathrm{c}}=30$ nT. 
We will also use radians as the unit for phases between waves and particles for all cases where there is no geomagnetic spatial reference, e.g. a symmetric magnetic dipole field.

In this section the dipole field is symmetric, i.e. $\mathrm{B}_{\mathrm{c}}=0 \mathrm{nT}$; only the azimuthal component, $\mathrm{E}_{\phi}$, of the wave electric field is then capable of scattering electrons. In this case the corresponding change in energy for a particle interacting with this standing wave is similar to that of the propagating wave interaction as described in chapter 3-2:

$$
\begin{aligned}
& W-W_{0}=\frac{3 \gamma_{m} \mu}{L_{0}} \int E_{\phi}(\phi, t) d t= \\
& =\frac{3 \gamma_{m} \mu}{L_{0}} \frac{E_{\phi 0}}{2} \frac{\left[\left(\omega-m \omega_{d}\right) \cos \left(\left(\omega+m \omega_{d}\right) t^{\prime}+\varphi_{1}+\phi_{0}\right)-\left(\omega+m \omega_{d}\right) \cos \left(\left(\omega-m \omega_{d}\right) t^{\prime}+\varphi_{1}-\phi_{0}\right)\right]}{\left(\omega^{2}-m^{2} \omega_{d}^{2}\right)}
\end{aligned}
$$

for which the resonant condition is

$$
\pm m \omega_{d}=\omega
$$

For $m \omega_{d} \rightarrow \omega$ the energy scattering in equation (4.2.3) approaches:

$$
W-W_{0} \rightarrow-\frac{3 \gamma_{m} \mu}{L_{0}} \frac{E_{\phi 0}}{2} \frac{\left[\cos \left(\left(\omega-m \omega_{d}\right) t^{\prime}+\varphi_{1}-\phi_{0}\right)\right]}{\left(\omega-m \omega_{d}\right)}
$$

which is the same form as the propagating wave.

Since the background field is azimuthally symmetric and the wave field is distributed globally (i.e. over all local times), it is redundant to add another azimuthal phase due to particle initial position. The relative phase between the wave and the particles can be summarized into a single parameter: 


$$
\varphi(t)=\left(m \omega_{d}-\omega\right) t+\varphi_{1}-\phi_{0}=\left(m \omega_{d}-\omega\right) t+\varphi_{0}
$$

Rewriting equations $(2.1 .14)$ and $(2.1 .15)$ by considering the relative phase, the motion description becomes:

$$
\begin{gathered}
\dot{L}=\frac{E_{0}}{B_{0}} L(t)^{3} \cos \left(\phi(t)+\varphi_{1}\right) \\
\dot{\phi}=\frac{3 \gamma_{m} \mu}{L^{2}(t)}-\omega
\end{gathered}
$$

These coupled differential equations are numerically solvable for any initial condition provided that the first adiabatic invariant $\mu$ is fixed for any initial condition. We then initiate this discussion by setting the initial conditions for the electrons over distribution in L-shell $\left(\mathrm{L}_{0}=4.0-6.0\right)$ which leads to a set of energies $\left(\mathrm{W}_{0} \approx 1.0-2.4 \mathrm{MeV}\right)$ via Eq. (2.1.3). The distribution in azimuth $\left(\phi_{1}=0-2 \pi\right)$ has no effect on the adiabatic invariant here since the magnetic dipole is assumed symmetric $\left(B_{c}=0 \mathrm{nT}\right)$. The top figure $4-1$ shows the solution for a total time interval of $\tau=6.7$ hours. This time span is equivalent to a recurrence period $\tau_{\mathrm{w}-\mathrm{d}}$ (Eq. (2.2.9)) for $\mathrm{f}_{\mathrm{d}}-\mathrm{f}_{1}=0.04 \mathrm{mHz}$, which is sufficient to cover a whole wave-particle interaction cycle for most electrons. In the figure we converted the L-shells to perpendicular energies due to $\mu$. We set the monochromatic wave at a frequency $\omega_{1} / 2 \pi=1.87 \mathrm{mHz}$ and an amplitude $\mathrm{E}_{\phi 0}=0.3 \mathrm{mV} / \mathrm{m}$, so that the main resonance band among the drift frequencies is covered by a reasonable margin. We assume an azimuthal wavenumber of $\mathrm{m}=1$ and a wave phase of $\phi_{1}=\pi / 2$ in order to set the separatrix at $\phi=0$ for the resonant frequency.

As the system becomes more complex with asymmetric dipole or multiple waves, a test particle simulation provides a good approximation of responses in wave-particle interactions. 
For the remainder of this chapter, as well as large portions of chapters 5 and 6 , we use a $4^{\text {th }}$ order Runge-Kutta procedure. Appendix A discusses the details of this code. For the case of a fixed $\mu$ we used the guiding-center simulation and compared to the solution of (4.2.7) and (4.2.8). The initial parameter ranges are the same as for the system of ODEs above, but with a higher resolution: $\Delta \phi=2 \pi / 60$ and $\Delta \mathrm{L}=0.05$.

Visible in figures 4-1a and c, a clear distinction appears in phase space trajectories depending on the initial condition, where the electrons end up in either open curves propagating with an either increasing or decreasing phase with respect to the wave, or closed curves depicting a trapping in phase. The separatrix defines what initial conditions a particle must have in order to either overcome the wave field potential, or become trapped. As equation (4.2.5) states, the wave can be approximated as propagating with electrons drifting azimuthally along the wave front. The relative phases of two selected electrons with different initial conditions are shown in figure 4-1b. Both start in phase with positive wave amplitude and with higher azimuthal drift frequencies than the wave frequency. For $\mathrm{E}>0 \mathrm{mV} / \mathrm{m}$ the radial drift velocity $\dot{L}>0 s^{-1}$ leading to $\dot{\varphi}<0 \mathrm{rad} / \mathrm{s}$, thus both electrons slow down initially. The electron with $\mathrm{W}_{0}=1.79$ $\mathrm{MeV}$ eventually slows down to reversed direction in relative phase velocity and then heads to the opposite turning point where the amplitude is negative, effectively bouncing back and forth across the single node at $\phi=\pi$. The other electron at $\mathrm{W}_{0}=1.90 \mathrm{MeV}$ does not reach the point of reversal and instead crosses the node at $\phi=2 \pi$ and continues to drift faster than the wave propagation speed. Electrons drifting above the wave frequency propagate to the right in the phase space diagram, and vice versa to those below the resonance. An electron with an initial drift frequency exactly at $f_{d}=f_{1}$, launched at either fixed point $\varphi_{i}=0$ or $\pi$, will neither 
gain nor lose any energy. The maximum extent of scattering is the resonance island width found by the equations (2.2.10), (2.2.11) and (2.2.12).
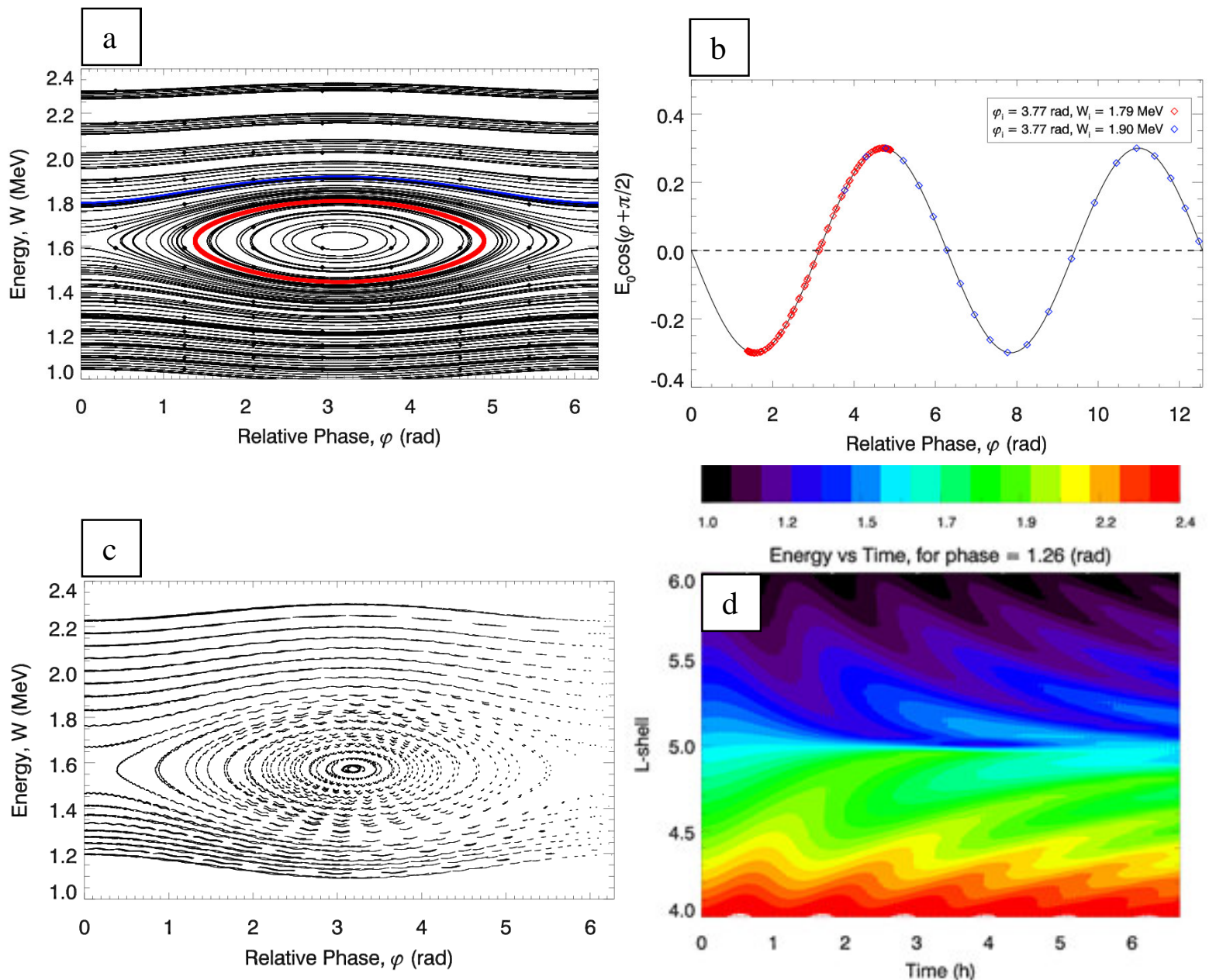

Figure 4-1: Electrons interacting with a monochromatic, $E_{\phi}$ wave field in a symmetric dipole field. The $1^{\text {st }}$ adiabatic invariant $\mu$ is uniform and conserved for all initial conditions. Top row: Solution to equations (4.2.7) and (4.2.8). Bottom row: Result from a test particle simulation. a) and c) are the phase space trajectories for all particles. $b$ ) is the phase space coordinates of two electrons from the solution with their relative phase to the wave sampled at $\left.T_{s}=250 \mathrm{~s} . \mathrm{d}\right)$ shows the energy evolution for the test particles in the simulation. 
In reality, outer belt electrons can have a large range of adiabatic invariant values, which makes the solution of the equations (4.2.7) and (4.2.8) unique for each $\mu$. To understand the implications in local acceleration, we simulate additional sets of electrons with initial energies $\mathrm{W}_{0}=1-2 \mathrm{MeV}(\Delta \mathrm{W}=0.01 \mathrm{MeV})$, but at an initial L-shell at $\mathrm{L}_{0}=5$ giving different $\mu$ values. Through equation (2.1.16) these initial conditions correspond to a range of drift frequencies: $\omega_{\mathrm{d}} / 2 \pi=1.3-2.3 \mathrm{mHz}$. Three such simulations (case \#1, \#2 and \#3) with initial azimuthal positions $\varphi_{1}=\pi / 2,3 \pi / 2$ and $\pi$ in respective order, are conducted and shown in figure 4-2. The graphs on the left display the time-dependent evolution of the particle energies, and the graphs on the right show phase space diagrams for Poincare surface of section with a sample rate of $f_{\text {sampling }}=f_{1}=1.87 \mathrm{mHz}$ for 4 selected, resonant electrons. A group of 7 electrons just above the resonant frequency are marked in colors in each phase space graph. Once again open and closed phase space trajectories appear depending on the choice of initial wave phase. 

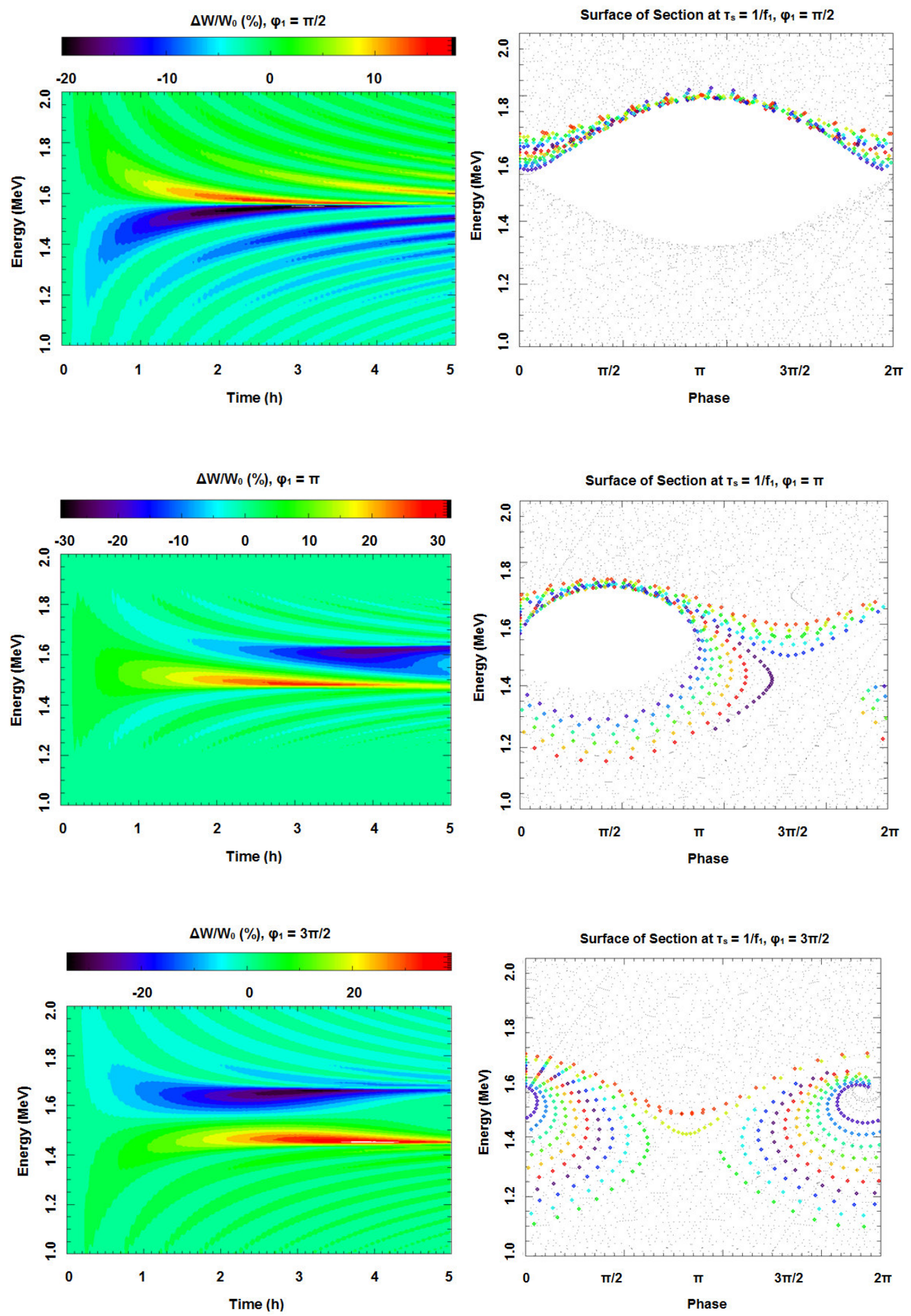

Figure 4-2: Electron energy change due to interaction with an $E_{\phi}$ wave. Left: Energy as a function of time. Right: Poincare surface of section of electron phase space sampled at $T_{s}=T_{w}$. The phases are at top: $\phi_{0}=\pi / 2$, middle: $\phi_{0}=\pi$, and bottom: $\phi_{0}=3 \pi / 2$. 
Instead of assigning wave phases, one can also initially distribute the electrons across all azimuthal coordinates. It is in fact more realistic since particles in each energy level are always distributed across all MLTs in the radiation belt. We apply a phase $\phi_{1}=\pi / 2$ to a monochromatic wave of the same type as previous examples, and distribute electrons with 1.0 $<\mathrm{W}_{0}<2.2 \mathrm{MeV}\left(\Delta \mathrm{W}_{0}=0.05 \mathrm{MeV}\right)$ and $0<\varphi_{0}<2 \pi\left(\Delta \varphi_{0}=0.1 \mathrm{rad}\right)$. This global acceleration process is shown in a Poincare surface of section map in figure 4-3.

Particles launched near the fixed, unstable point at $\phi_{0}=\pi / 2$ end up following open ended trajectories. If on the other hand the initial condition is $\phi_{1} \neq 0+n \cdot 2 \pi$, for any $n$, there are both open and closed phase space trajectories depending on the initial energy. Each $\mathrm{W}_{0}$ now leads to unique trajectories that can overlap in phase space. These overlaps occur between electrons launched on opposite sides of the resonant energy. The closed loops are also asymmetric on opposite side of the resonance due to the different $\mu$ associated with each initial electron energy value.

We emphasize the difference between local and global acceleration of particles. Global acceleration takes all initial conditions into account, while a local acceleration only considers one initial MLT. In figure 4-2, left column, we see examples of local acceleration. There is a clear distinction between electrons launched above and below the resonance frequency. However, an averaged energy shift for all the initial MLTs (see figure 4-3, bottom) is not as asymmetric around the resonance. Electron energies undergo largest changes over a period of $\sim 2$ hours within each recurrence period, which can have implications on the final energy distribution when the wave dissipates at any time during the scattering process. The global population experience negligible change in energy. 

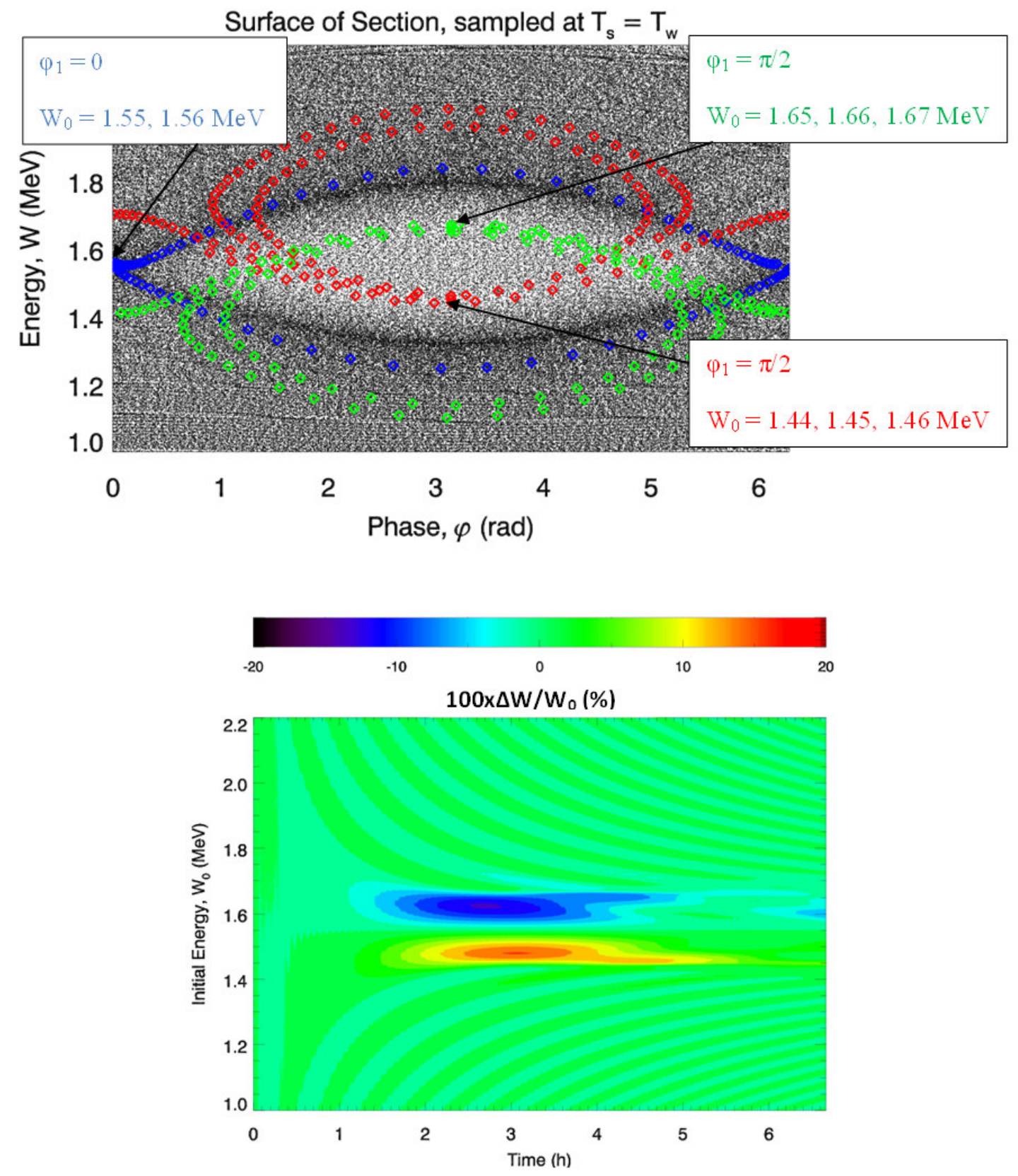

Figure 4-3: Electron energy scattering due to interaction with monochromatic wave electric field with $E_{\phi}$ component. The electrons are initially distributed uniformly in relative phase to the wave. Top: A Poincare surface of section of electron phase space at $T_{s}=T_{w}$ is plotted for three different adiabatic invariants and initial azimuthal positions. Bottom: Averaged energies for all initial MLTs. 
The maximum $\Delta \mathrm{L}$ defining the resonant width in phase space can only be achieved for a particle that is set to an initial drift frequency just above or below the resonance for $\phi=\pi / 2$ and at the outer boundary of the resonant band for $\phi=3 \pi / 2$. We find from the above simulation, by using equation (2.2.10) and an initial, resonant energy of $\mathrm{W}_{0}=1.55 \mathrm{MeV}$ (i.e. resonant drift frequency), that the maximal radial shift is $\Delta \mathrm{L}=0.53$. The extreme values found from the simulation data show: $\Delta \mathrm{L}_{+}=0.62$ and $\Delta \mathrm{L}_{-}=-0.42$, giving an average of $\Delta \mathrm{L}=$ 0.52. The corresponding values for particle energy and drift frequency changes are calculated to: $\Delta \mathrm{W}=0.31 \mathrm{MeV}$ and $\Delta \mathrm{f}=0.11 \mathrm{mHz}$. From the simulation these values are found: $\Delta \mathrm{f}_{+}=$ $0.12 \mathrm{mHz}, \Delta \mathrm{f}_{-}=-0.11 \mathrm{mHz}, \Delta \mathrm{W}_{+}=0.33 \mathrm{MeV}$, and $\Delta \mathrm{W}_{-}=-0.26 \mathrm{MeV}$. The numbers are most accurate for the open phase space trajectories, whereas for the closed trajectories there is a strong asymmetry in the positive and negative maximal scattering. Thus for any phase the maximal scattering, i.e. resonant width, is approximately $\left|\Delta_{+}\right|+\left|\Delta_{-}\right| \approx|2 \Delta|$.

For the same wave amplitude we calculate the upper half of the resonant band in energy and frequency for a set of different initial L-shells and wave frequencies using equations (2.2.10),

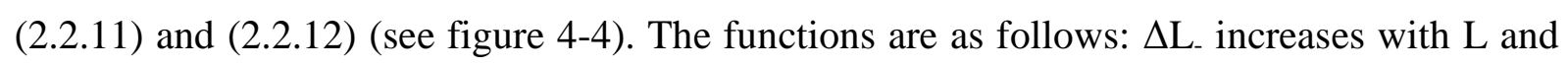
decreases with $f_{d}, \Delta f_{+}$increases with both $L$ and $f_{d}$, and $\Delta W_{+}$increases with $f_{d}$. Thus effective energy scattering can be expected at high L-shells and high initial energies - especially $\Delta \mathrm{f}_{+}$is an important parameter when multiple waves are present, which will be discussed in detail in section 4-3, as well as chapters 5 and 6 . In contrast, radial transport is more efficient when particles interact with waves at relatively low frequencies within the Pc-5 range. 

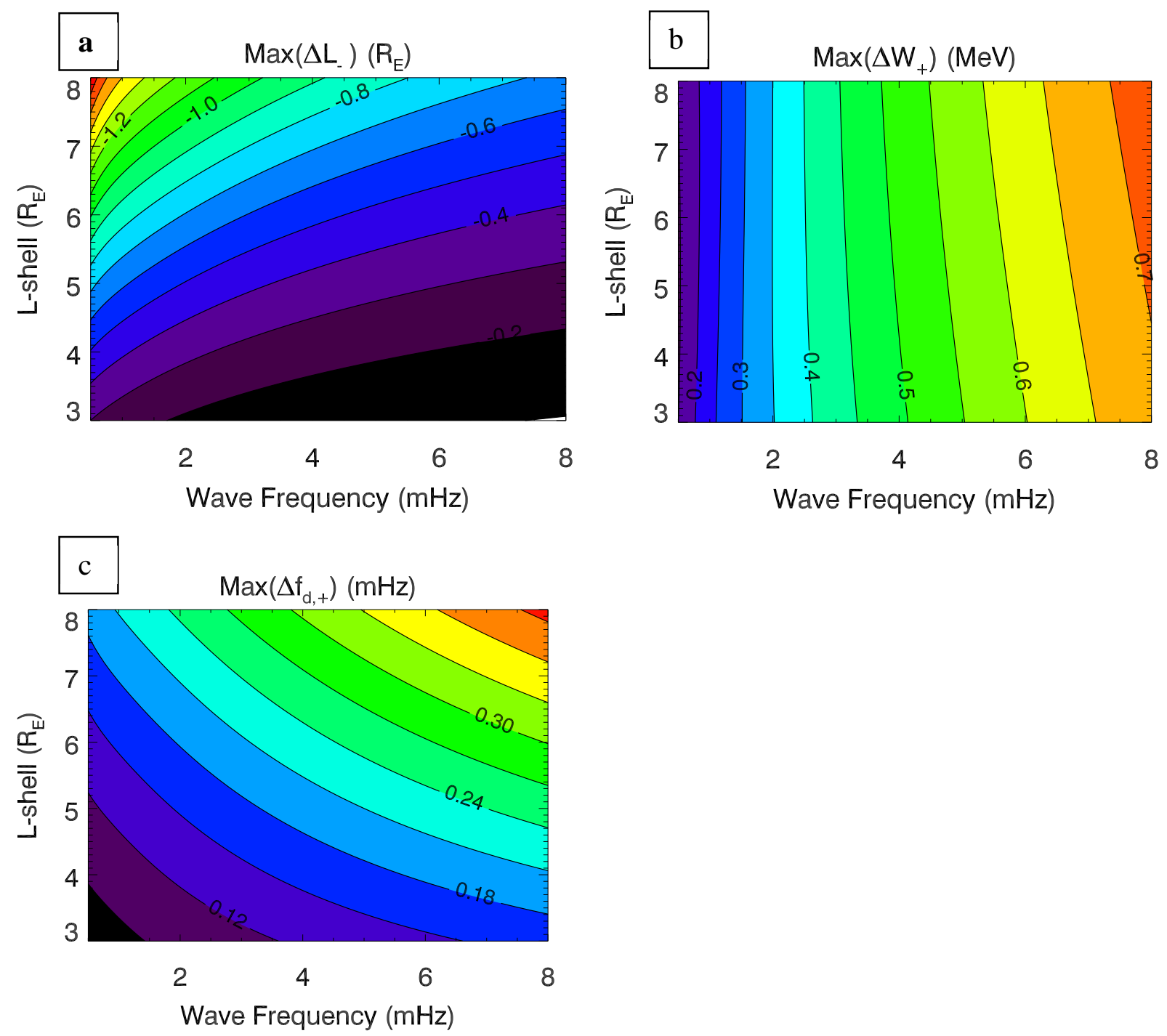

Figure 4-4: Calculations of maximum scattering in a) L-shell, b) drift frequency, and c) energy, from equations (2.2.10), (2.2.11) and (2.2.12) for a monochromatic wave $E_{\phi}$ field. 


\subsection{Electron Interactions with a Monochromatic Wave $\mathbf{E}_{\mathrm{L}}$ Field}

The radial component $\mathrm{E}_{\mathrm{L}}$ in the wave electric field can also cause electron scattering in the radiation belt via equation (2.1.15). We will continue investigating the case for the compressed dipole field, as first discussed in the introduction in chapter 2-2 [Elkington et al., 1999], with more details. Here we assume an eastward propagating wave of the form (4.2.1):

$$
E_{L}(\phi, t)=E_{0} \cos \left(\omega t+\varphi_{1}\right) \cos \left(m \phi+\phi_{0}\right)
$$

with radial polarization and an azimuthal wave vector in a magnetospheric field modeled as a solar wind-compressed magnetic dipole field of Eq. (4.2.2) with $\mathrm{B}_{\mathrm{c}}=30 \mathrm{nT}$. By including a compression term, along with a wave electric field, the particle trajectories become more complex. The cases with the single wave immersed in a symmetric dipole only had one free parameter - that of the relative phase between the wave and the particle. For the asymmetric dipole there are 3 free parameters: the position in MLT for the particle $\left(\varphi_{0}\right)$, the relative phase between the particle and the wave $\left(\phi_{\text {rel }}\right)$, and the position in MLT for the wave $\left(\varphi_{1}\right)$. The compressed background field is equivalent to a spatial oscillation of the field, imposed in the frame of the drifting particles. Absolute velocities of the particles are determined by MLT as per equation (2.1.27), here written as:

$$
v_{\phi}=\frac{\gamma_{m} \mu}{e L}\left(1+\frac{B_{c}}{B_{0} / L^{3}} \cos \phi\right)
$$

The presence of an azimuthal gradient in the magnetic field can also contribute an additional term to the electric field amplitude as a function of MLT, but for the purpose of demonstrating 
ideal wave-particle interactions we assume a constant amplitude independent of any spatial coordinate.
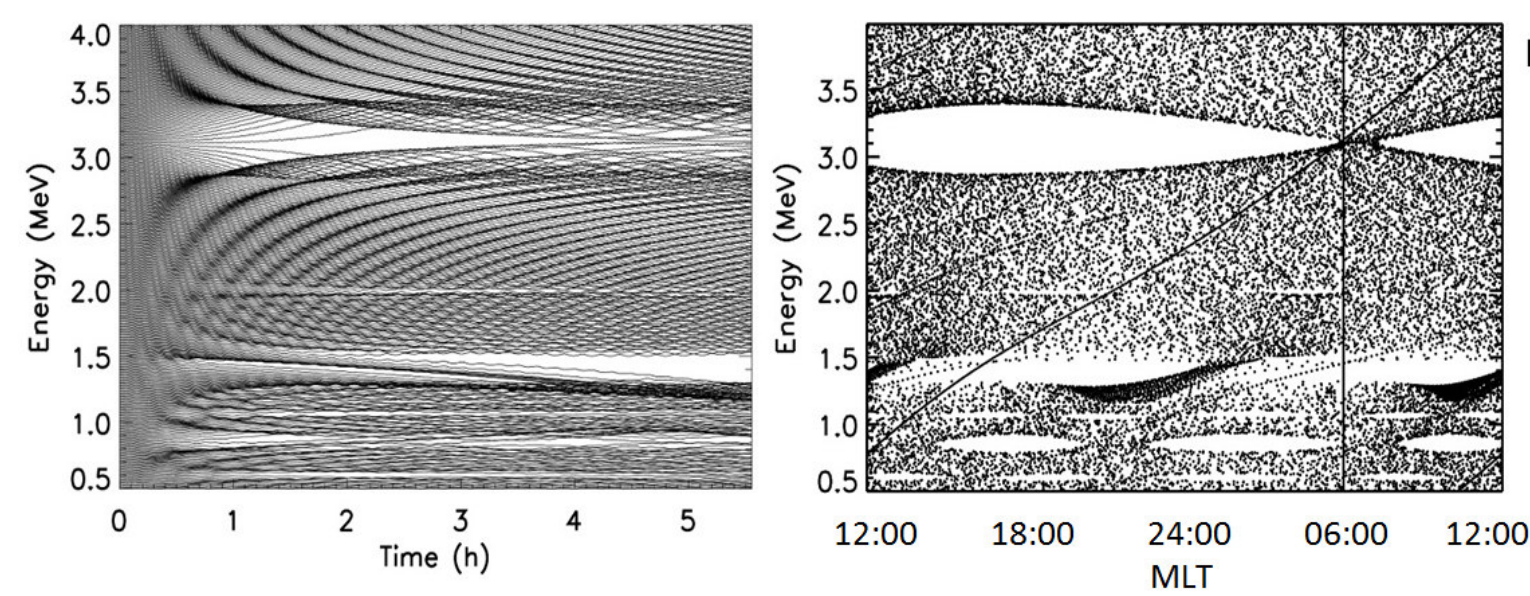
Harmonic: $1 s t$

2nd $3 r d$

Figure 4-5: Electron energy gain/loss due to interaction with a monochromatic wave electric field with $E_{L}$ component, assuming an azimuthally asymmetric field dipole. Left: Energy as function of time. Right: Poincare surface of section of electron phase space at $T_{s}=T_{w}$.

Similarly to the models and simulations in section 4-2, we consider electrons with starting location at dawn $\mathrm{MLT}_{0}=06: 00\left(\varphi_{0}=3 \pi / 2\right)$, at $\mathrm{L}_{0}=5.0$, and with uniform distribution in initial energy $\mathrm{W}_{0}=0.5-4.0 \mathrm{MeV}(\Delta \mathrm{W}=0.1 \mathrm{MeV})$. The drift frequencies range between $0.7-4.9 \mathrm{mHz}$ while the wave is set at a frequency of $\mathrm{f}_{\mathrm{w}}=3.1 \mathrm{mHz}$. Not only is the main harmonic resonant scattering visible, but also interactions with $1^{\text {st }}$ and $2^{\text {nd }}$-order subharmonics at lower energies for which the resonant condition is $n f_{d}=f_{0}$, where $n=2$ and $n=3$. The fundamental resonance for particles starting at about $3.1 \mathrm{MeV}$, corresponding to $n=1$, has the largest width compared to its subharmonics. The change in particle energy is due to a drift perpendicular to the Roederer L-shell described by equation (2.1.10). 


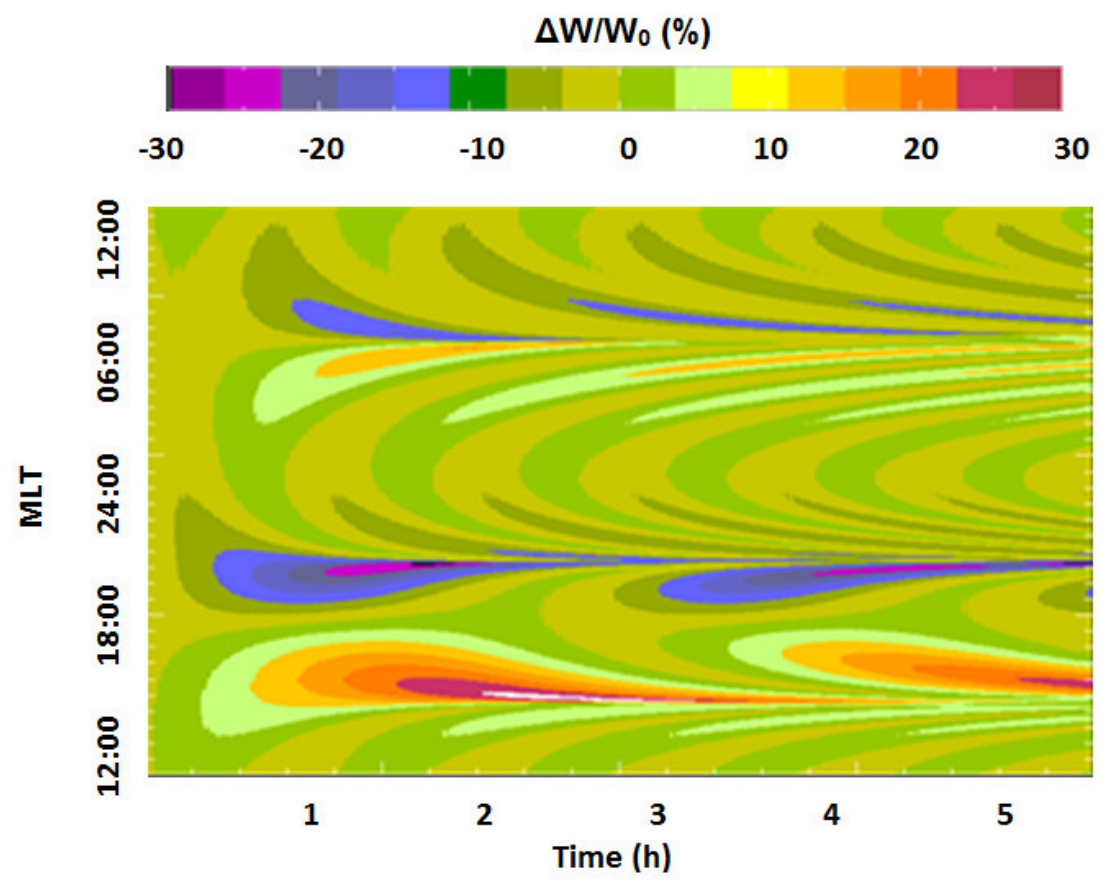

Figure 4-6: Electron energy change due to interaction with monochromatic wave electric field with $E_{L}$ component, assuming an azimuthally asymmetric magnetic field dipole. Electrons are launched at all MLTs.

Since $\mu$ is not only dependent on the initial perpendicular energy, but also the local magnetic field at the starting point, an initial distribution of particles along a range of L-shells would produce similar energy shift patterns as seen in the left-side graphs in figure 4-2. One way to do this is by placing electrons uniformly distributed in a concentric ring and equal perpendicular energies, $\mathrm{W}_{0}$, which gives a range of initial drift frequencies, $\mathrm{f}_{\mathrm{d} 0}$. We show one such simulation in figure 4-6. They also experience different initial phases of the global wave, thus the patterns emerging at dawn and dusk. The electrons that are initially placed in the dusk region $\left(\mathrm{MLT}_{0} \sim 12: 00-24: 00\right)$ undergo closed phase space trajectories, much like the example for $\phi=3 \pi / 2$ in section 4.2 . 


\subsection{Electron Interactions with a Wave Electric Field of Mixed Polarization.}

A step toward a more realistic system of wave-particle interaction is by including both $\mathrm{E}_{\mathrm{L}}$ and $\mathrm{E}_{\phi}$ components of a wave as both polarizations can cause adiabatic electron position and energy scattering. The $\mathrm{E}_{\mathrm{L}}$ component requires an asymmetric dipole field in order to have such effect on electrons, thus we will use $B_{c}=30 \mathrm{nT}$ in equation (4.2.2) and use MLT coordinates where the wave phase is $\phi=0$. This section will continue the assumption of an ideal monochromatic wave.

We set the values of the first adiabatic invariant, $\mu$, by initially placing the particles in a ring at a $\mathrm{L}_{0}=5$ with initial energy of $\mathrm{W}_{0}=3.67 \mathrm{MeV}$. The wave parameters are: $\mathrm{f}_{1}=3.94 \mathrm{mHz}$, and $\mathrm{m}=2$, while the wave amplitudes $\mathrm{E}_{\mathrm{L}, 0}$ and $\mathrm{E}_{\varphi, 0}$ are independent variables. A good way of evaluating the efficiency in each field component is to quantify the maximum energy shift of the electron population, as already discussed at the end of section 4-2. It should be noted that, as for all cases in this section, this is an adiabatic mechanism where electrons eventually converge back to their initial energies. We show two examples of energy extremes found during the course of the simulations for two different electric field amplitudes (figure 4-7a). Two maximum energy peaks, depicting the resonance islands of the fundamental frequency, emerge at about dusk and dawn in local time, with an azimuthal shift depending on whether it is a gain or loss. The shapes of these extreme values are related to what is observed in the dusk sector in figure 4-6. By dividing the initial MLT distributions of electrons between dawn (00:00-12:00 MLT) and dusk (12:00-24:00 MLT) sectors, the peaks from both regions can be quantified. We find the maximum energies as functions of the electric field component amplitudes and plot them in figure $4-7 \mathrm{~b}$. 


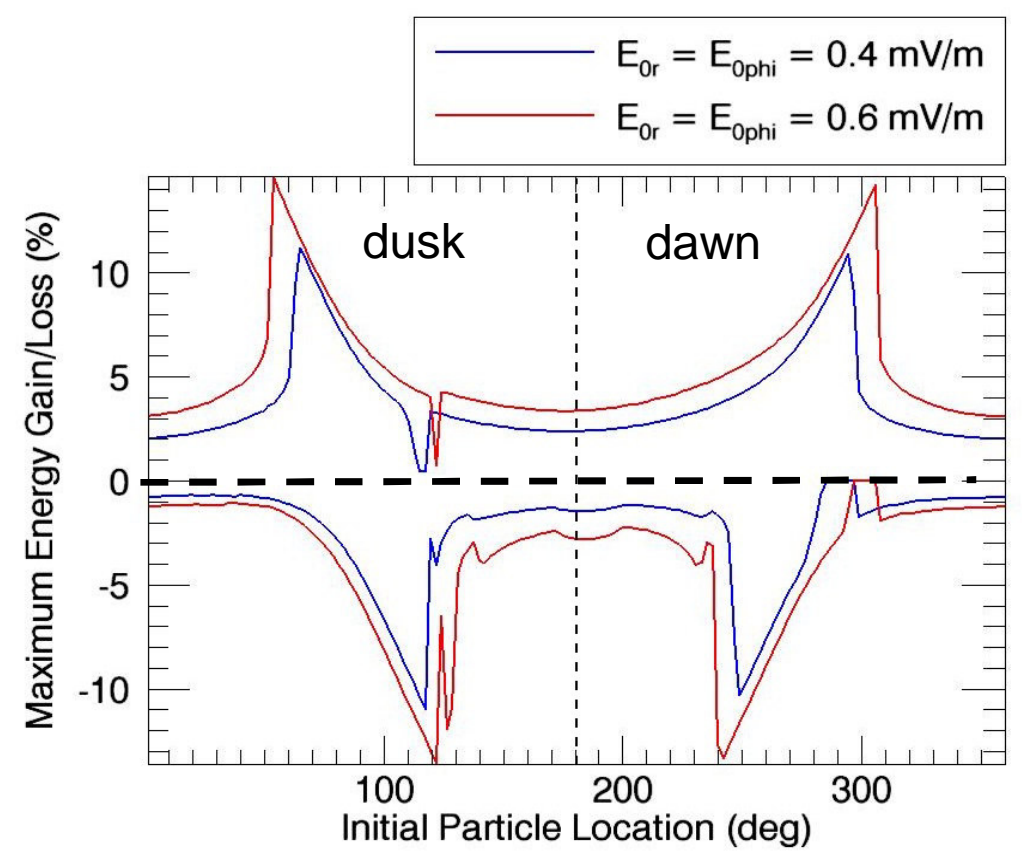

Figure 4-7a: Two cases of maximally gained or lost energy for all electrons, with a wave $E_{\phi}$ field in a compressed dipole field.

In addition to the fundamental mode, we examine $1^{\text {st }}$ order subharmonic interactions as well. This is done by initially placing the particles at dawn (06:00 MLT) at the same $\mathrm{L}_{0}$ and $\mathrm{W}_{0}$ as before, and then find the maximum energy gain or loss for each harmonic resonance. We separate each resonant island width, as these maximum energies represent, into the fundamental mode as well as the $1^{\text {st }}$ subharmonic mode and plot respective maximum energies in figure $4-7 c$. 


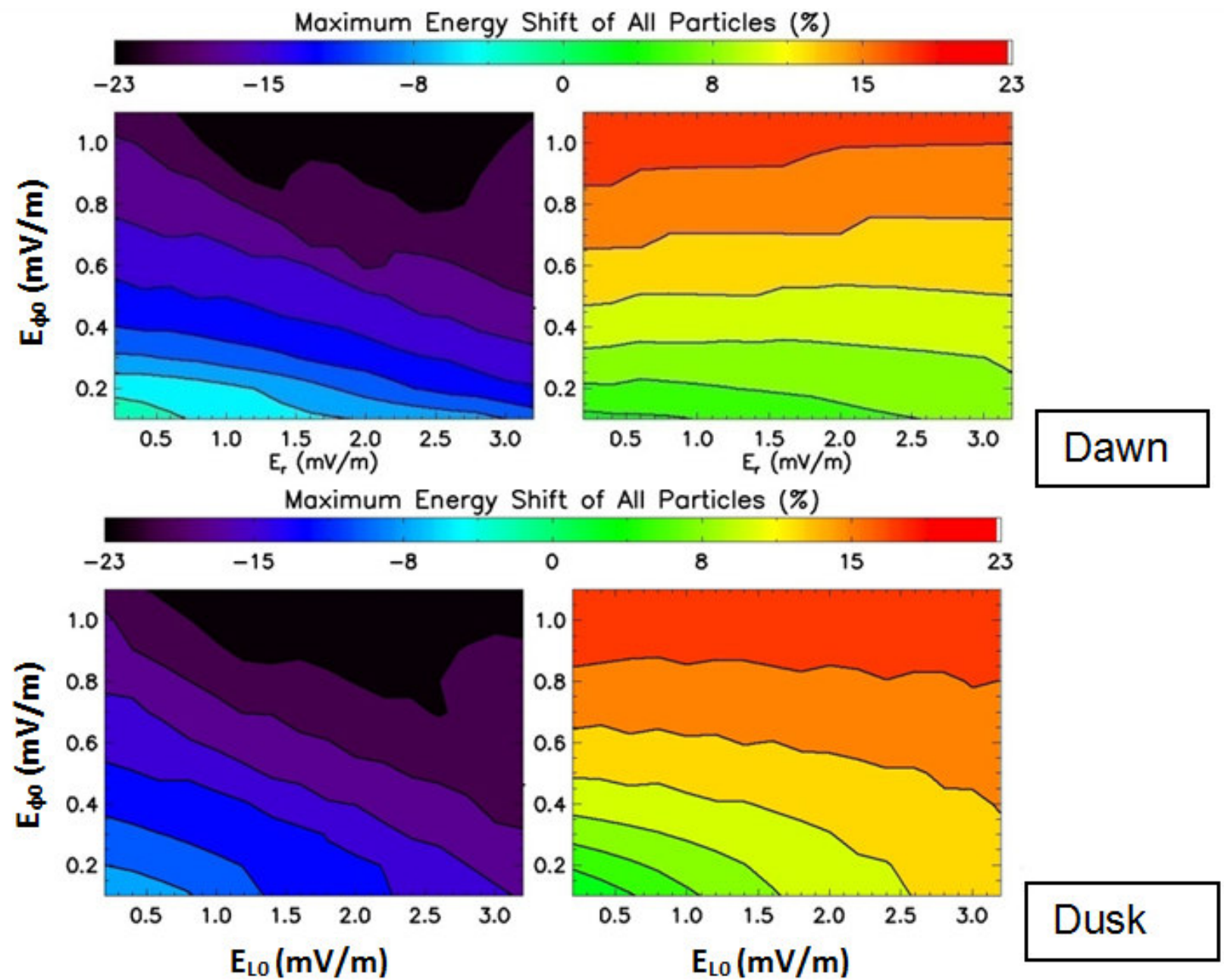

Figure 4-7b: Maximum energy gain or loss for electrons launched in either the dawn (00:00

- 12:00 MLT) or the dusk (12:00 - 24:00 MLT) sector in a compressed dipole field. The

electrons interact with wave electric fields with parametrized $E_{L}$ and $E_{\phi}$ components. 

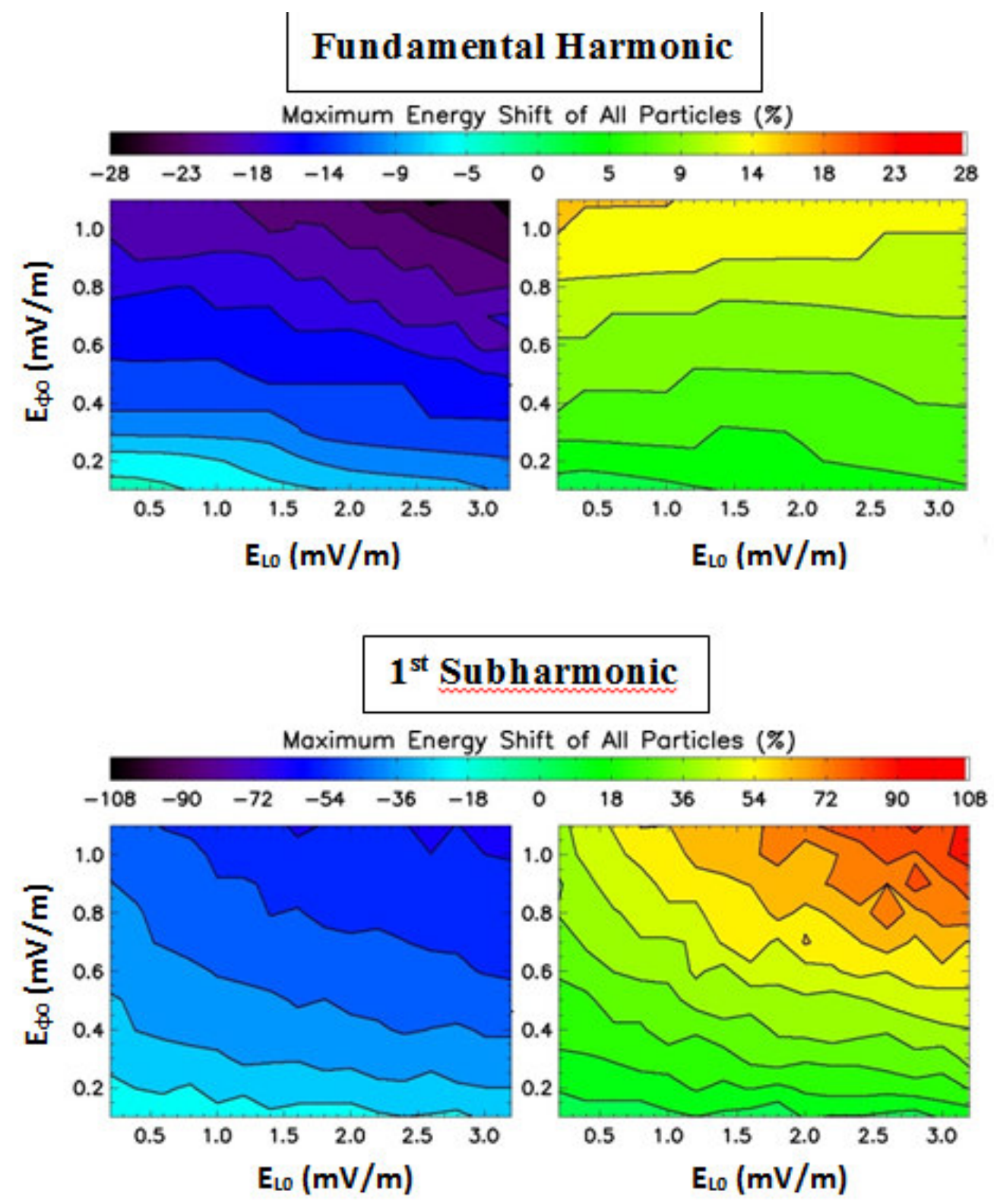

Figure 4-7c: Maximum energy gain or loss for electrons launched at dawn (06:00 MLT) at either fundamental frequencies or at $1^{\text {st }}$ subharmonic frequencies in a compressed dipole field. The electrons interact with wave electric fields with parametrized $E_{L}$ and $E_{\phi}$ components. 
Consistent with earlier studies ( Ukhorskiy et al., 2005 ) the $\mathrm{E}_{\phi}$ component is several times more effective in driving particle transport and energy scattering compared to the $\mathrm{E}_{\mathrm{L}}$ component. For example, electrons launched in the dusk sector and within the fundamental resonance (figure 4-6a, left plot), experience 3-5 times stronger energy gain or loss from the $\mathrm{E}_{\phi}$ component compared to the $\mathrm{E}_{\mathrm{L}}$ component for $0.1<\mathrm{E}_{\phi 0}<0.7 \mathrm{mV} / \mathrm{m}$. Electrons launched in the dawn section experience about 5-8 times stronger energy loss with the same comparison, while in energy gain the factor is $>10$. Since electrons launched at dusk would end up in closed phase space curves when driven by a wave $\mathrm{E}_{\mathrm{L}}$ field in this configuration, they also have twice as large $\Delta \mathrm{W}$ - this accounts for the factor of 2 difference between the dusk and dawn electrons. But even so, the dusk particles still scatter less due to a wave $E_{\phi}$ field compared to a wave $\mathrm{E}_{\mathrm{L}}$ field, where the only remaining factor is the magnetic field compression, $\mathrm{B}_{\mathrm{c}}$. From equation (2.13) the relation is $\Delta W \propto \sqrt{B_{c}} \quad$. To reach the factor of 3-5 difference between the $\mathrm{E}_{\phi}$ and $\mathrm{E}_{\mathrm{L}}$ modes of the same amplitude for this case where $\mathrm{B}_{\mathrm{c}}=$ $30 \mathrm{nT}$, the solar wind-compression amplitude would have to be adjusted to $\mathrm{B}_{\mathrm{c}}=180-450 \mathrm{nT}$, which are unrealistic values for Earth's magnetosphere.

The contributions from both wave electric field components are not entirely uncoupled; the $\mathrm{E}_{\mathrm{L}}$ component contributes to the azimuthal drift velocity of electrons, leading to prolonged or shortened exposure to total wave field power and, consequently, increased or decreased radial drift velocity. For example, as seen in the contour plot in figure $4-7 b$, the $E_{L}$ component provides a significant contribution to electron energy gain if the particles are launched at dusk and at fundamental resonant drift frequencies, and if the wave field has a weak $\mathrm{E}_{\phi}$ amplitude. 
Another example (fig 4-7c), is where electrons are launched at dawn, at subharmonic frequencies, and the wave field has a strong $\mathrm{E}_{\phi}$ amplitude. Regardless, the wave $\mathrm{E}_{\mathrm{L}}$ fields can be neglected for most scenarios in outer electron-belt dynamics.

\subsection{Electron Interactions with Two Coexisting $\mathbf{E}_{\phi}$ Waves}

This section will go back to the second case described in chapter 4.2 for a symmetric dipole, but with one additional wave added. Our interest is to understand particle transport when multiple phase space islands intersect with each other. We define a $2^{\text {nd }}$ wave at a frequency close to the $1^{\text {st }}$ one, with a spacing of $\Delta \mathrm{f}=\Delta \omega / 2 \pi=\mathrm{f}_{2}-\mathrm{f}_{1}$. Similar to the case for a wave in an asymmetric dipole in 4.1 there are two different phases and the initial location of the particles that must be taken into account, resulting in three free parameters. Clearly if one expands to higher number of waves the number of free parameters will increase as well. For example, three waves with unique phases and one azimuthal starting location for particles will yield the following combined parameters: $\phi_{1^{-}} \phi_{2}, \phi_{1^{-}} \phi_{3}, \phi_{1^{-}} \phi_{4}, \phi_{2^{-}} \phi_{3}, \phi_{2^{-}} \phi_{4}$ and $\phi_{3^{-}} \phi_{4}$. In other words, $\mathrm{N}_{\mathrm{k}}$ numbers of waves for $\mathrm{k}=>3$ yield $\mathrm{N}_{\mathrm{K}}$ ! parameter combinations, thus the problem quickly becomes unmanageable.

In one such simulation the particles are initially at $\mathrm{L}_{0}=5$ and magnetic local noon $\left(\varphi_{0}=0^{\circ}\right)$. The frequency of the first wave to be used in the simulations is set at $\mathrm{f}_{1}=1.87 \mathrm{mHz}$, while four values are used for $\mathrm{f}_{2}$. Those are: $2.02,2.07,2.12$ and $2.15 \mathrm{mHz}$ which correspond to 1.69, $1.74,1.79$ and $1.84 \mathrm{MeV}$ for resonant electron energies at the initial L-shell. The 
amplitudes of the waves are equal: $\mathrm{E}_{\phi, 1}=\mathrm{E}_{\phi, 2}=0.3 \mathrm{mV} / \mathrm{m}$, although the phase space resonance width does differ slightly due to the frequency of the second wave.

We show one case in figure 4-8 with a set of phases for two waves; $\phi_{0}=0$ and $\phi_{1}=\pi$. The frequency separation between the waves is $\Delta \mathrm{f}=0.15 \mathrm{mHz}$. For the one wave case, having a phase of $\phi_{0}=\pi / 2$ causes the electrons to propagate along open phase space curves, while $\phi_{0}=$ $3 \pi / 2$ places the electrons on a closed trajectory in phase space.
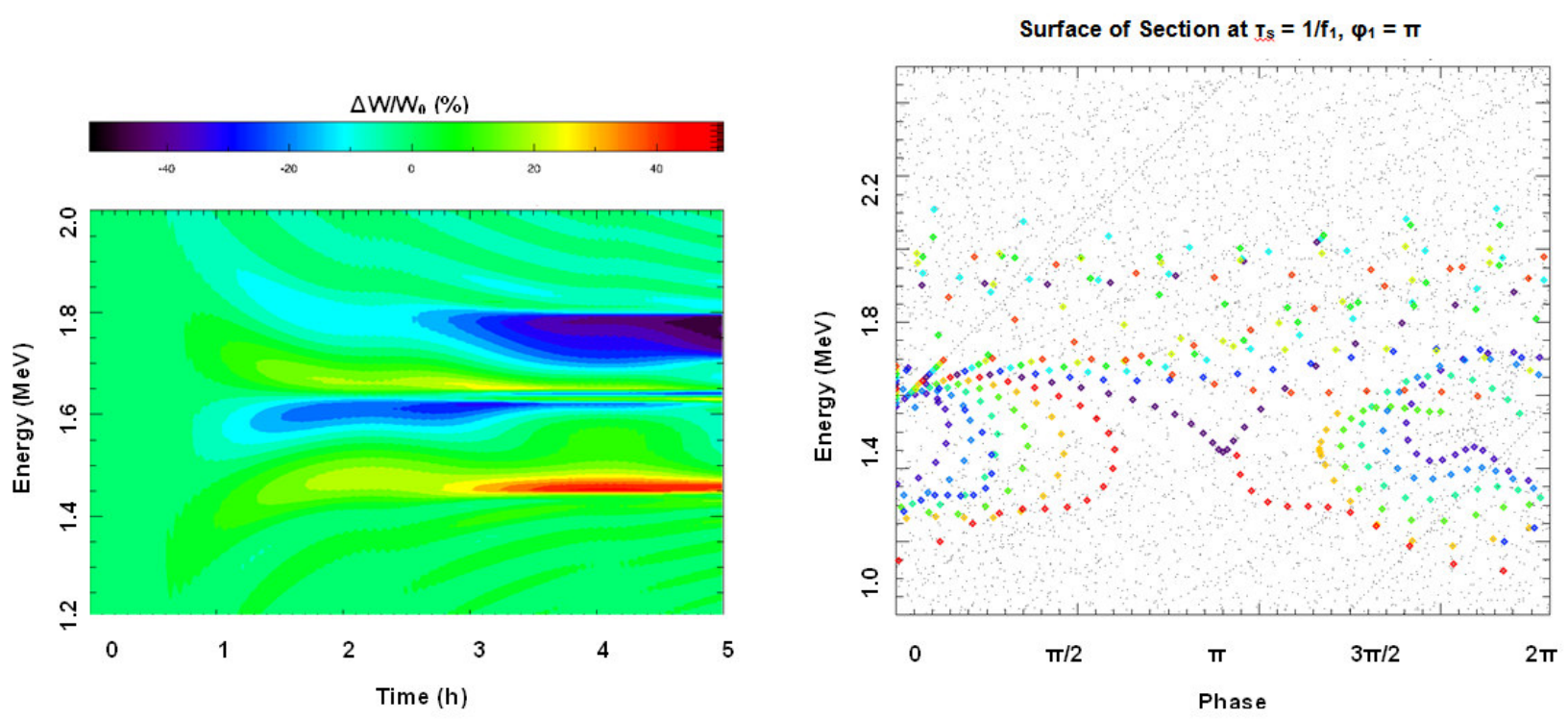

Figure 4-8: Electron energy scattering due to two coexisting $E_{\phi}$ waves. The electrons are launched at midnight (00:00 MLT) in a symmetric dipole. Wave 1 is at $f_{0}=1.87 \mathrm{mHz}$ and the relative wave-particle phase $\phi_{2}=0$, while wave 2 has the following frequency $f_{1}=2.02 \mathrm{mHz}$.

Similar to the single wave case, the pattern is qualitative different depending on $\phi_{1}$. The acceleration here is global since the relative phase $\varphi_{2}$ covers all azimuthal coordinates. We average all energies for all $\varphi_{2}$ (fig. 4-9 top), as well as for all $\varphi_{2}$ and energies within the resonant band, $\mathrm{W}_{0}=1.4-1.8 \mathrm{MeV}$ (fig. 4-9 bottom). For these averages we investigate the simulations for $\Delta \mathrm{f}=0.05 \mathrm{mHz}$ that have the strongest resonance overlap. 

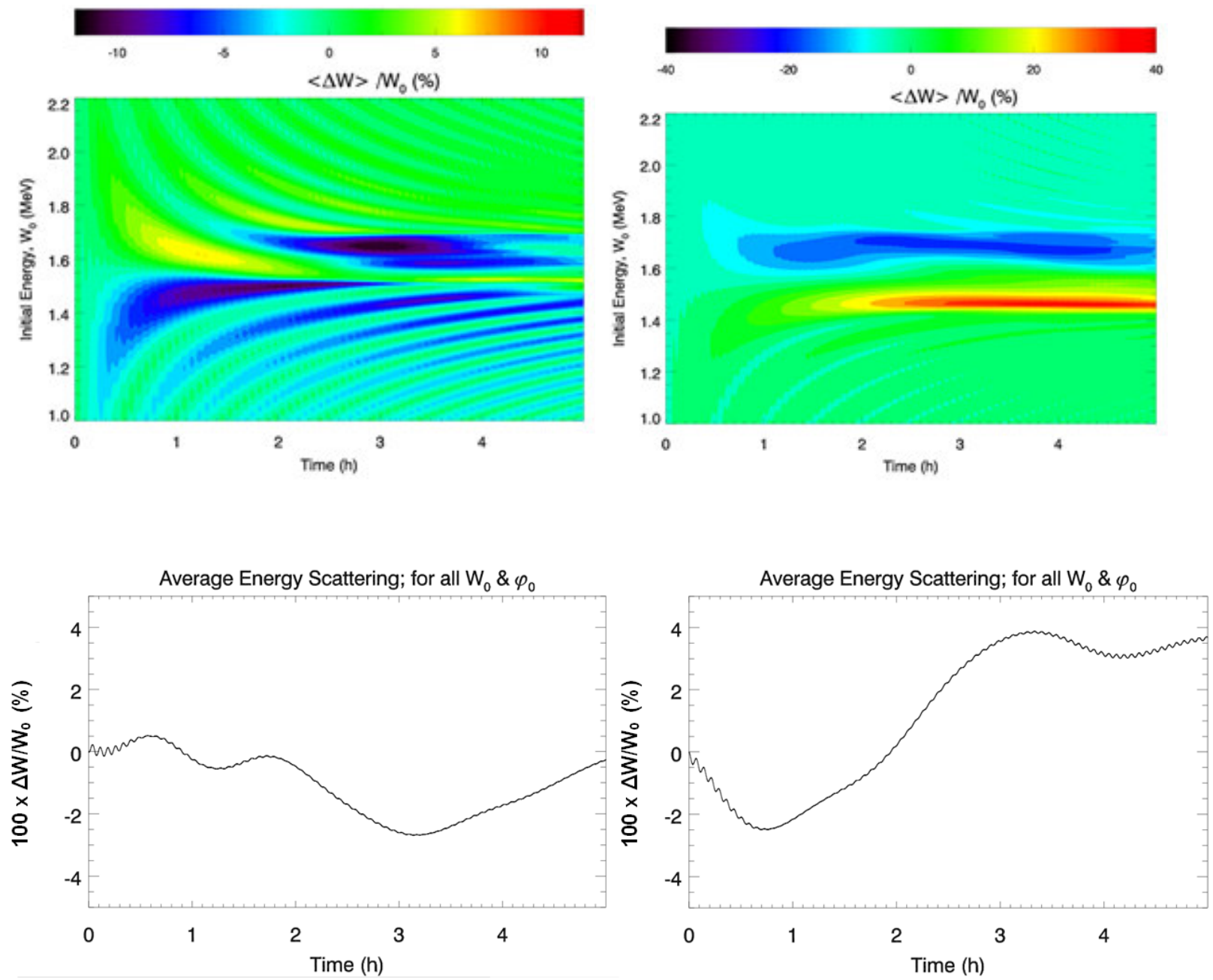

Figure 4-9: Averaged energy over: top) all initial phases $\varphi_{2}$, bottom) all initial phases $\varphi_{2}$ and energies $W_{0}=1.4-1.8 \mathrm{MeV}$

Even after the energy has been averaged for each $\varphi_{2}$ coordinate the outcome is different depending on $\phi_{1}$. Thus global acceleration can be affected due to the choice of phase in one of the waves in a 2 -wave system. For $\phi_{1}=\pi / 2$ the net energy shift is negative with a minimum at $\max \left(\left\langle\Delta_{-} W\right\rangle_{\phi_{2}}\right) / W_{0} \approx-12 \%$ and averaged over the resonance energies: $\max \left(\left\langle\Delta_{-} W\right\rangle_{W_{0}, \phi_{2}}\right) / W_{0} \approx-3 \%$, thus there is a small loss of energy for a large fraction of the electron population. For $\phi_{1}=3 \pi / 2$ the energy averaged over $\varphi_{2}$ can gain up to $40 \%$ 
additionally to $\mathrm{W}_{0}$, while losing a maximum of $-19 \%$. The average over both $\mathrm{W}_{0}$ and $\varphi_{2}$ has an initial dip into $-2.5 \%$, but after $\sim 2$ hours increases up to $+4.0 \%$, which are small numbers compared to the maxima. Thus both the global and local electron populations can experience many different energy shift patterns when interacting with waves that have overlapping resonance widths. The process is still adiabatic, meaning that there still is a nonlinear recurrence period, although it is relatively long. Within an hour of simulation time electrons begin scatter significantly, and do not revert back to their initial energies again for at least the next 4 hours, which is a large difference compared to the single wave case where the energies remain shifted for only about 1 hour (fig. 4-3 bottom).

For further analysis, we use the drift frequency as the main parameter since it represents both the radial drift as well as the subsequent change in kinetic energy. We show four cases of maximum drift frequency shift in figure 4-9 for each initial condition. The frequency separations of the two waves are set to $\Delta \mathrm{f}=0.15$ and $0.20 \mathrm{mHz}$ which would make $K \geq 1$ in equation (2.2.14).

In these plots, by using the relative change in frequency as in $f_{0}+\Delta f$, particle transport across both wave frequencies can be established by comparing the peaks to a horizontal line; should this occur for an electron in resonance with wave \#2 the negative peak will end up beyond the frequency of wave \#1, projected onto the diagonal line. The simulations run for at least one period of the nonlinear oscillation ( see Eq. (2.2.9) ) in order to cover potential maxima, i.e. $\mathrm{T}_{1-2} \sim 6$ hours. Figure 4-10 is a reference plot where only one monochromatic wave at $\mathrm{f}=$ $1.87 \mathrm{mHz}$ has been used for two different phases $(\phi=\pi / 2,3 \pi / 2)$. We construct similar reference plots for each monochromatic wave separately in figure 4-11, but in frequency shift instead. In the same picture we also plot the maximum shifts in drift frequencies for electrons 
when both waves coexist in the system. Here follows a description of 2-wave-particle interactions for the two phase combinations we have assumed. The denotation of "open" and "closed" phase space (PS) trajectories indicate electrons that would follow one or the other should there only exist one wave in the system.

Case A: Open phase space trajectories intersect each other here, due to equal phases of both waves at $\phi_{1}=\phi_{2}=\pi / 2$. It shows a clear case for Chirikov's criterion being in effect as the drift frequency of electrons shift across both phase space islands caused by both waves. This occurs for the spacing of $\Delta \mathrm{f}=0.2 \mathrm{mHz}$ between the waves. The drop in frequency is more powerful than for the increase, due to the radial asymmetry in scattering.

Case B: Open and closed phase space curves intersect in this phase space diagram. The drift frequency decrease initially caused by wave \#2 is sufficiently large to reach the maximal extent of drift frequency that can be potentially caused via resonant effect by wave \#1. The interesting detail here however is that the extent of the frequency shift wave \#2 alone, as shown for the reference plot, can only reach a lowest point at $f_{d, f}=1.93 \mathrm{mHz}$, while the resonant frequency for wave $\# 1$ is at $f_{1}=1.87 \mathrm{mHz}$, thus there is no resonance overlap. Therefore the separatrix in phase space for wave \#1 has been broken and electrons are able to pass across it. There is a narrow peak at $\sim 1.90 \mathrm{mHz}$ which hints of the secondary step caused by wave \#2. In figure 4-12 it is apparent that this feature is indeed a perturbation by this second wave.

Case C: The previous case is repeated here, but with a slightly more narrow separation of the two waves $(\Delta \mathrm{f}=0.15 \mathrm{mHz})$. The effect is concentrated on whether electrons following open phase space trajectories can break away and enter the circularly, closed pattern by wave \#2. 
The resonant width of wave \#1 is smaller than for wave \#2, so that it can never overlap in resonance without being engulfed by the extent of the wider resonant band above it. It does appear to widen the peak of electron frequencies that undergo resonant transport, but there is no additional enhancement in its magnitude as would be expected because of the total frequency engulfment.

Case D: Likewise as in case A, two waves of the same phases are used again, this time $\phi_{1}=\phi_{2}$ $=3 \pi / 2$, each wave creates a closed phase space pattern. A sharp negative peak for a single electron resonant with wave \#2 appears. Since it is aligned horizontally with the resonant peak of wave \#1, this electron will eventually end up experiencing transport across both resonances and lose energy as well as drift frequency.

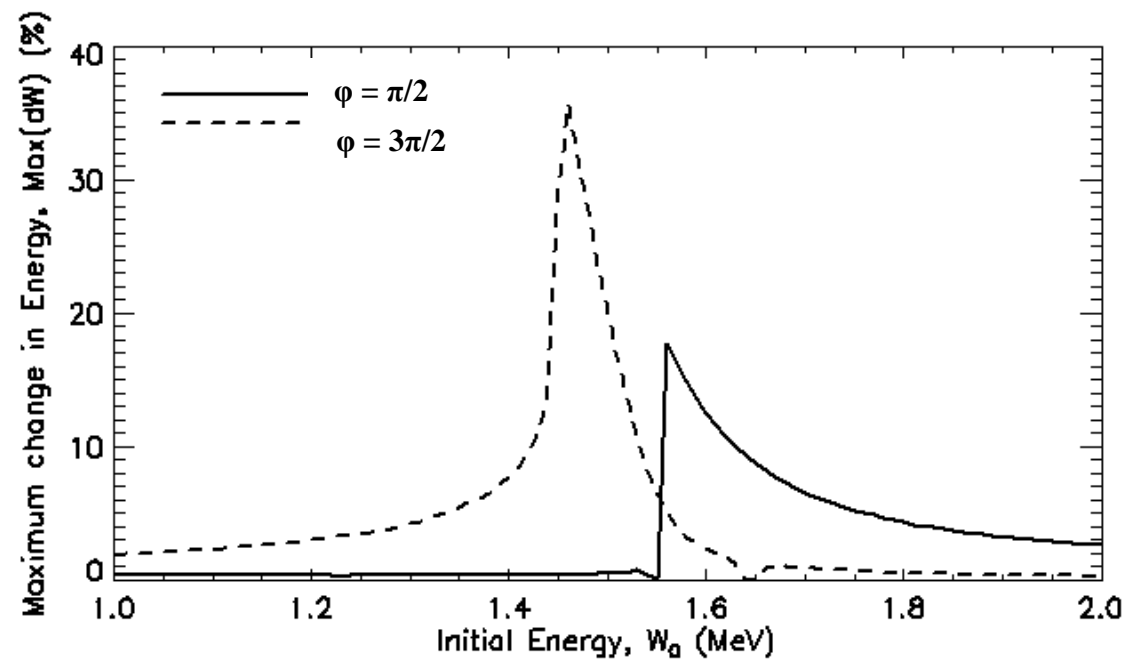

Figure 4-10: Maximum energy gain of electrons due to a monochromatic wave $E_{\phi}$ field.

As stated, the positive-negative asymmetry between frequency gain and loss yields a stronger negative $\Delta f_{d}$ than a positive shift. This is especially apparent in figure 4-11a, but also 4-11d. 
Since the negative side of the resonant band is wider it is able to overlap with a wave at a lower frequency at less frequency separation than for the opposite.

Now we expand these four cases to include a distribution of phases for wave \#2. Each phase is equivalent to placing the electrons at particular MLTs, like the case for $\mathrm{B}_{\mathrm{c}} \neq 0$, thus it can be regarded as global transport of particles. The frequency separation is also expanded to encompass $\Delta \mathrm{f}=0.05,0.10,0.15,0.20,0.25$ and $0.30 \mathrm{mHz}$. Thus for each phase and frequency separation used, we run a simulation with the same number of electrons with distributed energies (= drift frequencies).

When we examine the maximal negative shift in drift frequencies in figures $4-12$, we see different outcomes due to the choice of phases for the two waves. There are concentrated ranges of frequencies and phases for which electrons at and above $f_{2}$ are able to shift to frequencies below $f_{1}$. Two-resonance transport appears to be more effective for $\pi<\phi_{2}<2 \pi$ than for lower phase values. For frequency separations at $\Delta \mathrm{f}=0.25$ and $0.30 \mathrm{mHz}$ the multiresonance transport ceases for all electrons, although some perturbations from wave \#1 can still occur. 

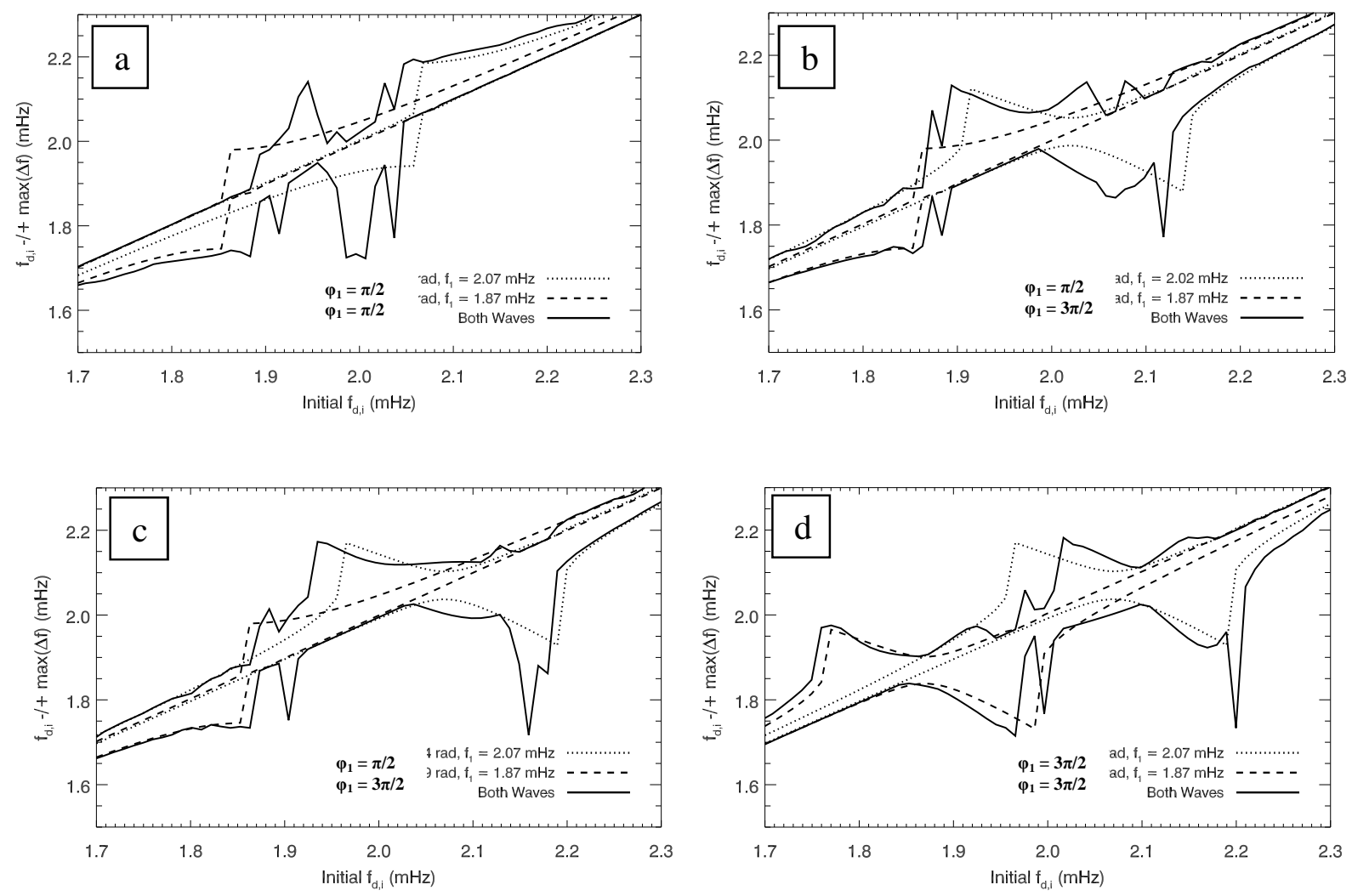

Figure 4-11: a) Open PS trajectory intersects with another open PS trajectory. b) Open PS trajectory intersects with a closed PS trajectory. c) Closed PS trajectory intersects with an open PS trajectory. d) Closed PS trajectory intersects with another closed PS trajectory. 

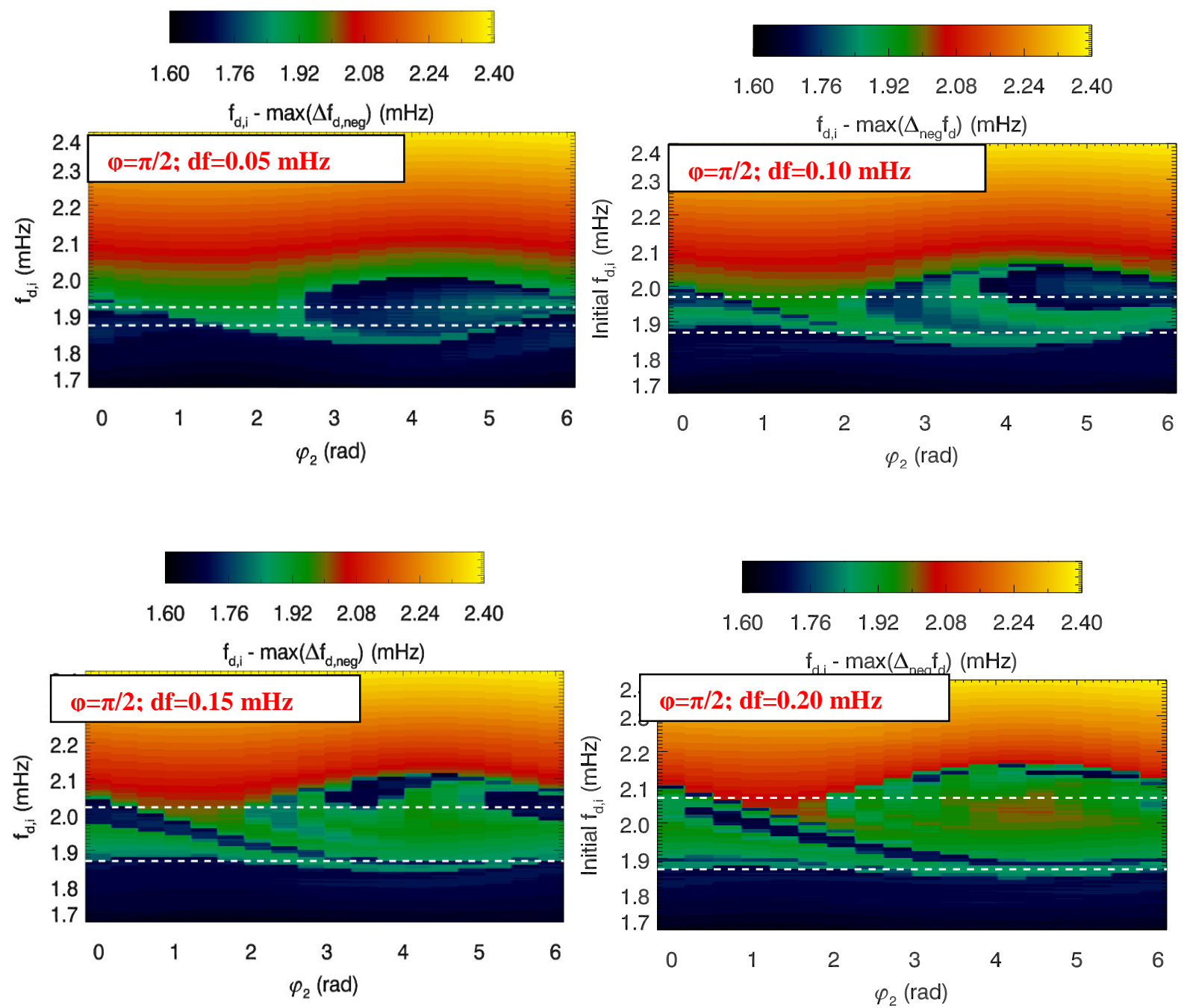

Figure 4-12a: Maximum negative drift frequency shift for electrons interacting with two monochromatic wave $E_{\phi}$ fields. The phases are $\varphi_{1}=\pi / 2$, and $\varphi_{2}=0-2 \pi$ for each simulation. 

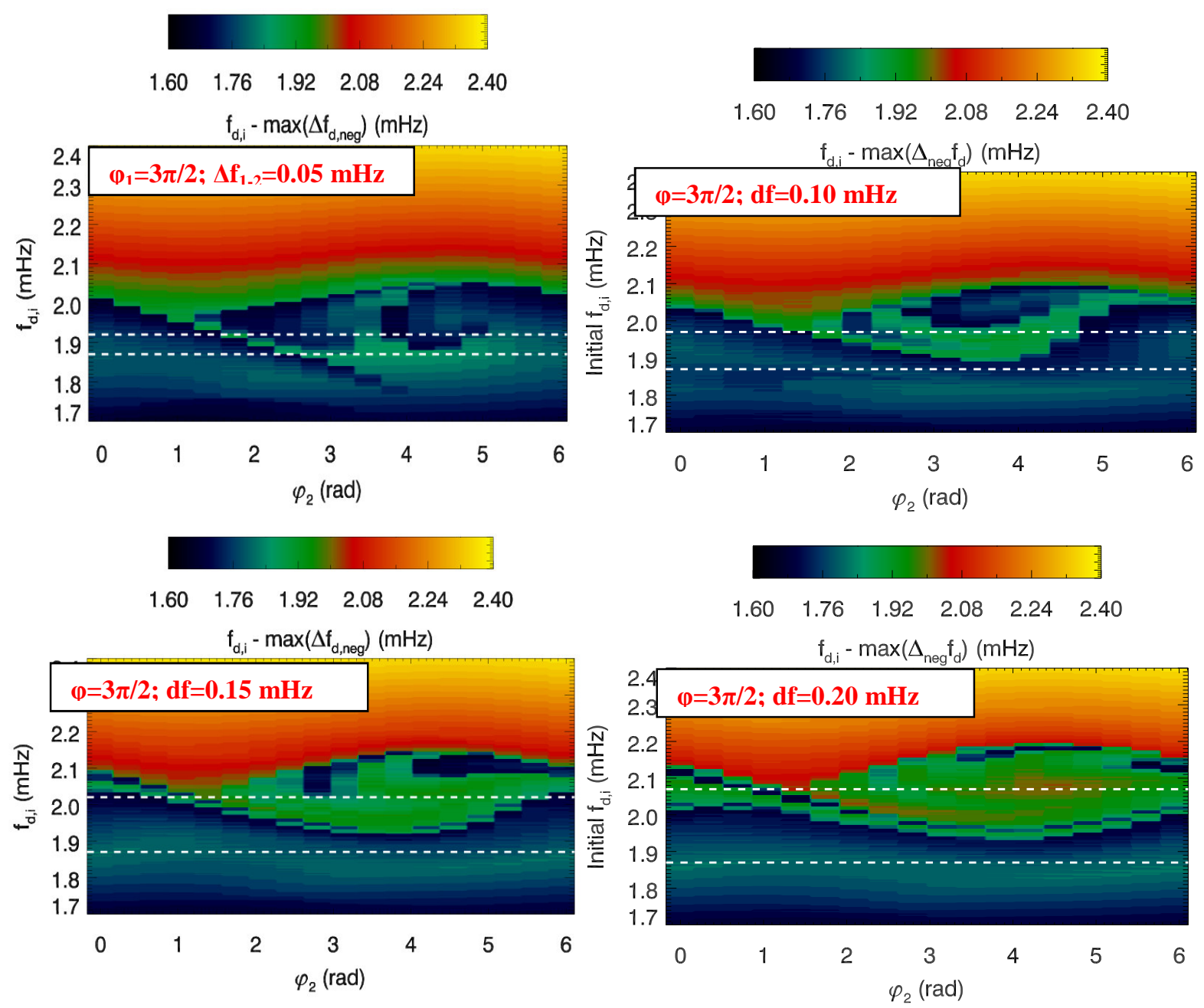

Figure 4-12: Maximum negative drift frequency shift for electrons interacting with two monochromatic wave $E_{\phi}$ fields. The phases are $\varphi_{1}=\pi / 2$ or $3 \pi / 2$, and $\varphi_{2}=0-2 \pi$ for each simulation. 
We calculate the stochasticity parameter K ( Eq. (2.2.14) ) by using reference simulations where only one wave was used at each $\mathrm{f}_{2}$ discussed in this section. There are clear phase space islands in each simulation of which the frequency width can be extracted via

$$
\Delta f_{\text {island }}=\left|\Delta f_{d, \text { max }}\right|+\left|\Delta f_{d, \text { min }}\right|
$$

Then $\mathrm{K}$ becomes:

$$
K=\left(\frac{\Delta f_{\text {island }}}{\Delta f_{1-2}}\right)^{2}
$$

The parameter $\mathrm{K}$ is independent of the phases of the waves and only comprises of the maximum drift frequency change of the particle in both positive and negative direction combined due to a single wave, neglecting any kind of perturbation.

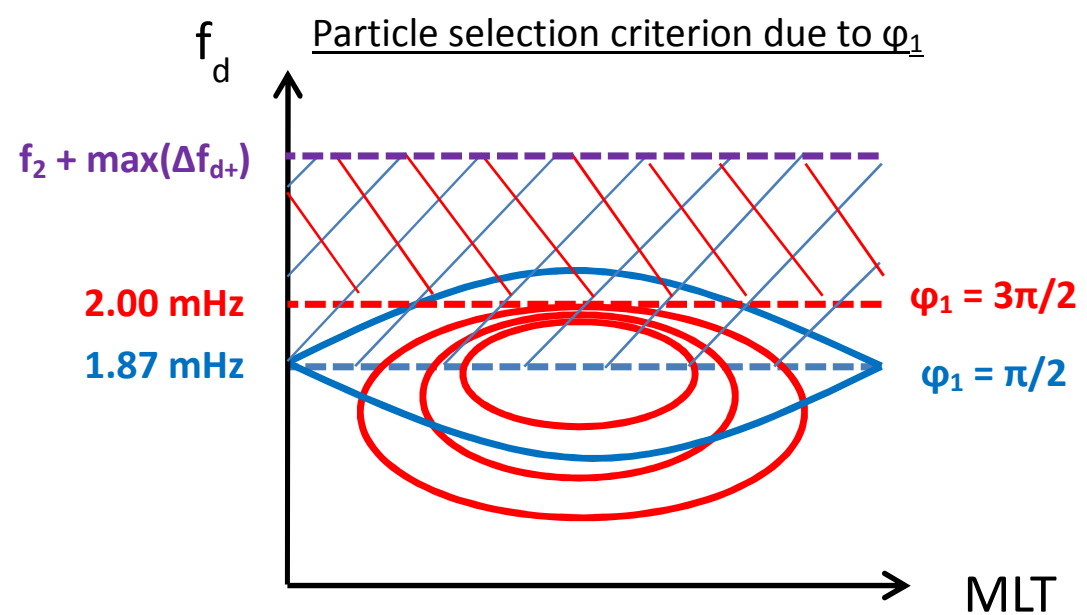

Figure 4-13: Particle selection criterion for verifying resonance transport in a 2-wave system. 
We make an estimate in the effectiveness of 2-resonance transport by finding the numbers of electrons that at some point throughout each simulation undergo sufficient negative shift in drift frequency to make such transport possible. The criterion for selecting electrons is that drift frequencies must be above the separatrix of wave $\# 1$, i.e. $\mathrm{f}_{\mathrm{d}}>1.87 \mathrm{mHz}$ for $\phi_{1}=\pi / 2$, and $\mathrm{f}_{\mathrm{d}}>2.00 \mathrm{mHz}$ for $\phi_{1}=3 \pi / 2$. The upper limit is at $\mathrm{f}_{\mathrm{d}}<\mathrm{f}_{2}+\max \left(\Delta \mathrm{f}_{\mathrm{d}+}\right)$ in order to remain within the resonant bandwidth of wave \#2. This drift frequency criterion is illustrated in figure 4-13 where the phase space trajectories caused by wave \#1 alone are included. We normalize the counted particles into a fraction percentage of the total number of particles within this frequency range. Both the frequency spacing between the waves as well as the overlap parameter $\mathrm{K}$ are shown on the $\mathrm{x}$-axis, while the uncertainty in $\mathrm{K}$ is too small to be displayed in this diagram ( error $\left.<1 \%: \Delta \mathrm{f}_{\mathrm{d}, \max }=0.112 \mathrm{mHz}+-0.010 \mathrm{mHz}\right)$. 


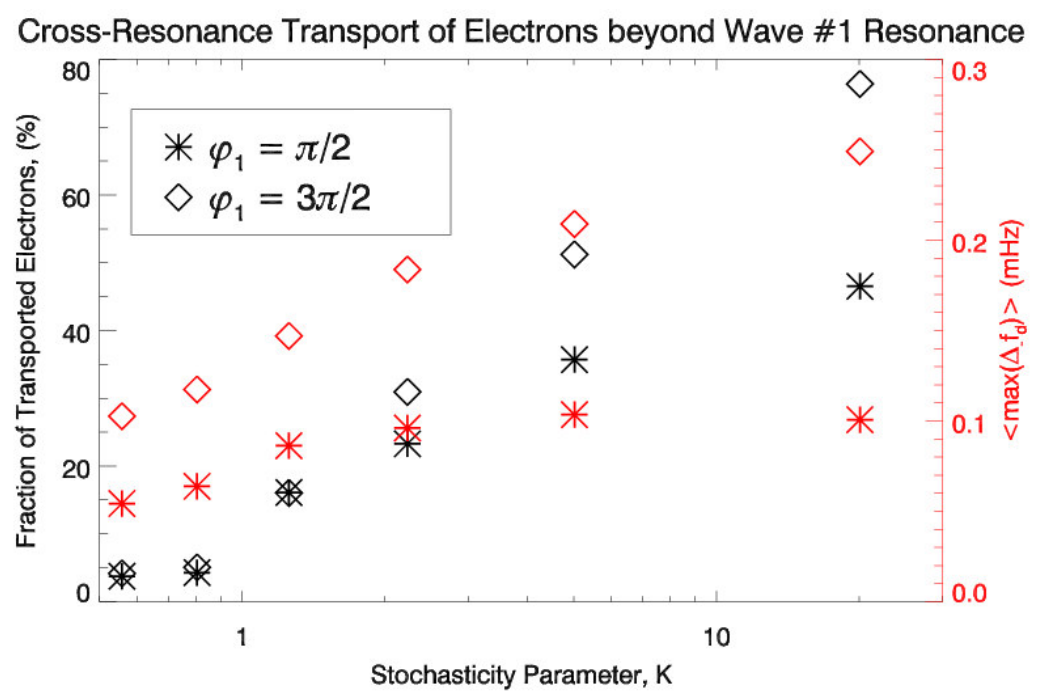

Figure 4-14: Black graph: Fraction of electrons undergoing transport to $f_{d 0}-\max \left(f_{d}\right)<f_{1}$ (1.87 mHz). Red graph: average maximal decrease in drift frequency for all electrons. All selected electrons are initially in resonance with wave \#2 and beyond the separatrix of wave \#1.

There are no electrons undergoing 2-resonant transport for both phases used in wave \#1 at the data points for $\mathrm{K}=0.56$ and $\mathrm{K}=0.80$. The values at those data points can be regarded as error margins in electron transport. The Chirikov criterion, as discussed in chapter 2-2, is satisfied for $\mathrm{K}>1.03 \pm 0.26$ when there is an increase in both the fraction of electrons and the average maximum drift frequency loss $\left\langle\Delta \mathrm{f}_{\mathrm{d} \text {,max }}\right\rangle$, thus verifying this fundamental threshold of chaotic motion. Beyond this onset for less separated wave frequencies, i.e. decreased $\Delta \mathrm{f}_{1-2}$, the number of electrons as well as $\left\langle\Delta \mathrm{f}_{\mathrm{d} \text {,max }}\right\rangle$ increase with $\mathrm{K}$. To quantify the result better we produce best fit power laws to each parameter, $\left\langle\Delta \mathrm{f}_{\mathrm{d} \text {,max }}\right\rangle$ and $\mathrm{N}_{2 \text {-res }} / \mathrm{N}_{\text {tot }}$, and only select the data points for which $\mathrm{K}>1$ : 


$$
\begin{gathered}
\phi_{1}=\pi / 2 \rightarrow<\Delta \mathrm{f}_{\mathrm{d}, \max }>\alpha \Delta \mathrm{f}^{0.43+-0.09} \\
\mathrm{~N}_{2-\text { res }} / \mathrm{N}_{\text {tot }} \alpha \Delta \mathrm{f}^{2.11+-0.24} \\
\phi_{1}=3 \pi / 2 \rightarrow<\max (\Delta \mathrm{fd})>\alpha \Delta \mathrm{f}^{0.68+-0.03} \\
\mathrm{~N}_{2-\text { res }} / \mathrm{N}_{\text {tot }} \alpha \Delta \mathrm{f}^{2.45+-0.08}
\end{gathered}
$$

It is clear from both fitted parameters that the efficiency in 2-wave-resonant transport is higher for $\phi_{1}=3 \pi / 2$. The improvement is about $58 \%$ steeper for the number of electrons transported and $16 \%$ steeper in $\left\langle\Delta \mathrm{f}_{\mathrm{d} \text {, max }}\right\rangle$ compared to $\phi_{1}=\pi / 2$. The difference in 2-resonance transport for the selected phases can in fact be visualized as an overlap of separatrices, which can be divided into an upper and a lower part of the separatrix with respect to the wave frequency. The lower separatrix of wave \#2, indepdendent of its phase $\phi_{2}$, must intersect with either an upper separatrix of wave \#1 with the phase $\phi_{1}=3 \pi / 2$, or the lower separatrix for $\phi_{1}=\pi / 2$, in order to allow 2-resonance transport. This is the reason why fewer electrons tend to undergo 2-resonance transport when $\phi_{1}=\pi / 2$.

The purpose of the study in this section was to demonstrate 2-wave-resonance transport of particles. One limitation is the lack of temporal information in the maxima method that has been used here. As shown in the time evolution plots and the recurrence rates, it can take hours for a particle to undergo a complete transfer across a second resonance. It may also not remain at its extreme energy for all time and will eventually end up reverting back toward its original state. 
Not only is the timing issue not fully explored here, but there still is the challenge of introducing more than two waves by which the complexity increases rapidly. The more waves added to the system the more the particle trajectories become increasingly chaotic and complex, thus different means of finding new energies and locations for them must be employed. It only becomes possible to establish a more straightforward analytical determination of particle scattrering through diffusion theory. In fact, as mentioned before the overlap parameter $\mathrm{K}$ is also known as the stochasticity parameter [ Lichtenberg \& Lieberman, 1992 ] since it is used to quantify the degree of stochasticity in the system. If many waves are involved it means that for $\mathrm{K}>1$ the transport can be considered a diffusive process for sufficiently long time scales. This scenario will be a main motivation for chapters 5 and 6 .

\subsection{Electron Interactions with an $\mathrm{E}_{\phi}$ Wave in an MLT Sector}

All waves used in the previous discussions of this chapter have been assumed to be distributed globally, i.e. over the entire MLT range. In this section a description of wave-particle interaction is given where the wave is confined in an MLT sector. Returning to the discussion in chapter 3 we understand that ULF waves typically become generated and propagate locally in geomagnetic space. Figure 3-3 reveals concentrated coordinates where the occurrence of wave electric fields is high - especially in the dayside sector. 
In this dissertation we will always assume the wave field amplitudes to be constant, independent of radial distance, and thus the wave amplitudes are uniform at all coordinates within the MLT sector. In this section we assume $B_{c}=0 \mathrm{nT}$.

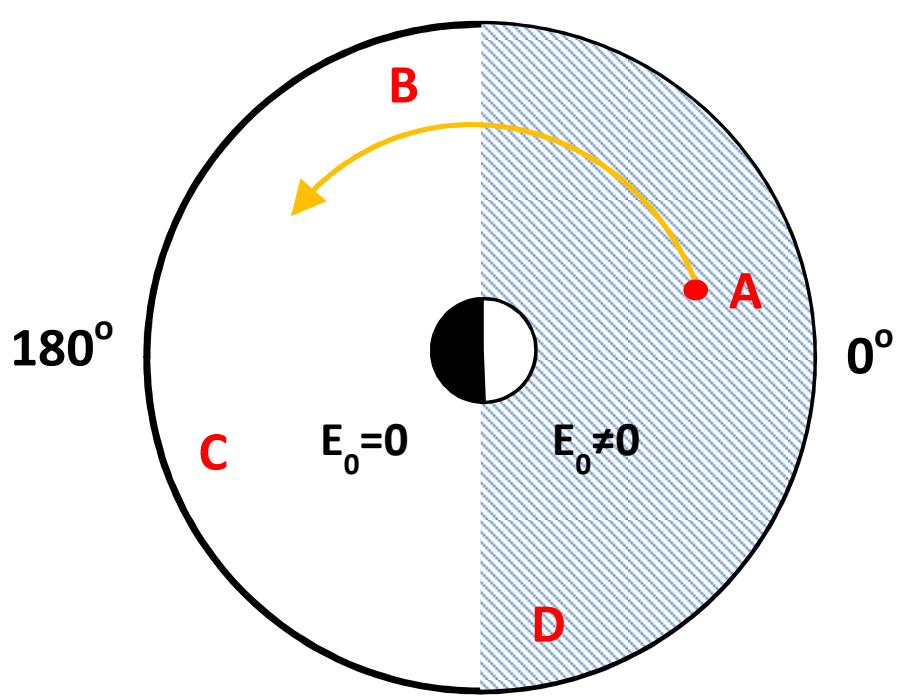

Figure 4-15: Schematic of MLT sector

with strong ULF wave activity.

A new set of simulations are shown in a similar manner as earlier examples in this chapter, in a series of figures (4-16). We test two wavenumbers: $\mathrm{m}=0$ and $\mathrm{m}=2$. For the non-zero wavenumber the endpoints of the wave are defined by with the spatial boundaries of the sector. The initial conditions of the electrons are $\mathrm{L}_{0}=6.6, \mathrm{~W}_{0}=1.2-2.0 \mathrm{MeV}$ and $\phi_{0}=0-24 \mathrm{~h}$, which ensures distributed $\mu$ values. 


$$
\mathrm{m}=0(\mathbf{f}=2.5 \mathrm{mHz})
$$
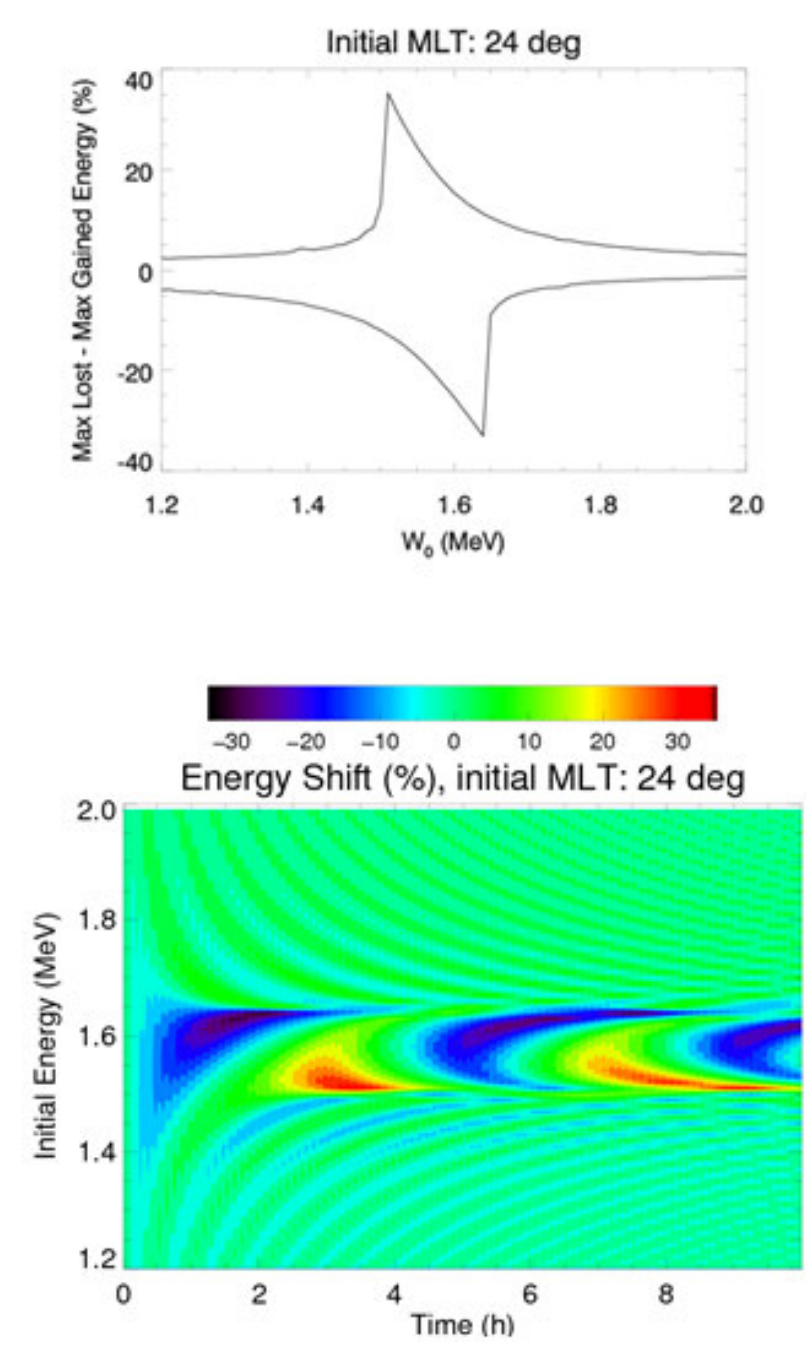

$\mathbf{m}=\mathbf{2}(\mathbf{f}=\mathbf{5} \mathbf{m H z})$

Initial MLT: 24 deg
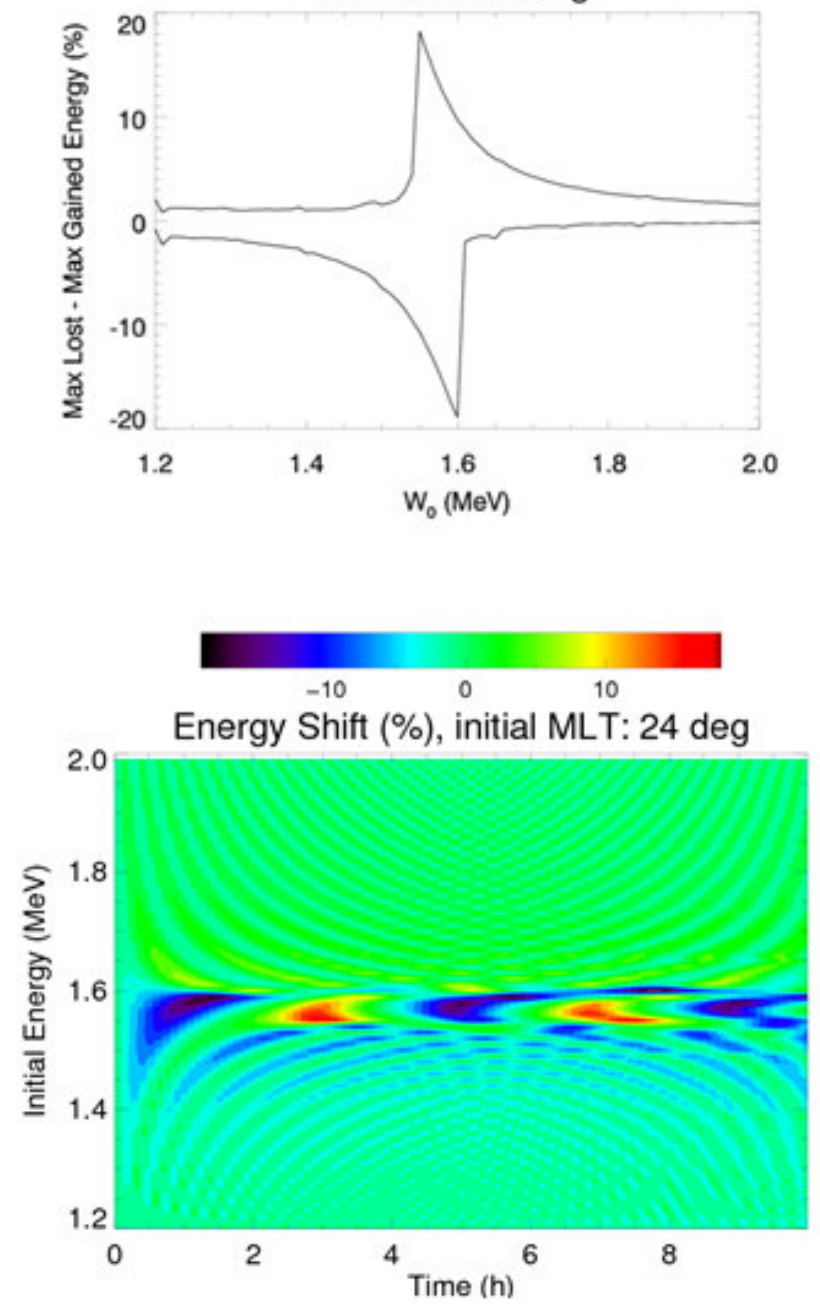

Figure 4-16a: Electron energy evolution by interacting with monochromatic wave confined in a sector. Initial placement: $\phi_{0}=24$ degrees. 


$$
\mathrm{m}=0(\mathbf{f}=2.5 \mathrm{mHz})
$$
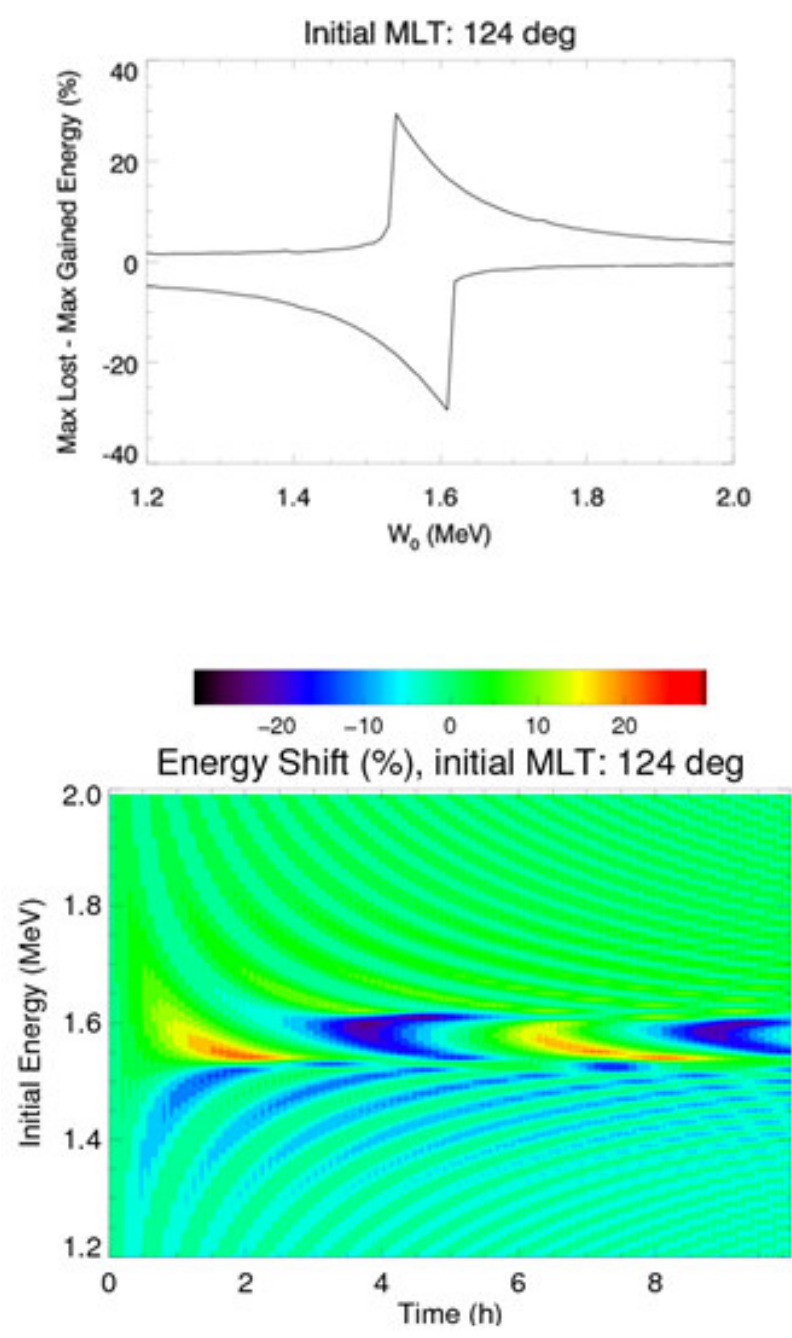

$\mathbf{m}=\mathbf{2}(\mathbf{f}=\mathbf{5} \mathbf{~ m H z})$

Initial MLT: $124 \mathrm{deg}$
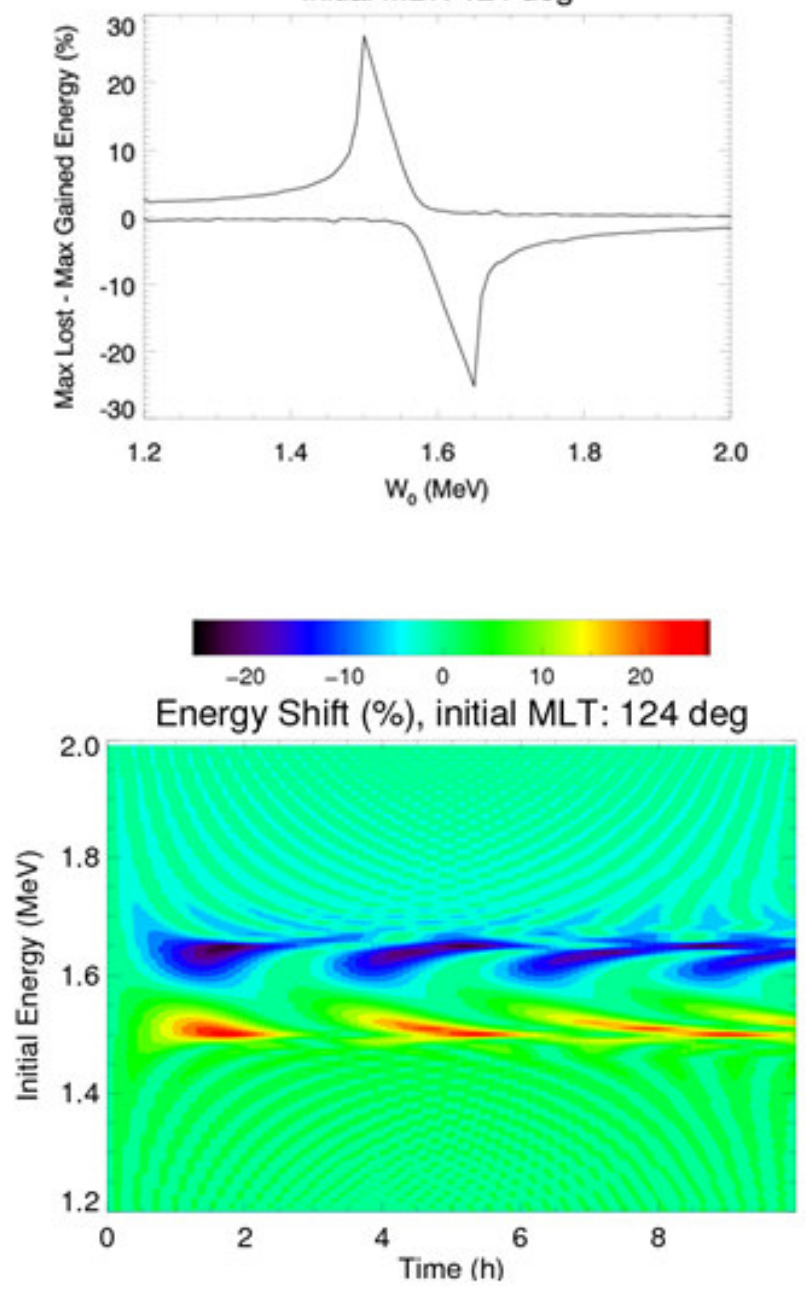

Figure 4-16b: Electron energy evolution by interacting with monochromatic wave confined in a sector. Initial placement: $\phi_{0}=124$ degrees. 


$$
\mathbf{m}=\mathbf{0}(\mathbf{f}=2.5 \mathrm{mHz})
$$
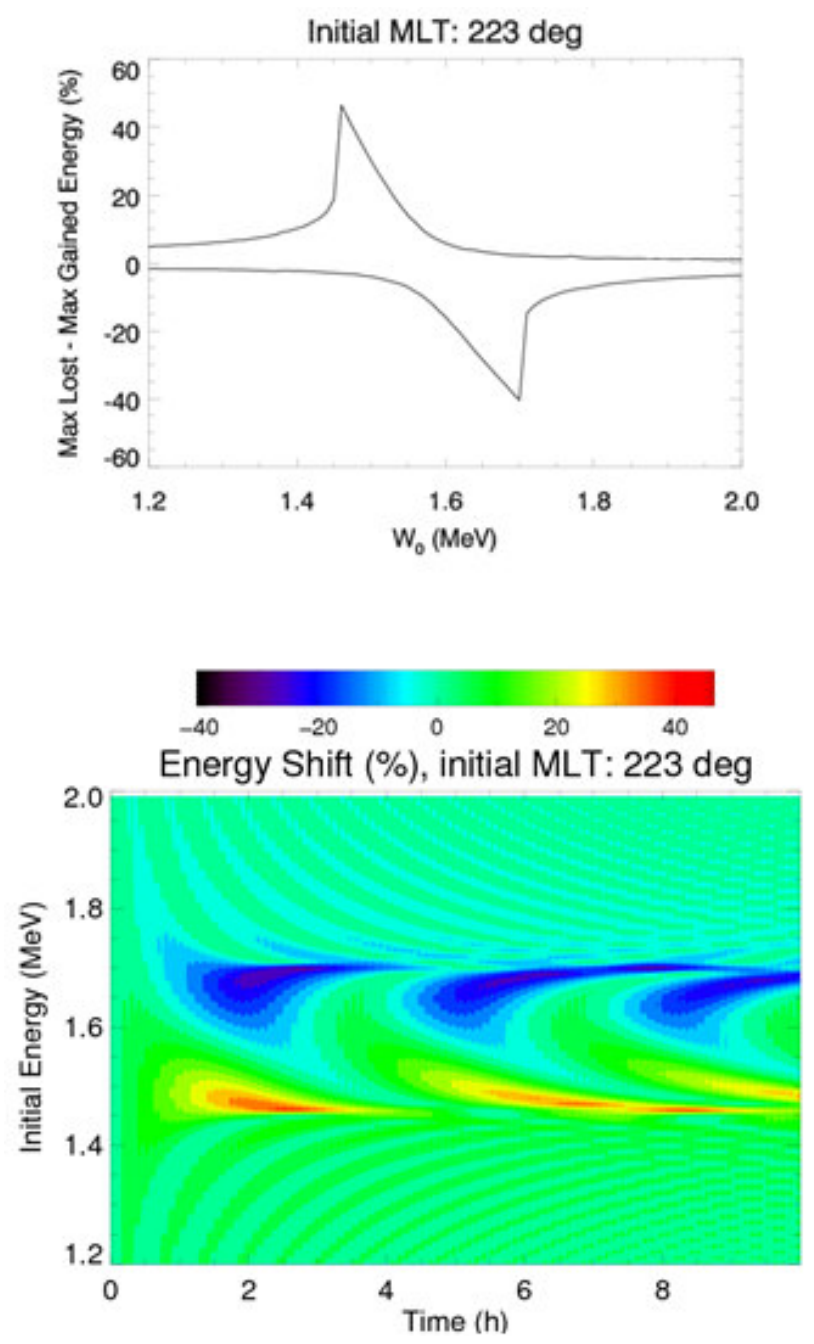

$\mathbf{m}=\mathbf{2}(\mathbf{f}=\mathbf{5} \mathbf{~ m H z})$
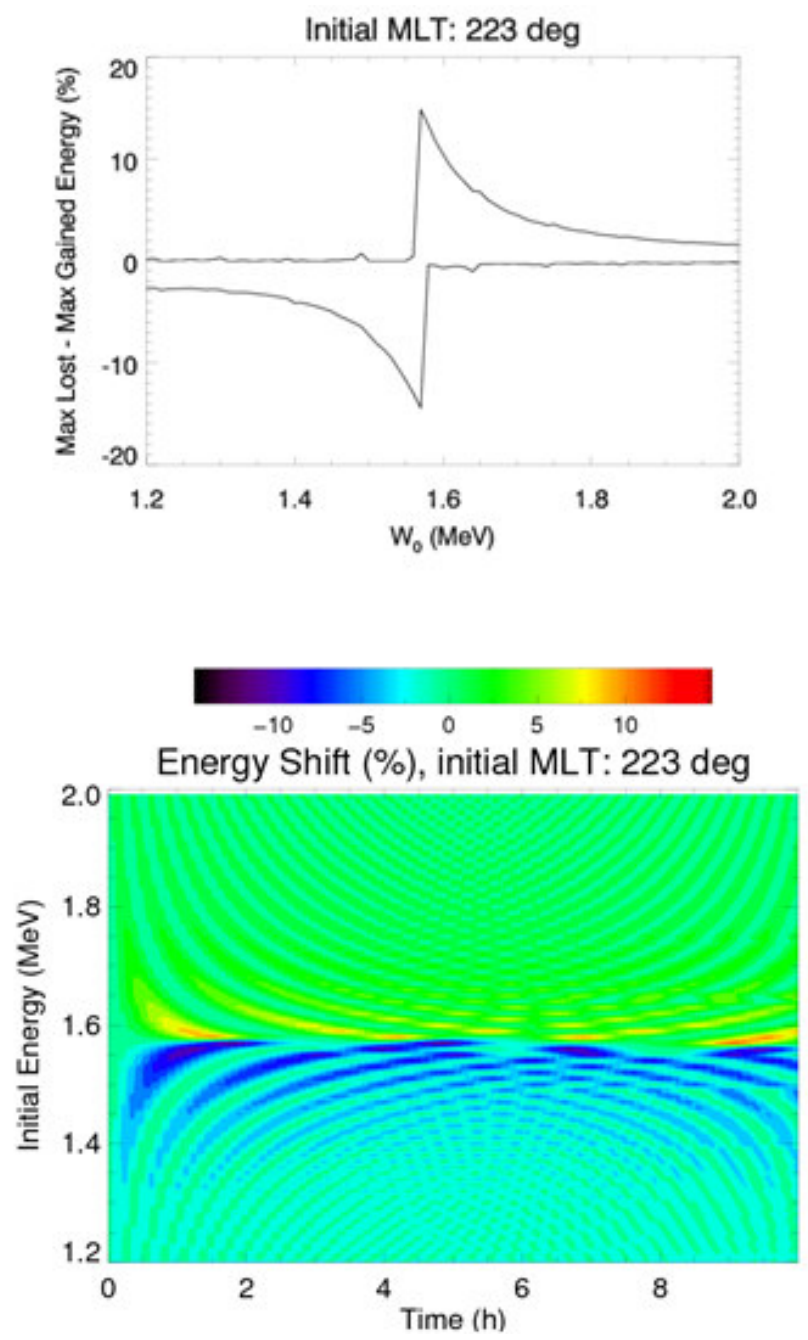

Figure 4-16c: Electron energy evolution by interacting with monochromatic wave confined in a sector. Initial placement: $\phi_{0}=223$ degrees. 


$$
\mathrm{m}=0(\mathbf{f}=2.5 \mathrm{mHz})
$$
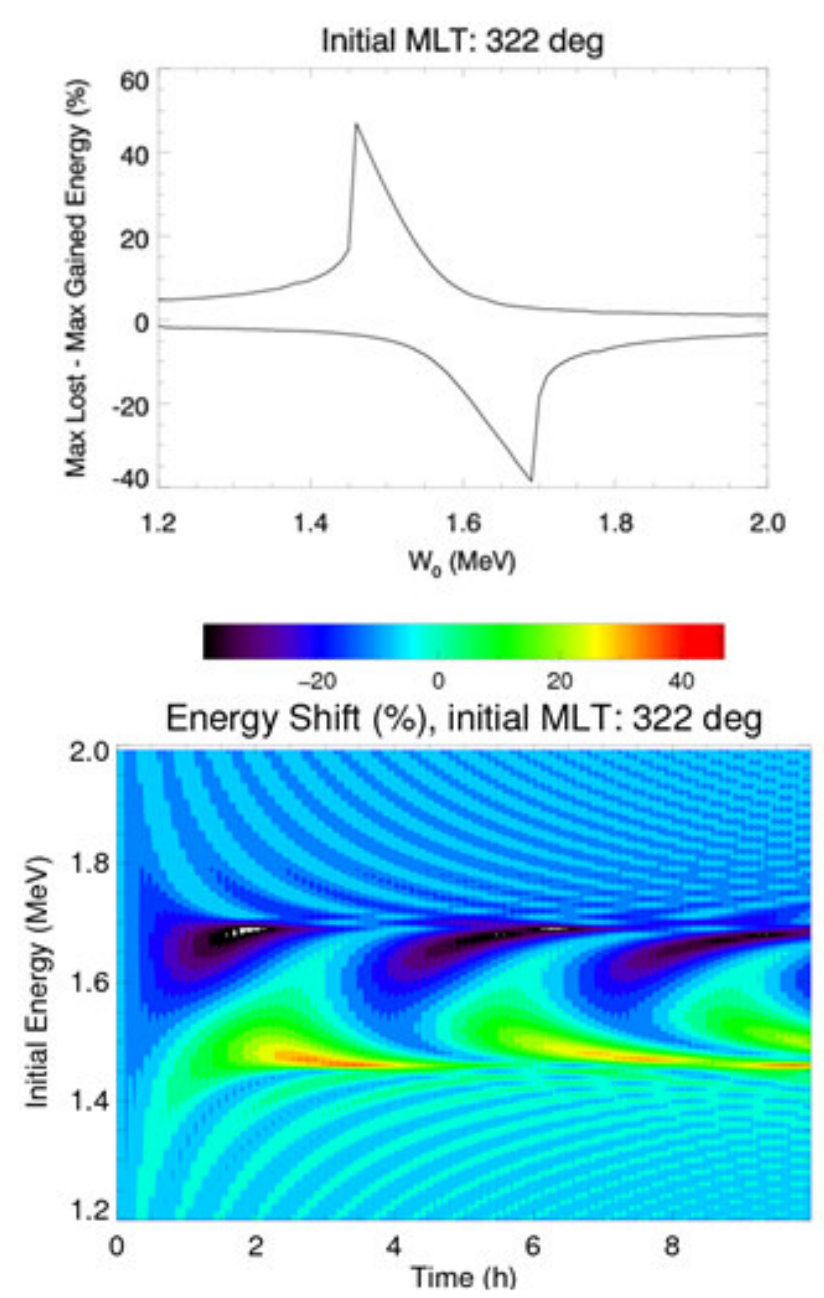

$\mathbf{m}=\mathbf{2}(\mathbf{f}=\mathbf{5} \mathbf{m H z})$
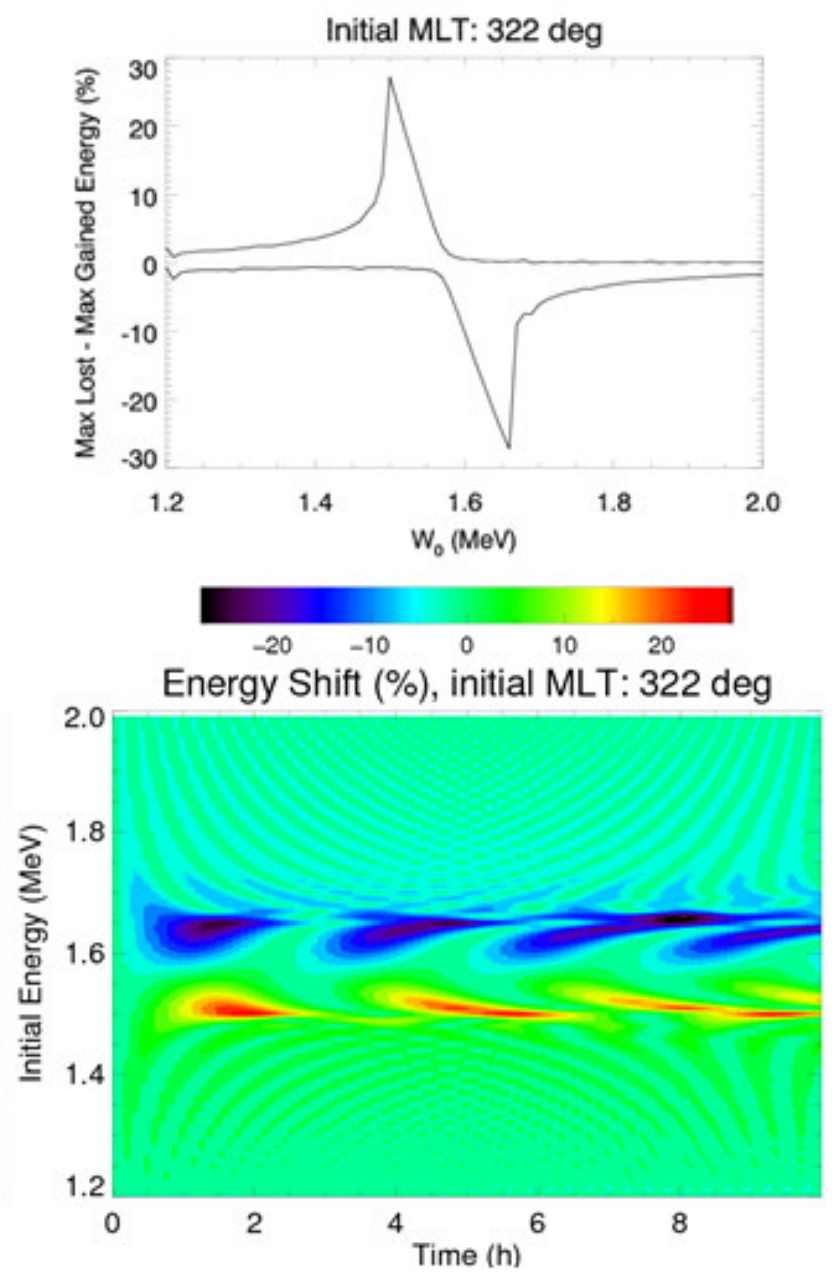

Figure 4-16d: Electron energy evolution by interacting with monochromatic wave confined in a sector. Initial placement: $\phi_{0}=322$ degrees. 
To find the work done on a particle in this configuration, in the same manner as for equation 4.2.3 or 4.2.5, we set the integration limit as the ULF wave power exposure time. Assuming a particle initially is launched at the boundary of the ULF region, the exposure time for one pass is:

$$
\tau=\frac{\Delta \phi_{\mathrm{sec}}}{\omega_{d}}
$$

Using standing waves the same integration as in 4.1 .5 becomes:

$$
\int_{0}^{\Delta \phi_{\mathrm{sec}} / \omega_{d}} E(\phi, t) d t=\frac{E_{0}}{2\left(\omega^{2}-m^{2} \omega_{d}^{2}\right)}\left[\begin{array}{l}
m \omega_{d}\left(\sin \left(\varphi_{d}-\varphi_{k}\right)+\sin \left(\varphi_{d}+\varphi_{k}\right)\right)- \\
-\left(\omega+m \omega_{d}\right) \sin \left(\frac{\left(\omega-m \omega_{d}\right)}{\omega_{d}} \Delta \phi_{\mathrm{sec}}+\varphi_{d}-\varphi_{k}\right)-\left(\omega-m \omega_{d}\right) \sin \left(\frac{\left(\omega+m \omega_{d}\right)}{\omega_{d}} \Delta \phi_{\mathrm{sec}}+\varphi_{d}+\varphi_{k}\right)
\end{array}\right]
$$

The resonant condition of $\omega-\mathrm{m} \omega_{\mathrm{d}}$ does not appear anymore, although there still is a resonant interaction between the oscillation and the particle. Additionally, since there is no wavenumber the spatial phase of the wave, $\phi_{\mathrm{d}}$, becomes meaningless and can be neglected. Let's assume that $\omega \sim \omega_{\mathrm{d}}$, leading to an identical oscillation phase at the next orbit. The integration becomes:

$$
\int_{0}^{\Delta \phi_{\mathrm{sec}} / \omega_{d}} E(\phi, t) d t=\frac{E_{0}}{2} \frac{\left[-\sin \left(\Delta \phi_{\mathrm{sec}}-\varphi_{k}\right)-\sin \left(\Delta \phi_{\mathrm{sec}}+\varphi_{k}\right)\right]}{\omega}
$$


However, the integration limit may need adjustments for the initial orbit. To give an example, the case where the launching position at 322 degrees the integration interval is

$$
t \in\left[0, \frac{\Delta \phi_{\mathrm{sec}}-\pi(322-270) / 180}{\omega_{d}}\right]
$$

since the sector begins at 270 degrees, and ends at 90 degrees. The sector width is $\Delta \phi_{\mathrm{sec}}=180 \mathrm{deg}$. The integration value becomes:

$$
\int_{0}^{\frac{\pi-\pi(322-270) / 180}{\omega_{d}}} E(\phi, t) d t=\frac{E_{0}}{2 \omega} 0.788
$$

thus a positive value is obtained, leading to an outward radial drift and loss of energy. It is evident this is exactly what occurs for all resonant electrons at the initial stage from figure 416d. This can be repeated again for the second pass, and so on, as long as the drift frequency does not stray too far apart from the wave frequency - it does change as transport occur, which is fully covered in the particle simulations only. As long as this criterion holds, the numerical value of (4.5.7) becomes the same or nearly the same at each orbital pass of the particle, thus the evaluated electron can be considered undergoing a resonant interaction with the wave.

This does not hold for very long in this case however, since less than an hour into the simulation when about half of the nonlinear oscillation period has been reached, the scattering process reverses. Although it does not work to use field integrations alone to predict resonant electron scattering for these longer time scales, this method will still be deployed in the next chapter for systems containing a large number of waves. 
When azimuthal wavenumbers, $\mathrm{m}$, are non-zero, the resonant condition is manifested in the denominator as $\omega_{\mathrm{k}}-\mathrm{m} \omega_{\mathrm{d}}$. So far we have assumed a wave spectrum which spans the entire azimuthal range and continuously scatters particles. For $\mathrm{m}=0$ when there is no azimuthal dependence in the path of the particles there cannot be any resonance in a globally distributed field. However, if it is assumed that the wave fields are confined in space, i.e. an azimuthal region, then a different type of resonance is made possible even for $m=0$, as described in chapter 4.4. A resonant particle passing through this region will only be exposed to a partial oscillation period during the first pass. Once it returns at a resonant drift period it will encounter the same phase of the oscillation and gain a net transport in the same direction as during the previous pass.

\subsection{Electron Interactions with a Wave Electromagnetic Field}

So far we have only discussed particle interactions with wave electric fields. Realistically, as

discussed in chapter 3 , ULF waves are composed of the components $E_{L}, E_{\phi}$ and $B_{z}$ that all contribute to particle scattering via Eq. (2.1.14) and (2.1.15). Thus we expand the discussion in this section to wave-particle interactions with a wave electromagnetic wave field.

For simplicity we consider a symmetric magnetic dipole $\left(\mathrm{B}_{\mathrm{c}}=0 \mathrm{nT}\right)$ so that only the $\mathrm{E}_{\phi}$ and $B_{z}$ components need to be regarded. The wave field is also set as global, i.e. no MLT dependence. As given previously, the wave function is a standing wave form:

$$
E_{\phi}=E_{0 \phi} \cos (\omega t) \cos \left(m \phi+\varphi_{d}\right)
$$


It can be evaluated with Faraday's law which would produce a self-consistent induced magnetic field of the form:

$$
B_{z}=-\frac{E_{0 \phi}}{\omega L} \sin (\omega t) \cos \left(m \phi+\varphi_{d}\right)
$$

and the gradients:

$$
\begin{gathered}
\frac{\partial B_{z}}{\partial \phi}=\frac{m E_{0 \phi}}{\omega L} \sin (\omega t) \sin \left(m \phi+\varphi_{d}\right) \\
\frac{\partial B_{z}}{\partial L}=\frac{E_{0 \phi}}{\omega L^{2}} \sin (\omega t) \cos \left(m \phi+\varphi_{d}\right)
\end{gathered}
$$

contribute to particle energization through the second terms in equation (2.1.14) and (2.1.15).

The drift velocities become:

$$
\begin{gathered}
v_{L}=\frac{E_{0} \cos (\omega t) \cos (m \phi+\varphi)}{\left[B_{0} / L^{3}-(E / \omega L) \sin (\omega t) \sin (m \phi+\varphi)\right]}+\frac{\gamma_{m} \mu \frac{m E_{0}}{\omega L^{2}} \sin (\omega t) \sin (m \phi+\varphi)}{\left[B_{0} / L^{3}-(E / \omega L) \sin (\omega t) \sin (m \phi+\varphi)\right]} \approx \\
\approx \frac{E_{0} L^{3}}{B_{0}} \cos (\omega t) \cos (m \phi+\varphi)+\gamma_{m} \mu \frac{m E_{0} L}{\omega B_{0}} \sin (\omega t) \sin (m \phi+\varphi) \\
v_{\phi} \approx \gamma_{m} \mu \frac{E_{0} L}{\omega B_{0}} \sin (\omega t) \cos (m \phi+\varphi)
\end{gathered}
$$

We conduct three separate test particle simulations with the possible combinations of wave field components: $\mathrm{E}_{\phi}, \mathrm{B}_{\mathrm{z}}$ and $\mathrm{E}_{\phi} \& \mathrm{~B}_{\mathrm{z}}$. A simulation time of $\tau_{\mathrm{sim}}=5 \mathrm{~h}$ ensures that all maximum energies can be reached, i.e. the nonlinear oscillation period (Eq. (2.2.9)) $\tau_{\mathrm{w}-\mathrm{d}}<\tau_{\mathrm{sim}}$ for all particles. We show the maximum electron energy gain for each initial condition in 
figure 4-17. To compare the effect on particle scattering by the wave components, we find $\max (\Delta \mathrm{W})$ as a function of the full EM wave, as well as of the separate $E_{\phi}$ and $B_{z}$ components.
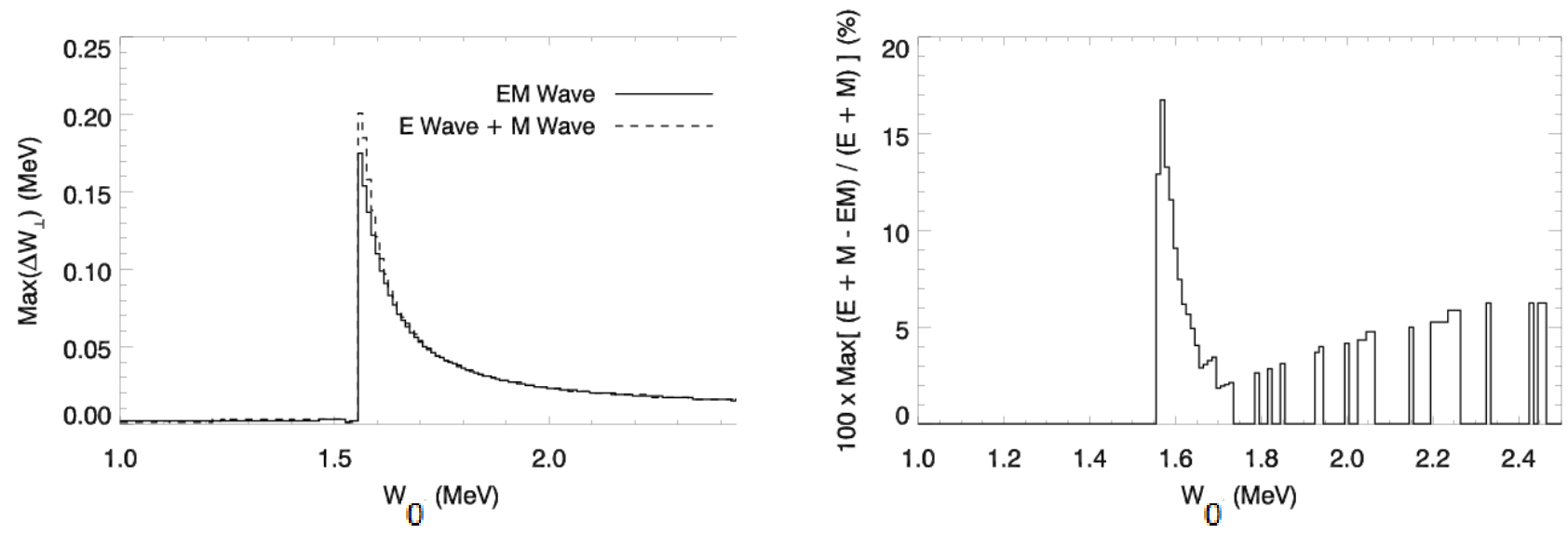

Figure 4-17: Left - Maximal energy gain due to wave-particle interaction for max $(\Delta W)_{E \phi}+$ $\max (\Delta W)_{B z}$ and $\max (\Delta W)_{E M}$. Right - Deviation in maximal energy reach between the contribution of an electromagnetic wave and the combined contributions from $E_{\phi}$ and $B_{z}$ components.

The maximum energy gain caused by the self-consistent EM wave field is about 10-15\% smaller than the maximum energy gain summed by the $\mathrm{E}_{\phi}$ and $\mathrm{B}_{\mathrm{z}}$ contributions. The difference arises from the fact that the $\partial \mathrm{B}_{\mathrm{Z}} / \partial \mathrm{L}$ gradient adjusts the electron azimuthal drift velocity, which in turn changes the relative phase between the particle and the wave. The radial displacements, derived by integrating equation (4.6.5), are then determined by such adjustments through the local wave power in the particle frame. 


\subsection{Conclusions}

Wave-particle interactions in the radiation belts can range from the simplest case with a monochromatic wave form with $\mathrm{E}_{\phi}$-polarization in a symmetric dipole, to including asymmetry, other components in the polarization, and coexisting multiple waves.

For the single wave case, we have demonstrated the departure from a single solution for a set of many initial phase space conditions for electrons, where the first adiabatic invariant becomes a parameter. Each island can be highly asymmetric about the resonance frequency, but the overall acceleration for the global population remains negligible.

It is first when 2 waves with overlapping resonance widths $(K>1$ ) coexist in the system when global acceleration becomes possible. The onset of acceleration occurs after about $\sim 1$ hour, and is followed by at least another 4 hours in this state, regardless of phases. The qualitative differences take form due to the phase of one of the two waves, with a higher scattering efficiency for $\phi_{1}=3 \pi / 2$ compared to $\phi_{1}=\pi / 2$.

The polarization of the waves is another significant factor. In our experiment with a dipole field asymmetric in MLT, we conclude that wave $\mathrm{E}_{\phi}$ components are 3-10 times more efficient compared to $\mathrm{E}_{\mathrm{L}}$ components depending on phase and amplitude for both the wave and the dayside magnetic compression. A fully self-consistent electromagnetic wave falls short of about $10-15 \%$ in scattering width compared to the added contributions from each component separately. 


\section{Stochastic Dynamics and Diffusion}

This chapter treats diffusive, long-term electron scattering as opposed to the short-term scattering discussed in chapter 4 . We employ a technique in calculating the radial variance from the wave fields, and verify the diffusion coefficients with test-particle simulations for broadband spectra. In addition to the local power spectral density as a driver of diffusion, as known before, we prove that dynamic phases, either in the particle frames or extrinsically produced, is a significant factor in diffusion. This effect is strongest for wave vectors perpendicular to the particle drift paths $(\mathrm{m}=0)$, but also non-negligible for low, non-zero wavenumbers. We show that magnetic local time sectors containing ULF activity adjusts the diffusion coefficient due to both dynamic phases as well as a function radial position making the radial gradient of the diffusion coefficient slightly less steep than known before.

\subsection{Introduction}

The previous chapter treated short-term acceleration of electrons at the order of hours up to half a day, but when going to even longer time scales over at least a day it becomes more practical to approximate particle scattering as diffusive. The main item that needs to be quantified for such systems is the diffusion coefficient in the diffusion equation (2.3.4). We discussed in the introduction in chapter 2-3 that this coefficient has been evaluated through both observations and models, where the main focus has been in the local power spectral density of the broadband wave fields, as well as the radial location of the scattering process. It 
was shown in the previous chapter that the phases can determine the overall scattering effect in a 2-wave-particle interaction. The choice of phase cannot have a significant influence on diffusion however since it is a statistically constructed quantity of multiple scattering instances, but it does raise the question in what manner the stochasticity in phases can contribute to the diffusion coefficient. Another parameter to be investigated in this chapter is the sector width, which has already been demonstrated for monochromatic wave-particle interaction in chapter 4-6. This also introduces a direct relation to the drift frequency, making the problem complex since this quantity is linked to resonances in the local power spectrum as well as the intermediate L-shell position.

\subsection{Stochasticity Effects on Wave-Particle Interactions}

\subsubsection{Effects of Intrinsic stochasticity}

We begin this chapter by demonstrating the effect of stochasticity in wave-particle interactions. The first experiment is by simulating electrons interacting with a global wave electric field of the standing form

$$
E_{\phi}(\phi, t)=\sum_{k=1}^{N_{k}} E_{0, k} \cos \left(\omega_{k} t+\varphi_{k}\right) \cos \left(m \phi+\phi_{0}\right)
$$

A way to cause diffusion in electron scattering is by introducing a broadband spectrum of modes. A good range here is $\mathrm{f}_{\mathrm{k}}=1-10 \mathrm{mHz}$, which covers almost the whole Pc5 band. 
A question that arises is how the frequency resolution $\Delta$ f should be defined. One suggested answer is to derive it from the solar wind spectrum [Ukhorskiy and Sitnov, 2008]. This is a power-law spectrum $\mathrm{f}^{\beta}$, with values typically at $-1<\beta<-2$. The lowest frequency portion is defined as the autocorrelation time for fluctuations in the interplanetary plasma and can be directly linked to $\Delta \mathrm{f}$ [ Burlaga, 1968], [Jokipii, 1968 ]. A typical value is $\Delta \mathrm{f}=0.1 \mathrm{mHz}$, which will be the default choice for all cases discussed in this chapter. Spectral amplitudes also matter in defining in $\Delta \mathrm{f}$; in some cases there can exist strong distinct modes with wide frequency separations, as opposed to a flat and uniform spectrum. One such example will be given in the case study in chapter 6 . Since we only assume uniform amplitudes in this chapter, $\Delta \mathrm{f}$ will always remain the same.

With $\Delta \mathrm{f}=0.1 \mathrm{mHz}$ the uniform amplitudes are set to $\mathrm{E}_{\mathrm{k}}=0.1 \mathrm{mV} / \mathrm{m}$ in order to maintain the stochasticity parameter $K>1$, thus beyond the onset for stochasticity. We assign a random phase for each mode that remains constant throughout each simulation. This type of stochasticity is intrinsic and implies there is a periodic decorrelation between particles and modes. The frequency step $\Delta \mathrm{f}$, as described in equation (2.3.1), gives a decorrelation time scale of $\tau_{\Delta \mathrm{f}}=1 /(0.1 \mathrm{mHz})=10,000$ seconds or 2.8 hours, which is comparable to the simulation time scale. Thus diffusion would only be evident for time scales at multiples of this Decorrelation time, or at the order of about 1 day for this and other similar systems. To achieve diffusive scattering, the simulation run could either be extended to fit a suitable time scale or alternatively be divided up into smaller range runs with random phases in each one. The latter is demonstrated here for 20 simulation runs of $\tau_{\text {sim }}=3.0$ hours each with unique sets of phases. If placed as a sequence the equivalence is a total simulation time of $\tau_{\text {tot }}=60$ hours $=2.5$ days . 
As was discussed in chapter 3-3 we need to calculate the variance of the radial position or of the energy for the particle population to find the diffusion coefficient, as per equation (2.3.5). It could be relative to either the initial or the mean value for the population. In this thesis the latter has been chosen in order to eliminate deviations due to asymmetric scattering in L-shell that occurs in this example (see fig 5-1, bottom). The graph in figure 5-1a shows the variance in radial position, $\left\langle\Delta \mathrm{L}^{2}\right\rangle$, for each separate simulation. The variance $\left\langle\Delta \mathrm{L}^{2}\right\rangle$ is not a linear function of time as one would expect in diffusive processes, but instead displays chaotic scattering produced by multiple simultaneous resonant interactions. However if the variance from all 20 simulations is averaged so that the effect of the initial phases cancels, a clear linear trend appears, as shown in figure 5-1b. The error margin is found by applying a standard deviation on $\left\langle\Delta \mathrm{L}^{2}\right\rangle_{\mathrm{e}}$ - for all simulations combined at each time step: $\sigma_{\text {sim }}(t)=\sqrt{\left\langle\left\langle\Delta L^{2}(t)\right\rangle_{e^{-}}\right\rangle_{\text {sim }}}$. The resulting diffusion coefficient derives from the linear fit of variance averaged over the simulations, $\overline{\left\langle\Delta L^{2}\right\rangle_{e^{-}}}$(fig. 5-1b, blue graph), and the error margins are given as the linear fits of $\overline{\left\langle\Delta L^{2}\right\rangle_{e^{-}}} \pm \sigma_{\text {sim }}^{2}$ (fig. 5-1b, red graphs). Thus the diffusion coefficient is:

$$
D_{L L}=\frac{\overline{\left\langle\Delta L^{2}\right\rangle_{e^{-}}} \pm \sigma_{\text {sim }}^{2}}{2 \tau}
$$



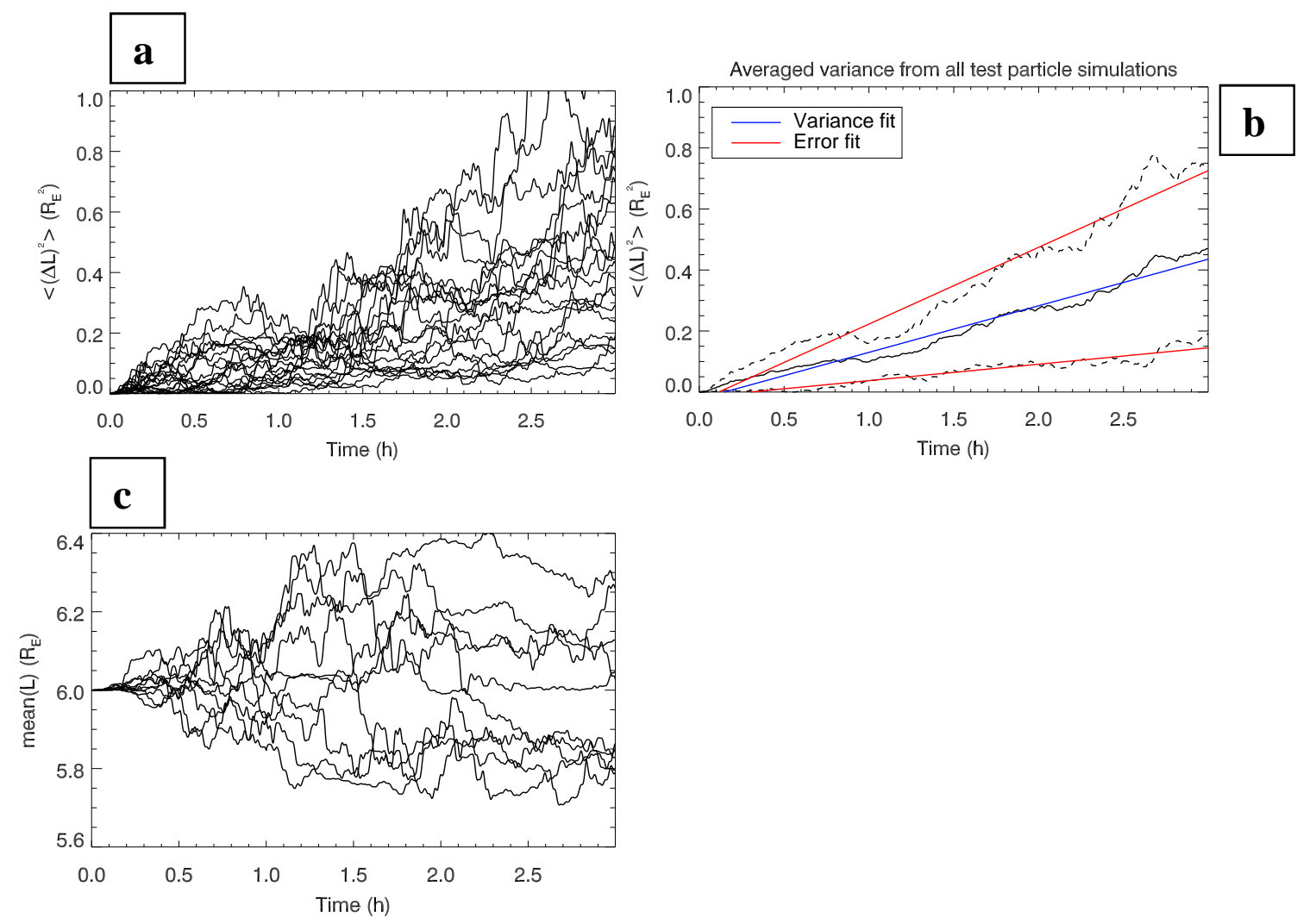

Figure 5-1: Time evolution of L-shell variance due to broadband spectra of random phases, $f_{k}=1-10 \mathrm{mHz}$. a) Individual simulations. $\left.\boldsymbol{b}\right)$ Averaged L-shell variance from 20 simulations as a function of time. c) Mean value of radial positions for 10 of the simulation sets.

\subsubsection{Effects of Extrinsic stochasticity}

Extrinsic stochasticity takes form as randomly reset phases. For example, the medium and dimensions of the magnetospheric space in which waves propagate can suddenly change, which would as a consequence adjust the phase velocity and frequency. Exterior pulsations in the solar wind that excite magnetospheric waves can also undergo random fluctuations that would directly transfer to within the magnetosphere. The period of random resets is arbitrary and can be shorter than the decorrelation period $\tau_{\Delta \mathrm{f}}$ found in section 5.2.2. 
In this section we will demonstrate electron scattering due to extrinsic stochasticity. For this purpose we consider a monochromatic mode interacting with electrons. The wave frequency is set to $\mathrm{f}_{1}=2 \mathrm{mHz}$ with $\mathrm{m}=0$ and placed in an MLT sector of width $\Delta \phi_{\mathrm{sec}}=\pi / 2-$ similar to the case in chapter 4-6. The initial conditions for the electrons are $L_{0}=4.6, \phi_{0}=0-2 \pi$ with $\Delta \phi=\pi / 50$, and $\mathrm{W}_{0}=0.1-4.0 \mathrm{MeV}$ with $\Delta \mathrm{W}=0.1 \mathrm{MeV}$, which gives a range $0.32 \leq \mu \leq 12.8 \mathrm{keV} / \mathrm{nT}$. Since there is only one wave in the system there cannot be any intrinsic stochasticity, so instead we impose randomization actively, i.e. extrinsic stochasticity, by resetting the wave phase at frequent intervals $1 / f_{\mathrm{r}}$. Four different phase reset frequencies are shown in fig. 5-2 in terms of their effect on electron scattering and diffusion. We find the radial position variance from 10 simulations with different phases for the wave. For the non-randomized phase (top left graph) the scattering is deterministic and reversible, therefore adiabatic transport occurs in each simulation. Even though 10 different simulations are averaged the outcome is statistically the same for each one since the global distribution of electrons averages out any phase effects during the wave-particle interaction. The resulting scattering is thus not defined as diffusion for these static phases, but scattering which is reversible after the decorrelation period. The scattering graphs in fig. 5-2 show harmonic peaks of $\mathrm{f}_{\mathrm{d}}=\mathrm{nf}_{1}$ and their amplitude decreases as $1 / \sqrt{n}$. 

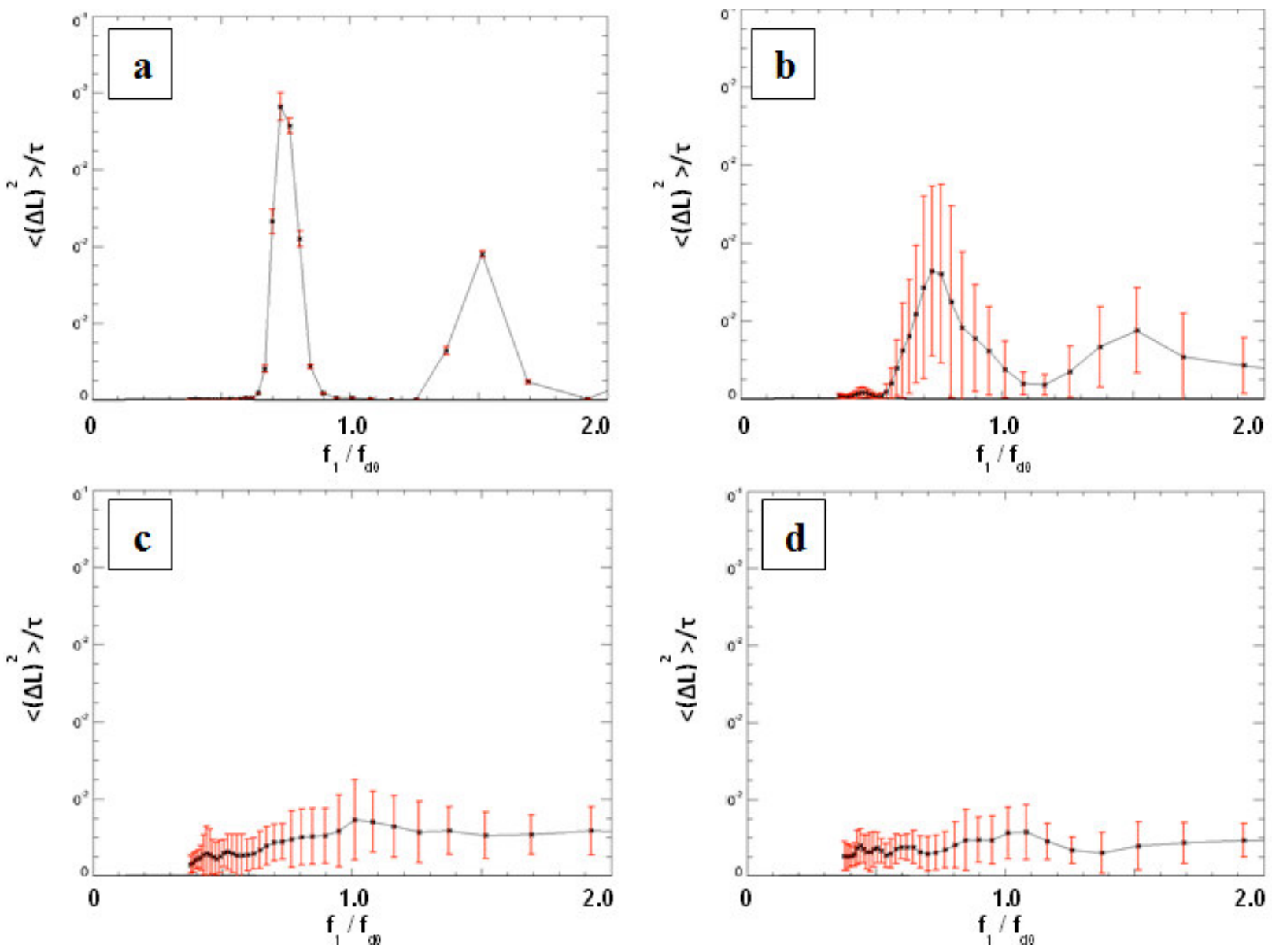

Figure 5-2: Electron scattering due to a monochromatic wave at $f_{1}=2 \mathrm{mHz}$ in 1 sectorscattering averaged over both electrons and simulations. a) No phase resets, b) Phase reset at the rate: $\left.\left.f_{r}=0.83 \mathrm{mHz}, \boldsymbol{c}\right) f_{r}=3.33 \mathrm{mHz}, \boldsymbol{d}\right) f_{r}=10 \mathrm{mHz}$.

At $f_{r}=0.83 \mathrm{mHz}$ (case $b$ ) the phases are randomized at a slower rate than the wave frequency, thus the resonance is still clearly in effect albeit weakened. The diffusion coefficient now contains contributions from both the deterministic and the stochastic scattering, and from the latter a large uncertainty appears. For even faster phase reset rates at $\mathrm{f}_{\mathrm{r}}=3.33 \mathrm{mHz}$ and $10 \mathrm{mHz}$ (fig. 5-2c-d) the resonance has been destroyed and the stochastic scattering has become more uniform as a function of the drift frequency. In the extreme case where every 
time step were random: $f_{r}=1 / \Delta \tau_{\text {sim }}$, the motion would be per definition Brownian and $D_{L L}$ would be completely independent of $f_{d}$.

We showed here examples for intrinsic and extrinsic stochasticity and their effects on the electron diffusion in radial position. Both types can exist in observed wave fields, and whichever causes the most rapid phase reset rate will dominate $\mathrm{D}_{\mathrm{LL}}$.

\subsection{Calculating Diffusion Coefficients via the Variance Method}

\subsubsection{The variance method}

An alternative and quicker way to evaluate $D_{L L}$ than test particle simulations is via integrating the drift velocities according to equation (2.3.7) to directly find the radial variance. These calculations require information on the radial velocities of electrons, which have contributions from the electric wave field components $E_{L}$ and $E_{\phi}$ as well as wave magnetic fields $B_{z}$ :

$$
v_{L}=\frac{L^{3}}{B_{z 0} R_{E}} E_{\phi}+\frac{\partial B / \partial \phi}{3 B_{z 0}^{2} R_{E}} L^{6} E_{L}+\frac{4}{3} \frac{\partial B_{z} / \partial \phi}{B_{z 0}^{2} R_{E}} L^{6} E_{\phi}-\frac{\gamma_{m} \mu L^{2}}{B_{z 0} R_{E}^{2}} \frac{\partial B_{z}}{\partial \phi}+\frac{8 \gamma_{m} \mu L^{5}}{3 B_{z 0}^{2} R_{E}^{2}} B_{c} \frac{\partial B_{z}}{\partial \phi}
$$

The second and third terms can be simplified, and the fifth term dropped, by neglecting the wave magnetic field component:

$$
v_{L}=\frac{L^{3}}{B_{z 0} R_{E}} E_{\phi}+\frac{B_{c} \cos \phi}{3 B_{z 0}^{2} R_{E}} L^{6} E_{L}+\frac{4}{3} \frac{B_{c} \cos \phi}{B_{z 0}^{2} R_{E}} L^{6} E_{\phi}-\frac{\gamma_{m} \mu L^{2}}{B_{z 0} R_{E}^{2}} \frac{\partial B_{z}}{\partial \phi}
$$


Note that in principle the radial drift is perpendicular to the magnetic contours, i.e. L*. If $\mathrm{B}_{\mathrm{c}} \neq 0 \mathrm{nT}$, the azimuthal drift velocity becomes a function of azimuthal position, which would give coupled differential equations that must be treated for exact solutions. To simplify the variance technique here, we always assume a symmetric magnetic dipole field in order to maintain a constant $\mathrm{v}_{\varphi}$ and also transform $\mathrm{L}^{*}$ to $\mathrm{L}$.

This function can now be divided into the contributions of wave electric fields into the first three terms, and wave magnetic fields into the last two terms. With an azimuthally symmetric dipole field the radial velocity is simplified to:

$$
v_{L}=\frac{L^{3}}{B_{z 0} R_{E}} E_{\phi}-\frac{\gamma_{m} \mu L^{2}}{B_{z 0} R_{E}^{2}} \frac{\partial B_{z}}{\partial \phi}
$$

We use the wave electric field (5.2.1) and, with the radial velocity function established, it is possible to calculate the variance of the radial scattering of an arbitrary population of electrons with equation (2.3.7). As mentioned in the introduction in chapter 2.3, Fälthammar (1965) eventually reached the result that the variance of the particle scattering is proportional to the power spectral density of the wave electric field. For example the case for wave $\mathrm{E}_{\varphi}$ field becomes:

$$
\left\langle(\Delta L)^{2}\right\rangle=\frac{L^{6}}{2 B_{z 0}^{2} R_{E}^{2}} \sum_{n=1}^{N} \int_{0}^{\infty}\left[E_{\phi, m}(t) E_{\phi, m}(t+\tau)\right] \cos m \omega_{d} \tau d \tau=\frac{L^{6}}{2 B_{z 0}^{2} R_{E}^{2}} \sum_{m} P_{m}^{E, s y m}\left(m \omega_{d}\right)
$$

The form of the power spectral density as the derivative of the PSD from the Fourier transform of the autocorrelation function is known as the Wiener-Khinchin theorem [Wiener \& Khinchin, 1934] where any phase information is integrated out in the Fourier spectrum, and thus has no effect on the final value. The result (5.3.4) then assumes a stationary and ergodic 
system where the phases of the modes are static over time scales at the order of $t>1 / \omega$. This is sufficient to calculate diffusion for a system where particles are exposed to the same wave field without phase randomizations or discontinuous amplitudes. Another factor is that the diffusion can only be found for waves with azimuthal mode numbers that are non-zero $(\mathrm{m} \neq 0)$. The third limitation is that $\mathrm{L}$ is assumed to be fixed at all times.

\subsubsection{Calculation of $D_{L L}$ for phase resets}

The analysis presented in the section 5.3.1 will be expanded here with the key difference being the use of randomizing phases. Additional extrinsic stochasticity can make an appearance through phase shifts in waves, as well as relative phases in geomagnetic positions of particles. Therefore this chapter will expand on the usage of a variance method to accommodate for short time intervals within which each phase reset period fits.

A modified azimuthal electric field of the form in (5.2.1) is applied to scatter the particles. The modification is an added dependence on the wave frequency as an inverse power law:

$$
E_{\phi}(t)=\sum_{k=1}^{N_{k}} \frac{2 \pi}{\xi(\beta) \omega_{k}^{\beta}} E_{0, k} \cos \left(\omega_{k} t+\varphi_{k, p}\right) \cos (m \phi)
$$

The term $\xi(\beta)$ is a normalization factor that ensures conservation of the total power in the spectrum for any spectral index $\beta$. It is derived from:

$$
P_{E}=\int_{f_{1}}^{f_{N_{k}}}\left|\tilde{E}_{\phi}\right| d f=\text { const }
$$


Performing this integral yields:

$$
\xi(\beta)=\frac{f_{N_{k}}^{(1-\beta)}-f_{1}^{(1-\beta)}}{\left(f_{N_{k}}-f_{1}\right)(1-\beta)}
$$

The phases $\phi_{\mathrm{k}, \mathrm{p}}$ comprise of a matrix of random values in the range of $[0,2 \pi]$ which are assigned to each mode, $\mathrm{k}$, and particle, $\mathrm{p}$. A number of particles for each L-shell and energy are then summed up in equation (5.3.4) where $\mathrm{N}=\mathrm{N}_{\mathrm{p}}$. In this testbed each particle represents a different initial condition or even a different scenario for each index $\mathrm{p}$ in order to maximize the accuracy of the diffusion coefficient. No additional random phase is necessary to add in the second cosine term since the single degree of parametric freedom is the relative, combined phases between the waves and the particles $\left(\phi_{\text {rel }}=\phi_{\text {wave }}-\phi_{\text {particle }}\right)$

This wave function can be inserted in the variance equation (5.3.4):

$$
\begin{aligned}
& \left\langle\left(L-L_{0}\right)^{2}\right\rangle=\frac{2 L^{6}}{B_{z 0}^{2} R_{E}^{2}} \int_{\tau=0}^{t} \int_{\zeta=0}^{\tau}\left\langle E_{\phi}(\tau) E_{\phi}(\varsigma)\right\rangle d \varsigma d \tau= \\
& =\frac{2 L^{6}}{B_{z 0}^{2} R_{E}^{2}} \int_{\tau=0}^{t} \int_{\zeta=0}^{\tau}\left\langle\sum_{k=1}^{N_{k}} \frac{E_{0}}{\xi(\beta) \omega_{k}^{\beta}} \cos \left(\omega_{k} \tau_{r}+\varphi_{k, p}\right) \cos \left(m \omega_{d} \tau_{r}\right) \frac{E_{0}}{\xi(\beta) \omega_{k}^{\beta}} \cos \left(\omega_{k} \varsigma+\varphi_{k, p}\right) \cos \left(m \omega_{d} \varsigma\right)\right\rangle d \varsigma d \tau= \\
& =\frac{2 L^{6} E_{0}^{2}}{\xi^{2}(\beta) B_{z 0}^{2} R_{E}^{2}} \frac{1}{N_{p}} \sum_{p=1}^{N_{p}} \sum_{k=1}^{N_{k}} \frac{\psi\left(\omega_{k}, \omega_{d}, \tau_{r}, \varphi_{k, p}\right)}{16 \omega_{k}^{2 \beta}\left(\omega_{k}^{2}-m^{2} \omega_{d}^{2}\right)^{2}}
\end{aligned}
$$

where

$$
\begin{aligned}
\psi\left(\omega_{k}, \omega_{d}, t, \varphi_{k, p}\right)= & \psi_{0}\left(\omega_{k}, \omega_{d}, t, \varphi_{k, p}\right)+\psi_{1}\left(\omega_{k}, \omega_{d}, t, \varphi_{k, p}\right)+\psi_{2}\left(\omega_{k}, \omega_{d}, t, \varphi_{k, p}\right)+ \\
& \psi_{3}\left(\omega_{k}, \omega_{d}, t, \varphi_{k, p}\right)+\psi_{4}\left(\omega_{k}, \omega_{d}, t, \varphi_{k, p}\right)+\psi_{5}\left(\omega_{k}, \omega_{d}, t, \varphi_{k, p}\right)
\end{aligned}
$$




$$
\begin{aligned}
& \psi_{0}\left(\omega_{k}, \omega_{d}, \varphi_{k, p}\right)=6 \omega_{k}^{2}+2 m^{2} \omega_{d}^{2}-4 \omega_{k}^{2} \cos \left(2 \varphi_{k, p}\right) \\
& \psi_{1}\left(\omega_{k}, \omega_{d}, t, \varphi_{k, p}\right)=2\left(\omega_{k}^{2}-m^{2} \omega_{d}^{2}\right)\left[\cos \left(2 m \omega_{d} t\right)-\cos \left(2 \omega_{k} \tau_{r}+2 \varphi_{k, p}\right)\right] \\
& \psi_{2}\left(\omega_{k}, \omega_{d}, t, \varphi_{k, p}\right)=-\left(\omega_{k}+m \omega_{d}\right)^{2} \cos \left[2\left(\omega_{k}-m \omega_{d}\right) t+2 \varphi_{k, p}\right] \\
& \psi_{3}\left(\omega_{k}, \omega_{d}, t, \varphi_{k, p}\right)=-\left(\omega_{k}-m \omega_{d}\right)^{2} \cos \left[2\left(\omega_{k}+m \omega_{d}\right) t+2 \varphi_{k, p}\right] \\
& \psi_{4}\left(\omega_{k}, \omega_{d}, t, \varphi_{k, p}\right)=4\left(\omega_{k}^{2}+m \omega_{d} \omega_{k}\right)\left\{\cos \left[\left(\omega_{k}-m \omega_{d}\right) t+2 \varphi_{k, p}\right]-\cos \left[\left(\omega_{k}-m \omega_{d}\right) t\right]\right\} \\
& \psi_{5}\left(\omega_{k}, \omega_{d}, t, \varphi_{k, p}\right)=4\left(\omega_{k}^{2}-m \omega_{d} \omega_{k}\right)\left\{\cos \left[\left(\omega_{k}+m \omega_{d}\right) t+2 \varphi_{k, p}\right]-\cos \left[\left(\omega_{k}+m \omega_{d}\right) t\right]\right\}
\end{aligned}
$$

The phases assume new random values at defined fixed time intervals $\tau_{\mathrm{r}}$, which can be multiplied to a total integrated time so that $t=N_{r} \tau_{r}$. We can replace the integration limit with $\tau_{\mathrm{r}}$ and multiply the function by $\mathrm{N}_{\mathrm{r}}$ to gain an approximation for $\left\langle(\Delta L)^{2}\right\rangle$. The assumption is that the scattering rate will remain constant, as the L-shell positions are fixed.

The variance now looks like:

$$
\left\langle(\Delta L)^{2}\right\rangle=\frac{2 L^{6} E_{0}^{2}}{\xi^{2}(\beta) B_{z 0}^{2} R_{E}^{2}} \frac{N_{r}}{N_{p}} \sum_{p=1}^{N_{p}} \sum_{k=1}^{N_{k}} \frac{\psi\left(\omega_{k}, \omega_{d}, \tau_{r}, \varphi_{k, p}\right)}{16 \omega_{k}^{2 \beta}\left(\omega_{k}^{2}-m^{2} \omega_{d}^{2}\right)^{2}}
$$

And thus the diffusion coefficient for the scenario of fixed phase reset rates can directly be found with equation (2.3.5) using this expression. $\mathrm{N}_{\mathrm{r}}$ drops out due to division by the total time, resulting in: 


$$
D_{L L}=\frac{L^{6} E_{0}^{2}}{16 \xi^{2}(\beta) B_{z 0}^{2} R_{E}^{2}} \frac{1}{N_{p}} \frac{1}{\tau_{r}} \sum_{p=1}^{N_{p}} \sum_{k=1}^{K} \frac{\psi\left(\omega_{k}, \omega_{d}, \tau_{r}, \varphi_{k, p}\right)}{\omega_{k}^{2 \beta}\left(\omega_{k}^{2}-m^{2} \omega_{d}^{2}\right)^{2}}
$$

If we assume a large number of particles so that $N_{p} \rightarrow \infty$, and a uniform distribution of random phases, the diffusion coefficient can be simplified into a final form:

$$
D_{L L}=\frac{L^{6} E_{0}^{2}}{16 \xi^{2}(\beta) \tau_{r} B_{z 0}^{2} R_{E}^{2}} \sum_{k=1}^{N_{k}} \frac{\psi\left(\omega_{k}, \omega_{d}, \tau_{r}\right)}{\omega_{k}^{2 \beta}\left(\omega_{k}^{2}-m^{2} \omega_{d}^{2}\right)^{2}}
$$

where

$$
\begin{aligned}
& \psi\left(\omega_{k}, \omega_{d}, \tau_{r}\right)=6 \omega_{k}^{2}+2 m^{2} \omega_{d}^{2}+2\left(\omega_{k}^{2}-m^{2} \omega_{d}^{2}\right)\left[\cos \left(2 m \omega_{d} \tau_{r}\right)-\cos \left(2 \omega_{k} \tau_{r}\right)\right]- \\
& -\left(\omega_{k}+m \omega_{d}\right)^{2} \cos \left(2\left(\omega_{k}-m \omega_{d}\right) \tau_{r}\right)-\left(\omega_{k}-m \omega_{d}\right)^{2} \cos \left(2\left(\omega_{k}+m \omega_{d}\right) \tau_{r}\right)
\end{aligned}
$$

A simpler expression is obtained for $\mathrm{m}=0$ :

$$
D_{L L}=\frac{L^{6} E_{0}^{2}}{2 \xi^{2}(\beta) \tau_{r} B_{z 0}^{2} R_{E}^{2}} \sum_{k=1}^{N_{k}} \frac{\left[1-\cos \left(\omega_{k} \tau_{r}\right)\right]}{\omega_{k}^{2 \beta} \omega_{k}^{2}}
$$

The phases do not need to be completely randomized for each interval. There can be scenarios similar to the case in chapter 4.6 where the wave field spectral power is concentrated in an MLT sector. Particles passing through MLT sectors with wave fields experience different phases depending on the initial azimuthal position. After each drift orbit period the particles encounter a new phase for every mode if $f_{d} \neq f_{k}$, and will scatter at a different magnitude. These phases are averaged out the same way as for randomized ones for $N_{p} \rightarrow \infty$. Equation (5.3.12) can be used to find diffusive scattering for this case as well. Instead of a constant 
reset time the time segments are dependent on the drift frequency as well as the azimuthal width of the sector:

$$
\tau_{r}=\frac{\Delta \phi_{\text {sector }}}{\omega_{d}}
$$

This type of scattering effect is non-resonant where any mode can affect any particle. The phase shifts are deterministic for individual particles, thus stochasticity is not involved. Therefore an element of stochasticity is still required to onset diffusion. The variance method ignores this by only allowing calculations for the initial drift orbit, but in the test particle simulations phase resets must be introduced at intervals at least as short as the recurrence rate of phases for resonant particles.

In summary the approach in using equation (5.3.11) is a general way to quickly approximate the diffusion coefficient for any wavenumber and any number of waves. There are however limitations as has already been hinted.

One must assign drift frequencies for the electrons and due to the resonant condition the result can be sensitive to that choice for $|m| \neq 0$. Let us assume $|\mathrm{m}|=1$ to begin with, then setting $\omega_{d}=\omega_{k}+\delta \omega$ gives $D_{L L} \rightarrow \infty$ as $\delta \omega \rightarrow 0$, and $D_{L L} \rightarrow-\infty$ as $\delta \omega \rightarrow \Delta \omega_{k}$. The middle point at $\delta \omega=\Delta \omega_{k} / 2$ gives a median value between the two extremes and is the exact solution for the averaged electron ensembles. For $|m| \neq 0,1$ the offset values must be adjusted by $\delta \omega \neq \Delta \omega_{k} / 2 m$ in order to ensure a central placement of $f_{d}$ between adjacent wave frequencies. If the offset is not properly assigned there is an adjustment to the magnitudes of $\mathrm{D}_{\mathrm{LL}}$ that will not mirror a real physical system; however the function versus frequency will 
remain the same and correct. The limitation due to this is that one can only calculate $\mathrm{D}_{\mathrm{LL}}$ for electrons at different initial drift frequencies with a resolution of $\Delta \omega_{k}$. It is impossible to evaluate the magnitude of $\mathrm{D}_{\mathrm{LL}}$ for the special case $\mathrm{k}=1$ since $\Delta \omega_{k}$ does not exist.

The sensitivity to initial drift frequencies also hinges together with the assumption that $\mathrm{L}$ is constant, whereas the particle simulations solve the differential equations for a variable $\mathrm{L}=\mathrm{L}(\mathrm{t})$. This yields issues if the wave amplitudes are too large since the electrons would experience shifts in drift frequencies so that the approximations do not hold any longer. Furthermore, since all phases for particles and modes are averaged together for initial drift orbit calculations, the model works only as if the wave fields are assigned new values for subsequent drift orbits. The loss of phase information in this case does diminish the importance of resonant, narrowband peaks as will become clear in chapter 6 . On the positive side, this method is several orders of magnitude quicker in computing $\mathrm{D}_{\mathrm{LL}}$ compared to the test particle simulations, allowing for a much wider range of parameters to be mapped in shorter computation time.

\section{4 $\mathrm{D}_{\mathrm{LL}}$ for global ULF oscillations}

The simplest example in which to calculate radial variances is the case of global, oscillating modes, in a symmetric magnetic dipole field without any wavenumbers, i.e. $\mathrm{m}=0$. Essentially this is a one-dimensional problem since there is no dependence on any spatial information and the particles experience the same oscillation regardless of their position. In magnetospheric physics there is no true application for this type of oscillation - instead this 
can be viewed as a test case for phase resets in general. Alternatively it can be viewed as the Doppler-shifted oscillations that particles experience in the local frame, noted as $\omega=\omega_{\mathrm{k}}-\mathrm{m} \omega_{\mathrm{d}}$, for cases where $|m| \neq 0$.

We calculate the diffusion coefficient by using the variance technique as outlined in chapter 5.2. A calculated example is shown in fig 5-3a for a monochromatic oscillation where the frequency is $\mathrm{f}_{0}=2 \mathrm{mHz}$, and the amplitude $\mathrm{E}_{0}=0.1 \mathrm{mV} / \mathrm{m}$. The phase undergoes random resets at frequencies $f_{r}=1 \mathrm{~Hz}$ to $1 \mathrm{mHz}$. The choice of modeling time scales and number of particles will be similar for the other variance calculations in this chapter and the next. We verify the result from the analytical approach by comparing with guiding center simulations. The average from a set of 10 simulations, each containing 200 electrons, with randomly generated wave phases also gives error margins the same way as in section 5.2. 

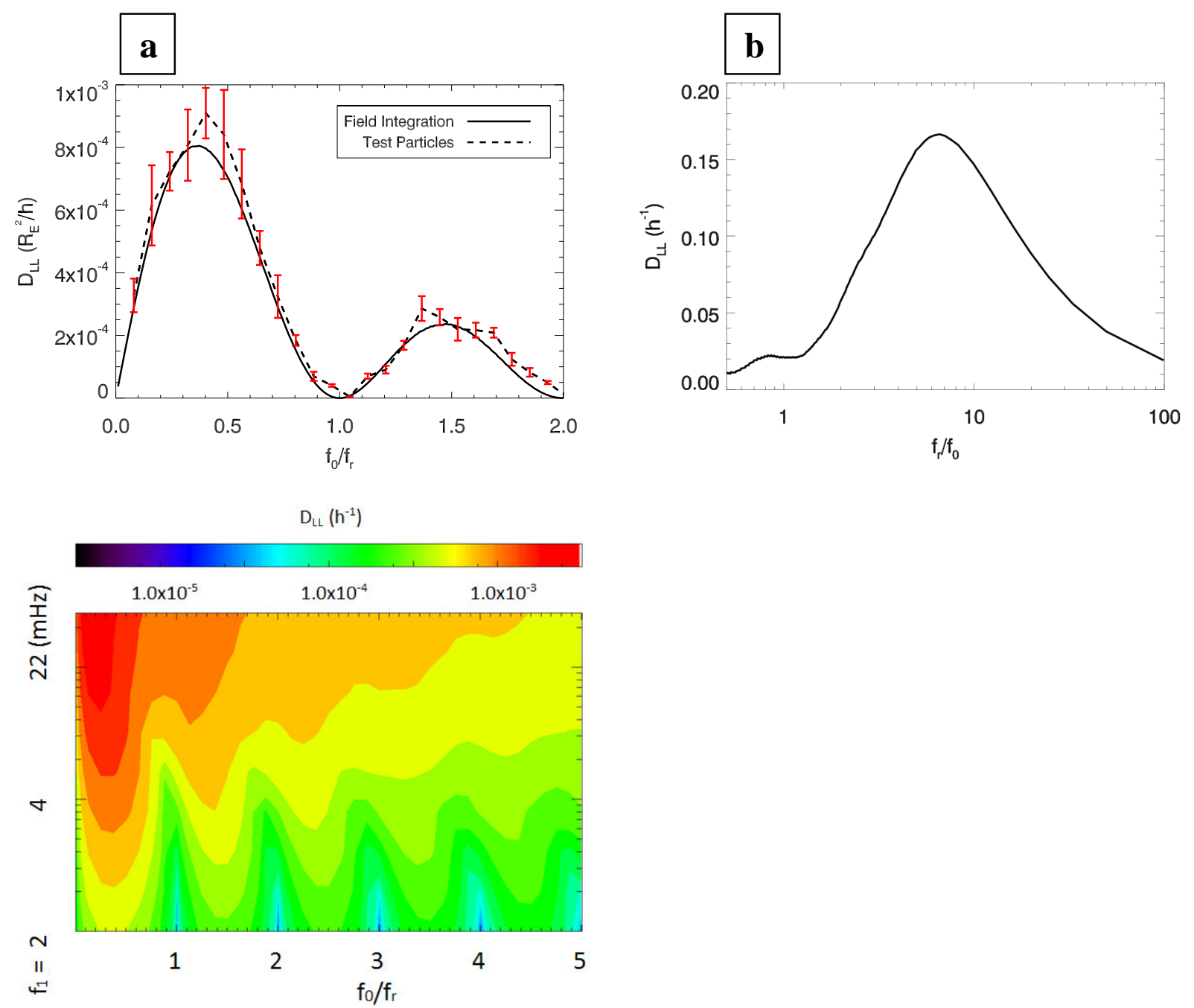

Figure 5-3: Analytical $D_{L L}$ caused by mode randomization at frequencies $1 \mathrm{mHz}<\mathrm{f}_{\mathrm{r}}<1 \mathrm{~Hz}$ for: a) single mode, $f_{1}=2 \mathrm{mHz}$, b) Multiple modes, $f_{k}=2-12 \mathrm{mHz}$, Bottom: As a function of broadband range.

For $1 / \tau_{\mathrm{r}}=\mathrm{f}_{\mathrm{r}}=1 \mathrm{~Hz}$, in other words for a ideal Brownian motion where a random jump occurs at every time step $\Delta \tau=1$ second, the diffusion coefficient is $\mathrm{D}_{\mathrm{LL}} \sim 10^{-6} \mathrm{~h}^{-1}$. The first maximum in $\mathrm{D}_{\mathrm{LL}}$ occurs at the ratio of $\mathrm{f}_{\mathrm{r}} / \mathrm{f}_{1}=2.63$ and is 2 orders of magnitude larger compared to the Brownian motion. Periodic minima exist at $\mathrm{f}_{\text {wave }}=\mathrm{nf}_{\mathrm{r}}, \mathrm{n}=1,2,3, \ldots$ where sinusoidal terms at (5.3.12) go to zero. The shape of the curve is asymmetric and approaches a sinusoidal function with decreasing $f_{r}$. For large of values of $f_{r}$ the function approaches a linear form 
where a best fit for $0.1<\mathrm{f}_{1} / \mathrm{f}_{\mathrm{r}}<2.5$ gives $\mathrm{D}_{\mathrm{LL}} \alpha\left(\mathrm{f}_{\mathrm{r}} / \mathrm{f}_{1}\right)^{1.04 \pm 0.14}$. This agrees with the general function form in (5.3.12): $\left(1-\cos \left(\omega \tau_{r}\right)\right) / \tau_{r}$, which is closely related to the sinc function.

We then assume a new wave electric field, defined by a broadband spectrum - in this case built up by uniformly spaced modes for $\mathrm{f}_{\mathrm{k}}=1-4 \mathrm{mHz}, \Delta \mathrm{f}=0.1 \mathrm{mHz}$, and still $\mathrm{m}=0$ considered. The amplitude of each mode is set to the same value as for the single wave case: $\mathrm{E}_{\phi \mathrm{k}}=0.1 \mathrm{mV} / \mathrm{m}$. We set each mode in the spectrum to experience reset rates ranging $1 \mathrm{mHz}<\mathrm{f}_{\mathrm{r}}<1 \mathrm{~Hz}$. The profile of $\mathrm{D}_{\mathrm{LL}}$ takes on a different shape as seen in figure 5-3b. The diffusion coefficient has local maxima again, although the frequencies of those have been shifted to higher values. Since each mode is equal in amplitude $(\beta=0)$ they all contribute equally to the diffusion coefficient for their respective frequencies. The peak $\mathrm{D}_{\mathrm{LL}}$ is found at:

$$
\left.f_{r}\right|_{\max \left(D_{L L}\right)}=2.63 \frac{\left(f_{1}+f_{N_{k}}\right)}{2}
$$

which in this case equals to: $f_{r}=6.58 \mathrm{mHz}$ - almost matching the peak in the analytical calculations found at $f_{r}=6.67 \mathrm{mHz}$. Just as for the monochromatic oscillation, the best fit for $\mathrm{D}_{\mathrm{LL}}$ within the frequency range $0.1<\mathrm{f}_{0} / \mathrm{f}_{\mathrm{r}}<5.0$ gives $\mathrm{D}_{\mathrm{LL}} \alpha\left(\mathrm{f}_{\mathrm{r}} / \mathrm{f}_{0}\right)^{1.05 \pm 0.01}$. 

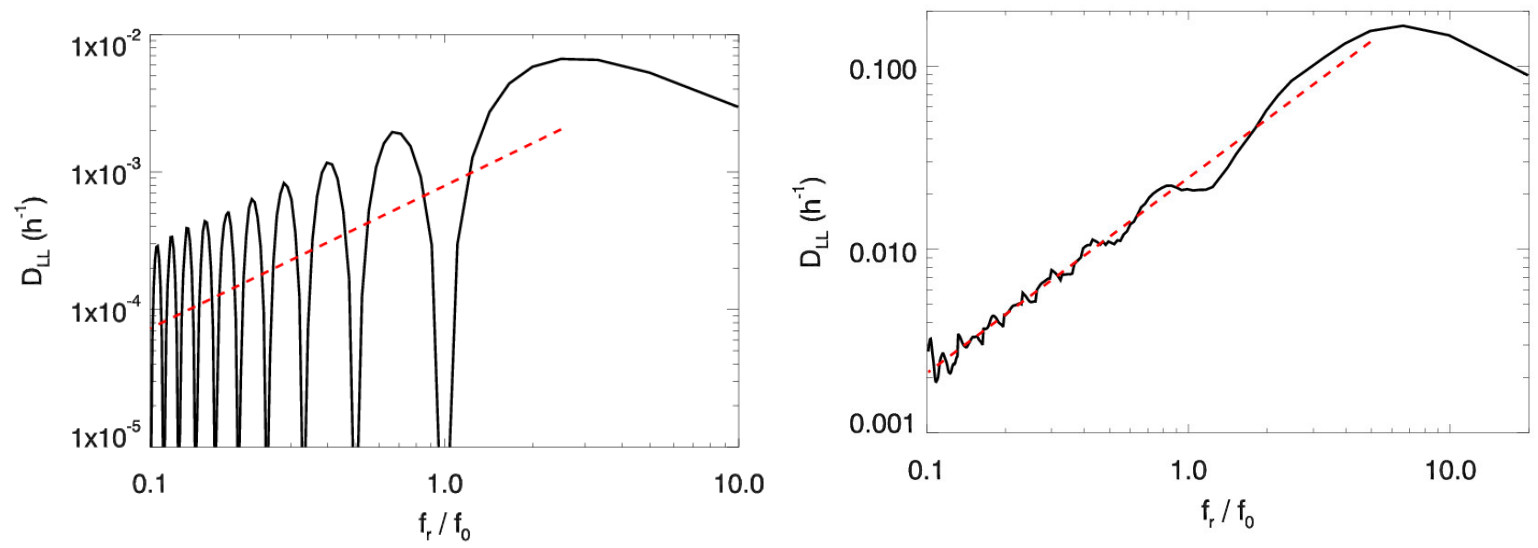

Figure 5-4: Left (case a): Diffusion coefficient for a wave electric field single mode at $f_{0}=2 \mathrm{mHz}$ with a periodically randomizing phase with reset frequencies, $1 \mathrm{mHz}<\mathrm{f}_{\mathrm{r}}<1 \mathrm{~Hz}$

Right (case b): same for multiple modes, $1<f_{k}<4 \mathrm{mHz}$.

\section{5 $\mathrm{D}_{\mathrm{LL}}$ for waves in local-time sectors}

\subsubsection{Demonstration with test particle simulations and variance calculations}

This section expands on the concept of regionally confined wave activity in azimuthal sectors as a driver of diffusive scattering. The azimuthal sector division that was described in chapter 4.4 is used for this discussion as well. We demonstrate the reliability of the variance method by comparing with test particle simulations in two different cases:

a) $\mathrm{f}_{\mathrm{k}}=2-10 \mathrm{mHz}, \Delta \mathrm{f}=0.1 \mathrm{mHz}, \mathrm{E}_{\phi 0, \mathrm{k}}=0.13 \mathrm{mV} / \mathrm{m}$ and $\mathrm{m}=0$.

b) $\mathrm{f}_{\mathrm{k}}=1-4 \mathrm{mHz}, \Delta \mathrm{f}=0.1 \mathrm{mHz}, \mathrm{E}_{\phi 0, \mathrm{k}}=0.13 \mathrm{mV} / \mathrm{m}$ and $\mathrm{m}=2$. 
In both cases the sector width is $\Delta \phi_{\mathrm{sec}}=\pi / 2$ with either one or four sectors in total. In each simulation, electrons have the initial conditions of $\mathrm{L}_{0}=4.6$, uniform distribution in MLT and $\mathrm{W}_{0}=0.1-4.0 \mathrm{MeV}$, giving a drift frequency range of $\mathrm{f}_{\mathrm{d} 0}=0.2-4.0 \mathrm{mHz}$. Like before, a set of 10 simulations with $\tau_{\text {sim }}=2 \mathrm{~h}$ are conducted and averaged together for different sets of initial, random phases in each one case. For the variance method the drift frequencies are set to $f_{d, k}=f_{k}+\Delta f / 2$ to avoid magnitude errors in the calculated $\mathrm{D}_{\mathrm{LL}}$.

Case a) demonstrates non-uniform diffusion coefficient as a function of drift frequency. The $D_{L L}$ coefficient obtained from the particle simulations coincide with the result from the variance method in equation (5.3.8) reasonably well as all values remain within the error bars (figure 5-5, left). However, the analytical approach is unable to reproduce the distinct plateau trends. The reason is because all phase information is ignored for each drift orbit and the integrations do not take into account any such resonance conditions. The non-uniform $\mathrm{D}_{\mathrm{LL}}$ profile is the result from the wave field exposure duration during each sector pass using the integration limit in (5.3.13). Since $\mathrm{D}_{\mathrm{LL}}$ fits within the error margin between the two methods used here, it is argued that the wave field exposure duration, derived from the sector width combined with the drift frequency, causes the non-uniformity in $\mathrm{D}_{\mathrm{LL}}$. 

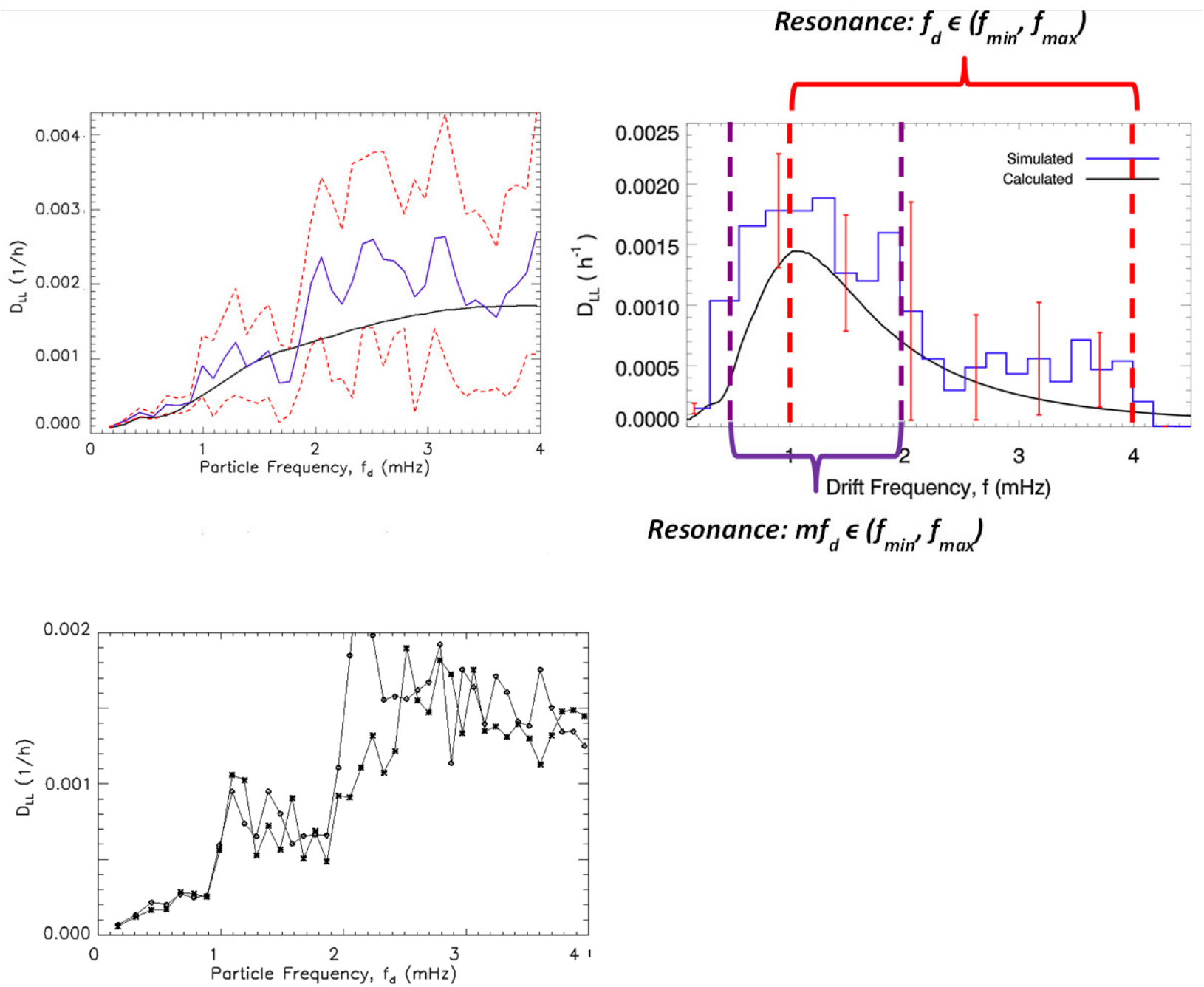

Figure 5-5: Comparison of simulated and analytically modeled $D_{L L}$ for cases a) and b). Resonant interval marked in red is for $f_{1} \leq f_{d} \leq f_{k}$, and the interval marked in purple is for $f_{1} \leq m f_{d} \leq f_{k}$.

Bottom: Simulated $D_{L L}$ using sectors with broadband spectra of $f_{k}=2-10 \mathrm{mHz}$. There is either one or four sectors of width $\Delta \phi_{\text {sec }}=\pi / 2 . D_{L L, 1 s e c}$ is multiplied by a factor of 4 to compensate the shorter time integral. Error bars are not shown. 
Figure 5-5 (right) shows the result from simulations and variance method for case b). Again $D_{L L}$ is not uniformly strong for different $f_{d}$. Since $|m|=2$, one must distinguish between two different resonant conditions that appear: $f_{d} \sim f_{k}$ and $\mathrm{mf}_{\mathrm{d}} \sim \mathrm{f}_{\mathrm{k}}$. The first type of resonance as described here considers the recurrence of phases for each drift orbit, being tied to the use of azimuthal sectors. Wavenumbers do not matter in the sense of this recurrence - only the actual drift frequency by itself. The second type of resonance is due to the spatial wave structure where particles remain fixed in the same phase along subsequent nodes in the azimuthal path. In the particle simulations the diffusion drops to negligible values once the drift frequency becomes larger than the highest frequency of the spectrum, i.e. $f_{d}>f_{K}$. Unfortunately the variance method fails to account for this phenomenon, thus another decision is to not consider those frequencies for the $\mathrm{D}_{\mathrm{LL}}$ evaluations. The diffusion coefficient does not drop to negligible values for $f_{d}<f_{1}$ here because those electrons still are within a subharmonic frequency band and still experience resonant scattering.

Another question is how strong the diffusive scattering would be when multiple MLT sectors, containing wave fields independent of each other, are assumed in the system. For case a) one set of particle simulations uses 4 sectors of wave activity spanning $\Delta \phi_{\mathrm{sec}}=\pi / 2$ each. The choices of width and number of sectors here make them span the entire azimuthal range where there are no spaces left without any wave activity. The reference simulation set, as already discussed for the top left figure, has only 1 such sector instead of 4 , where the diffusion is given a normalization multiplier of 4 is given for the sole sector to match the total wave power in both cases for the electrons drifting through these sectors. Figure 5-5 (bottom) shows the simulation results. The main thing to note here is that $D_{L L}, 4 \sec \sim 4 x D_{L L, 1 s e c}$ for all drift frequency except at 2.1-2.4 $\mathrm{mHz}$, thus each sector has an independent effect on the particle 
scattering. As long as the radial displacement, i.e. change in drift frequency, is negligible within each drift orbit this still holds true.

Since the variance technique has been demonstrated to match $D_{L L}$ values also found through particle simulations, this study will continue mapping spectral and spatial parameters in the system versus the diffusion rate employing variance calculations alone. The discussion in this section will cover the relation between sector widths, wavenumbers and frequencies versus the diffusion coefficient. In sections 5.6 and 5.7 two other parameters, initial L-shell placement and spectral index, are also discussed, but in this section those are considered to be fixed at $L=6$ and $\beta=0$ in all calculations.

\subsection{2 $D_{L L}$ as a function of MLT sector width}

One parameter associated with MLT sectors is the azimuthal width, for which we will investigate the diffusion coefficient function of in this section. We return to a straightforward case similar to the case in section 5.4 , where a monochromatic oscillation of $\mathrm{m}=0$, here set at $\mathrm{f}_{1}=8.0 \mathrm{mHz}$, exists within an MLT sector. We use the variance technique to evaluate $\mathrm{D}_{\mathrm{LL}}$ as function of $\Delta \phi_{\mathrm{sec}}$ with a particle population having drift frequencies $\mathrm{f}_{\mathrm{d}}=3-11 \mathrm{mHz}$.

Along with the width of the MLT sector particles do become exposed to different total wave power through each drift orbit. To eliminate this factor, we apply a normalization factor to the final diffusion coefficient:

$$
D_{L L, n}^{\text {norm }}=\frac{\Delta \phi_{\mathrm{sec}, 0}}{\Delta \phi_{\mathrm{sec}, n}} D_{L L, n}
$$


where $\mathrm{n}$ is the sector index. We show both the normalized and original $\mathrm{D}_{\mathrm{LL}}$ in figure 5-6.
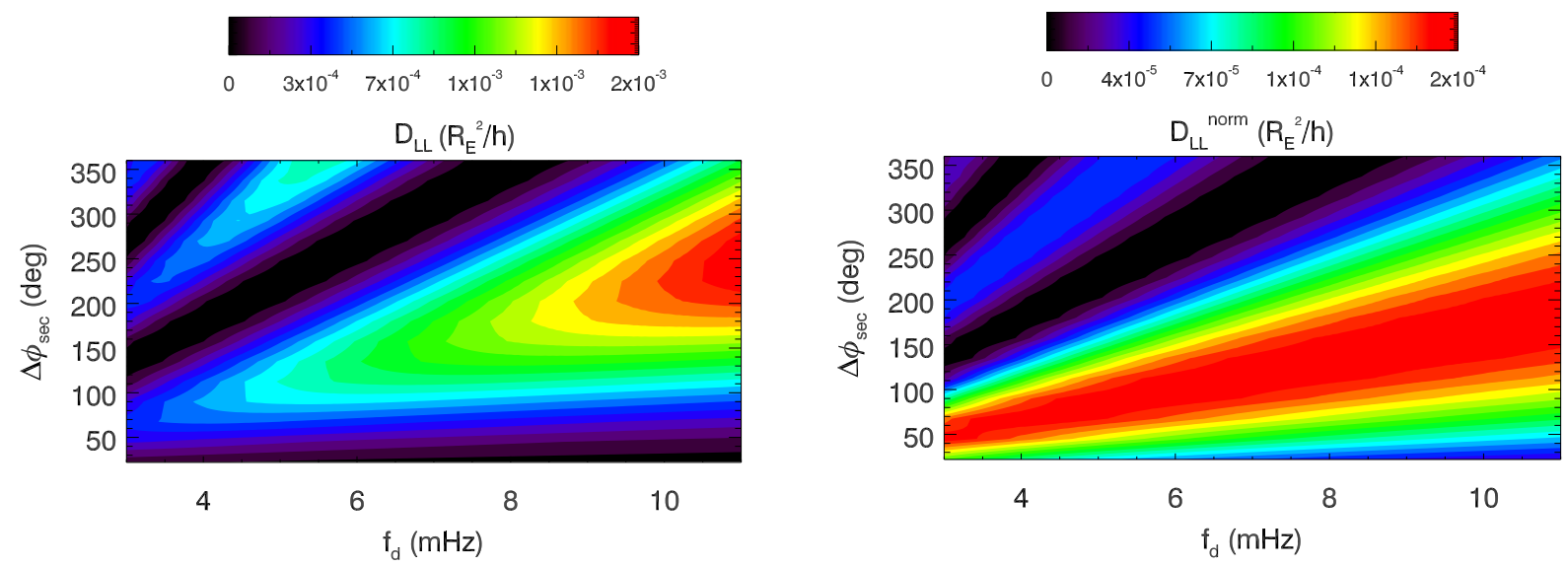

Figure 5-6: Variance method of $D_{L L}$ via eq. (5.3.12) for monochromatic oscillation of $f=8$ $m H z$ with $m=0$ confined in one azimuthal sector of varying widths. Left: Unnormalized $D_{L L}$, Right: Normalized $D_{L L}$ by equation (5.5.1).

As expected, the result is consistent with the discussion in section 5.4; the maximum diffusion coefficients occur when $\mathrm{f}_{\mathrm{r}} / \mathrm{f}_{\mathrm{k}}=2.63$. We rewrite the relation into:

$$
\left.f_{d}\right|_{\max \left(D_{L L}\right)}=2.63 f_{k} \frac{\Delta \phi_{\mathrm{sec}}}{2 \pi}
$$

For example, we find that particles drifting at $\mathrm{f}_{\mathrm{d}}=8.0 \mathrm{mHz}$ experience a maximum scattering when passing through a sector of width $\Delta \varphi_{\mathrm{sec}}=135 \mathrm{deg}$, which gives $\left.\mathrm{f}_{\mathrm{d}}\right|_{\max (\mathrm{DLL})}=2.63\left(\Delta \phi_{\mathrm{sec}} / 360\right) \mathrm{f}_{1}=2.63(135 / 360) \cdot 8 \mathrm{mHz}=7.9 \mathrm{mHz}$, thus a good match. Another example is at $\mathrm{f}_{\mathrm{d}}=4.0 \mathrm{mHz}$ where the a maximum occurs for $\Delta \phi_{\mathrm{sec}}=68 \mathrm{deg}$, which leads to $2.63(68 / 360) \cdot 8 \mathrm{mHz}=4.0 \mathrm{mHz}$. A higher harmonic is also visible above the main band in the contour plot. 
A problem that is clearly visible here is that $D_{L L}$ should approach zero for any $f_{d}$ as $\Delta \phi_{\text {sec }}$ approaches $2 \pi$. In reality when a global oscillation with a wavenumber of $m=0$ undergoes phase resets, there cannot be any net diffusive scattering of particles since there is no spatial reference phase to the oscillation. The variance method produces a $D_{L L}$ that assumes phase resets at every drift orbit, thus becomes non-zero even for $\Delta \phi_{\mathrm{sec}}=360 \mathrm{deg}$. For the purpose of understanding the diffusion coefficient function of drift frequencies and sector widths it still is a useful method however.

\subsection{3 $D_{L L}$ as a function of wave numbers}

Another main parameter to consider is the wavenumber. Since there can be MLT sectors at different widths, a question is how the wavenumbers should be defined. Up to this point the wavenumbers have been defined with the full range of magnetic local times $(0-24 \mathrm{~h})$, i.e. global m. An MLT sector can cover any arbitrary width in MLT and thus we can convert the global wavenumber into a local one:

$$
m_{\mathrm{sec}}=\frac{\Delta \phi_{\mathrm{sec}}}{2 \pi} m
$$

Again we maintain a simple system to begin with by assuming a monochromatic mode in an MLT sector at $\mathrm{f}_{1}=8 \mathrm{mHz}$. There are now two parameters that should be examined simultaneously: $\mathrm{m}$ and $\Delta \phi_{\text {sec }}$. The selected high frequency allows us to study a large range of these parameters. We evaluate $D_{L L}$ with the variance technique (fig 5-7) as a function of each 
separately by either maintaining $|\mathrm{m}|$ or $\Delta \phi_{\mathrm{sec}}$ while assigning a range of values for the other parameter.
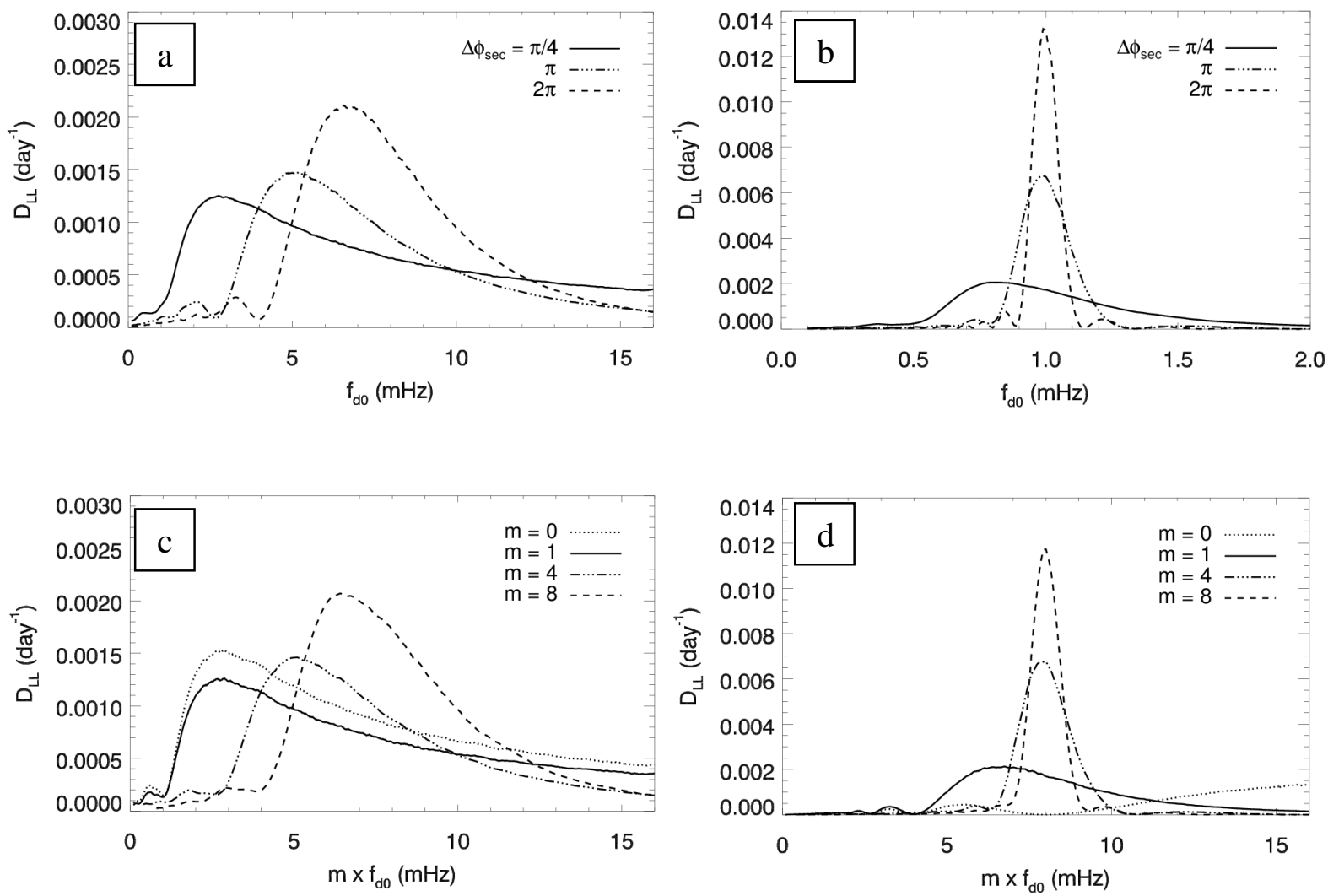

Figure 5-7: $D_{L L}$ as a function of wave number or sector width. A single wave field confined in the MLT sector has the frequency $f_{1}=8 \mathrm{mHz}$. The fixed parameters are: $\left.\left.\boldsymbol{a}\right) m=1, \boldsymbol{b}\right) m=8$, c) $\left.\Delta \phi_{\text {sec }}=\pi / 4, d\right) \Delta \phi_{s e c}=2 \pi$

The graphs in figures 5-7a-b are similar to fig. 5-7c-d, even in magnitude, which is due to equal values in $\mathrm{m}_{\mathrm{sec}}$ from equation (5.5.3). Thus as suggested, the solutions become functions of both $\mathrm{m}$ and $\Delta \phi_{\mathrm{sec}}$. Looking at figure $5-7 \mathrm{~b}$ it is clear that the width of the $\mathrm{D}_{\mathrm{LL}}$ function decreases as $\Delta \phi_{\text {sec }}$ increases; likewise for increasing $\mathrm{m}$ in figure 5-7d. A higher $\mathrm{m}_{\mathrm{sec}}$ leads to less net integrated wave power for non-resonant electrons drifting across the sector, thus the increased narrowness of $\mathrm{D}_{\mathrm{LL}}$ around the resonant frequency. The opposite occurs when 
$\mathrm{m}_{\mathrm{sec}}<1$ as in fig. 5-7a \& c, where instead non-resonant electrons gain relatively large $\mathrm{D}_{\mathrm{LL}}$ magnitudes compared to the resonant range, and a shift in the maximum farther apart from the resonant frequency $\mathrm{f}_{1}$. The profile for $\mathrm{m}=1$ is similar to the solution for $\mathrm{m}=0$ at $\Delta \phi_{\mathrm{sec}}=\pi / 4$ since the sector wave number has lost most of its effect on the diffusion, while for $\Delta \phi_{\mathrm{sec}}=2 \pi$ the difference between $\mathrm{m}=0$ and $\mathrm{m}=1$ is much larger.

We look more closely at the maximum $D_{L L}$ for $\left|m_{\text {sec }}\right| \Rightarrow>1$, which is a linear function of $m_{\text {sec }}$ and find it to be

$$
D_{L L, \max }=(5.7 \pm 0.8) \cdot 10^{-4}+(1.70 \pm 0.05) \cdot 10^{-3} m_{\mathrm{sec}} d a y^{-1}
$$

from combined values of $\mathrm{m}$ and $\Delta \phi_{\mathrm{sec}}$ (see figure 5-8). We can rewrite this relation by normalizing the coefficient to equation (5.3.11). Since $\mathrm{k}=1$ the function can be expressed as:

$$
D_{L L}=\frac{L^{6} E_{0}^{2}}{16 \xi^{2}(\beta) B_{z 0}^{2} R_{E}^{2}} \frac{1}{\tau_{r}} \frac{\psi_{s}\left(\omega_{d}, \tau_{r}\right)}{\omega_{1}^{\beta} \omega_{1}^{2}\left(1-m^{2} \omega_{d}^{2} / \omega_{1}^{2}\right)^{2}}
$$

where

$$
\begin{aligned}
& \psi_{s}\left(\omega_{d}, \tau_{r}\right)=6+\frac{2 m^{2} \omega_{d}^{2}}{\omega_{1}^{2}}+2\left(1-\frac{m^{2} \omega_{d}^{2}}{\omega_{1}^{2}}\right)\left[\cos \left(2 m \omega_{d} \tau_{r}\right)-\cos \left(2 \omega_{1} \tau_{r}\right)\right]- \\
& -\left(1+\frac{m \omega_{d}}{\omega_{1}}\right)^{2} \cos \left(2\left(\omega_{1}-m \omega_{d}\right) \tau_{r}\right)-\left(1-\frac{m \omega_{d}}{\omega_{1}}\right)^{2} \cos \left(2\left(\omega_{1}+m \omega_{d}\right) \tau_{r}\right)
\end{aligned}
$$

The fixed parameters compose the coefficient in (5.5.5) and turn the expression into:

$$
D_{L L}=\lambda \frac{1}{\tau_{r}} \frac{\psi_{s}\left(\omega_{d}, \tau_{r}\right)}{\left(1-m^{2} \omega_{d}^{2} / \omega_{1}^{2}\right)^{2}}
$$

where 


$$
\lambda=\frac{L^{6} E_{0}^{2}}{16 \xi^{2}(\beta) B_{0}^{2} R_{E}^{2} \omega_{1}^{2(1+\beta)}}
$$

Given the values used in this study $\left[\mathrm{B}_{0}=30500 \mathrm{nT}, \mathrm{R}_{\mathrm{E}}=6.8 \cdot 10^{6} \mathrm{~m}, \mathrm{E}_{0}=0.1 \mathrm{mV} / \mathrm{m}, \mathrm{L}=4.6\right.$, $\omega_{1}=2 \pi \cdot 8 \mathrm{mHz}, \beta=0$ ], the coefficient is $\lambda=8.51 \cdot 10-4$. Inserting this in (5.5.4) gives:

$$
D_{L L, \max }=(5.7 \pm 0.8) \cdot 10^{-4}+(2.00 \pm 0.06) \lambda m_{\mathrm{sec}} d a y^{-1}
$$

This linear function can simply be accounted for the linear increase in exposed wave power in the particle frame due to either increasing $\Delta \phi_{\text {sec }}$ or $\mathrm{m}$ - with slowed down particles instead. The special case is for $\mathrm{m}=0$, thus $\mathrm{m}_{\mathrm{sec}}=0$, which is not taken into account for this function.

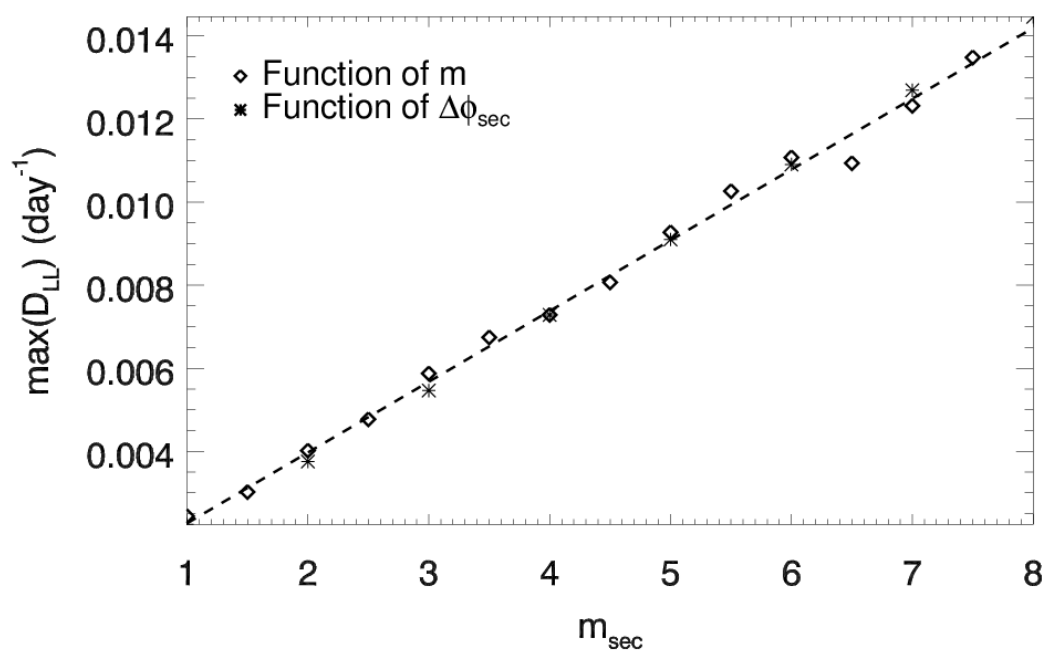

Figure 5-8: Maximum $D_{L L}$ as a function of $m_{\text {sec }}$, by either $m=8, \Delta \phi_{s e c}=\pi / 4-2 \pi$ - or $m=1-8$, $\Delta \phi_{s e c}=\pi$.

To complement this discussion we expand the wave field into a broadband spectrum. We set the frequencies to $f_{k}=4-8 \mathrm{mHz}$ while the particles drift at frequencies $0.1+\Delta f_{k} / 2 \leq f_{d, k} \leq 16.0+\Delta f_{k} / 2 \mathrm{mHz}$ with $\Delta f_{k}=0.01 \mathrm{mHz}$ to give an adequate coverage of 
the broadband spectrum. We repeat the same parameters as for the monochromatic waveparticle scattering and show the new $\mathrm{D}_{\mathrm{LL}}$ functions in fig 5-9.
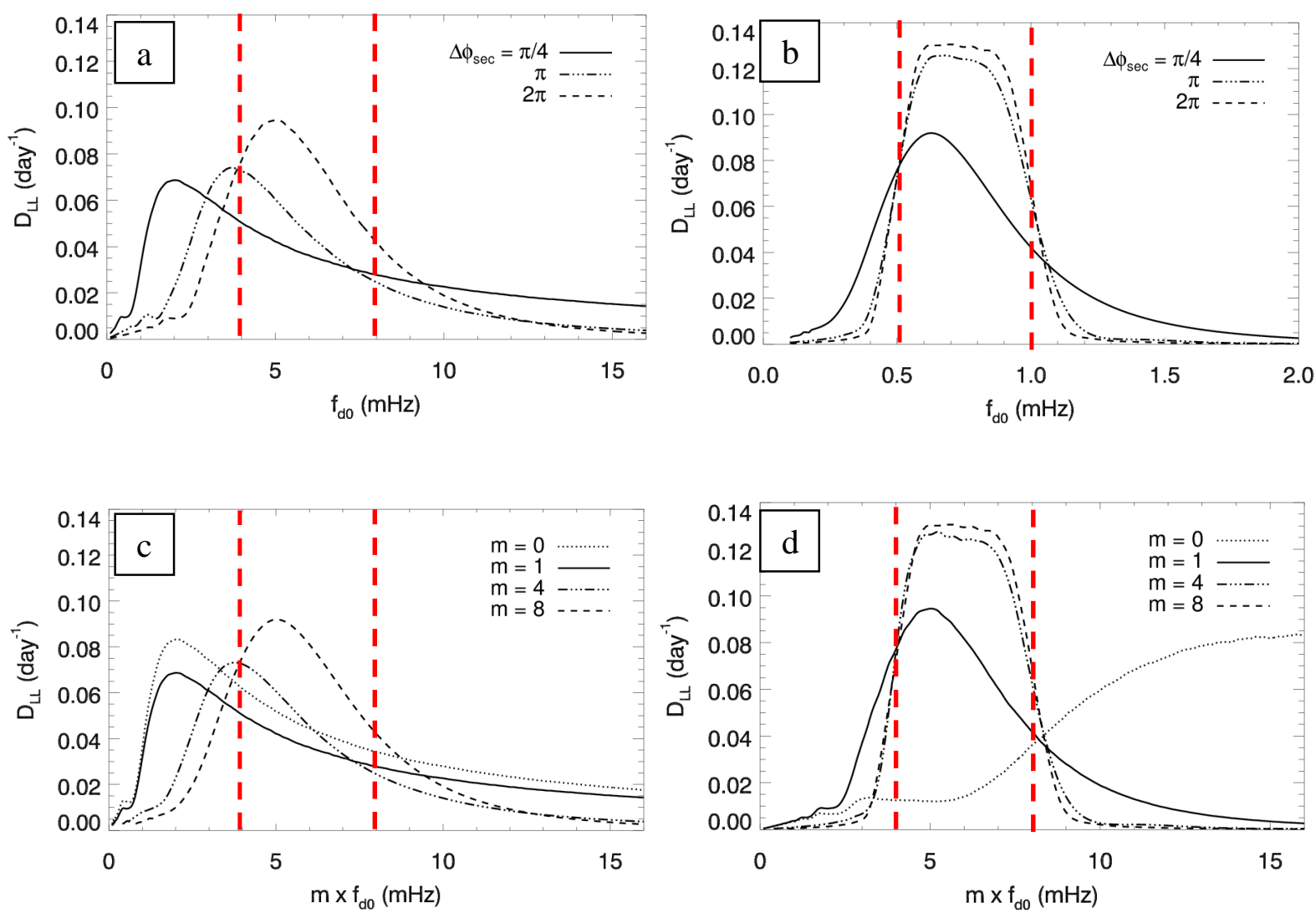

Figure 5-9: $D_{L L}$ as a function of wave number or sector width. A broadband wave field confined in the MLT sector has the frequency $f_{1}=4-8 \mathrm{mHz}$. The fixed parameters are: a) $\left.m=1, \boldsymbol{b}) m=8, \boldsymbol{c}) \Delta \phi_{\mathrm{sec}}=\pi / 4=6 \mathrm{~h}, \boldsymbol{d}\right) \Delta \phi_{\mathrm{sec}}=2 \pi=24 \mathrm{~h}$.

It becomes clear that the diffusion coefficient scales increasingly better with the uniform power spectral density, centered within the fundamental resonant band, as $\Delta \phi_{\text {sec }}$ increases. The scattering width for each mode becomes narrower in frequency as $\mathrm{m}_{\mathrm{sec}}$ increases, just like in fig $5-8 b \& d$, which explains the flatter profiles. 
For $m=0$, the frequencies where the maxima occur can be calculated with a similar procedure as Eq. (5.5.2), with the difference that one uses the average of all modes. For example, when $\Delta \phi_{\mathrm{sec}}=\pi / 4$ and $\mathrm{m}=0$ the maximum occurs at:

$$
\left.f_{d}\right|_{\max \left(D_{L L}\right)}=2.63 \frac{\left(f_{1}+f_{N_{k}}\right)}{2} \frac{\Delta \phi_{\mathrm{sec}}}{2 \pi}=2.63 \frac{(4+8)}{2} \frac{\pi / 4}{2 \pi} m \mathrm{~Hz}=1.98 \mathrm{mHz}
$$

which agrees well with the value $\left.f_{d}\right|_{\max \left(D_{L L}\right)}=2.06 \mathrm{mHz}$ found in the $\mathrm{D}_{\mathrm{LL}}$ function in figure 59c. However, it is non-trivial to find the optimal drift frequency for $\mathrm{m} \neq 0$ since the wave numbers contribute to $\mathrm{D}_{\mathrm{LL}}$ alongside $\tau_{\mathrm{r}}$ in equation (5.3.11). The centering and flattening of the $\mathrm{D}_{\mathrm{LL}}$ function with increasing $\mathrm{m}_{\mathrm{sec}}$ is the transition from non-resonant scattering via phase resets, to resonant scattering due to precise frequencies. For $\mathrm{m}_{\mathrm{sec}}=1.0 \pm 0.25$ we find $\left.f_{d}\right|_{\max \left(D_{L L}\right)}=6.91 \pm 0.57 \mathrm{mHz}$, or an effect of $\left(f_{1}-6.91\right) /\left(f_{1}-2.06\right) \cdot 100 \%=1.09 / 5.94 \cdot 100 \%=18.4 \%$ compared to the case for $\mathrm{m}_{\mathrm{sec}}=0$.
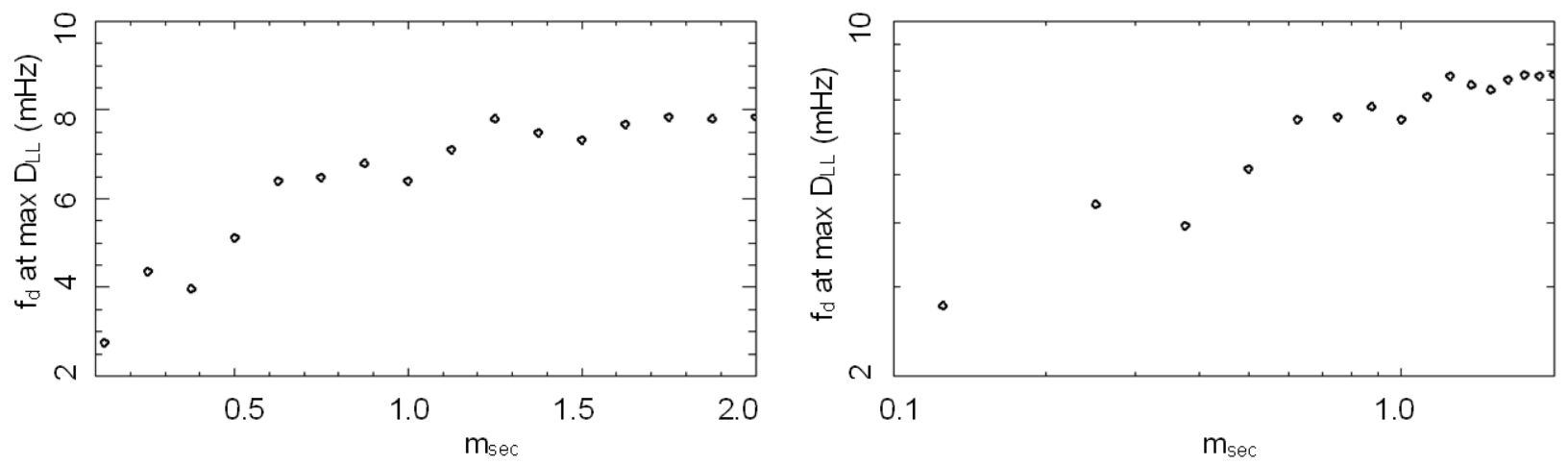

Figure 5-10: Drift frequencies at maximum $D_{L L}$ for $f_{1}=8 \mathrm{mHz}, \Delta \phi_{s e c}=\pi / 4$ and $\mathrm{m}=0-16$. 


\section{6 $\mathrm{D}_{\mathrm{LL}}$ as a Function of Power-law Spectral Indices}

\subsubsection{Case: Global oscillations}

Up to this point every spectrum has been considered with uniform amplitudes for each mode ( $\beta=0$ in (5.3.5) ). Observations of magnetospheric data have shown the existence of frequency dependent powers of wave fields in both the solar wind and the magnetosphere. Typically the properties of the solar wind spectra consist of negative spectral indices $\beta$ with strong mode amplitudes at low frequencies [Bloom and Singer, 1995], which are also mirrored in the magnetospheric response wave fields.

We return to the case for global wave field constructed by randomizing phases - similar to the examples given in section 5.4 , but with the addition of a non-zero $\beta$. No sectors are used for this demonstration, thus positions of particles can be ignored. The spectrum covers $1 \mathrm{mHz} \leq \mathrm{f}_{\mathrm{k}} \leq 0.3 \mathrm{~Hz}$. The reset frequency is $\mathrm{f}_{\mathrm{r}}=1 \mathrm{mHz}$ - the lowest frequency in the broadband spectrum, in order to ensure a diffusive scattering of particles at a steady rate due to extrinsic stochasticity. We assign power law values $-0.8 \leq \beta \leq 2.8$. The simulation population consists of 200 electrons launched with equal initial conditions $\mathrm{W}_{0}=2 \mathrm{MeV}$ and $\mathrm{L}_{0}=4.6$. Since there is no spatial reference, we assign each electron its own unique time series of phase resets to ensure diffusion. See figure 5-11 for the simulated diffusion coefficients.

The diffusion coefficient dependence on $\beta$ slowly decreases for $-0.8<\beta<0.0$, and then starts to increase rapidly as $\beta$ becomes positive, with a saturation at about $\beta=1.6$. As comparison 
we calculate the electric field amplitude for the lowest and the highest frequencies, i.e. $E_{\phi, \text { amp }}\left(f_{1}\right)=\frac{E_{0,1}}{\xi(\beta) f_{1}^{\beta}}$ and $E_{\phi, \text { amp }}\left(f_{N_{k}}\right)=\frac{E_{0, N_{k}}}{\xi(\beta) f_{N_{k}}^{\beta}}$. We note that the diffusion rates match fairly well with respective $\mathrm{E}_{\phi, \mathrm{amp}}-\mathrm{E}_{\phi \text {,amp }}\left(\mathrm{f}_{1}\right)$ with $\beta>0$, and $\mathrm{E}_{\phi \text {,amp }}\left(\mathrm{f}_{2}\right)$ with $\beta<0$. This is consistent with the general theory (Eq. (5.3.4) ) that the diffusion rate is proportional to the local power spectral density of the spectrum. By making the low-frequency modes stronger in amplitude $(\beta>0)$, with the mode at $f_{1}$ the strongest, $D_{L L}$ will be dominated by those, and vice versa for $\beta<0$.

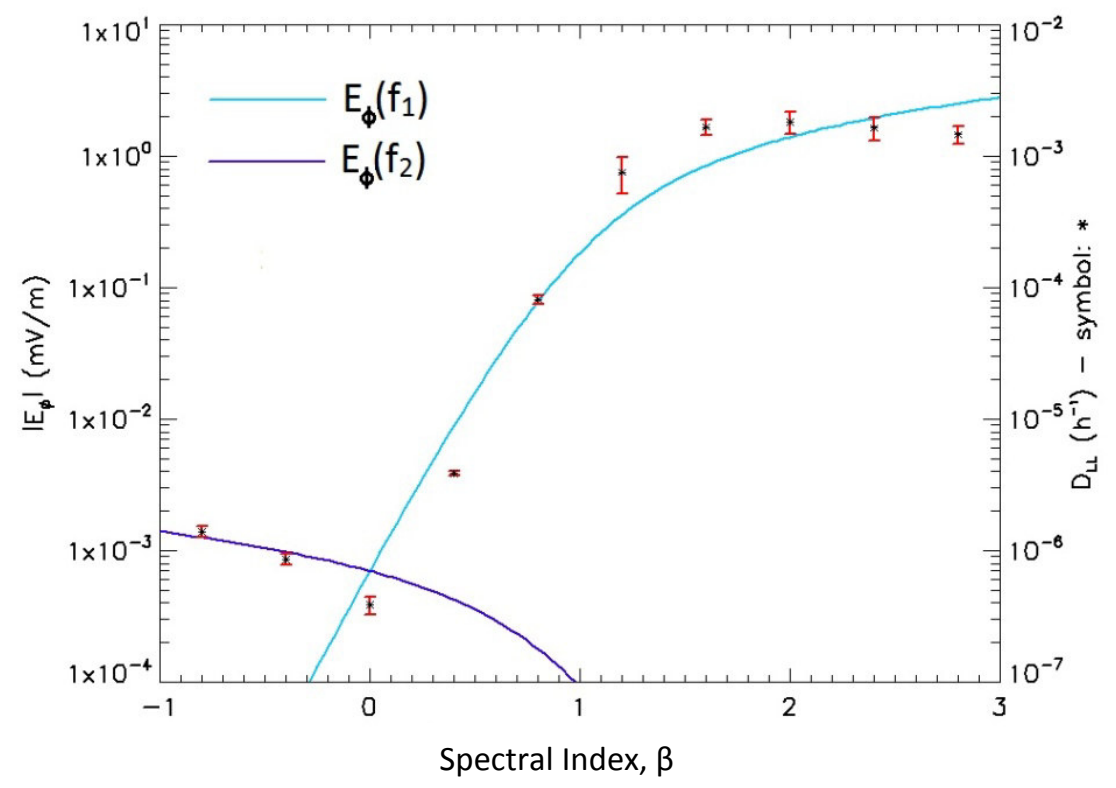

Figure 5-11: Electric field amplitudes at extremes of spectrum, and $D_{L L}$ versus spectral index in a global spectrum of oscillations. 


\subsubsection{Case: MLT sectors}

The next step is to introduce spatial dependency once again as a parameter for phase resets. For this purpose, we consider MLT sector widths and wavenumbers in the models. As before, it is straightforward to start with $\mathrm{m}=0$, for which case figure 5-12 gives the outlined evaluations for $D_{L L}$. We assign wave frequencies $f_{k}=2-5 \mathrm{mHz}$ and assume two different sector widths of $\Delta \phi_{\mathrm{sec}}=\pi / 2$ and $3 \pi / 2$ for the model. The integration in the variance calculation is over a partial oscillation for any frequency, where the integration limit is $\tau_{r}=\frac{\Delta \phi_{\mathrm{sec}}}{2 \pi / T_{d}}=\frac{\pi / 2}{2 \pi} T_{d}=\frac{T_{d}}{4}$

The smaller sector width, $\Delta \phi_{\mathrm{sec}}=\pi / 2$, gives a result in good agreement with the peak $\mathrm{D}_{\mathrm{LL}}$ in fig. 5-12, being concentrated at the lowest frequency for higher spectral indices. This can be checked with equation (5.5.10) while we consider the flat spectrum $\beta=0$. The result $f_{d}=1.64 \mathrm{mHz}$ matches well with the variance calculations.

For $\beta>0$ a weight function, normalized to the lowest frequency $\mathrm{f}_{1}$, is needed to calculate for each drift frequency. It is defined via the numerator of $f_{k}^{2 \beta}$ that decreases the effect on diffusion from each mode with increasing frequency. Setting the reference mode at the lowest point, $\mathrm{f}_{1}$, the weight function is

$$
w_{k}=\left(\frac{f_{1}}{f_{k}}\right)^{2 \beta}
$$


The maximum $\mathrm{D}_{\mathrm{LL}}$ is then found at:

$$
\left.f_{d}\right|_{\max \left(D_{L L}\right)}=2.63 \frac{\Delta \phi_{\mathrm{sec}}}{2 \pi} \frac{\sum_{k=1}^{N_{k}} w_{k} f_{k}}{\sum_{k=1}^{N_{k}} w_{k}}
$$

Using this equation for $\beta=1.5$ the sector width $\Delta \phi_{\mathrm{sec}}=\pi / 2$ causes a maximal $\mathrm{D}_{\mathrm{LL}}$ at $\mathrm{f}_{\mathrm{d}}=0.91$ $\mathrm{mHz}$, and $\Delta \phi_{\mathrm{sec}}=3 \pi / 2$ gives $\mathrm{f}_{\mathrm{d}}=2.74 \mathrm{mHz}$. The corresponding values found from the variance method in figure 5-12 are $\mathrm{f}_{\mathrm{d}}=1.10 \mathrm{mHz}$ for $\Delta \phi_{\mathrm{sec}}=\pi / 2$, and $\mathrm{f}_{\mathrm{d}}=2.80 \mathrm{mHz}$ for $\Delta \phi_{\mathrm{sec}}=3 \pi / 2$. The basic picture is that the maximum $\mathrm{D}_{\mathrm{LL}}$ shifts toward particles of higher drift frequencies for an increasing sector width when the spectral index has a positive value.

This is repeated for a non-zero wavenumber, $|\mathrm{m}|=1$, for the same spectrum (fig. 5-13). A dependence on $\Delta \varphi_{\text {sec }}$ is still discerned for the peak $\mathrm{D}_{\mathrm{LL}}$, but not as widely separated as for $\mathrm{m}=$ 0. Again as discussed at the end of section 5.5.3, the power spectral density at local frequencies centers the $\mathrm{D}_{\mathrm{LL}}$ profile within the resonant band. 

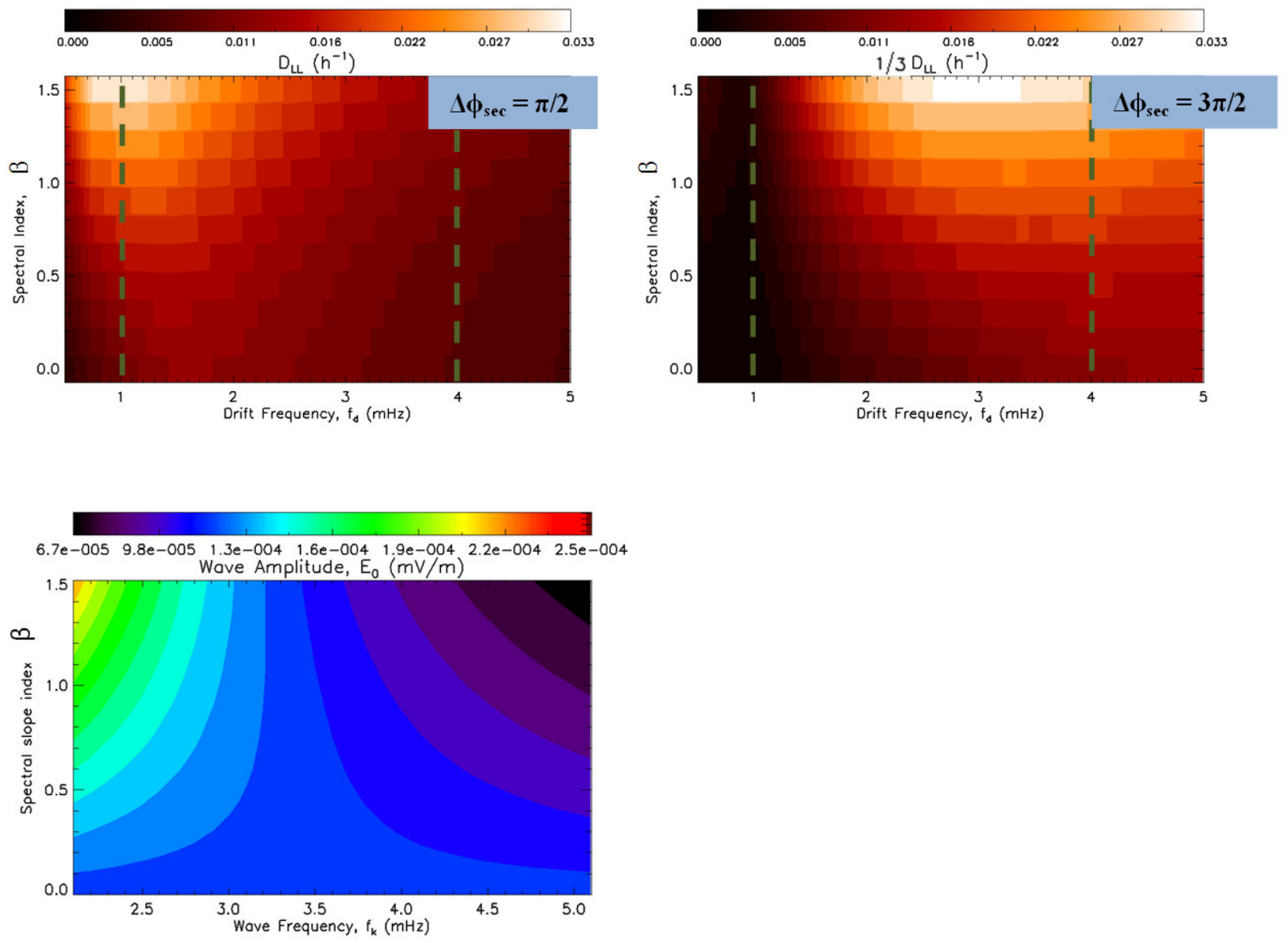

Figure 5-12: Top: $D_{L L}$ for a broadband spectrum of $f_{k}=1-4 \mathrm{mHz}, \mathrm{m}=0$, evaluated for various drift frequencies, spectral indices and sector widths. Bottom: Wave electric field amplitude profile versus frequencies and spectral indices.

A modeling concern regarding the assumptions of non-zero $\beta$ is the possibility of particle scattering becoming more sensitive to local frequencies since the mode amplitudes are nonuniform. The variance method may encounter an issue in accuracy since it assumes fixed Lshells, i.e. drift frequencies, while outside our model the radial position shifts as the electrons interact with waves. To complement the results discussed up to this point we provide a set of simulations as well (figure 5-14) in the same manner as before (10 sets, 100 electrons in each with one initial $\mathrm{f}_{\mathrm{d}}$, one initial L-shell and uniformly distributed MLT placements). A global 
$\left(\Delta \phi_{\mathrm{sec}}=2 \pi\right)$ electric field spectrum with the same wavenumber $|\mathrm{m}|=2$ and the same amplitude $\mathrm{E}_{0}=0.1 \mathrm{mV} / \mathrm{m}$ as the initial case in section 5.2.
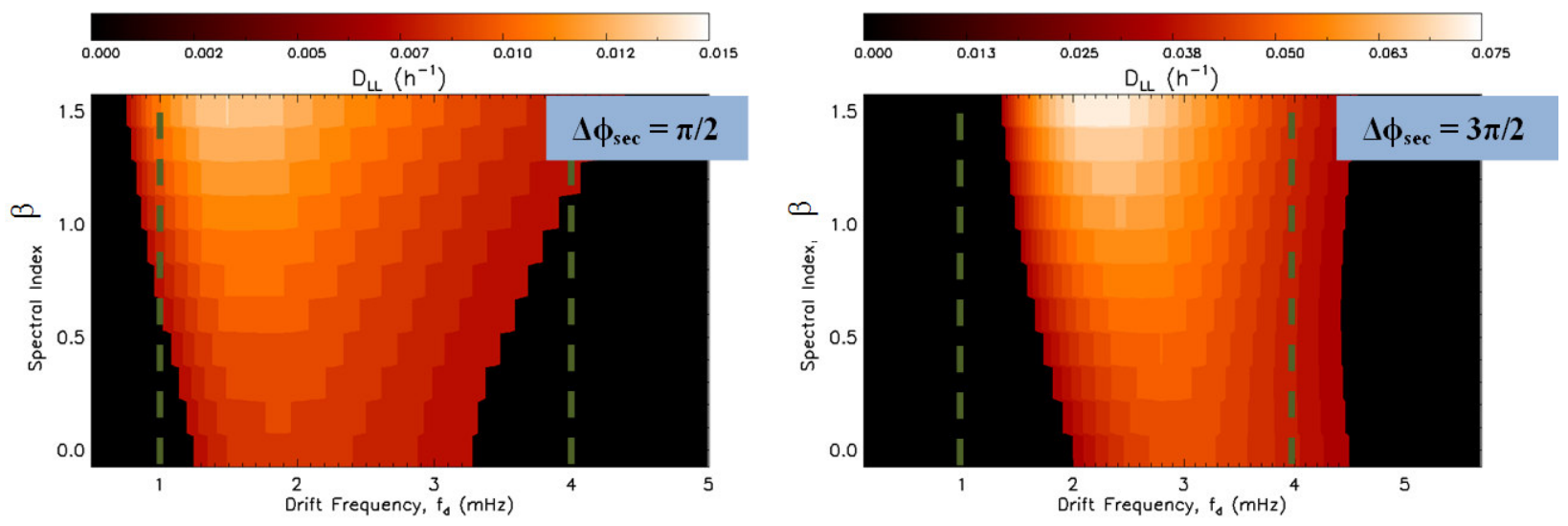

Figure 5-13: As in fig. 5-12 top, with $|m|=1$.

The diffusion rate remains within the same range as for $\beta=0$. It is also interesting that the mean radial positions have approximately the same scatter width in both cases. Electrons scattered towards higher frequencies encounter smaller amplitudes, and vice versa for lower frequencies. In addition, the L-shell dependence for $D_{L L}$ should amplify this effect further. Regardless, the diffusion rate remains the same at these amplitudes, thus the variance method is still a valid model. 

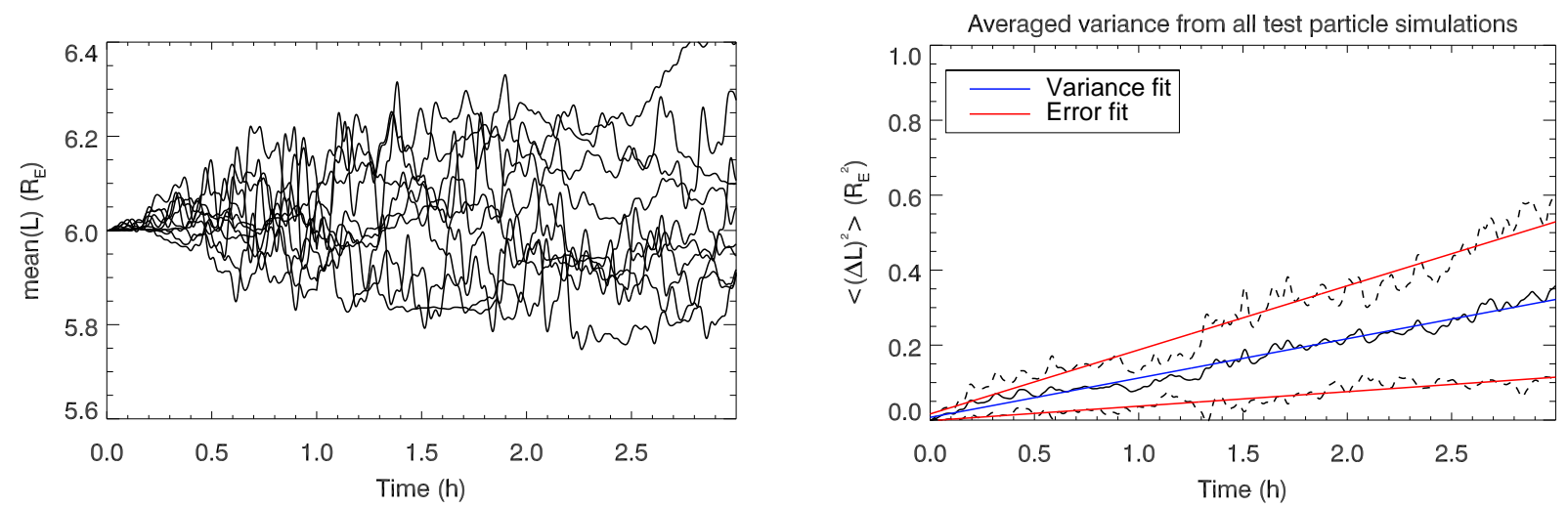

Figure 5-14: Variance evolution of radial position due to broadband spectra of random phases, $f_{k}=1-10 \mathrm{mHz}$. Left: Mean value of radial locations. Right: Averaged variance from 10 simulations.

\subsection{Radial Dependence of $\mathrm{D}_{\mathrm{LL}}$}

We have investigated the effect drift frequency and MLT sectors widths have on the diffusion rate in this chapter. Another parameter that is of importance in understanding radiation belt dynamics is L-shell position of the particles. In diffusion theory, this parameter is pointed out in for example equation (5.3.4) where in our case $\mathrm{D}_{\mathrm{LL}} \alpha \mathrm{L}^{6}$. However this proportionality must also be adjusted due to the fact that $f_{d} \alpha$ L from equation (2.1.16). Therefore this section will parameterize the effect on $D_{L L}$ by L-shell, $f_{d}$ and $\Delta \phi_{\text {sec }}$ simultaneously through the application of the variance method. We generate waves with a broadband spectrum of $\mathrm{f}_{\mathrm{k}}=4-8 \mathrm{mHz}$ and initialize the electrons at the energy $\mathrm{W}_{0}=1 \mathrm{MeV}$, making $\mathrm{f}_{\mathrm{d}}$ as a function of $\mathrm{L}_{0}$. The resulting diffusion coefficients $D_{\text {LL }}$ are power law functions of the L-shells, which indices are given in table 4-1 below. 


\begin{tabular}{|c|c|c|c|c|}
\hline Wavenumber & L-shells & $\begin{array}{c}\text { Drift } \\
\text { Frequencies } \\
(\mathrm{mHz})\end{array}$ & Sector Width & $\mathrm{D}_{\mathrm{LL}} \alpha$ \\
\hline $\mathrm{m}=0$ & $8.4-16.0$ & $3.9-7.5$ & $\pi / 2$ & $\mathrm{~L}^{4.56 \pm 0.02}$ \\
\hline$\|$ & $\|$ & $\|$ & $\pi$ & $\mathrm{L}^{6.36 \pm 0.10}$ \\
\hline$\|$ & $\|$ & $\|$ & $3 \pi / 2$ & $\mathrm{~L}^{7.85 \pm 0.08}$ \\
\hline $\mathrm{m}=1$ & $8.4-16.0$ & $3.9-7.5$ & $\pi$ & $\mathrm{L}^{5.25 \pm 0.05}$ \\
\hline $\mathrm{m}=2$ & $4.6-8.4$ & $2.0-3.8$ & $\pi / 2$ & $\mathrm{~L}^{5.08 \pm 0.04}$ \\
\hline$\|$ & $4.6-6.8$ & $2.0-3.2$ & $\|$ & $\mathrm{L}^{5.32 \pm 0.05}$ \\
\hline$\|$ & $7.0-8.4$ & $3.3-3.8$ & $\|$ & $\mathrm{L}^{4.70 \pm 0.01}$ \\
\hline$\|$ & $4.6-8.4$ & $2.0-3.8$ & $\pi$ & $\mathrm{L}^{5.25 \pm 0.11}$ \\
\hline$\|$ & $4.6-6.8$ & $2.0-3.2$ & $\|$ & $\mathrm{L}^{5.90 \pm 0.11}$ \\
\hline$\|$ & $7.0-8.4$ & $3.3-3.8$ & $\|$ & $\mathrm{L}^{4.17 \pm 0.04}$ \\
\hline $\mathrm{m}=4$ & $2.3-4.2$ & $1.0-1.9$ & $\pi / 4$ & $L^{5.08 \pm 0.04}$ \\
\hline$\|$ & $\|$ & $\|$ & $\pi / 2$ & $\mathrm{~L}^{5.24 \pm 0.11}$ \\
\hline$\|$ & $\|$ & $\|$ & $\pi$ & $\mathrm{L}^{5.09 \pm 0.19}$ \\
\hline
\end{tabular}

Table. 4-1: $D_{L L}$ as a function of $L$ due to various wave- and environment parameters.

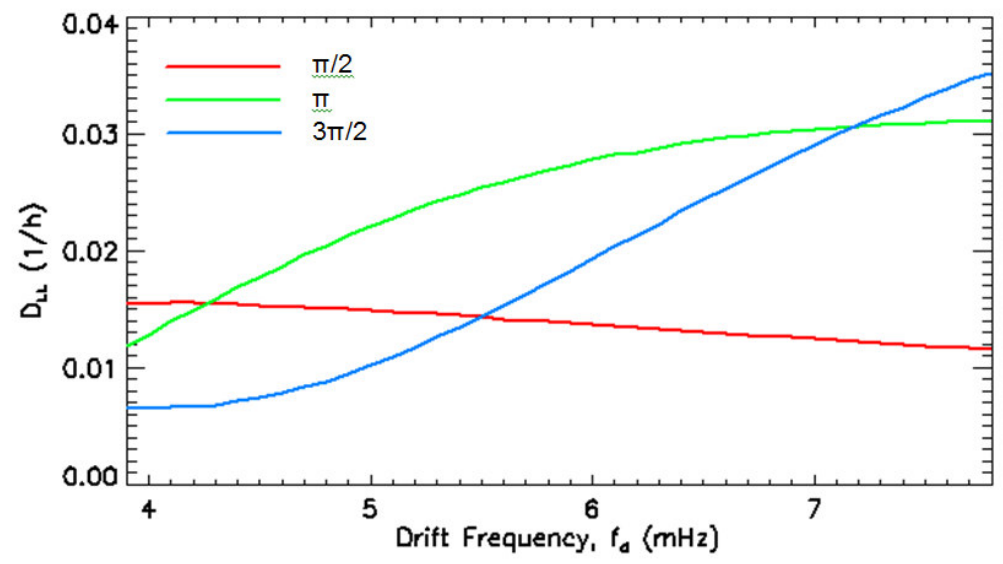

Figure 5-15: $D_{L L}$ vs drift frequency for $f_{k}=4-8 \mathrm{mHz}, m=0, \Delta \phi_{s e c}=\pi / 2, \pi$ and $3 \pi / 2$. 
For $|m| \geq 1$ and the data given in table 5-1 the $\mathrm{D}_{\mathrm{LL}}$ function of L-shell is $\mathrm{L}^{5.11 \pm 0.44}$, where the standard deviation of the index is due to dependence on wavenumber and sector width. The diffusion function (5.3.11), in combination with equations (2.1.16) and (5.3.13), is

$$
D_{L L} \propto \frac{L^{6}}{\tau_{r}}=f_{d} L^{6} \propto L^{5}
$$

which explains the typical exponent index observed here.

The calculations for $|\mathrm{m}|=0$ vary by approximately $\mathrm{L}^{ \pm 3}$ for the 3 sector widths evaluated, which is due to a strong particle response to the sector exposure time $\tau_{\mathrm{r}}$ for each mode in the system. For $|\mathrm{m}| \neq 0$ the deviation in L-shell power law index varies less. For example, by finding the best fit to the drift frequencies divided into two separate ranges, on either side of the peak in figure 5-15, give two different indices. These are $\mathrm{L}^{5.32 \pm 0.05}$ for the low frequency range and $\mathrm{L}^{4.70 \pm 0.01}$ for the high frequency range, with exponential deviations of $5.32-5.08=0.24$ and 5.08-4.70 $=0.38$ apart from the average fitted trend respectively.

In parallel, we find a similar scaling for drift frequencies derived from assigning varying energies while letting the L-shell remain constant (see fig 5-16). For the same spectral parameters we find the best fit functions for the diffusion coefficient on both sides of the $\mathrm{D}_{\mathrm{LL}}$ peak, which are $D_{L L} \sim f_{d}^{0.66 \pm 0.05}$ for the low frequency range and $D_{L L} \sim f_{d}^{-0.42 \pm 0.01}$ for the high frequency range. The ratio of the absolute value between these two trends is $0.66 / 0.42=1.57$ while the ratio for the power-law deviations for the L-shell fits is $0.38 / 0.24=1.58$. Thus the L-shell dependency for the diffusion coefficient is directly responsive to the adjustments in drift frequencies as well. 


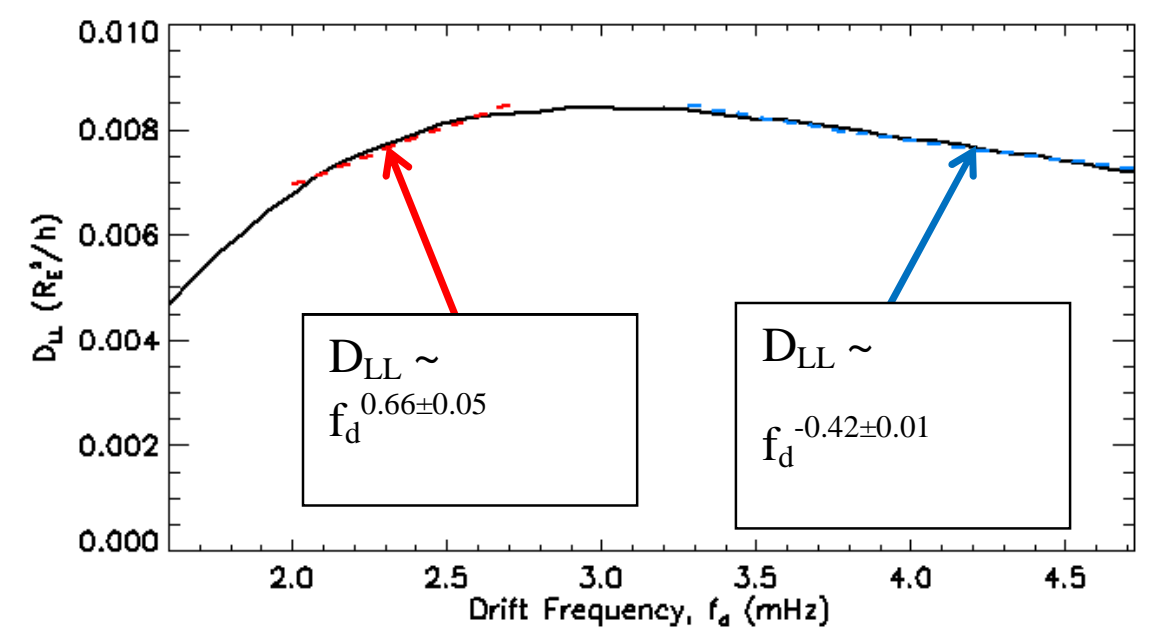

Figure 5-16: $D_{L L}$ vs drift frequency for $f_{k}=4-8 \mathrm{mHz}, m=2, \Delta \phi_{s e c}=\pi$.

Lastly, we conduct a set of test particle simulations for 4 sectors containing spectra of $\mathrm{f}_{\mathrm{k}}=3.8-5.8 \mathrm{mHz}, \mathrm{m}=0$ and the particles launched at $\mathrm{L}_{0}=3.0-7.0$ and $\mathrm{W}_{0}=3.0 \mathrm{MeV}$, which gives $\mathrm{f}_{\mathrm{d} 0}=2 \cdot 0-4.7 \mathrm{mHz}$. We chose these parameters here in order to cover the fundamental frequencies, as well as the first subharmonic frequencies - both separated by a gap. The resulting $D_{L L}$ magnitudes for each initial drift frequency are shown in figure 5-17. We create a best fit curve function on drift frequencies that are either in fundamental or subharmonic resonance: $\mathrm{D}_{\mathrm{LL}} \sim \mathrm{L}^{7.28 \pm 0.33}$ which is on the steeper side of the possible power-law scalings. With the help of equation (5.5.2), the peak $\mathrm{D}_{\mathrm{LL}}$ is expected to occur at:

$$
\left.f_{d}\right|_{\max \left(D_{L L}\right)}=2.63 \frac{\left(f_{1}+f_{N_{k}}\right)}{2} \frac{\Delta \phi_{\mathrm{sec}}}{2 \pi}=2.63 \frac{(3.8+5.8)}{2} \frac{\pi / 2}{2 \pi} m H z=3.16 \mathrm{mHz}
$$

which is the drift frequency for electrons launched beyond $L_{0}=7$. Thus $D_{L L}$ increases with $f_{d}$ for all the fundamental frequencies, thus contributing to an additional power law relation in excess of $\mathrm{D}_{\mathrm{LL}} \alpha \mathrm{L}^{6}$, since $D_{L L} \propto f_{d}^{\sigma}$ where $\sigma<0$. The profile matches well with both the fundamental as well as the $1^{\text {st }}$ subharmonic interactions, which diffusion values are far above 
the excluded gap with frequencies $f_{d}=2.9-3.8 \mathrm{mHz}$. The gap between the resonant harmonics has a much lower diffusion rate as well as an even steeper radial profile.

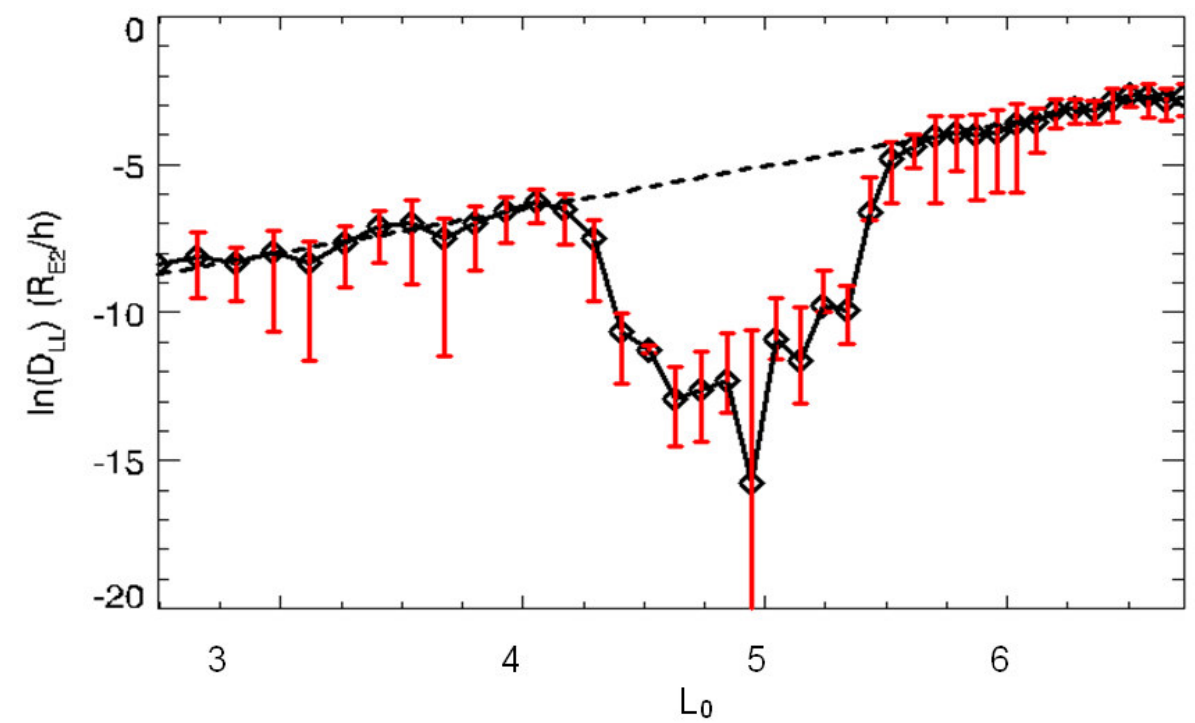

Figure 5-17: $D_{L L}$ from simulations with $f_{k}=3.8-5.8 \mathrm{mHz}, m=0 . L_{0}=3-7, W_{0}=3.0 \mathrm{MeV}$.

\subsection{Conclusions}

In this chapter we have explored effects of various parameters on the electron diffusion coefficient in ideal magnetospheric settings. We employed guiding-center simulations and a variance method integrating the wave fields directly. One fundamental assumption for the variance method is non-ergodicity, i.e. relative phases between waves and particles undergo resets within drift orbit time scales. This analytical approach fails to represent resonant scattering for narrowband spectra, but works well for broadband spectra with uniform amplitudes. 
Via both the variance method and test particle simulations we found that $\mathrm{D}_{\mathrm{LL}}$ is non-uniform as a function of frequency for flat power spectra $(\beta=0)$. Each mode in a broadband spectrum can contribute to electron scattering via phase resets, where a cumulative effect of nonresonant modes can add significantly to a final $\mathrm{D}_{\mathrm{LL}}$ profile. Thus it is not sufficient to only consider the local power spectral density as the main driver for $\mathrm{D}_{\mathrm{LL}}$. The phase resets can be either directly imposed at regular intervals, or indirectly caused by particle drift orbits passing through MLT sectors of strong ULF wave activity. While considering a spectrum of uniform amplitude, and that every mode in the spectrum has equal weight towards $\mathrm{D}_{\mathrm{LL}}$, we find a maximum $D_{L L}$ at the phase reset frequency of $f_{r}=2.63 f_{k}$, where $f_{k}$ is any selected mode for which the diffusion coefficient is calculated for. On the other hand, if the spectrum is a power-law function $(\beta \neq 0)$ the maximum can still be found but with corresponding weight coefficients on each mode ( Eq. (5.6.2) ). For increasing sector wave number $\mathrm{m}_{\text {sec }}$ we find that the $\mathrm{D}_{\mathrm{LL}}$ function rapidly approaches proportionality to the PSD at local frequencies.

Another important parameter in determining the diffusion rate is the L-shell position of particles where, in the base coefficient of the variance calculation, the relation for a wave $\mathrm{E}_{\phi}$ field in an azimuthally symmetric dipole is $D_{L L} \alpha L^{6}$. The introduction of azimuthal sectors requires an extra $f_{d}$ in this coefficient which reduces the power-law to $D_{L L} \alpha L^{5}$. This relation must also be adjusted due to additional local $f_{d}$ dependence in frequency space due to dynamic phases from sector passes. 


\section{Event Study for Pre Storm-Commencement on November 7, 2004}

This chapter continues the discussion in chapter 5 with a case demonstration in a realistic MHD field model for the pre storm-commencement on Nov 7, 2004. We show ULF wave features in relevant field components that are potentially capable of driving electron scattering in the magnetosphere. We use the spatial and spectral properties of the wave electric field to calculate the diffusion coefficients at each electron drift frequency. The diffusion requires a combination of power spectral density at local frequencies and phase resets to match with results from our test-particle simulation.

\subsection{Introduction}

We have discussed both short-term and long-term electron scattering in the outer belt during ideal conditions in chapters 4 and 5 respectively. This chapter will extend the ideal settings into a realistic scenario where the theories in wave-particle interactions and the techniques in evaluation will be put to test. The choice of event is on 2004/07/11 during which day a geomagnetic storm occurs, and there is associated ULF wave activity; both before and after the storm onset. We will investigate a time interval before the onset since the magnetosphere is at a relatively steady configuration, with wave fields that turn out to be stable for a sufficiently long period to evaluate wave-particle interactions. To determine realistic electromagnetic fields in the inner magnetosphere we use the Open Geospace General Circulation Model (OpenGGCM). It is a global three-dimensional resistive MHD model using 
solar wind parameter data sampled by the WIND satellite located outside the magnetosphere between Earth and the Sun. [ Raeder, 1998 ]

\subsection{The MHD Model}

For evaluating the electron scattering we extract the electromagnetic fields in the OpenGGCM from the magnetic equatorial plane in the GSM coordinate system. The source data is provided in Cartesian GSM coordinates from which we extract $-11<(\mathrm{x}, \mathrm{y})<11 \mathrm{R}_{\mathrm{E}}$ with spatial resolution $\Delta \mathrm{x}=\Delta \mathrm{y}=0.1 \mathrm{R}_{\mathrm{E}}$ and a temporal time step $\Delta \tau_{\text {grid }}=30 \mathrm{~s}$. The temporal resolution provides sufficient information to yield frequencies up to $f_{k}=8.3 \mathrm{mHz}$ with a spectral resolution $\Delta \mathrm{f}=0.17 \mathrm{mHz}$. It is convenient to transform the grid into cylindrical coordinates for this study where the radial resolution is $\Delta \mathrm{L}=0.1$ and the azimuthal resolution is $\Delta \phi=1.0^{\circ}$ or 4 minutes in MLT.

It must be noted that the OpenGGCM does not include a ring current nor a plasmasphere. The implication is that the wave fields can be non-reliable at L-shells normally covering these regions of particles - typically $\mathrm{L}<5$. Therefore, any wave-particle modeling is only conducted at the outer L-shells. 


\subsection{Event Description}

The event that begun on 2004/07/11 was a strong geomagnetic storm, caused by an interplanetary magnetic cloud that struck the magnetosphere at 18:36 UT. The Dst index was perturbed for several days after the storm onset on the $7^{\text {th }}$ (figure 6-1 bottom). ULF wave activities can be expected to occur within this interval, but it is not the main phase of the storm itself that is of interest in this study however. In order to test and understand the results found in this dissertation the conditions need to be relatively quiet, as opposed to a dynamic system throughout a main phase of a storm. It turns out that the pre-commencement stage offers a steady environment where waves also will be shown to exist. There is an interval within the onset between 17:00-18:40 UT when the solar wind data (figure 6-1 top left and right) show a tailward solarwind speed of large and steady values between $450-500 \mathrm{~km} / \mathrm{s}$, high

ion density at $10-20 \mathrm{~cm}^{-3}$ and a steadily northward IMF at 15-20 nT. This interval is suitable for testing the electron scattering due to wave-particle interaction due to its stationarity over a reasonably long time frame. 

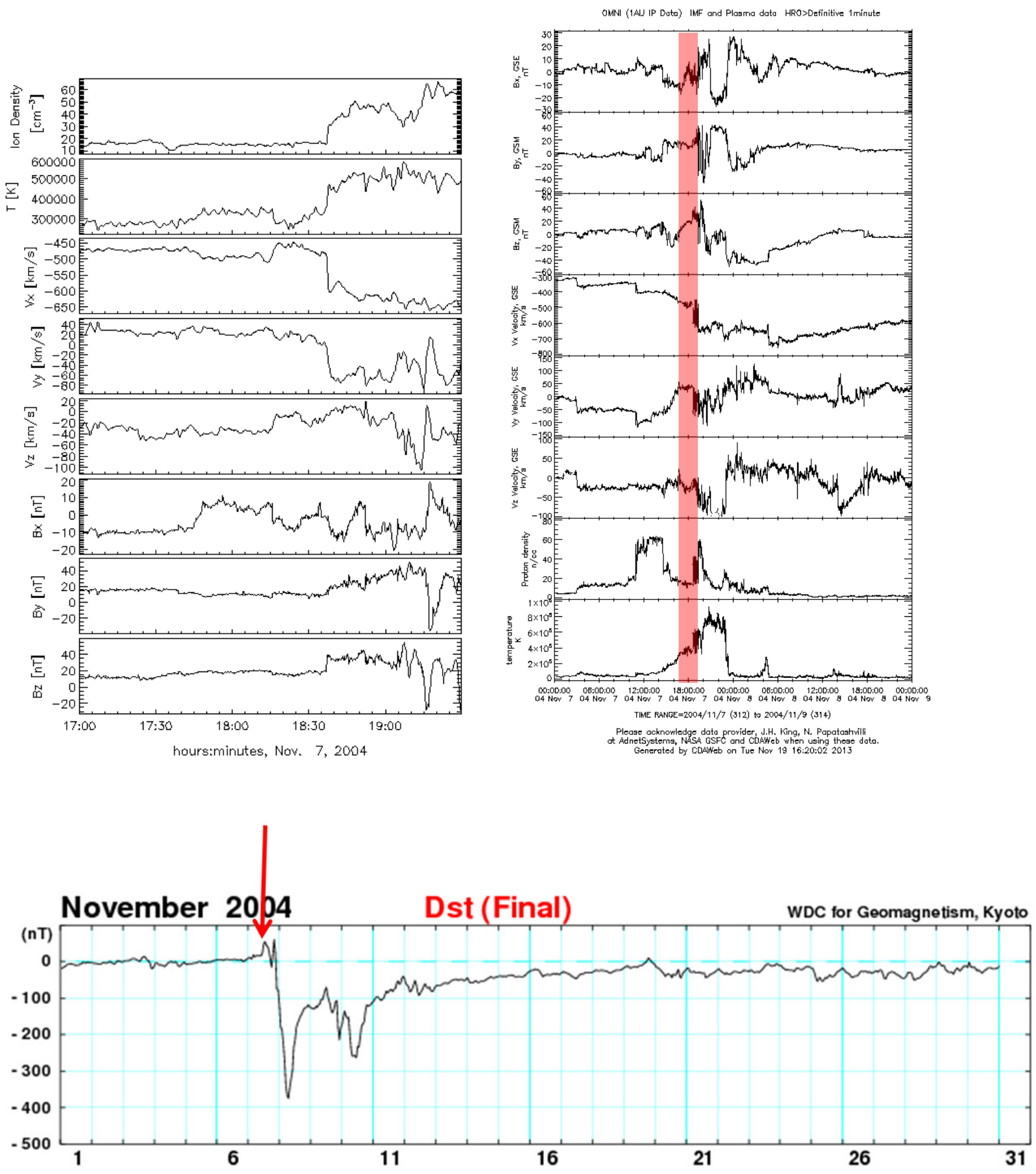

Figure 6-1: Top: Solar Wind Data from WIND, Left: over the course of the whole geomagnetic storm event. Right: throughout the onset phase. Bottom: Dst index for the storm event. [WDC for Geomagnetism, Kyoto Dst index service] 


\subsection{Spatial and Spectral Properties of the MHD Field}

The OpenGGCM can provide any field component for both magnetic and electric fields, but we choose to only retrieve $\mathrm{E}_{\mathrm{L}}, \mathrm{E}_{\phi}$ and $\mathrm{B}_{\mathrm{z}}$ since these are the only possible contributors to electron scattering (see Eq. (2.1.14) and (2.1.15) ). In this section we give a detailed examination of the field properties.

The first set of graphs (figure 6-2 to 6-4) display the global power spectral density of both the magnetic and electric fields. For each spatial grid a Fourier transform is applied on the time series, in which the frequency range is divided into $\Delta \mathrm{f}=1 \mathrm{mHz}$ bins and the mean, absolute amplitude plotted for each of those. It should be noted that mapping the spectrum of the magnetic field can be challenging because of the cubic decrease in magnitude versus L-shell. We apply Fourier transforms on the azimuthal gradient of $\left|B_{z}\right|$ as well, allowing some features to be better distinguished; however, the information about field amplitudes is still not given through this method. Also the radial gradient is included as it is another important parameter in the drift velocity equation (2.1.15). The dashed, thick line represents the magnetic contour line where the Roederer L-shell is defined at $\mathrm{L}=6.6$ at noon, and the thinner contours mark 4, 5 and $6 \mathrm{R}_{\mathrm{E}}$ respectively.

The next set of plots (fig 6-5 and 6-6) are the Fourier transform of $E_{\phi}$ and $B_{z}$ at specific L-shells employed at all points in MLT. We focus mainly on these two components since $\mathrm{E}_{\mathrm{L}}$ has been proven inefficient in scattering outer belt electrons (see chapter 4.4) and does not appear to have the significant wave amplitudes needed to make a noticeable difference. 
We also examine the time series of the field components in figure 6-8. The values are extracted at L $=6.8$ over all MLT's - this will be the L-shell to be focused on in this chapter. An azimuthal gradient of the magnetic field is included as well in order to circumvent loss of detail due to the compressional component.
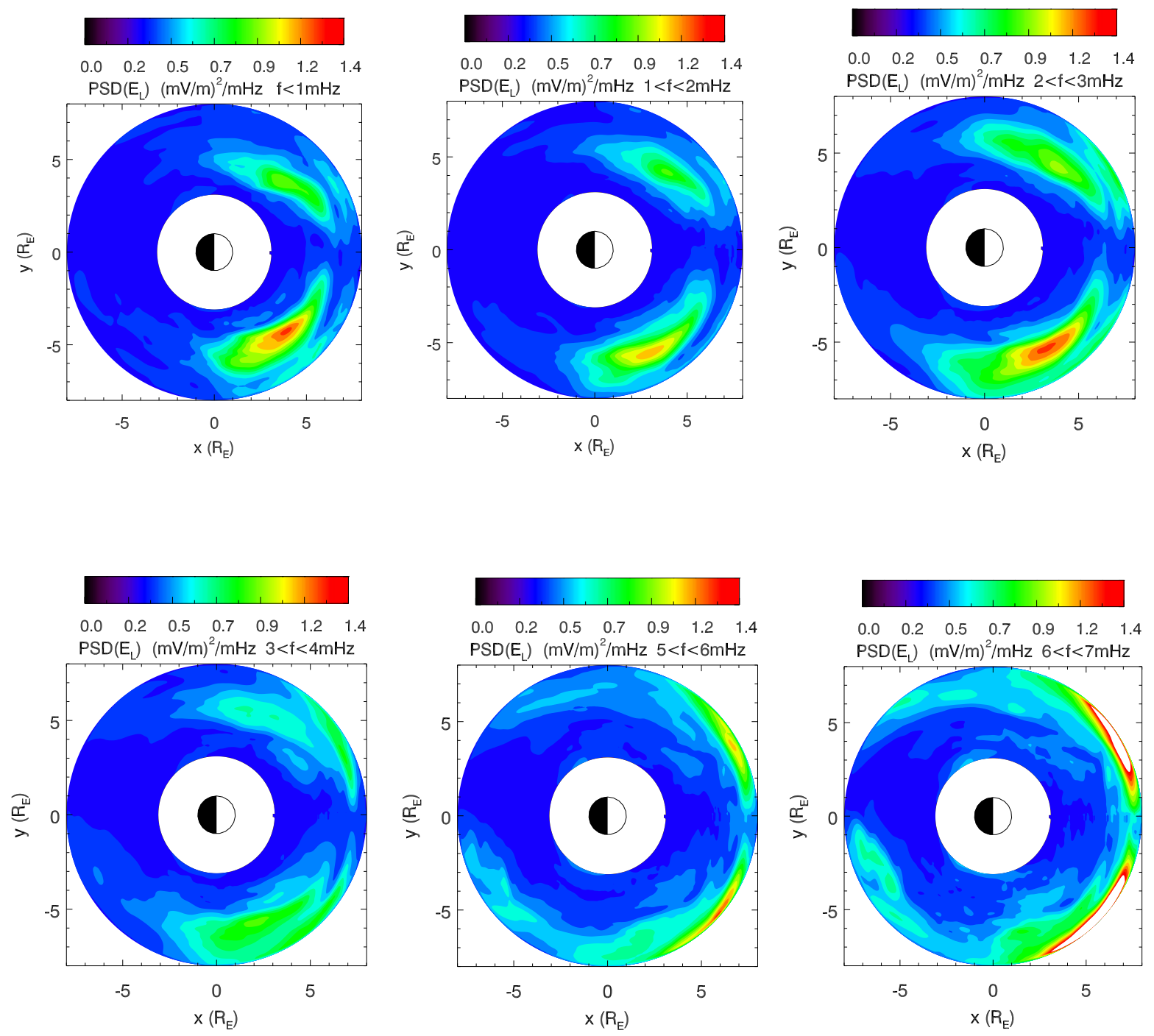

Figure 6-2: Global power spectral density maps of $E_{L}$ for frequencies 1-7 $\mathrm{mHz}$ and $\Delta f=1 \mathrm{mHz}$. 

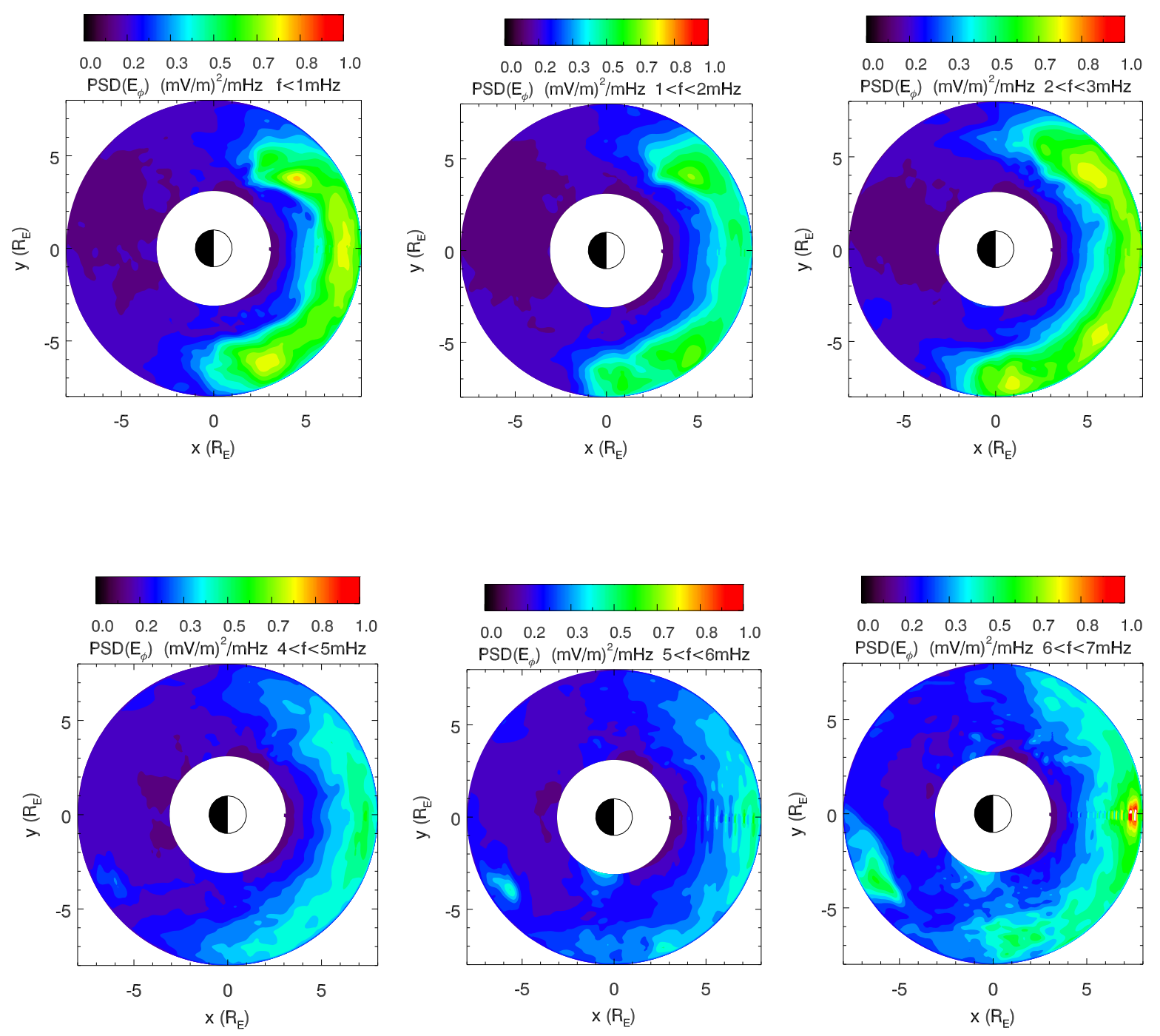

Figure 6-3: Global power spectral density maps of $E_{\phi}$ for frequencies 1-7 $\mathrm{mHz}$ and $\Delta f=1 \mathrm{mHz}$. 

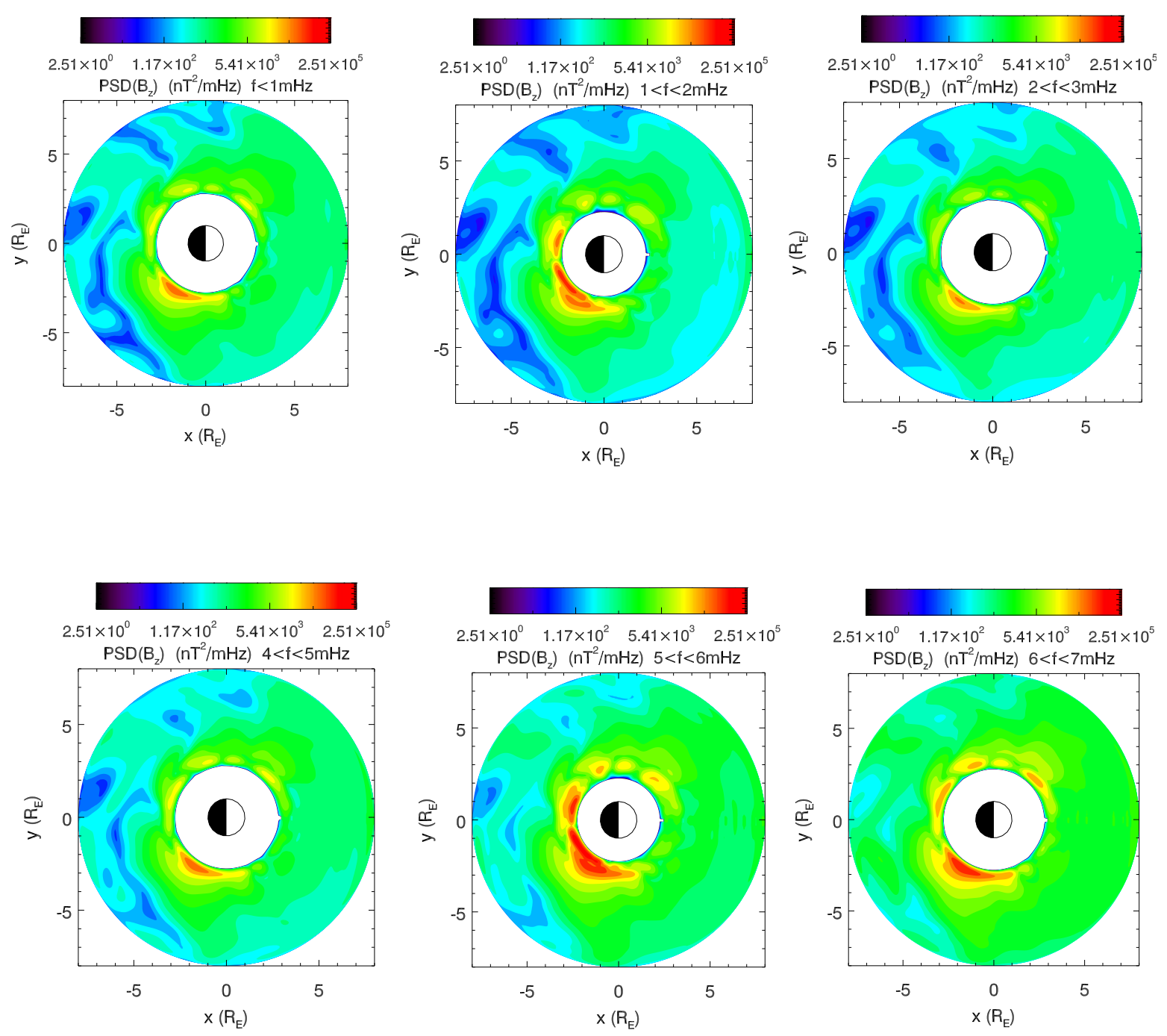

Figure 6-4: Global power spectral density maps of $B_{z}$ for frequencies 1-7 $\mathrm{mHz}$ and $\Delta f=1 \mathrm{mHz}$. 

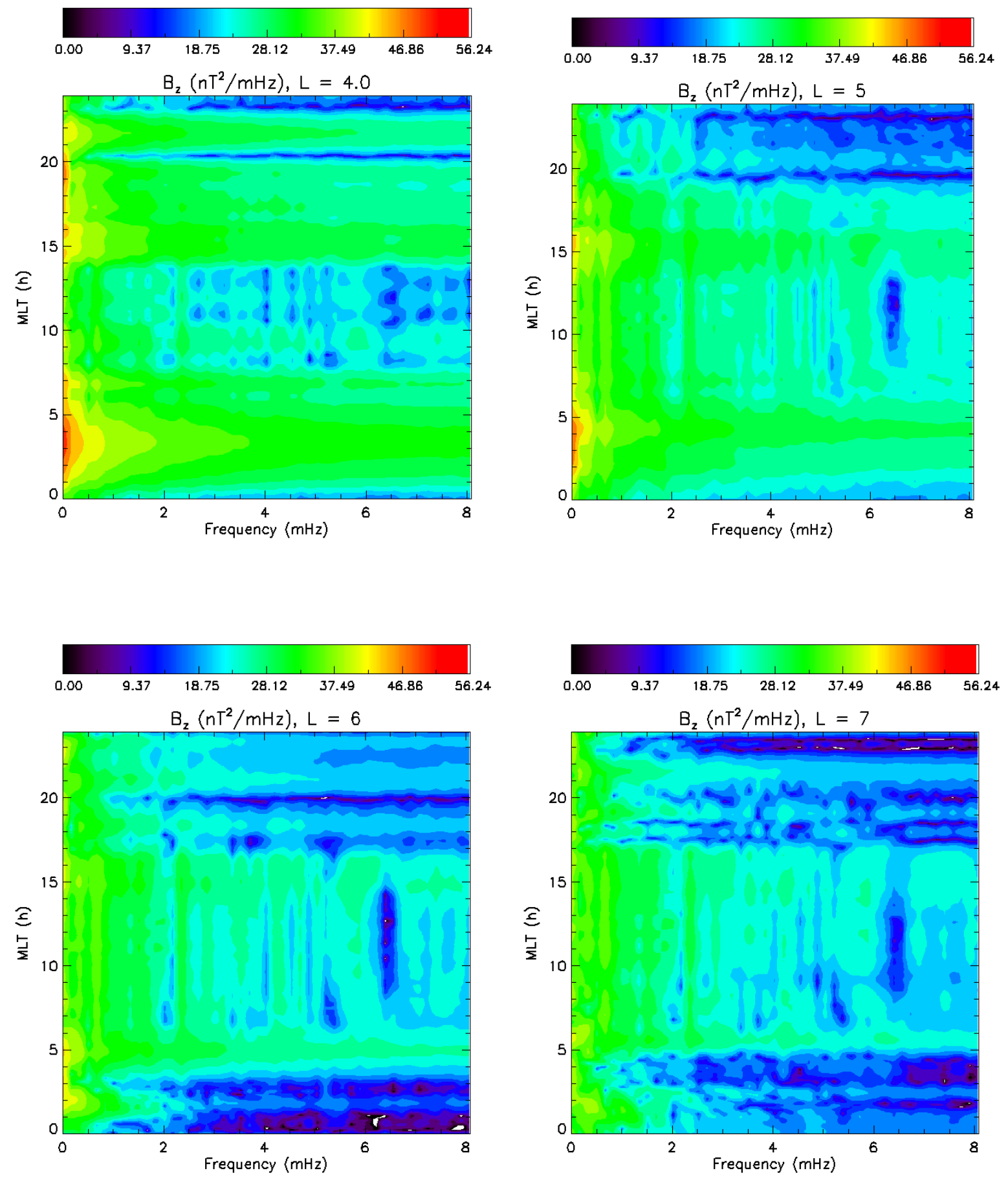

Figure 6-5: Spectrum of $B_{z}$ as a function of $M L T$ and $L$-shell $(L=4,5,6,7)$. 

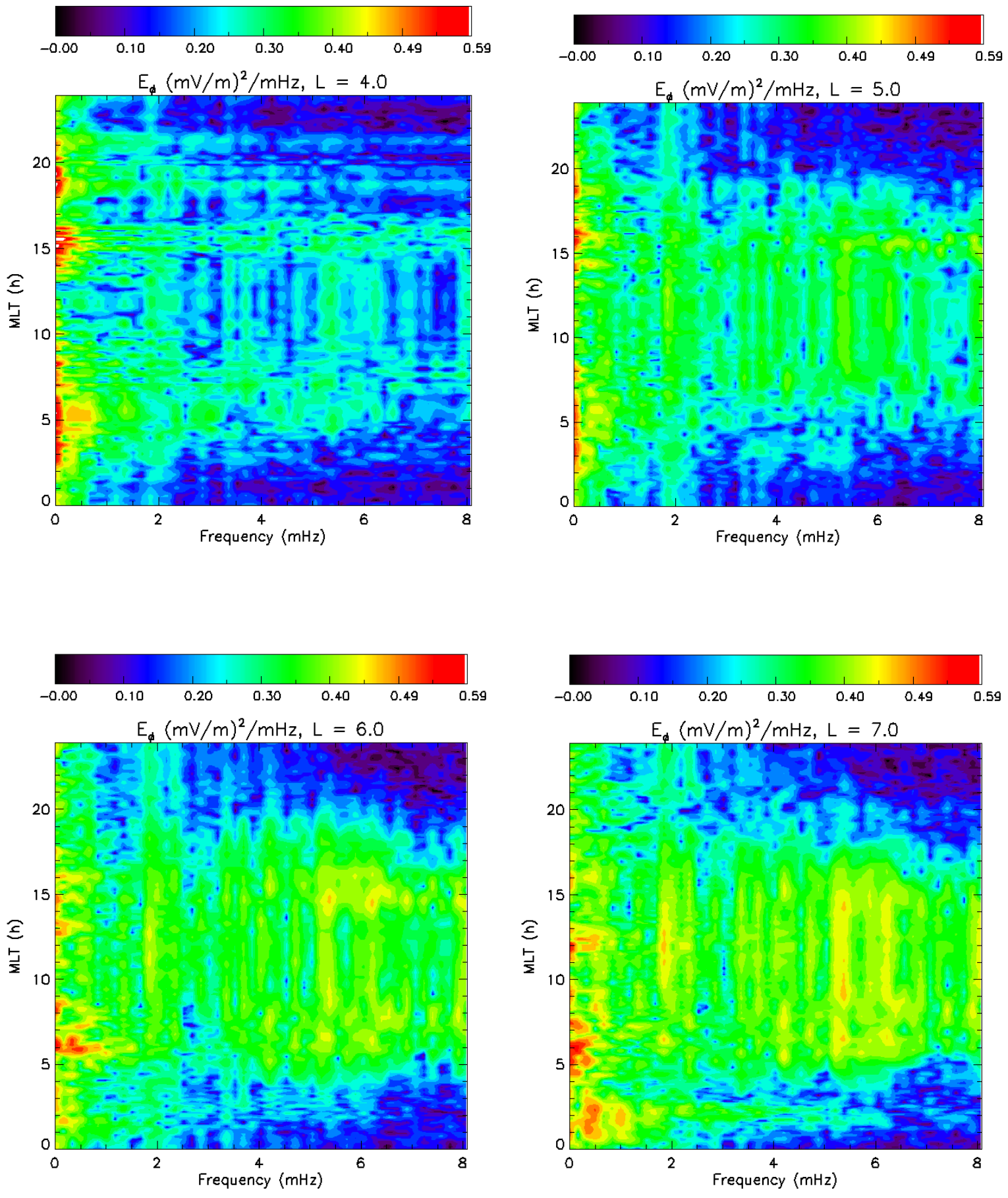

Figure 6-6: Spectrum of $E_{\phi}$ as a function of $M L T$ and $L$-shell $(L=4,5,6,7)$. 

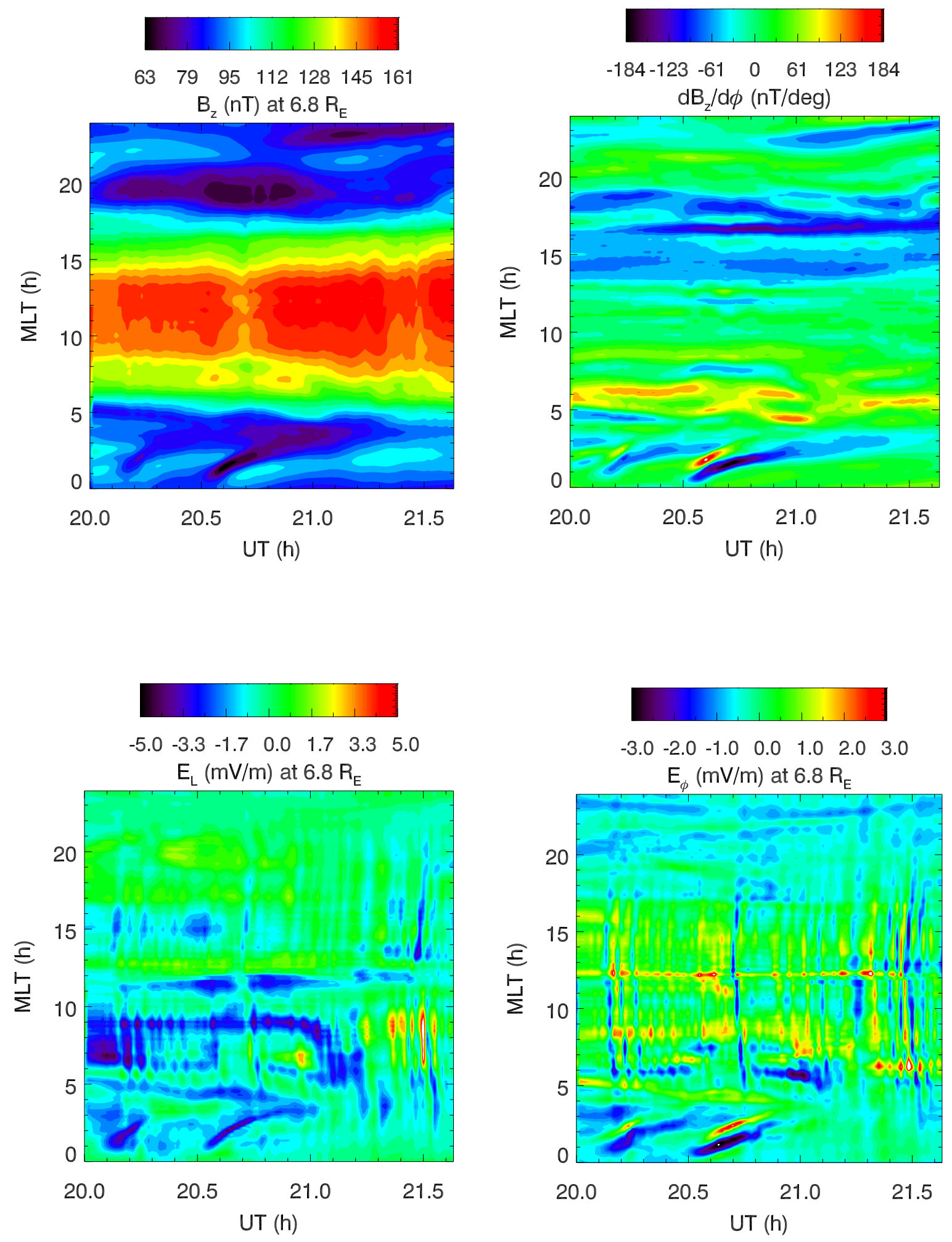

Figure 6-7: Time series of the fields at $L=6.8$, across all MLT's. 
It is apparent from figures 6-2 to 6-6 that ULF wave activity is most prominent at the dayside of the magnetosphere. In particular the $\mathrm{E}_{\varphi}$ component has distinct spatial boundaries at 16:00 and 06:00 MLT. It is reminiscent of the azimuthal sectors used in previous chapters and will be applied the same way in this chapter as well. There are irregular pulsations at about 20:0024:00 MLT that travel inward and counter clockwise. Since there are only two such pulsations and the fact that they travel relatively slowly against the drift path of relativistic electrons, it is assumed that their contribution to particle scattering is negligible. We will in later sections remove all field values outside the dayside sector except for the background magnetic dipole field.

Another feature visible in the time series graphs is an enhanced wave power at about 21:15 UT, when the storm onset is approaching. To maintain as simple a wave field as possible we will only consider the interval $\mathrm{UT}_{\text {int }}=20: 00-21: 15$ UT for wave-particle interaction evaluations.

The next set of graphs in figure 6-8 is constructed by finding the mean spectral value of each field component within this dayside sector at all L-shells, as well as the assigned time series. It is also useful to examine the wave field power within the dayside sector. A good indicator is from the absolute amplitudes of the wave fields, integrated over the time interval $\tau_{\text {int }}=1.15 \mathrm{~h}$ (figure 6-9). 

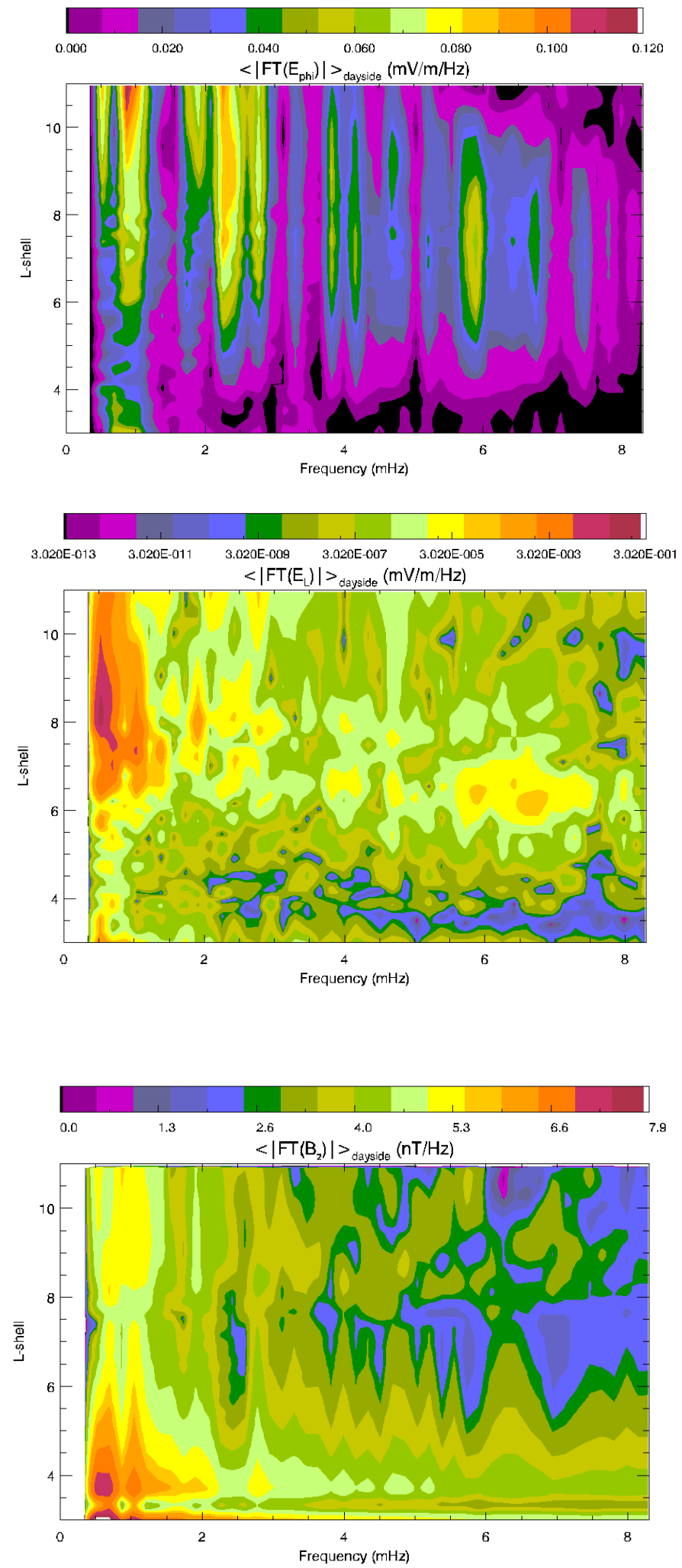

Figure 6-8: Mean spectra dayside field components: $B_{z}, E_{\phi}$ and $E_{L}$. 

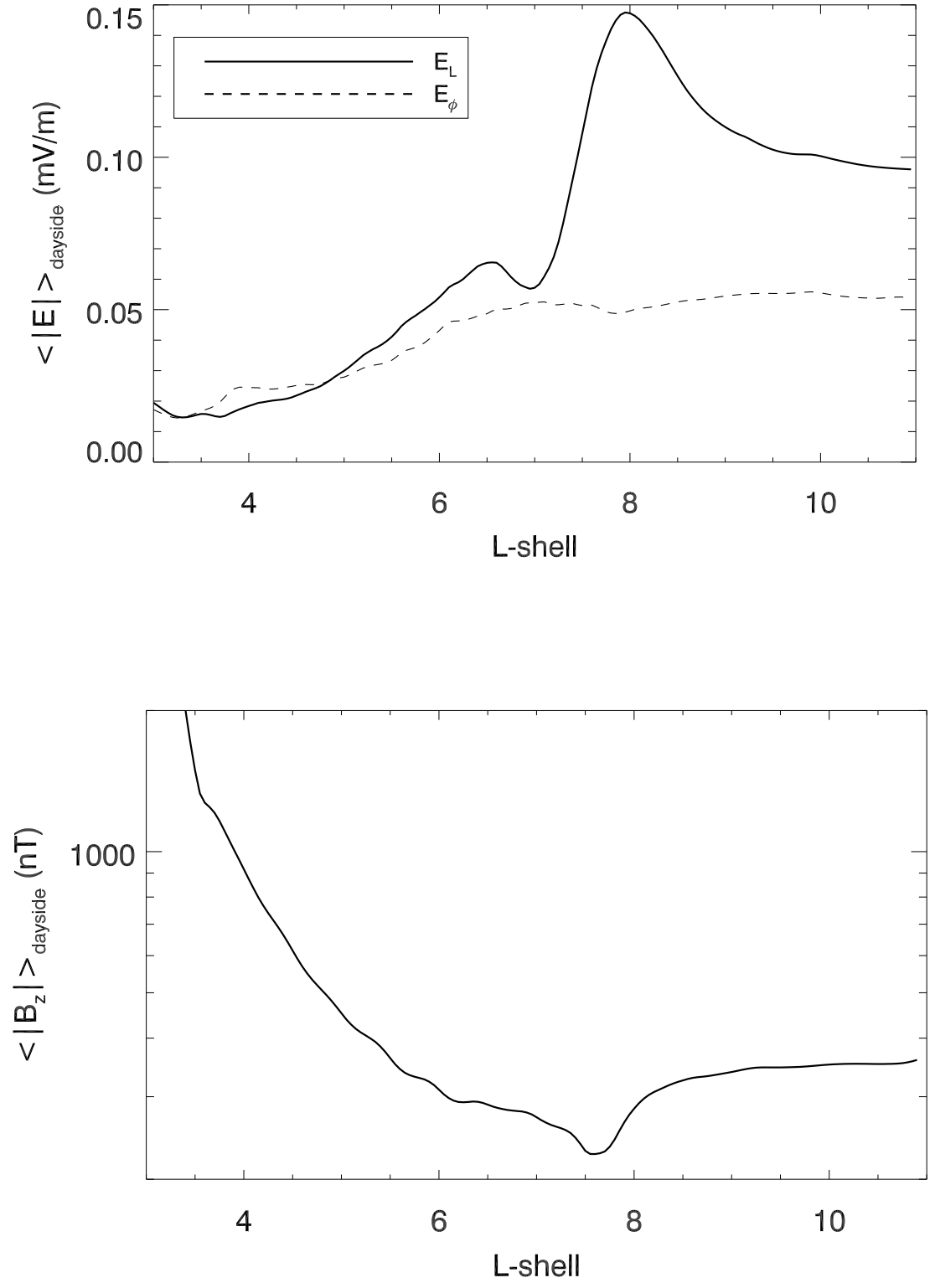

Figure 6-9: Mean total dayside (MLT: 16:00-06:00) field components: $B_{z}, E_{\phi}$ and $E_{L}$. 
With the help of this selection of graphs in our collection of figures (6-2 through 6-9) we can discuss basic properties of the ULF wave activity in this pre-storm interval:

The $\mathrm{E}_{\phi}$ field spectrum has a number of distinct monochromatic modes - in particular there is a global wave at $\mathrm{f} \sim 2.2-2.3 \mathrm{mHz}$ visible in figure $6-6$. Other low frequency modes exist mostly on the dayside at $\mathrm{f} \sim 0.5,0.9,1.8$ and $2.7 \mathrm{mHz}$. There are more modes at higher frequencies, which merge into a broadband range at $3.7<\mathrm{f}<6.9 \mathrm{mHz}$ that all are confined in the dayside sector.

The $E_{L}$ field does not cover the dayside sector within the magnetosphere like the $E_{\phi}$ and $B_{z}$ components. Instead, for $\mathrm{f}>4 \mathrm{mHz}$, this field component is more localized in MLT with two wedges appearing between dusk-noon and noon-dawn, where the former has the strongest amplitude. We speculate that since the spatial location is narrow in MLT, field-line resonances are producing these wave fields.

The spectrum of the $\mathrm{B}_{\mathrm{z}}$ component is dominated by the lowest frequencies with peaks at $\mathrm{f}=0.6$ and $1.1 \mathrm{mHz}$. The rest of the spectrum falls with increasing frequencies, i.e. obeys a power law with frequency and space dependent values. Like for the $\mathrm{E}_{\phi}$ spectrum, the wave activity is concentrated within the dayside sector, most prominently for $\mathrm{L}>5$. There is a radial wave structure centered at noon that becomes weaker with increasing frequency.

The location of the magnetopause is hinted by the strong $E_{\mathrm{L}}$ field components stretching along the dayside flanks at $\mathrm{L}>7.0$ for $\mathrm{f}<5 \mathrm{mHz}$ (see figure 6-2). The magnetic field spectrum and integrated power in figures 6-8 and 6-9 point out the magnetopause at $\mathrm{L} \sim 7.5-7.7$ at noon where a trough exists in the values. The discrete modes within the magnetosphere match with the frequencies in solar wind, thus a direct ULF wave transition across the magnetopause 
boundary takes place. The same is observed for the $\mathrm{E}_{\phi}$ field with distinct modes originating at $\mathrm{L}>9$ that penetrate deep into the magnetosphere.

An important parameter to be determined is the wavenumber which can be deduced from using spatial Fourier transforms on the wave fields. A time interval UT 20:32-20:42 is selected, where at $\mathrm{L}=6.8$ and for each time step, Fourier transforms are applied onto the dayside sector. Each time grid yields azimuthal wave vectors which are then averaged together for the entire UT interval, shown in figure 6-10. We do this procedure for specific frequency ranges $(0.3<\mathrm{f}<1.2 \mathrm{mHz}, 1.3<\mathrm{f}<3.2 \mathrm{mHz}, 3.3<\mathrm{f}<7.9 \mathrm{mHz})$ that were filtered through in the time series. This procedure gives a wavenumber with the end points set at the azimuthal boundaries at 16:00 and 06:00 MLT, which we can call $\mathrm{m}_{\mathrm{sec}}$. All field components are dominated by $\mathrm{m}_{\mathrm{sec}}=0$ or $\left|\mathrm{m}_{\mathrm{sec}}\right|=1$, at this L-shell. The $\mathrm{E}_{\phi}$ and $\mathrm{B}_{\mathrm{z}}$ modes appear strongest at $\mathrm{m}_{\mathrm{sec}}=0$, while the $\mathrm{E}_{\mathrm{L}}$ mode has a peak at $\left|\mathrm{m}_{\mathrm{sec}}\right|=1$. For all cases it appears that the wavenumbers are similar for frequencies between $1.3-7.9 \mathrm{mHz}$, while the lowest frequencies compose differently.

We used global wave numbers $\mathrm{m}$ in the previous chapters, and will continue to do so here. The sector wave number is simply obtained from $m=\left(24 / \Delta \phi_{\mathrm{sec}}\right) \mathrm{m}_{\mathrm{sec}}$. For the assumed sector width $\Delta \phi_{\mathrm{sec}}=14 \mathrm{~h}$ in this chapter the multiplier is a non-integer, leading to non-integer global wave numbers. For $\left|\mathrm{m}_{\mathrm{sec}}\right|=1$, the global value is $|\mathrm{m}|=24 / 14=1.7$. 

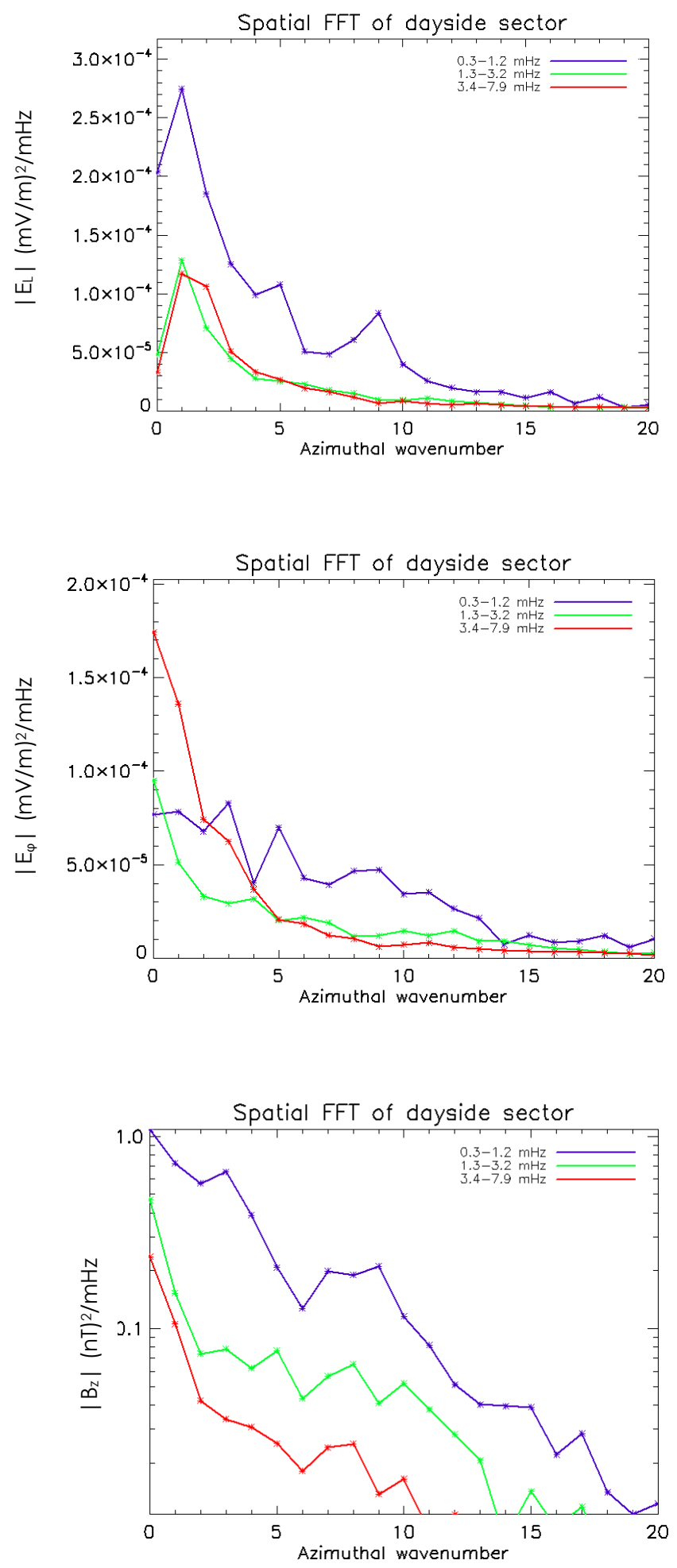

Figure 6-10: Wave numbers for dayside sector waves at $\mathrm{L}=6.8$. 
In conclusion, the MHD fields for this pre-storm event were found to be steady for over an hour, with different spectral profiles depending on the field component. It is a good case to build tests on for particle scattering as there are no overly complicated magnetospheric field dynamics involved. Furthermore, the fact that there is a dayside sector with distinct boundaries is a good complement to the discussions in chapter 5 . We will continue to explore diffusion of electrons in this chapter due to the relatively long time frame available from the OpenGGCM data.

\subsection{Testing the Particle Simulation}

To apply realistic MHD fields, such as the case discussed in this chapter, in the test particle simulation used throughout this thesis, one must take extra care. From a technical stand point the simulation becomes more prone to errors since the MHD fields have to be loaded into the guiding center code as a grid with polar coordinates, and each particle subjected to the grid values through interpolation in both space and time. In the previous chapters, the values were calculated locally for each time step and particle in a scheme which requires no interpolations,

thus provides better accuracy. The MHD frames have the time resolution of $\Delta \tau_{\text {grid }}=60 \mathrm{~s}$ and since the particle simulations run at a faster rate at $\Delta \tau_{\text {sim }}=1 \mathrm{~s}$, we apply a linear interpolation between these frames. The spatial resolution is $\Delta \mathrm{L}=0.1$ and $\Delta \phi=1^{\circ}$ ( $4 \mathrm{~min}$ in MLT). The original resolution given in Cartesian coordinates for the original data files is $\Delta x=\Delta y=0.1 R_{E}$, thus any better cannot be achieved in $\Delta L$. 
A test simulation is performed before taking on the MHD field. A magnetic field of the function form

$$
\begin{gathered}
B=\frac{B_{0}}{L^{3}}+B_{c} \cos \phi+B_{l o c} \\
B_{c}=\frac{B_{0}}{L^{3}}(0.05+0.06 L)(1-0.1 \cos (0.01 t)) \\
B_{l o c}=30[1+\cos (6 \phi-\pi)], 1.05 \mathrm{rad}<\phi<2.09 \mathrm{rad}
\end{gathered}
$$

is applied on the same grid coordinates as the MHD field is assigned onto. Its components are a core magnetic field dipole, a compression term which oscillates in amplitude with time, and a local, spatial perturbation ranging between $60^{\circ}<\phi<120^{\circ}$. It brings up a similar spatial dependence as one can expect in extreme cases in the MHD fields; a small range perturbation in azimuth, but large in amplitude.

Applying this magnetic field onto a grid with $\Delta \mathrm{t}_{\text {grid }}=60 \mathrm{~s}$ and running it with $\Delta \mathrm{t}=3 \mathrm{~s}$ using the interpolation approach for both spatial and temporal coordinates, provides the result given in figure 6-11. We compare the simulation using interpolations to another simulation run with ideal fields directly calculated in the particle frames. In total there are electrons distributed in $\mathrm{L}_{0}=4.0-5.0$, with $\Delta \mathrm{L}=0.1$, and initial MLT range 00:00 to 24:00 with 2 hour separation.

Overall the energy trends between the simulations look similar with differences that range from minor to major. To better distinguish the accuracy of the interpolation method, we compare the electron L-shell position found in the interpolation simulation with the reference simulation of direct calculations, which gives the error function: 


$$
\text { Error }=\frac{L_{d}-L_{i}}{L_{d}} \times(100 \%)
$$

Each electron experiences a maximal scattering $\Delta \mathrm{L}_{\max }$ at specific points in time, for which we sort them in ascending order (figure 6-12a) and show the error. Figures 6-12b and 6-12c pick out two electrons with one launched at $\mathrm{L}_{0}=4$, with an extreme at $\Delta \mathrm{L}_{\max }=0.6$, and another launched at $\mathrm{L}_{0}=5.0$ and $\Delta \mathrm{L}_{\max }=1.0$. There are spikes in inaccuracy for both picked out electrons associated with the passing of the locally perturbed magnetic field. For clearer comparison, intermediate averaged values are found through a smoothing window spanning the full drift orbit period for each electron, plotted in red in the same figures. The average Lshell displacement error for the electron with $\Delta \mathrm{L}_{\max }=0.6$ is $<5 \%$ within 1.5 hours of simulation time, while the error for $\Delta \mathrm{L}_{\max }=1.0$ is $<10 \%$ for the same time range. The error increases dramatically for the few electrons that scatter beyond $\Delta \mathrm{L}_{\max }>1.5$.

With this test simulation experiment it can be considered feasible to run test particles in MHD fields similar to or the same as the one selected for this case study. The wave fields are not as strong as in the test field, and the electrons will generally not scatter significantly beyond the typical values found here.

Figure 6-11 (next page): Individual electron trajectories from simulations, where (left) direct calculation of the magnetic field in the particle frame were conducted, or (right) a grid of the magnetic field was used and interpolated onto the particle frame. All plotted electrons were launched at 00:00 MLT, $W_{0}=3.0 \mathrm{MeV}$ and $L_{0}=4.0-5.0$.

Top: Perpendicular energy versus time. Middle: L-shell position versus time. Bottom: Lshell position versus $M L T$ 

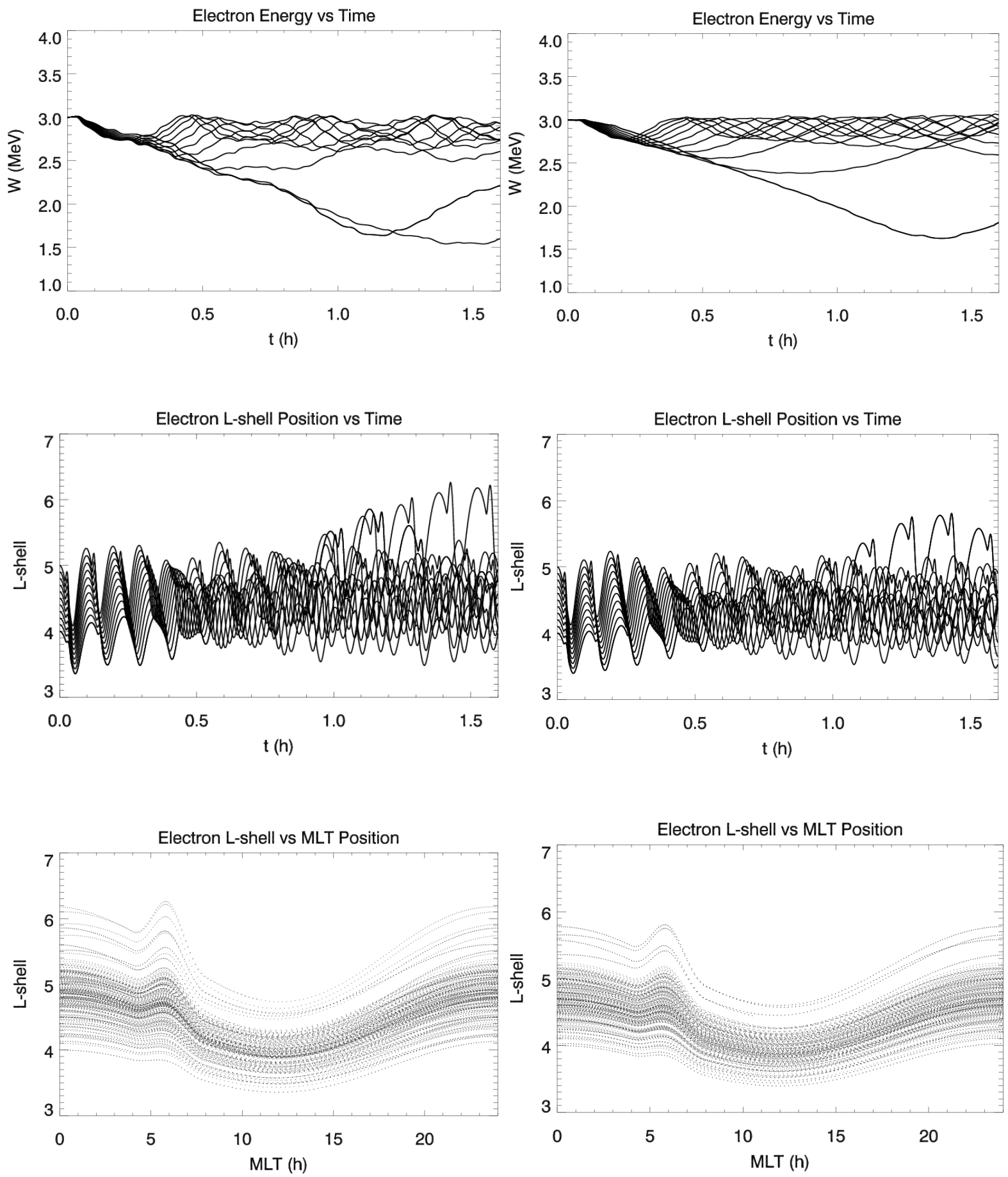

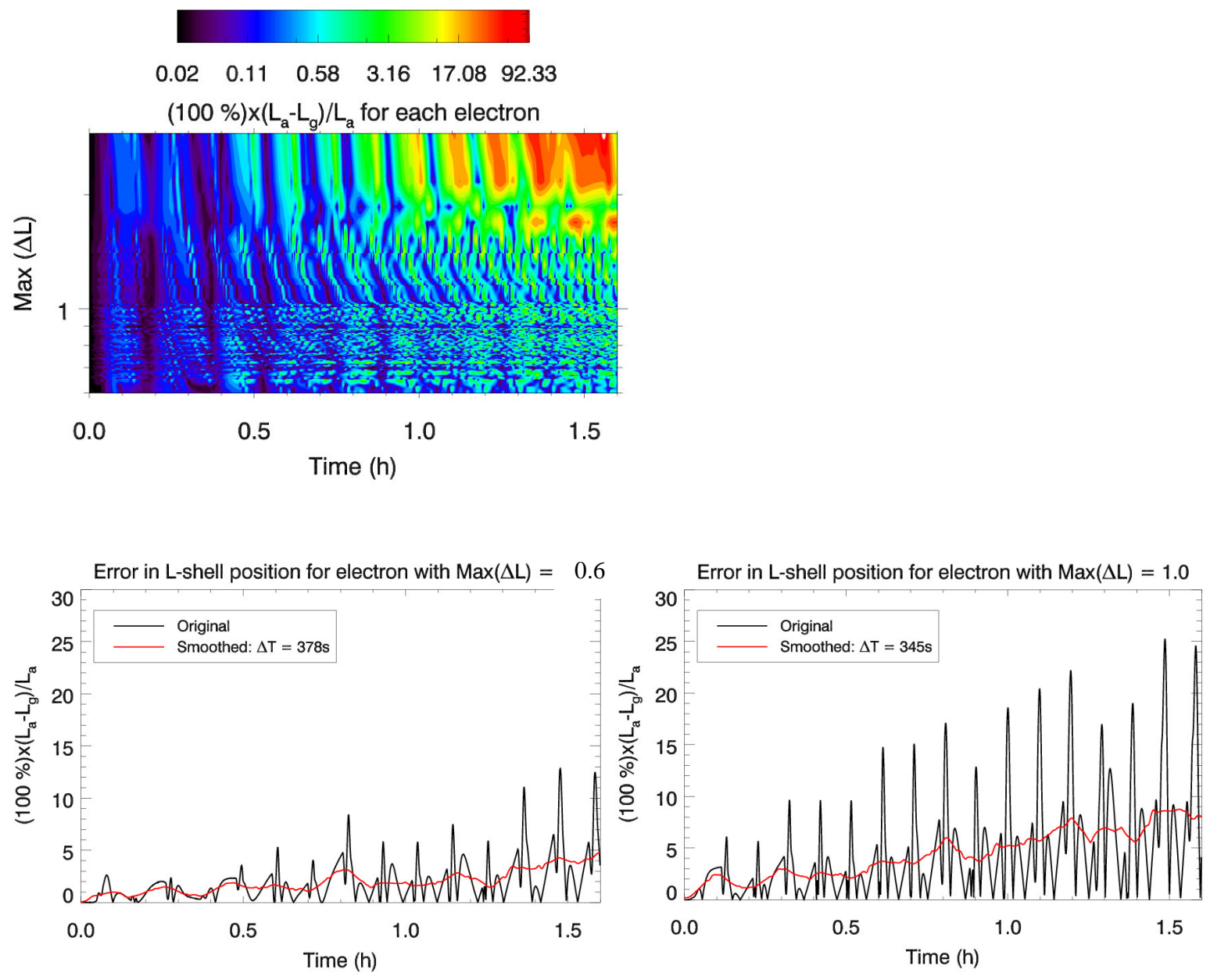

Figure 6-12: Error in L-shell position throughout the simulation. Top: Due to max(dL),

Bottom: At $\Delta \mathrm{L}_{\max }=0.6$ and $\Delta \mathrm{L}_{\max }=1.0$ 


\subsection{Modeling and Simulating Electron Radial Diffusion}

The main objective of this section is to evaluate the diffusion rate for electrons populated in the pre-storm event described in sections 6.3-6.4. We know from chapter 4 that the $E_{\phi}$ component dominates wave-particle scattering and is thus expected to be the main driver here as well. All wave fields will be confined in the same dayside sector as discussed in section 6.4 and electrons will be tested with both test particle simulations and variance calculations.

One modeling complication is electron scattering due to the radial distribution of wave field power for the electrostatic and magnetostatic components. This has not been considered in earlier chapters of this dissertation and must be simplified in this case study as well. Fortunately the radial gradients are weak for $\mathrm{E}_{\phi}$ at $6.0<\mathrm{L}<7.5$, and relatively weak for $\mathrm{E}_{\mathrm{L}}$ and $\mathrm{B}_{\mathrm{z}}$ for these L-shells as well (figure 6-9). The procedure is to extract the wave field at an L-shell and apply the same on all other L-shells. Here it is chosen to be extracted at $\mathrm{L}=6.0-$ the same which will be the launching position for the electrons. If electrons are launched at lower L-shells they would encounter weaker wave fields and not scatter as much. At higher L-shells the electrons instead risk crossing the magnetopause boundary, making such selection less desirable.

The spectrum of the electric field is best shown as an average over the dayside MLT range of 16:00-06:00 at $\mathrm{L}=6.0$ (figure 6-13). The lowest cutoff frequency is set to $\mathrm{f}_{\mathrm{c}}=0.34 \mathrm{mHz}$ in the spectrum, so that the longest time scale of fluctuations within the event coincides with $1 / f_{c}$, thus any convectional dynamics causing uncertainties in our wave-particle examination

will be eliminated. While the DC components for the wave electric fields are neglected in this 
approximation, the magnetic field still retains static values in the form of an ideal background dipole field. For the test particle simulations, the electromagnetic wave field is azimuthally smoothed with a window size of 6 degrees in order to improve the interpolation accuracy. A guide to this particular smoothing width is that the fastest drifting electrons at $f_{d} \sim 7 \mathrm{mHz}$, can move across three MLT grids in one time step, and there should not be any sharp gradients within that time interval in the path of the particle.
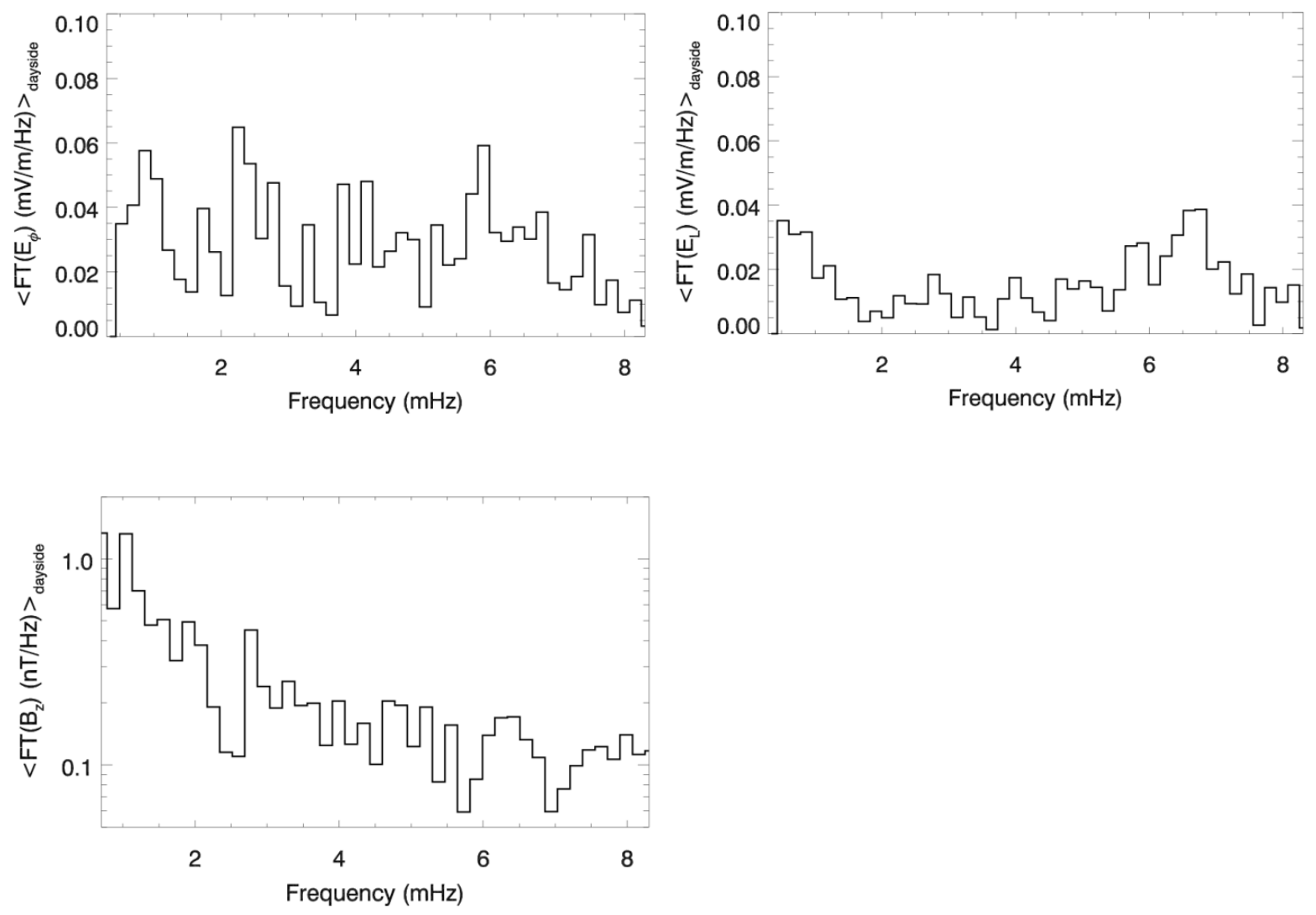

Figure 6-13: $E_{L}, E_{\phi}$ and $B_{z}$ spectra averaged over the MLT dayside sector 16:00-06:00 at $L=6.0$. 
In order to have a valid diffusive particle scattering there must be sufficient stochasticity in the system. It is possible to approximately calculate the value of K ( Eq. (2.2.14) ) from observing the spectral information in figure 6.13 and an example is given for the wave electric fields. Since the modes at $\mathrm{f}_{\mathrm{k}}<3 \mathrm{mHz}$ are defined within narrowband frequencies, we make an approximate calculation for electrons starting with a drift frequency of $f_{d}=6.33 \mathrm{mHz}$ which is well within the higher frequency broadband portion of the spectrum. Using an average spectral amplitude $\left|\mathrm{E}_{\phi}\right|=0.031 \mathrm{mV} / \mathrm{m}$ at $6.33 \mathrm{mHz}$, equation (2.2.10) gives $\Delta \mathrm{L}=0.16$, which translates to $\Delta \mathrm{f}_{\mathrm{d}}=0.086 \mathrm{mHz}$. The spectral resolution is $\Delta \mathrm{f}_{\mathrm{k}}=0.17 \mathrm{mHz}$ due to the restriction of using a time series of 1.15 hours. This gives a stochasticity parameter of $\mathrm{K}=\left(\Delta \mathrm{f}_{\mathrm{d}} / \Delta \mathrm{f}\right)^{2}=$ 0.26 which is far below the threshold value of $\mathrm{K}=1.03 \pm 0.23$ found in chapter 4.3 , thus there cannot be any transport across resonances and no intrinsic stochasticity. Moreover, the resolution of the frequency gives, from Eq. (2.3.1), a decorrelation time scale of $\tau_{\Delta f}=5760 s=1.6 h$ which exceeds the duration of the event itself, thus intrinsic stochasticity is again not a feasible driver for diffusion. As pointed out in the wave field analysis in chapter 6.2 there appears to be a temporary disruption in the oscillations at about 20:40 UT that may count as an extrinsic influence on randomizing particle transport, however only one such instance does not suffice in producing a diffusive response. Therefore the test particle simulations will be extended in time by a factor of 10 , where the field time series will be repeated as many times effectively connecting the last time step with the first in the next interval. Each repetition counts as a random jump in the particle scattering since position and phase of wave fields decorrelation. The total time span determines the uncertainty in the diffusion coefficient. 
Twelve hours is a long time interval for particle scattering due to wave fields, and as it turns out a significant portion of the electron population crosses over the magnetopause boundary at $\mathrm{L}>7.6$ at noon within this time span if $L_{0}^{*}=6.0$.

The magnetic dipole field of the MHD solution is asymmetric with respect to the day and night side, and this asymmetry must be taken into account for the particle simulations. For the purpose of maintaining a reasonable level of accuracy in the test particle simulations we approximate an ideal dipole field from the near-Earth field of the MHD model. Using a compression function of the same form as in equation (4.2.2), that is: $B_{c} \cos \left(\phi+\varphi_{c}\right)$, where $\varphi_{c}$ is the offset from noon, a best fit of the azimuthal profile of the magnetic field at $\mathrm{L}=6.0$ is conducted for each time grid throughout the interval (see figure 6-14). The parameters are $B_{c}=39.5 \pm 2.7 n T$ and $\varphi_{c}=-1.0 \pm 0.1 h$, of which the mean values are used in the particle simulations.
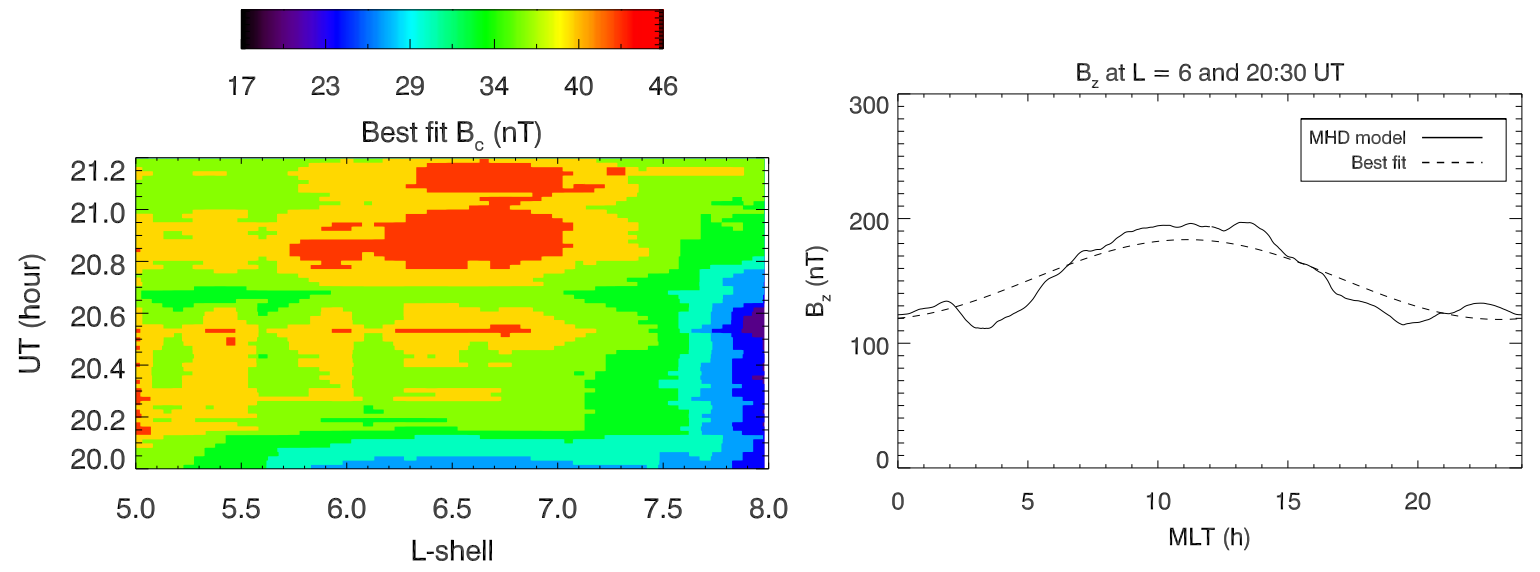

Figure 6-14: Left: Day-night asymmetric magnetic field compression strengths for interval $U T=20: 00-21: 12$, Right: Azimuthal best fit of the magnetic compression at one instance at $6.0 R_{E}$ 
The electron energies are set to range between $\mathrm{W}_{0}=0.5-7.0 \mathrm{MeV}$, with steps of $\Delta \mathrm{W}=0.1 \mathrm{MeV}$. In order to maintain uniform values of the adiabatic invariant $\mu$, we place the electrons along the B-magnitude lines instead of concentric rings. $\mathrm{L}^{*}$ is defined at the offset longitude $\varphi_{c}$ at $L^{*}=6.0$. The initial placements are spaced uniformly by the separation $\Delta \phi=\pi / 50$ or 14.4 min along the contour. At this Roederer L-shell the assigned particle energies have initial drift frequencies at $\mathrm{f}_{\mathrm{d} 0}=0.6-6.3 \mathrm{mHz}$.

When the magnetic magnitudes are asymmetric in MLT it is still convenient to use equation (2.3.5) to find the diffusion coefficient, but in terms of electron energy instead:

$$
D_{W W}=\frac{\left\langle(\Delta W)^{2}\right\rangle}{2 \tau}
$$

Conversion to $\mathrm{D}_{\mathrm{LL}}$ is straightforward through equation (2.2.12).

We start the investigation by simulating electrons with each field component separately, with all results shown in figure 6-15. The contribution to diffusion from the different field components is clearly separated by 1-2 orders of magnitude between each one, with $E_{\phi}$ being dominant and $\mathrm{E}_{\mathrm{L}}$ negligible. 


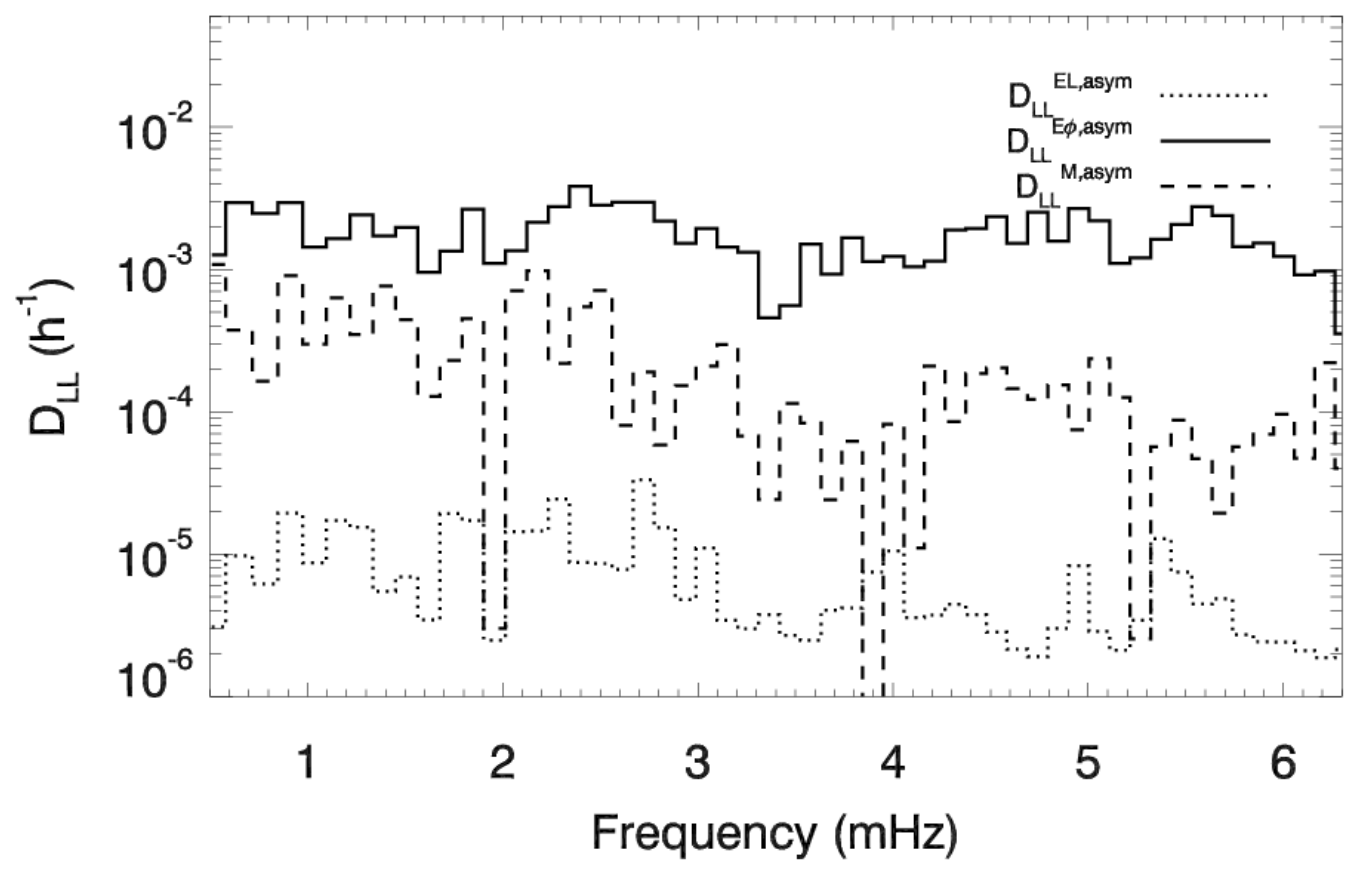

Figure 6-15: $D_{L L}$ for each MHD field component separately in an asymmetric dipole field.

Next we examine the effect of the $E_{\phi}$ and the $B_{z}$ components separately for two spatial configurations that gives in total 4 combined cases:

- A symmetric dipole field. An asymmetry provides additional terms in equations (2.1.14) and (2.1.15) compared to the symmetric field. Here it is tested how large the difference is between $\mathrm{B}_{\mathrm{c}}=0 \mathrm{nT}$ and $\mathrm{B}_{\mathrm{c}}=39 \mathrm{nT}$.

- Extraction of wave fields from the dayside sector at $\mathrm{L}=6.8$. Like for the extraction at $\mathrm{L}=6.0$, we apply this MLT field profile on all L-shells. From the field analysis in section 6.3 it is evident that the wave fields have nearly the same amplitudes and frequencies at both $\mathrm{L}=6.0$ and $\mathrm{L}=6.8$, mostly for $\mathrm{E}_{\phi}$, but also for $\mathrm{B}_{\mathrm{z}}$.

All 4 cases give similar $D_{L L}$ values for both $E_{\phi}$ and $B_{z}$ (figure 6-16). The magnetic dipole compression has no noticeable effect on diffusion for electrons launched at $L=6.0$. The 
similarity of the two wave fields evaluated at $\mathrm{L}=6.0$ and $\mathrm{L}=6.8$ cause similar diffusion pattern as well.
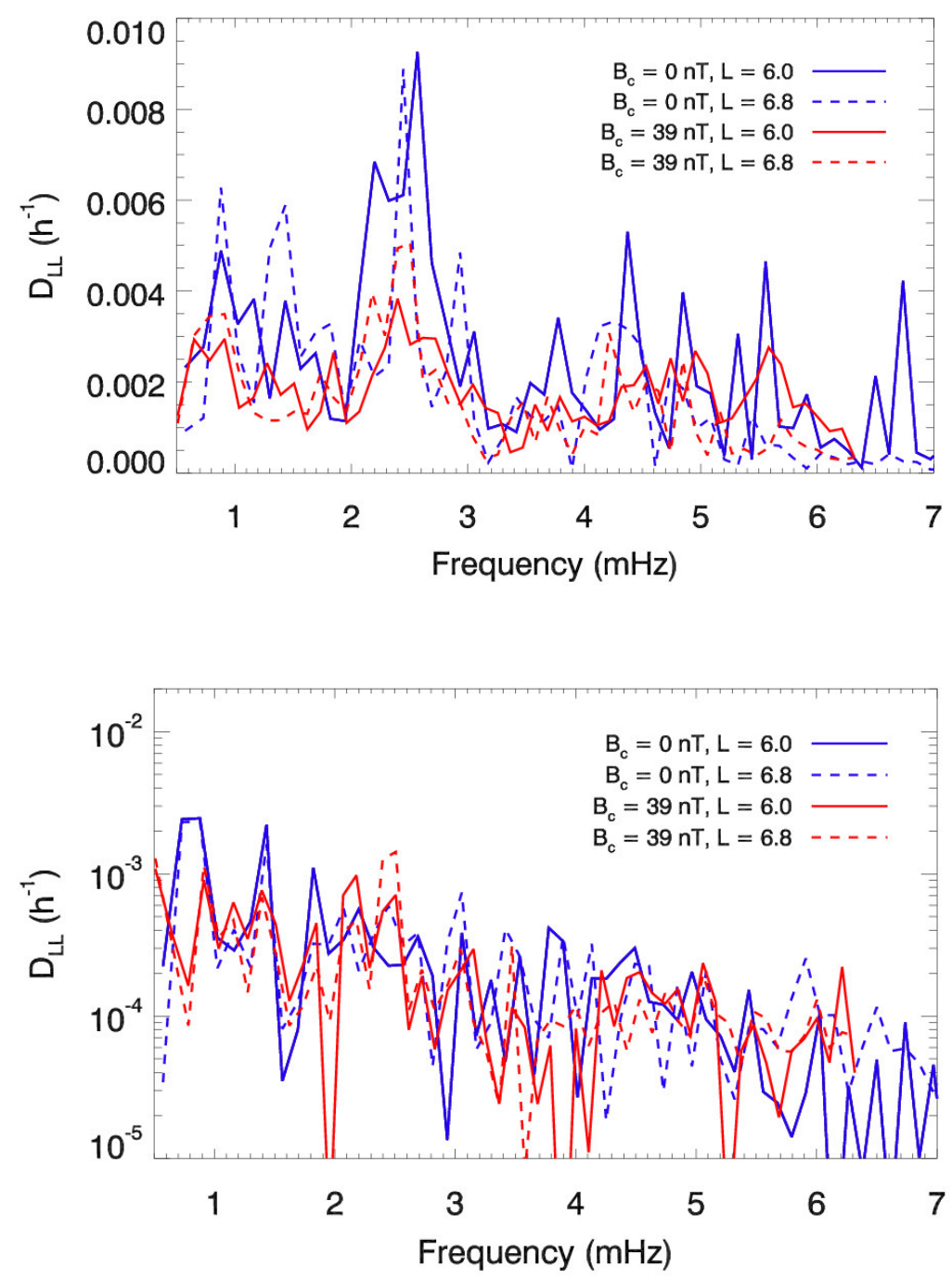

Figure 6-16: $D_{L L}$ for the 4 combined cases: $B c=0 n T, B c=39 n T, L=6.0, L=6.8$.

We use all 4 cases to create averaged diffusion values for each initial drift frequency to be used in further analysis here. Compared with the dayside averaged spectra (figure 6-17) there are a few things that can be concluded. For the electric field the narrowband peaks coincide reasonably well with the local power spectral density peaks at frequencies below $5 \mathrm{mHz}$, but 
not as well for higher frequencies. Since the low narrowband frequency modes match with the spectral amplitudes the global wave numbers must either be $\mathrm{m}=0$ or $|\mathrm{m}|=1$, which agrees well with the results in figure 6-10. Best linear fit for the high frequency, broadband range of $3.7<\mathrm{f}_{\mathrm{k}}<6.9 \mathrm{mHz}$ gives $\left|F\left(E_{\phi}\right)\right|^{2} \sim\left(5.9 \cdot 10^{-5} \pm 2.0 \cdot 10^{-4}\right) f_{k}$ and $D_{L L}^{E} \sim\left(-4.2 \cdot 10^{-4} \pm 1.1 \cdot 10^{-4}\right) f_{d 0}$. The spectrum can be considered overall flat $(\beta=0)$, while the $D_{\text {LL }}$ values decrease beyond $f_{d 0}=4.5 \mathrm{mHz}$, thus the local PSD alone cannot account for the diffusion rates.

Another thing to consider is various wave numbers, where for example the gaps in $\mathrm{D}_{\mathrm{LL}}$ at higher frequencies are due to the fact that those modes are constructed by $|\mathrm{m}| \geq 2$, leading to higher diffusion rates at lower drift frequencies. It is however possible that one must also consider the dynamic relation between the electron drifts, the electric field spectrum and the sector that it is contained within with $\tau_{\mathrm{r}}$ as the key parameter. 

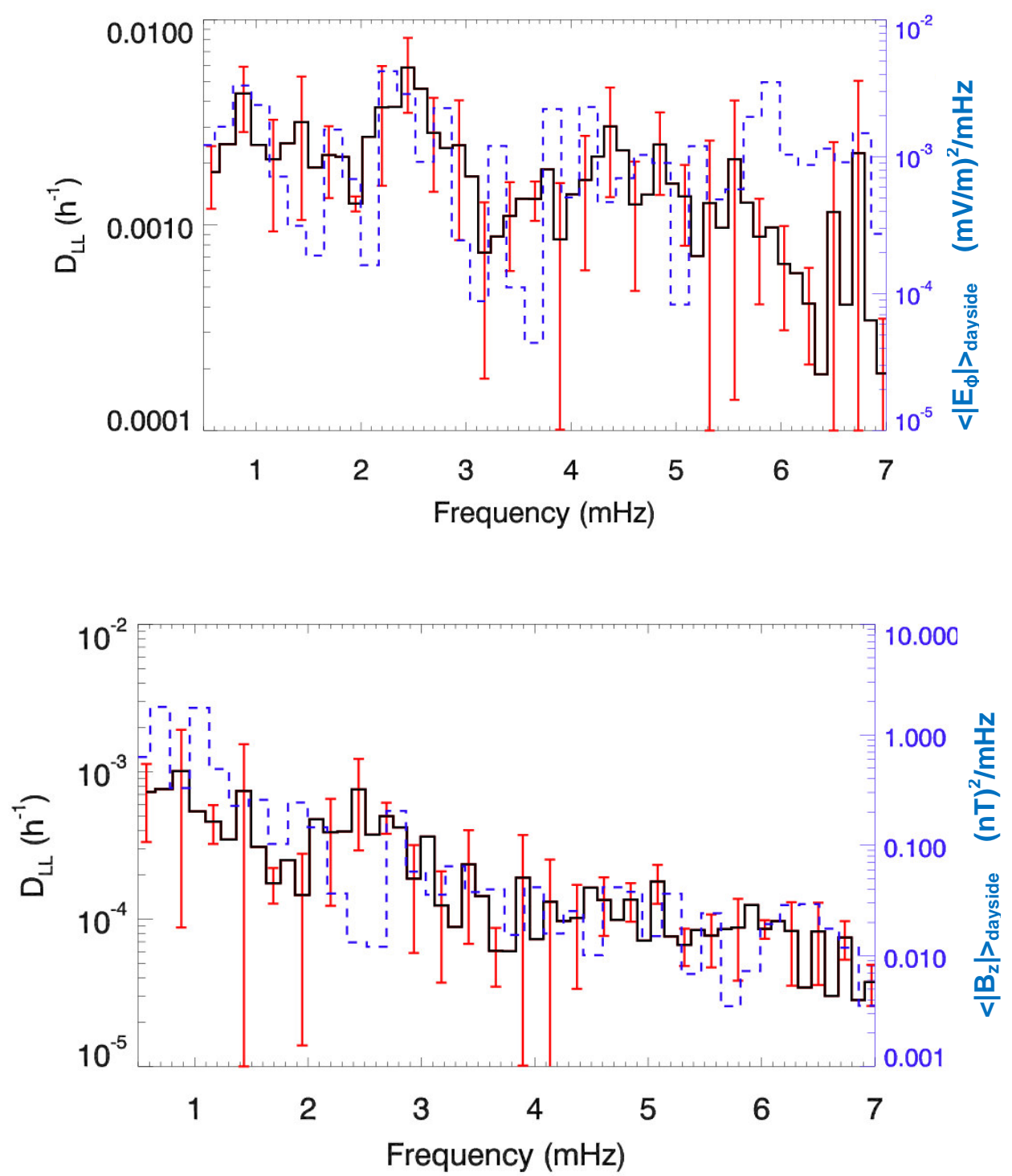

Figure 6-17: Averaged $D_{L L}$ for the 4 combined cases: $B c=0 n T, B c=39 n T, L=6.0$, $L=6.8$. The right axes are the dayside averaged spectra of the MHD fields at $L=6.0$. 
In contrast, the $\mathrm{D}_{\mathrm{LL}}$ frequency dependence on the magnetic field matches the inverse power slope of the spectrum - except at $2.5 \mathrm{mHz}$. We will leave this case and only treat the diffusion rates driven by wave electric fields in further discussions.

An addition to the particle simulations is the variance technique developed in chapter 5.3, which can further help understanding the drift frequency dependent $D_{L L}$ values. Local peaks cannot be evaluated with this method since each set of particles at one drift frequency gains contributions from all modes in the spectrum and are always considered to be within resonance. Thus for both the electric and magnetic fields, approximations are made for the wave fields used in the integrations. As the MLT-averaged Fourier spectral graphs for the electric field show in figure 6-13 there are at least three frequency ranges with distinct mode or modes. As approximations they are divided into intervals denoted "A", "B" and "C" with averaged amplitudes as follows:
A) $0.5-1.2 \mathrm{mHz}, \mathrm{E}_{0}=0.045 \mathrm{mV} / \mathrm{m} / \mathrm{Hz}$
B) $2.2-2.8 \mathrm{mHz}, \mathrm{E}_{0}=0.049 \mathrm{mV} / \mathrm{m} / \mathrm{Hz}$
C) $3.7-6.9 \mathrm{mHz}, \mathrm{E}_{0}=0.031 \mathrm{mV} / \mathrm{m} / \mathrm{Hz}$

These approximated amplitudes are plotted in figure 6-18. 


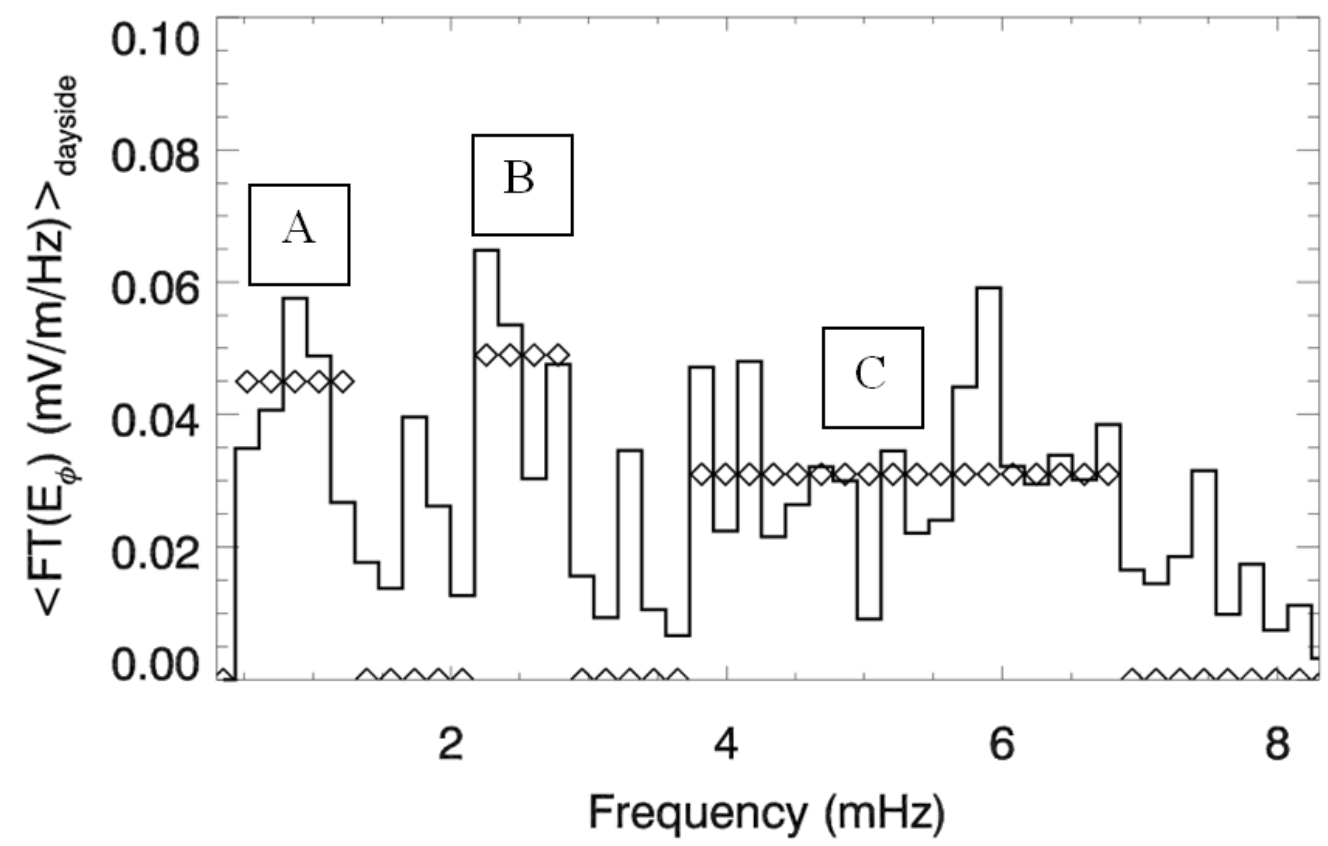

Figure 6-18: $E_{\phi}$ spectrum averaged over the dayside sector at $L=6.0$, with approximated amplitudes used for variance calculations.

Possible $\mathrm{D}_{\mathrm{LL}}$ values driven by the electric fields are calculated for different frequency ranges and wavenumbers. Figure 6-19 (top) display the results from interval $\mathrm{C}$ and the 3 lowest possible integer global wavenumbers. All modeled $\mathrm{D}_{\mathrm{LL}}$ is lower on average compared to the $\mathrm{D}_{\mathrm{LL}}$ from the particle simulation. The main reason is that all resonances are ignored, and only the averaged, integrated contribution from all modes within the interval counts. There can also be contributions from modes outside the $\mathrm{C}$ interval that are automatically left out in the variance calculations. Regardless, the graphs point at $|\mathrm{m}|=1$ being the most relevant contribution since it experiences a decreasing trend with $\mathrm{f}_{\mathrm{d} 0}$ similar to the simulation values.

Next we calculate the solution with all three intervals included simultaneously, plotted in figure 6-19 (bottom). The result is a peaked $\mathrm{D}_{\mathrm{LL}}$ value at $\mathrm{f}_{\mathrm{d} 0}=1.8 \mathrm{mHz}$ that decreases with increasing $\mathrm{f}_{\mathrm{d} 0}$. This is again compared to the simulated result, which is difficult to make any 
conclusions from by only looking at the unmodified data due to the local, resonant peaks. In order to extract the non-resonant contributions from all the modes within the selected intervals is to eliminate the resonant peaks from the data. One way is to re-simulate the electrons with each mode being phase reset at every drift orbit. This is however not feasible since each electron energy would have to be simulated separately with unique MHD solutions where each mode is reset at the drift frequency rate. A much faster and easier approach is to smooth, i.e. apply averaging of values, on $D_{L L}$ across frequency windows in the spectrum. It yields a similar effect on the $D_{L L}$ values as phase resets since the averages accounts for non-resonant modes as well. Here a smoothing window of $\Delta \mathrm{f}_{\mathrm{d} 0}= \pm 1.2 \mathrm{mHz}$ is applied on the simulated $\mathrm{D}_{\mathrm{LL}}$ due to the unmodified spectral electric field, which covers the gaps between the intervals A, B and $\mathrm{C}$. The distinct peaks disappear and the whole diffusion trend matches the analytical values better.

Finally, we simulate electrons in the MHD fields including more than one component in each case. For a symmetric background the $\mathrm{E}_{\phi}$ and $\mathrm{B}_{\mathrm{z}}$ components are applied, excluding $\mathrm{E}_{\mathrm{L}}$ since it has no effect on electron drift velocities when $B_{c}=0$ nT. From the results in figure 6-20 (top) it becomes evident that $D_{L L}^{E}+D_{L L}^{M} \neq D_{L L}^{E M}$, even so much as a factor of 2-3 times the additive value. 

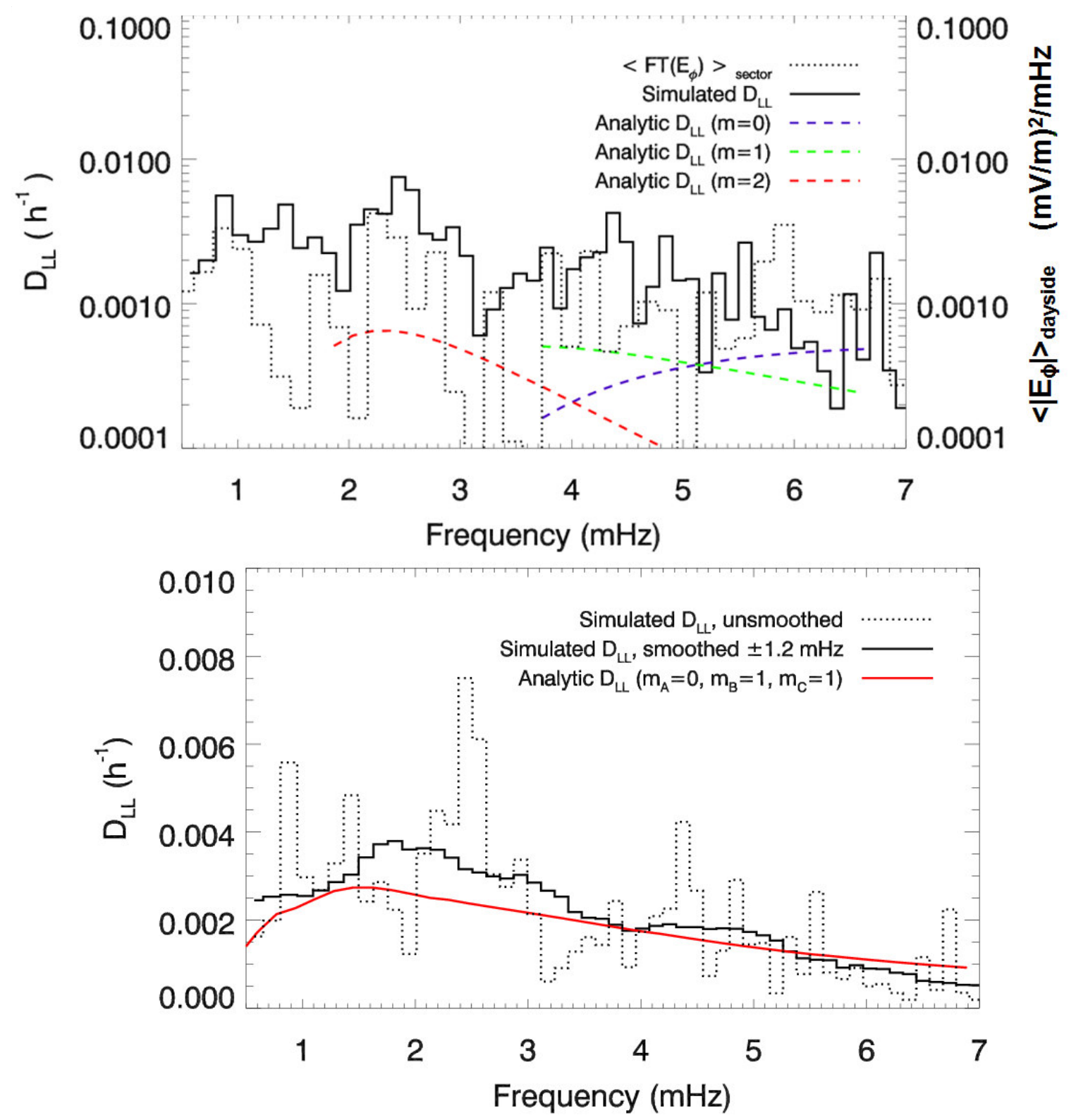

Figure 6-19: Top: $D_{L L}$ evaluated through simulations and the variance method (for different wavenumbers) applied for interval C, plus the power spectral density. Bottom: $D_{L L}$, evaluated through simulations, and smoothed to compare with the variance method for using all amplitudes intervals $A, B$ and $C$ together. 

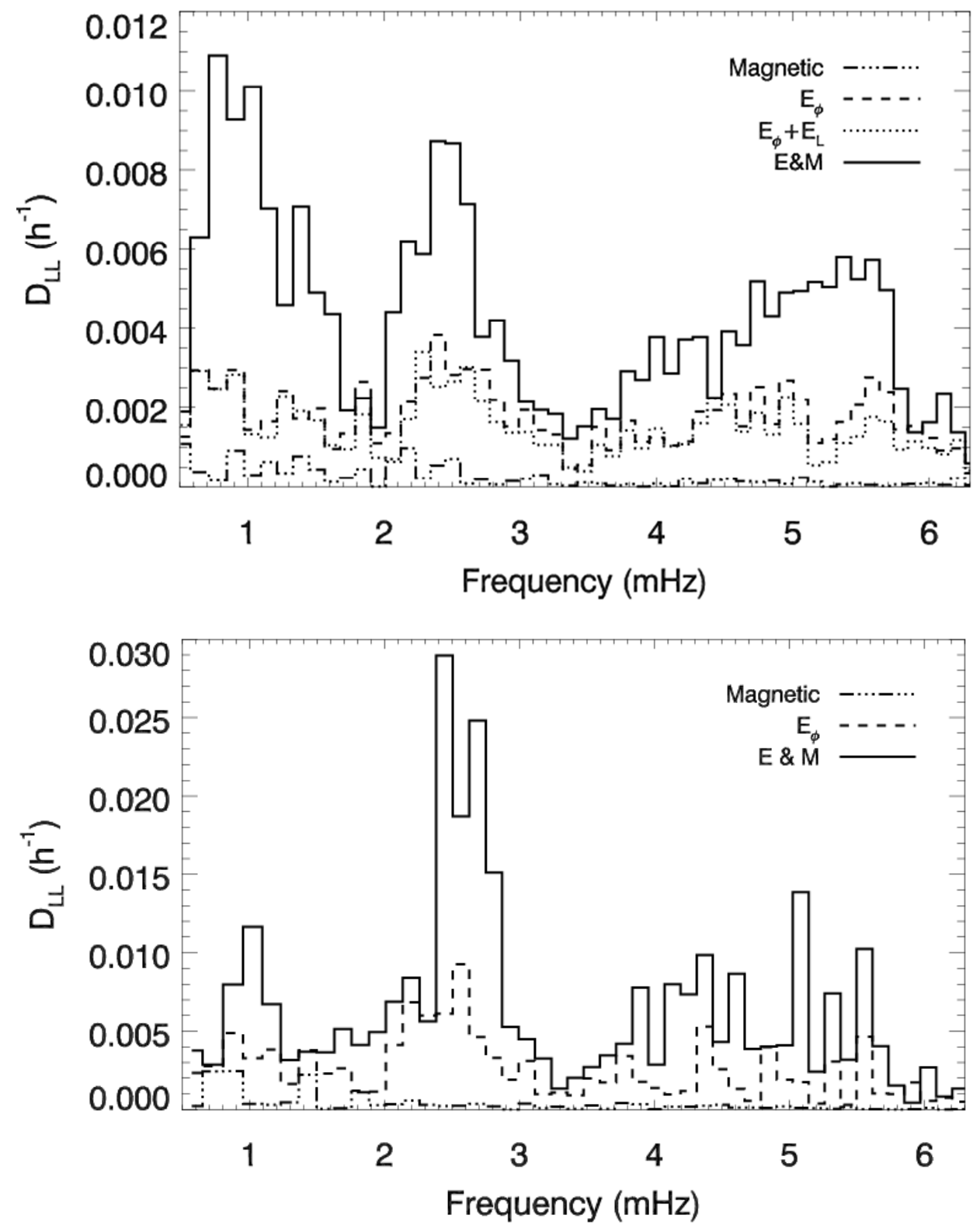

Figure 6-20: $D_{L L}$ for each wave field component separately, and combined. Top: Symmetric magnetic dipole $\left(B_{c}=0 \mathrm{nT}\right)$, Bottom: Asymmetric magnetic dipole $\left(B_{c}=39 \mathrm{nT}\right)$. 


\subsection{Conclusions}

We have investigated one pre-commencement interval at 17:00-18:12 on Nov 7, 2004 that exhibits ULF wave activity and evaluated the diffusion rate for electrons at the outer belt L-shells. The waves have distinctly different spectral and spatial features for each component in the electromagnetic field of the inner magnetospheric equatorial plane. Common for the three main field components capable of scattering electrons is that they mostly cover the dayside sector of the magnetosphere - especially $\mathrm{E}_{\phi}$. The electric wave field has an overall flat spectrum with local peaks, while the magnetic wave field drops inversely with frequency.

We have investigated the diffusive scattering of electrons. To enable diffusion there must be stochasticity in the wave-particle interactions which is possible by repeating the interval of the event 10 times in series while retaining the positions of the electrons for each repetition. Runs with each field component separately reveal large differences in $\mathrm{D}_{\mathrm{LL}}$, with $\mathrm{E}_{\phi}$ being dominant and $\mathrm{E}_{\mathrm{L}}$ negligible. We also compared results for both using $\mathrm{B}_{\mathrm{c}}=0 \mathrm{nT}$ and $\mathrm{B}_{\mathrm{c}}=39 \mathrm{nT}$ and conclude that there is no difference in $\mathrm{D}_{\mathrm{LL}}$ at any frequency for $\mathrm{E}_{\phi}$ and $\mathrm{B}_{\mathrm{Z}}$ components used separately. Neither of these two wave field components contributes to $\mathrm{v}_{\varphi}$ in $1^{\text {st }}$ or $2^{\text {nd }}$ order -

only the asymmetry in the magnetic dipole does. The $3^{\text {rd }}$ adiabatic invariant adjusts the exposure time for an electron passing across the dayside sector, but the difference in exposure time for $\mathrm{B}_{\mathrm{c}}=0 \mathrm{nT}$ and $\mathrm{B}_{\mathrm{c}}=39 \mathrm{nT}$ is not sufficient to make any significant difference in scattering widths in radial position and particle energy.

We then looked at the $\mathrm{E}_{\phi}$ case in detail with the additional aid from variance calculations. The dayside azimuthally averaged spectrum of the wave field has two main low frequency modes 
and an approximately flat broadband interval at higher frequencies. The simulated $\mathrm{D}_{\mathrm{LL}}$ matches well with the local PSD of the low frequency peaks, but the $\mathrm{D}_{\mathrm{LL}}$ model fails to account for the high frequency modes. The conclusion is that in addition to PSD as a diffusion driver at local frequencies, contributions from multiple modes exposing electrons to partial oscillations are a non-negligible factor. Variance calculations for the high frequency modes provide a clue that this is the case and that the modes have small wave numbers, where $|\mathrm{m}| \leq 1$ is dominant. We smooth the $\mathrm{D}_{\mathrm{LL}}$ profile across a frequency range so that resonant scattering is erased from the data, and compare with the variance calculations for the selected modes in the spectrum. The two $D_{\text {LL }}$ profiles match each other well which strengthens the hypothesis that non-resonant modes can give significant contribution to electron diffusion.

A third factor that we also demonstrated is the combination of wave field components into a single case, i.e. self-consistent fields. Adding $\mathrm{E}_{\mathrm{L}}$ alone to $\mathrm{E}_{\phi}$ in an asymmetric dipole field makes no difference, but including $\mathrm{B}_{\mathrm{z}}$ causes a discrepancy where $D_{L L}^{E M}=2$ to $3 \times\left(D_{L L}^{E}+D_{L L}^{M}\right)$. In this case the result is independent of $B_{c}$. 


\section{Summary and Discussion}

This dissertation has considered mainly ideal environments in magnetospheric ULF waveparticle interactions for both short and long time scales, where test-particle simulations and L-shell variance calculations have been used. The only non-ideal case that was brought up here was a pre-storm commencement event on Nov 7, 2004, for which we extracted MHD fields from the OpenGGC model and in which we modeled the diffusion coefficient for a period of 12 hours. We will discuss our findings with both the ideal and the realistic MHD event in parallel below.

The short term wave-particle interactions are adiabatic in that the relation between the kinetic electron energy and the local magnetic field is maintained a constant. As long as the invariant

$\mu$ remains a fixed value the phase space trajectories caused by interaction with a monochromatic ULF wave constructs a well-known island shape with a separatrix between open and closed curves [see e.g. Lichtenberg and Liebermann, or Ukhorskiy and Sitnov, 2012]. However, in reality electrons have a wide range of values in $\mu$ when a ULF wave triggers in the magnetosphere. In fact, even the initial phase determines the particular solution to the ODEs for the phase space trajectories in Eq. (4.2.7) and (4.2.8). Thus simulations with a global initial distribution of electrons, disregarding fixed values for $\mu$, give trajectories that can be highly asymmetric in energy scattering, both in magnitude and timing. The average scattering out of a global population of electrons, given a full range of MLTs and energies, becomes negligible, while the semi-global (only counting MLTs) scattering lasts for only about 2 hours out of an 6 hour simulation. 
The ULF wave has three field components that can potentially cause electron scattering: $\mathrm{E}_{\mathrm{L}}$, $E_{\phi}$ and $B_{z}$. We find that the maximum energy scattering is 3-10 times more effective for $E_{\varphi}$ than $\mathrm{E}_{\mathrm{L}}$ in a magnetic dipole with a realistic dayside compression amplitude - depending on phase and amplitudes. A similar result has been discussed by e.g. Ukhorskiy et al., 2005. When all components are present in an electromagnetic monochromatic wave there are additional nonlinear effects taking place within equations (2.1.14) and (2.1.15). Compared to adding up the separate simulations by $\mathrm{E}_{\phi}$ and $\mathrm{B}_{\mathrm{z}}$ components there is a $10-15 \%$ difference in energy scattering for resonant electrons over a period of 5 hours. We also see a 2-300\% difference in diffusion coefficient in the MHD case of chapter 6, which is for a period of 12 hours. The interaction between self-consistent electromagnetic waves and electrons is not a well understood phenomenon and further research is encouraged in this area. A similar approach in deriving the diffusion coefficient, like Fei et al. (2006) did with an asymmetric magnetic dipole, would be a worthy undertaking.

We also evaluated electron interactions with two coexisting waves with for a set of small frequency separations and phases, where it is confirmed that multi-resonant transport is possible for overlapping resonances in phase space when the Chirikov criterion is met $(K \geq 1)$. The electron energy scattering enhances with decreasing frequency separation, i.e. increasing $\mathrm{K}$, and is also dependent on the phases of the waves. This is a global acceleration phenomenon that is non-negligible. We found that the onset of scattering occurs after about 1 hour simulation time and lasts indefinitely. The average across all energies within the resonance band also has finite values that should not be neglected.

When the physical problem presented in this dissertation extends to longer time scales (days) the process ceases to be adiabatic due to the introduction of stochastic element in the system. 
Stochasticity is defined as either extrinsic - time dependent wave phase randomization; or intrinsic - decorrelations between particle locations and multiple wave phases. For a sufficient degree of stochasticity within arbitrary time intervals, particle trajectories transit into diffusion. It has been established in Fälthammar (1965) that the local power spectral density (PSD) encountered by the particles accounts for the diffusion rate $\left(\mathrm{D}_{\mathrm{LL}}\right)$, with the assumptions that the fields are stationary and ergodic. Our study did not assume ergodicity, which means that the phases of the modes can change dynamically over time. The dynamic phases are due to either random resets of the modes, with a rate of $\mathrm{f}_{\mathrm{r}}$, or due to particle exposure to waves in a magnetospheric region, which can be defined by the drift frequency $f_{d}$ of electrons passing through these MLT sectors.

In addition to particle simulations we calculate variances in L-shell positions directly via integrations of the wave. Tests of this variance method with several different parameters demonstrate that it works well for systems with broadband spectra. It does fail to reproduce $\mathrm{D}_{\mathrm{LL}}$ for narrowband modes since the integrations must assume constant L-shell positions for the particles. This becomes very obvious for the MHD case in chapter 6 where the $\mathrm{D}_{\mathrm{LL}}$ from the two distinct low-frequency modes diminishes due to the lack of phase recurrence. When using realistic wave fields it is still possible to simply average the PSD for each mode over an arbitrary frequency range in order to find an estimation that matches the variance calculations, as demonstrated in figure 6-19.

Returning to ideal cases, we showed, with both methods, that any mode in a broadband spectrum can contribute to the total diffusion rate for a particular drift frequency within the spectral band via dynamic phases. Each mode contributes maximally at a phase reset frequency of $f_{r}=2.63 f_{k}$, where $f_{k}$ is the mode frequency. We experimented with electron 
diffusion due to interaction with wave broadband spectra in MLT sectors and found the phase reset effect being strongest when there is no azimuthal wave vector $\left(\mathrm{m}_{\mathrm{sec}}=0\right)$ within the sector. $\mathrm{D}_{\mathrm{LL}}$ rapidly coheres to the local PSD as the wave number increases and, for example, at $\left|\mathrm{m}_{\mathrm{sec}}\right|=1.00 \pm 0.25$ the effect of phase resets is only $10-30 \%$ as strong. Thus it can be expected that for either small MLT sector widths (i.e. field line resonances) or a broad sector with small wave numbers, which seems to be indicated in the MHD event presented here, the diffusion coefficient can have a maximum at different frequencies than where the strongest local PSD is.

Since phase resets depend on particle drift frequencies when MLT sectors are involved, a consequence is that the radial position also adjusts the $\mathrm{D}_{\mathrm{LL}}$. From the local PSD as the sole contributor to diffusion, where $\mathrm{D}_{\mathrm{LL}} \alpha \mathrm{L}^{6}$ [Schulz and Lanzerotte, 1979], the function becomes $D_{\text {LL }} \propto \mathrm{L}^{5}$ with some variations due to $\mathrm{f}_{\mathrm{d}}$ and MLT sector width. The implication is that diffusion may be slower than thought at higher L-shells, should the wave activity be confined in a well-defined MLT sector.

It should be emphasized that the purpose of this dissertation has been to understand the mechanisms in wave-particle interaction and what effects the relevant parameters have. It has not been attempted to find the actual outcome for realistic scenarios, not even for the MHD case given. The idea is to allow for further improvements of the models here before a realistic comparison can be made. For example, we made the assumption that the wave fields were identical at all L-shells, when they in fact have radial gradients. By looking at the statistics for the wave $\mathrm{E}_{\phi}$ field amplitude and occurrence in for example figure 3-3 it can be expected to be another significant factor for an increasing scattering width with increasing L-shells. In fact, this may lead to a breakdown of diffusive scattering when a large portion of the electron 
population reaches the magnetopause, and has been reported occurring in theoretical studies by Ukhorskiy et al., 2008 and Degeling et al., 2011.

Not only should the understanding in additional parameters be improved upon, but we also propose further stastistical work with the help of the results in this dissertation. Mapping of magnetospheric ULF wave properties, where parameters such as frequencies, phases, polarizations, duration and location, can be helpful to compare with observed particle fluxes in the outer belt. The parameter of particular interest is the phase, since we have shown its importance in both adiabatic 2-wave transport, as well as diffusion. The phase difference between adjacent modes should be compared, as well as any potential phase resets. The case study of Nov 7, 2004 shown in this dissertation hints on a sudden change in phases at the middle of the time interval, for example. 


\section{Appendix A: Test Particle Solver}

The main part of this thesis is centered on the use of a guiding-center ODE solver. The particle tracing is conducted on the two dimensional equatorial plane, centered at geomagnetic coordinates, where only the first adiabatic invariant (2.1.3) is conserved. A three dimensional particle tracing simulation needs far more computational resources than this simplified model, not only in terms of spatial interpolation, but also due to temporal resolution. The north-south bouncing motion associated with the second invariant must be taken into account if such model was used, thus not only the drift velocities must be evaluated but also tracing the acceleration from the Lorentz force. In terms of stochastic dynamics there would be both radial and pitch angle diffusion to consider. In other words, there would be too many time scales for these phenomena to handle simultaneously.

Thus all particles that are used in the simulations in the scope of this thesis have a $90^{\circ}$ pitch angle. It can also be physically justified by two facts; the azimuthal drift velocity does not vary more than $1 / 3$ between a $0^{\circ}$ and $90^{\circ}$ pitch angle [Fälthammar, 1965], [Schulz \& Lanzarotte, 1976] and most wave activity is observed at the magnetic equatorial plane.

The range of perpendicular energies for particles covers from a few hundred kiloelectronvolts up to mega-electronvolts, which is in the relativistic realm. The overall energy content in the radiation belt particle population for this high energy distribution portion is negligible with respect to typical values of wave power, thus a valid approximation is the neglect of wave damping in the code. 
Each particle is assigned initial values of position and perpendicular energy for each simulated scenario. From these values the initial adiabatic invariant is calculated from (2.1.3) before the trajectory is numerically evaluated. As the time steps begin progression a RungeKutta-Fehlberg procedure handles the differential equations for both radial (2.1.14) and azimuthal (2.1.15) drift paths [Fehlberg, 1969]. For any initial value problem of the form

$$
y^{\prime}=f(t, y), y\left(t_{0}\right)=y_{0}
$$

the solution can be found by using coefficients from adjustable time steps:

$$
\begin{aligned}
& \left.k_{1}=\Delta t f\left(t_{i}, y_{i}\right)\right) \\
& \left.k_{2}=\Delta t f\left(t_{i}+\frac{1}{4} \Delta t, y_{i}+\frac{1}{4} k_{1}\right)\right) \\
& \left.k_{3}=\Delta t f\left(t_{i}+\frac{3}{8} \Delta t, y_{i}+\frac{3}{32} k_{1}+\frac{9}{32} k_{2}\right)\right) \\
& \left.k_{4}=\Delta t f\left(t_{i}+\frac{12}{13} \Delta t, y_{i}+\frac{1932}{2197} k_{1}-\frac{7200}{2197} k_{2}+\frac{7296}{2197} k_{3}\right)\right) \\
& \left.k_{5}=\Delta t f\left(t_{i}+\Delta t, y_{i}+\frac{439}{216} k_{1}-8 k_{2}+\frac{3680}{513} k_{3}-\frac{845}{4104} k_{4}\right)\right) \\
& \left.k_{6}=\Delta t f\left(t_{i}+\frac{1}{2} \Delta t, y_{i}-\frac{8}{27} k_{1}+2 k_{2}-\frac{3544}{2565} k_{3}+\frac{1859}{4104} k_{4}-\frac{11}{40} k_{5}\right)\right)
\end{aligned}
$$

Using all coefficients up to $6^{\text {th }}$ order gives the value at the next time step:

$$
y_{i+1,6 t h}=y_{i}+\frac{16}{135} k_{1}+\frac{6656}{12825} k_{3}+\frac{28561}{56430} k_{4}-\frac{1}{5} k_{5}
$$

The preset time step may be adjusted should the accuracy fall below a desired limit. This can be done by comparing the solution from $6^{\text {th }}$ order with the $5^{\text {th }}$. The adjusted value is 


$$
\frac{\Delta t_{\text {new }}}{\Delta t_{\text {old }}}=\left(\frac{\varepsilon \Delta t_{\text {old }}}{2\left|y_{i+1,5 \text { th }}-y_{i+1,6 \text { th }}\right|}\right)^{1 / 4}
$$

where $\epsilon$ is the error.

Once the particles begin their trajectories they encounter electric and magnetic fields which are either determined analytically with the location of the particle or interpolated from a grid of a polar coordinate system. In the latter the fields are found by linear interpolation with the all the spatial neighbors of the location as well as between the field grid time steps. In one special case (see chapter 5-3) the electric field values are also pre-defined as a time series for each particle individually. 


\section{Appendix B: Table of Symbols}

B - magnetic field

$\mathrm{B}_{\mathrm{z} 0}$ - magnetic field dipole moment

$\mathrm{D}_{\mathrm{LL}}$ or $\mathrm{D}_{\mathrm{WW}}$ - Diffusion coefficient (L-shell or energy)

E - electric field

$\mathrm{f}$, or $\omega / 2 \pi-$ frequency

$\mathrm{K}$ - stochasticity parameter (resonant overlap)

L - L-shell

$q$ - particle charge

$\mathrm{R}_{\mathrm{E}}-$ Earth radius

$\mathrm{v}$ - particle drift velocity

W - kinetic energy

$\beta$ - spectral index

$\phi$-phase

$\varphi$ - azimuthal location (or MLT)

$\gamma-$ relativistic correction factor

$\mu-1^{\text {st }}$ adiabatic invariant 
$\tau$ - characteristic time

$\xi$ - normalization factor due spectral index

$\underline{\text { Indices used: }}$

0 - initial parameter for electrons

$1,2,3, \ldots, \mathrm{k}$ - wave index

A, B or wave - particular wave

band - time correlation for $\mathrm{f}_{\mathrm{Nk}}-\mathrm{f}_{1}$

c- compressed component

$\mathrm{d}-\mathrm{drift}$

dip - dipole component

grid - global grid in field models

int - time interval for the modeled MHD field

loc - local component

$\mathrm{r}$ - reset, randomization

sec - sector

sim - simulation 
w-p - wave-particle

$\alpha$ - Diffusion vs. L-shell power law index

$\Delta \mathrm{f}$ - time correlation for $\mathrm{f}_{\mathrm{k}}-\mathrm{f}_{\mathrm{k}-1}$ 


\section{References}

Anderson, J.M., et al. (1990), A Statistical Study of Pc3-5 Pulsations Observed by the AMPTE/CCE Magnetic Reld Experiment 1. Occurrence Distribution.

Baker, D.N., et al. (1987), Highly Relativistic Magnetospheric Electrons: A Role in Coupling to the Middle Atmosphere?, Geophys. Res. Lett., 14, 1027

Baker, D., T. Pulkkinnen, X. Li, et.al. (1998), Coronal mass ejections, magnetic clouds, and relativistic magnetospheric electron events: ISTP, J. Geophys. Res., 103, A8

Barnes, 1983, Hydromagnetic waves, turbulence, and collisionless processes in the interplanetarymedium, in Solar- Terrestrial Physics: Principles and Theoretical Foundations, edited by R.L. Carovillano, and J.M. Forbes, pp. 155-199, $\quad$ D. Reidel Publishing Company, Dordrecht.

Blake, et al., (1992), Injection of electrons and protons with energies of tens of MeV into L 3 on March 24, 1991, Geophys. Res. Lett., 19, 821-824

Bloom, R.M., and H.J. Singer, (1995), Diurnal trends in geomagnetic noise power in the Pc2 through Pc5 bands at low geomagnetic latitude, J. Geophys. Res., 100, 14,943

Brautigam, D. H., and J. Albert, (2000), Radial Diffusion Analysis of Outer Radiation Belt Electrons during the October 9, 1990, Magnetic Storm, J. Geophys. Res., 105, A1

Burlaga L. F. (1969), Directional Discontinuities in the Interplanetary Magnetic Field, Solar Phys., vol 7, 1, 54-71

Chan, A.A., et al., (1989), Nonlinear Interaction of Energetic Ring Current Protons with Magnetospheric Hydromagnetic Waves, Geophys. Res. Letters, 16 1133-1136 
Chen, L., and A. Hasegawa, (1974), A Theory of Long-Period Magnetic Pulsations: 1. Steady State Excitation of Field Line Resonance, J. Geophys. Res., 79, 7, 1024-1032

Chirikov, B.V. (1959), Resonance Processes in Magnetic Traps, Energ. 6: 630 [ or 1960: Plasma Phys. (J.N.E. Pt. C) 1, 253. ]

Chirikov, B.V., (1979), A Universal Instability of Many-Dimensional Oscillator Systems, Phys. Rep. 52: 263

Claudepierre, S. G., et al. (2010), Solar Wind Driving of Magnetospheric ULF Waves: Field Line Resonances Driven by Dynamic Pressure Fluctuations.

Degeling, A. W., et al., (2007), The Effect of ULF Compressional Modes and Field Line Resonances on Relativistic Electron Dynamics, Adv. Space Res., Planet. Space Sci., 55, 731.

Degeling, A. W., R. Rankin, and S. R. Elkington, (2011), Convective and Diffusive ULF Wave Driven Radiation Belt Electron Transport, J. Geophys. Res., 116 (A12)

Dungey, J. W., (1963), Hydromagnetic Waves and the Ionosphere, Proceedings of the International Conference on the Ionosphere, 230

Dungey, J. W., (1965), Effects of Electromagnetic Perturbations on Particles Trapped in the Radiation Belts, Space Science Rev. 4, 199

Elkington, S.R., M.K. Hudson, and A.A. Chan, Acceleration of relativistic electrons via drift-resonant interaction with toroidal-mode Pc-5 ULF oscillations, Geophys. Res. Lett., 26 $(21), 3273$

Elkington, S.R., M.K. Hudson, and A.A. Chan, Resonant acceleration and diffusion of outer zone electrons in an asymmetric geomagnetic field, J. Geophys. Res., 108 (A3), 1116, doi:10.1029/2001JA009202, 2003. 
Elkington, S.R., 2006, A review of ULF interactions with radiation belt electrons, in Magnetospheric ULF Waves: Synthesis and New Directions, edited by K. Takahashi, P.J. Chi, R.E. Denton, and R.L. Lysak, AGU Geophysical Monograph 169, AGU, Washington, D.C.

Elskens, Y. and Escande, D. (2003). Microscopic dynamics of plasmas and chaos. IoP publishing, Bristol, UK.

Fehlberg, E., (1969), Low-order classical Runge-Kutta formulas with step size control and their application to some heat transfer problems. NASA Technical Report 315.

Fei, Y., A. Chan, S. Elkington and M. Wiltberger (2006), Radial diffusion and MHD particle simulations of relativistic electron transport by ULF waves in the September 1998 storm, J. Geophys. Res., VOL. 111, A12209

Friedel, R. H. W., G. D. Reeves, and T. Obara, Relativistic electron dynamics in the inner magnetosphere - a review, J. Atmos. Solar Terr. Phys., 64 (2), 265, 2002.

Fälthammar, C.-G. (1965), Effects of time-dependent electric fields on geomagnetically trapped radiation, J. Geophys. Res., 70, 2503.

Goldstein, J., (2006), Plasmasphere Response: Tutorial and Review of Recent Imaging Results, Space Science Rev., 124

Green and Kivelson, (2001), A tale of two theories: How the adiabatic response and ULF waves affect relativistic electrons, Journal of Geophys. Res., vol 106, no. A11, 25,777-25,791

Greenstadt, E. W., R. L. McPherron, and K. Takahashi, (1981), Solar wind control of daytime, midperoid geomangetic pulsations, in ULF Pulsations in the Magnetosphere, vol. 89, edited by D.J. Southwood, Center for Academic Publications Japan 
Gurnett, D. A., and A. Bhattacharjee, (2005), Introduction to Plasma Physics, Cambridge University Press

Holzworth and Mozer (1979), Direct Evaluation of the Radial Diffusion Coefficient near $L=6$ due to Electric Field Fluctuations, J. Geophys. Res., 84 (A6), 2559, 1

Huang, C.-L., et al., (2010), Modeling Radiation Belt Radial Diffusion in ULF Wave Fields: 2. Estimating Rates of Radial Diffusion using Combined MHD and Particle Codes, J. Geophys. Res., 115, A06216

Hughes, W.J., (1994), Magnetospheric ULF waves: A Tutorial with a Historical Perspective, in Solar Wind Sources of Magnetospheric Ultra-Low-Frequency Waves, Geophysical Monographs, vol. 81, edited by M. Engebretson, K. Takahashi, and M. Scholer, pp. 1-11, AGU, Washington, D.C.

Jacobs, 1970, Geomagnetic Micropulsations, Springer-Verlag, New York Heidelberg Berlin

Jokipii, J. R., and P. J. Coleman, (1968), Cosmic-Ray Diffusion Tensor and its Variation Observed with Mariner 4. J. Geophys. Res. 73, 5495

Kennel, C. F., and H. E. Petsheck, (1966), Limit on Stably Trapped Particle Fluxes, J. Geophys. Res., 71, 1-28

Kepko, L., and H. E. Spence, (2003), Observations of Discrete, Global Magnetospheric Oscillations Directly Driven by Solar Wind Density Variations, J. Geophys. Res., 108(A6), 1257

Kivelson, M., and D. J. Southwood, (1985), Resonant ULF Waves: A New Interpretation, Geophys. Res. Lett., 12, 49-52 
Kivelson, M., and R. Christopher, (1995), Introduction to Space Physics. Cambridge University Press.

Korth, A., and Friedel, R.H.W., (1996), Dynamics of the Near Earth Radiation Environment: Observations over the Whole CRRES Mission, AIP Conference Proceedings 383, 19 (1996); doi: 10.1063/1.51532

Kress, B.T. et al (2007) Global MHD Test Particle Simulations of $>10 \mathrm{MeV}$ Radiation Belt Electrons During Storm Sudden Commencement

Li, X., et al., (1993), Simulation of the Prompt Energization and Transport of Radiation Belt Particles During the March 24, 1991 SSC (MeV electrons at >6RE heavily energized to new belt at 2.5RE)

Lichtenberg, A. J., and M. A. Lieberman, (1992). Regular and Chaotic Dynamics. Springer, Berlin. ISBN 978-0-387-97745-4.

Liu, W., et al. (2009), Electric and magnetic field observations of Pc4 and Pc5 pulsations in the inner magnetosphere: A statistical study, J. Geophys. Res., 114, A12206

Mann, I. R., and A. N. Wright, (1995), Finite Lifetimes of Ideal Poloidal Alfvén Waves, J. Geophys. Res., 100, A12

Mathie, R. A., and I. R. Mann (2000), A correlation between extended intervals of ULF wave power and storm-time geosynchronous relativistic electron flux enhancements, Geophys. Res. Lett., 27, 3261.

Northrop, T.G., (1963), The Adiabatic Motion of Charged Particles, 109 pp., Interscience Publishers, New York 
O’Brien R. McPherron, D. Sornette, G. Reeves, R. Friedel, and H. Singer. (2001), Which magnetic storms produce relativistic electrons at geosynchronous orbit?, J. Geophys. Res., 106, A8

Olson, W. P., (1970), Coordinate Transformations Used in Magnetospheric Physics, McDonnell Douglas Astronautics Company Paper WD1145

Ozeke, L. G., et al., (2012), ULF Wave-Driven Radial Diffusion Simulations of the Outer Radiation Belt, Geophys. Monog. Series, 199

Paulikas, G.A., and Blake, J.B., (1979), Effects on the Solar Wind on Magnetospheric Dynamics: Energetic Electrons at the Synchronous Orbit, in Quantitative Modeling of Magnetospheric Processes, Geophys. Monograph. Amer. Geophys. Union, 21, 180

Perry, K. L., et al. (2006), Incorporating Spectral Characteristics of Pc5 Waves into Three-Dimensional Radiation Belt Modeling and the Diffusion of Relativistic Electrons

Radoski, H. R., (1976), Hydromagnetic Waves: Temporal Development of Coupled Modes, Environmental Res. Paper, 559

Raeder, J., J. Berchem, and M. Ashour-Abdalla, (1998), The geospace environment grand challenge: Results from a global geospace circulation model, JGR, 103, 14787

Reeves, G.D., (1998), Relativistic Electrons and Magnetic Storms: 1992-1995, Geophys. Res. Letters 25, 3265-3268

Reeves, G.D., et al., (2003), Acceleration and loss of relativistic electrons during geomagnetic storms, Geophys. Res. Letters 30, no. 10, 1529

Roederer, J.G. (1970), Dynamics of Geomagnetically Trapped Radiation, Vol. 2, Springer, New York 
Rostoker, G., S. Skone, and D. N. Baker, (1998), On the origin of relativistic electrons in the magnetosphere associated with some geomagnetic storms, Geophys. Res. Lett., 25, 3701

Schulz, M., and L.J. Lanzerotti, (1974), Particle Diffusion in the Radiation Belts, Physics and Chemistry in Space, vol. 7, Springer-Verlag, New York, 1974.

Selesnick, R. S., et al., 1997, A quiescent state of 3 to $8 \mathrm{MeV}$ radiation belt electrons, Geophys. Res. Lett., 24, no. 12, 1343-1346

Sheldon, R. B., et al., (1998), The discovery of trapped energetic electrons in the outer cusp, Geophys. Res. Lett., 25, 1825-1828

Southwood, D. J., (1974), Some features of field line resonances in the magnetosphere. Planetary and Space $\quad$ Science 22 (3): 483

Stephenson, J. A. E., and A. D. M. Walker, (2002), HF Radar Observations of Pc5 ULF Pulsations Driven by the Solar Wind, J. Geophys. Res., 29, 8-1 to 8-4

Turner D. L., et al. (2012), Explaining Sudden Losses of Outer Radiation Belt Electrons During Geomagnetic Storms

Ukhorskiy, et al., 2005, Impact of toroidal ULF waves on the outer radiation belt electrons, J. Geophys. Res., 110, A10202

Ukhorskiy, A. Y., and M. I. Sitnov, (2008), Radial Transport in the Outer Radiation Belt due to Global Magnetospheric Compressions, Journal of Atm. and Solar-Terr. Phys., 70, p1714-1726

Ukhorskiy, A. Y., et al., (2011), The Role of Drift Orbit Bifurcations in Energization and Loss of Electrons in the Outer Radiation Belt, J. Geophys. Res., 116 (A9)

Ukhorskiy A. Y., and M. I. Sitnov, (2012), Dynamics of Radiation Belt Particles, Space Sci. Rev., DOI 10.1007 
Viall, N. M., L. Kepko, and H. E. Spence, (2009), Relative Occurrence Rates and Connection of Discrete Frequency Oscillations in the Solar Wind Density and Dayside Magnetosphere, J. Geophys. Res., 114, A01201

Walt M., 1994, Introduction to Geomagnetically Trapped Radiation, Cambridge University Press

West H.I., 1972, Shadowing of Electron Azimuthal-Drift Motions near the Noon Magnetopause, Nature Physical Science 240, 6-7

Williams, D.J. (1966), A 27-day Periodicity in Outer Zone Trapped Electron Intensities, J. Geophys. Res., 71, 1815

Wygant, J.F., et al., (1994), Large amplitude electric and magnetic field signatures in the inner magnetosphere during injection of $15 \mathrm{MeV}$ electron drift echoes, Geophys. Res. Lett., 21,1739 